Development of an optoffluidic absorption platform for detection of inorganic ions in water 


\section{Development of an optofluidic absorption platform for detection of inorganic ions in water}

\section{Ontwikkeling van een optofluidische absorptieplatform voor detectie van anorganische ionen in water}




\section{Graduation Committee}

Prof. dr. J. L. Herek (Chairman)

Dr. ir. H. L. Offerhaus (Supervisor)

Dr. A. D. Wexler (Co-Supervisor)

Prof. dr. J. G. E. Gardeniers

Prof. dr. J. C. T. Eijkel

Prof. dr. A. M. D. Richardson

Prof. dr. A. Fiore
University of Twente

University of Twente

Wetsus

University of Twente

University of Twente

Lancaster University

Eindhoven University of Technology

The work described in this thesis was performed in the Optical Sciences research group at the MESA+ institute for nanotechnology at the University of Twente and at Wetsus, European Centre of Excellence for Sustainable Water Technology (www.wetsus.nl). Wetsus is funded by the Dutch Ministry of Economic affairs, the European Union Regional Development Fund, the Province of Fryslân, the City of Leeuwarden and the EZ/Kompas program of the "Samenwerkingsverband Noord-Nederland".

Development of an optofluidic absorption platform for detection of inorganic ions in water

ISBN: 9789463233699

Typeset: LTEX

Printed by: Gildeprint

Cover design: Gerrit Willem Steen

Copyright (C) 2019 by Gerrit Willem Steen 


\section{DEVELOPMENT OF AN OPTOFLUIDIC ABSORPTION PLATFORM FOR DETECTION OF INORGANIC IONS IN WATER}

\section{DISSERTATION}

to obtain

the degree of doctor at the University of Twente, on the authority of the rector magnificus,

Prof. dr. T. T. M. Palstra,

on account of the decision of the graduation committee,

to be publicly defended on

Wednesday the $16^{\text {th }}$ of January 2019 at 14:45

by

\section{Gerrit Willem Steen}

born on June $1^{\text {st }}, 1989$

in Den Ham, The Netherlands 
This dissertation has been approved by:

\section{Supervisor}

Dr. ir. H. L. Offerhaus

and

Co-supervisor

Dr. A. D. Wexler 
Pablo Picasso,

To mums, pups, and sistas 



\section{Contents}

1 Introduction 11

$1.1 \quad$ Water . . . . . . . . . . . . . . . . . . 12

1.1.1 A late start - early scientific understanding . . . . . . . . 12

1.1 .2 Anomalous properties . . . . . . . . . . . . . 12

1.1.3 Hydrogen bond . . . . . . . . . . . . . . . . . . . . 13

1.1.4 Remark on pure water . . . . . . . . . . . . . 13

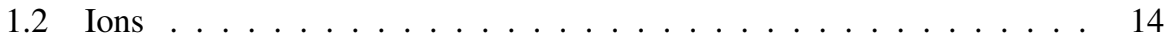

1.2 .1 Ions influence on water . . . . . . . . . . . . . . . . 14

1.2 .2 Hofmeister series . . . . . . . . . . . . . . . . . 15

1.3 Relevance of ionic detection . . . . . . . . . . . . . . 15

1.3 .1 Context . . . . . . . . . . . . . . . . . 15

1.3 .2 Drinking water $\ldots \ldots \ldots \ldots \ldots \ldots \ldots \ldots$

$1.3 .3 \quad$ Industry . . . . . . . . . . . . . . . . . 16

1.4 Method of detection . . . . . . . . . . . . . . . . . . . . . 16

1.4 .1 Optofluidics . . . . . . . . . . . . . . . . . . . . 17

$1.4 .2 \quad$ Spectroscopy . . . . . . . . . . . . . . . . . . 17

1.4 .3 Measuring light . . . . . . . . . . . . . . . . . . 19

1.4.4 Gaussian curves fitting of the $\mathrm{OH}$ vibrational bands . . . . . . 20

1.4.5 Differential absorption . . . . . . . . . . . . . . 20

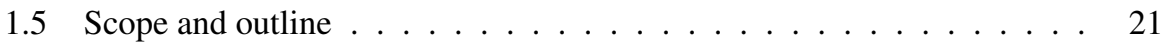

2 Experimental method 31

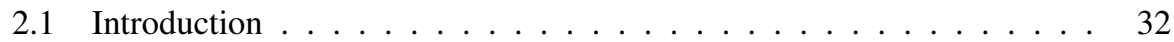

2.1 .1 Fitting strategies $\ldots \ldots \ldots \ldots \ldots \ldots \ldots \ldots \ldots \ldots \ldots$

2.2 Experimental . . . . . . . . . . . . . . . . . . . . 32

$2.2 .1 \quad$ Sample preparation . . . . . . . . . . . . . . 32

2.2.2 Temperature control . . . . . . . . . . . . . . . . . 33

2.2 .3 Measurement conditions . . . . . . . . . . . . . . . 33

2.2.4 Measurement procedure . . . . . . . . . . . . . . . 33

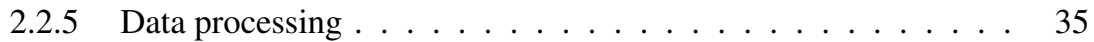

2.3 Results . . . . . . . . . . . . . . . . . . . . . . 37

2.3.1 Frequency-wise linear regression . . . . . . . . . . . . . 37

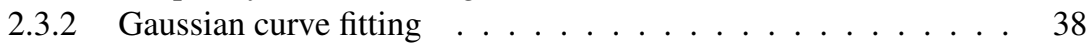

2.4 Discussion . . . . . . . . . . . . . . . . . . . . . . . 40

2.4.1 Frequency-wise linear regression . . . . . . . . . . . . . . . 40

2.4 .2 Gaussian curve fitting . . . . . . . . . . . . . 42

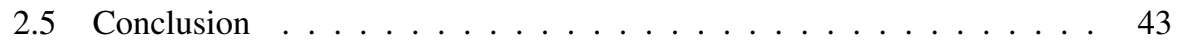


3 Role of temperature 49

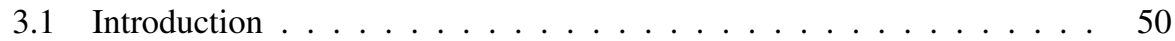

3.2 Methodology . . . . . . . . . . . . . . . . . 51

3.2.1 Sample preparation . . . . . . . . . . . . . . . 51

3.2 .2 Measurement conditions . . . . . . . . . . . . . 51

3.2 .3 Measurements procedure . . . . . . . . . . . . . 52

3.2 .4 Data processing . . . . . . . . . . . . . . . . . . . . 54

3.3 Results \& Discussion . . . . . . . . . . . . . . . . . . . . . . . . . . 59

3.3 .1 Offset temperature effect . . . . . . . . . . . . . . . . 59

3.3.2 Single electrolyte effect $\ldots \ldots \ldots \ldots \ldots$

3.3 .3 Mixture effect. . . . . . . . . . . . . . . . . . 61

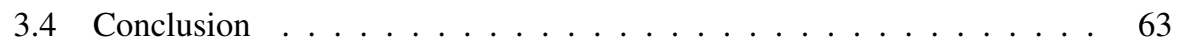

4 Optofluidic chip - designs and fabrication I 69

4.1 Introduction . . . . . . . . . . . . . . . 70

4.2 Design . . . . . . . . . . . . . . . . . . 70

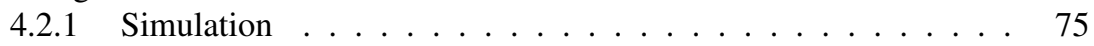

4.3 Tools to investigate optofluidic chips . . . . . . . . . . . . 76

$4.3 .1 \quad$ Optical microscope . . . . . . . . . . . . . . . . . . 78

4.3.2 Scanning electron microscope . . . . . . . . . . . . . . 81

4.3.3 Atomic force microscope . . . . . . . . . . . . . . . . 83

4.4 Lessons learned . . . . . . . . . . . . . . . . . . . . . . . . . . 84

4.5 Optical throughput chips - first run . . . . . . . . . . . . . . 86

4.5.1 Materials \& Procedure . . . . . . . . . . . . . . . . . . 86

4.5 .2 Results . . . . . . . . . . . . . . . . . . . 87

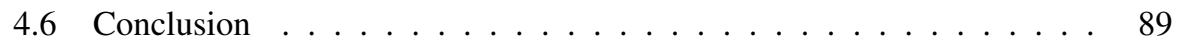

5 Optofluidic chip - designs and fabrication II 95

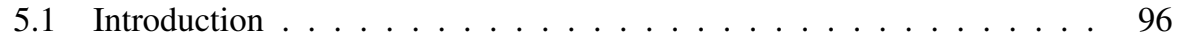

5.2 Cross-section optimization $\ldots \ldots \ldots$. . . . . . . . . . . 97

$5.2 .1 \quad$ Bend losses . . . . . . . . . . . . . . . . . . . . . 100

5.3 Sensing length . . . . . . . . . . . . . . . . . . . 101

5.4 Interferometric detection $\ldots \ldots \ldots \ldots \ldots \ldots$

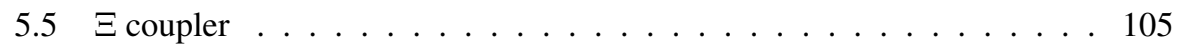

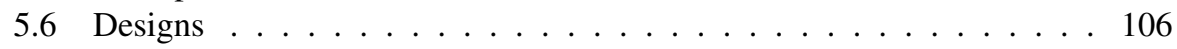

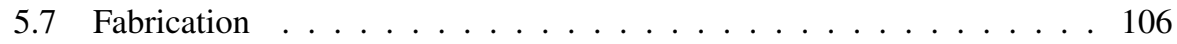

5.8 Optical throughput chips - second fabrication $\ldots \ldots \ldots \ldots \ldots$

5.8 .1 Materials . . . . . . . . . . . . . . . . . . . . 107

5.8 .2 Procedure . . . . . . . . . . . . . . . . . . . 108

5.8 .3 Results . . . . . . . . . . . . . . . . . . . . . . 109

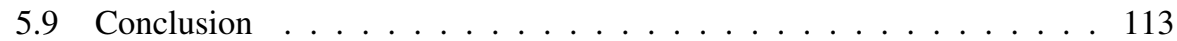

6 Summary and outlook 119

6.1 Reflections . . . . . . . . . . . . . . . . . . 120

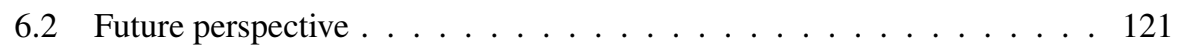

$\begin{array}{lr}\text { A Supplemental Figures - Chapter } 3 & 125\end{array}$ 
B Interferometric data fitting 129

B.1 Broadband Mach Zehnder . . . . . . . . . . . . . . . . . . . . . . . 129

B.2 Implementation . . . . . . . . . . . . . . . . . . . 131

C Microscopic images - first batch optofluidic chips 137

D Mask design - second batch optofluidic chips 145

$\begin{array}{ll}\text { Algemene Nederlandse samenvatting } & 151\end{array}$

$\begin{array}{ll}\text { Acknowledgements } & 153\end{array}$ 


\section{CHAPTER}

\section{Introduction}

66 Life is water, dancing to the tune of macro molecules.

Albert Szent-Gyorgyi, Biochemist 
This thesis describes the development of an integrated optical sensor for the identification and quantification of ionic content in water. The scientific jargon in this chapter is minimized to provide a brief general introduction to the topic and its relevance. First several scientific terms have to be introduced, followed by an explanation of the detection relevance of ionic content in water and finally the methodology is shortly described.

\subsection{Water}

The recent discovery of water traces on Mars led to excitement in the scientific community: The discovery of life on Mars became plausible for water is the key element of life as we know it. [1] More than $70 \%$ in weight and $99 \%$ in number of molecules of our own body consists of water; it is the carrier of nutrients, the regulator of body temperature, it enables removal of unwanted substances; and almost all chemical reactions in life processes occur in aqueous solutions.

\subsubsection{A late start - early scientific understanding}

The ancient Greek philosophers thought water to be one of the four elements: air, fire, earth, and water. It was only in 1766 due to the discovery of hydrogen, the most abundant molecule, that it became apparent that water is actually a molecule. [2] Henry Cavendish claimed water to be composed of two parts hydrogen to one part oxygen which was shortly afterwards confirmed in 1800 by Johan Ritter. [3]

Water is omnipresent; it is the second most abundant molecule in the cosmos and plays an essential role in processes ranging from the nano scale (biological processes) to the macro scale (formation of stars). [4-7] Water might seem a simple molecule: it is odorless, tasteless and at a quick glance transparent in appearance, perhaps this is the reason for the lack of scientific endeavor to study the substance in the late $18^{\text {th }}$ and early $19^{\text {th }}$ century.

\subsubsection{Anomalous properties}

Water is unlike any other liquid. It has more than 70 known anomalies, [8] such as: increased density upon melting, unusually low compressibility, exceptional high heat capacity, decreased viscosity under pressure, it can be extremely slippery and extremely sticky at the same time; and has a high surface tension. Some anomalies of liquid water related with temperature are shown in figure 1.1; the properties deviate from other liquids at ambient temperatures. New anomalies are constantly uncovered, serving well to validate proposed water models. These models try to reveal the underlying principles of the complex ensemble of water molecules in the liquid and solid state. [9]

Single water molecules are thought to be well understood; however water in the fluid (and solid) state with their mutual interactions in different conditions (supercooled, nanoconfined, surface interfaces, etc) remain an ongoing investigation of many different scientific groups specialized in different techniques. Techniques used to probe the structure of water include X-ray diffraction, infrared-, Raman-, ultrafast spectroscopy, 
computational work, neutron scattering, nuclear magnetic resonance, and thermodynamics. Each technique has its size- and time scale of operation; ranging from $10^{-15}$ (femto)seconds to seconds. [10-16]

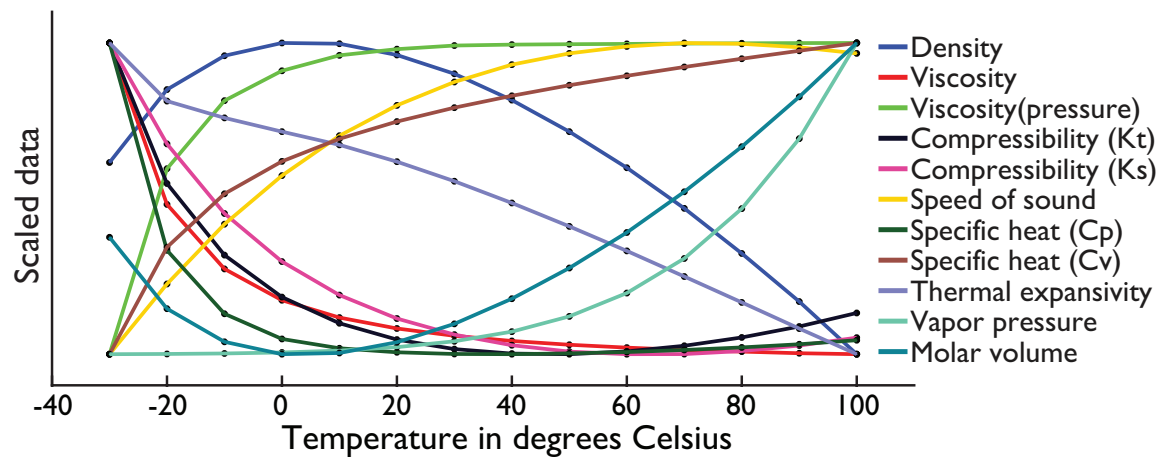

Figure 1.1: Data of several anomalous properties of liquid water in a temperature range from $-30{ }^{\circ} \mathrm{C}$ to $100^{\circ} \mathrm{C}$, scaled with their minimum and maximum values. [17]

\subsubsection{Hydrogen bond}

The first breakthrough to describe the underlying physical mechanisms of the anomalous properties of water was made in 1920: Wendell Latimer and Worth Rodebush suggested that two water molecules can be bound together by the force exerted by the free pair of electrons on one water molecule on a hydrogen held by a pair of electrons onto another water molecule. [18, 19]

This intermolecular bond is now called the hydrogen bond (H-bond). It is weaker than the covalent bond which is formed by sharing of electron pairs between the same or distinct atoms; typically the covalent bond strength is higher than $200 \mathrm{~kJ} \mathrm{~mol}^{-1}$. Also weaker than the ionic bond which is formed due to electrostatic attraction between oppositely charged ions in a chemical compound, usually in a strength range of $17 \mathrm{~kJ} \mathrm{~mol}^{-1}$ to $29 \mathrm{~kJ} \mathrm{~mol}^{-1}$. Yet the $\mathrm{H}$-bond is stronger than a van der Waals interaction which occurs due to difference in electron density on one side of the molecule compare to the other side with a strength of $0.4 \mathrm{~kJ} \mathrm{~mol}^{-1}$ to $4 \mathrm{~kJ} \mathrm{~mol}^{-1}$. [20, 21]

The experimental data collected over the last decades by different techniques to better understand water is enormous and has proven valuable to validate (in)adequacies of proposed water models. The non-uniform interpretations of experimental data lead to different opinions about the dynamics and structure of water. Water is notoriously difficult to model; a uniform model to explain all the anomalous properties of water has yet to be proposed. The difficulties arise due to extensive hydrogen network with its dynamic hydrogen bonding arrangements: the hydrogen bonds in liquid water are reformed at the pico second timescale due to the hindered rotational motions (i.e., librations). [22-30]

\subsubsection{Remark on pure water}

Pure water is a theoretical substance. Compounds dissolve easier in water than in any other fluid which is why water is referred to as the universal solvent. [31] For this reason 
even ultra pure water has contaminations: less than $5.0 \mathrm{ng} \mathrm{g}^{-1}$ total organic carbon, less than $<5$ colony forming units $\mathrm{mL}^{-1}$. It is also likely to be contaminated by tiny dust particles and nanobubbles, though their presence in water of the latter has been a controversial issue. [32] What is meant by pure water in this thesis is water in which the concentration of additional components such as ions, bacteria, viruses is below detection limits ( $<$ parts per billion).
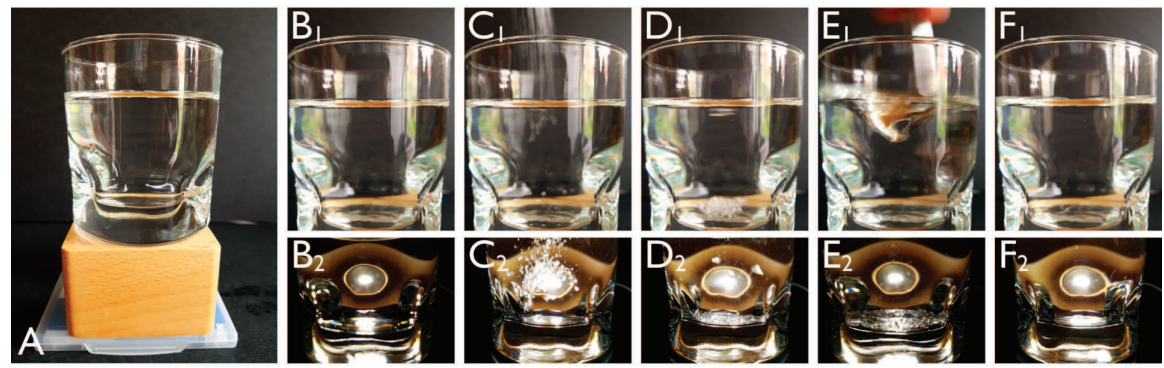

Figure 1.2: Fotographic images of a glass with water. A) A wooden base with a LED is used for illumination. The base is underneath the glass, and at the side of the glass in images with sub indices 1 and 2, respectively. B) Drinking water from the tap. C) Adding kitchen salt. D) The salt grains are visible on the bottom of the glass. E) Stirring the solution with a spoon. F) After stirring.

\section{2 lons}

Ions were discovered by the English chemist Michael Faraday in 1834. He noticed that certain compounds conduct an electric current when dissolved in water. He coined the word ion from ancient Greek " $\iota \omega v o \varsigma$ " meaning "going" to describe the substances that move through the water when an electric potential is applied. Also, he introduced the terms anion and cation for negative and positive charge particles, respectively. [33]

\subsection{1 lons influence on water}

Salt breaks apart into its ionic components: positively and negatively charged particles, when it is dissolved in water. Imagine a transparent holder filled with water and then continue to observe the solution while adding a spoon of kitchen salt $(\mathrm{NaCl})$ as shown in figure 1.2. The stirred solution looks still transparent; the ions do not absorb the visible light (except some metal ions).

Some macroscopic property changes of water can be observed when salt is dissolved; the volume of water, [34] the viscosity (a fluid's resistance to flow), [35] the bubble coalescence, [36] the surface tension (the force responsible for pushing molecules on the surface of a liquid together thereby forming a layer), [37] the conduction, the changes of freezing and boiling point, and the complex refractive index. These changes can be explained by the influences of the ions on the hydrogen bonds. The microscopic details of how ions affect the structure of water is not completely understood. [38, 39] The lack of a standard model of water to describe all its properties makes it even more difficult to understand the mechanisms by which the ions influence their environment. 


\subsubsection{Hofmeister series}

The Hofmeister series originated from the observation of a consistent effect that a series of dissolved salts have on protein solubility wherein anions seem to have a greater impact than cations. [40] Ions are ordered in the Hofmeister series from weakly hydrated cations and strongly hydrated anions to strongly hydrated cations and weakly hydrated anions:

$\mathrm{C}_{6} \mathrm{H}_{5} \mathrm{O}_{7}{ }^{3-}>\mathrm{SO}_{4}{ }^{2-}>\mathrm{HPO}_{4}{ }^{2-}>\mathrm{F}^{-}>\mathrm{Cl}^{-}>\mathrm{Br}^{-}>\mathrm{I}^{-}>\mathrm{NO}^{3-}>\mathrm{CIO}^{4-}$ (anions)

$\mathrm{N}\left(\mathrm{CH}_{3}\right)_{4}{ }^{+}>\mathrm{NH}_{4}{ }^{+}>\mathrm{Cs}^{+}>\mathrm{Rb}^{+}>\mathrm{K}^{+}>\mathrm{Na}^{+}>\mathrm{H}^{+}>\mathrm{Ca}^{2+}>\mathrm{Mg}^{2+}>\mathrm{Al}^{3+}$ (cations) The ions in the Hofmeister series were thought to either to be structure makers or breakers, making or breaking the hydrogen network in water. The weakly hydrated ions are called "chaotropes" (maker) and the strongly hydrated ions are called "kosmotropes" (breakers).

\subsection{Relevance of ionic detection}

\subsubsection{Context}

Wetsus, European centre of excellence for sustainable water technology is a facilitating intermediary for innovating know-how development. It combines innovation goals of companies with knowledge provided by universities to set up Ph.D. programs in different theme groups. This thesis was performed in the theme group "Sensoring" to develop an optofluidic sensor with capabilities to detect ionic content in water. The exact composition of minerals in water is relevant for industry and companies involved with drinking water.

\subsubsection{Drinking water}

A large variety of ions (ionic content) is present in drinking water. Some of the ions are essential and beneficial (e.g., $\mathrm{Cu}^{2+}, \mathrm{Cr}^{3+}$ and $\mathrm{Zn}^{2+}$ ) though toxic in excess, while others are toxic (e.g., $\mathrm{Pb}^{2+}, \mathrm{Hg}^{2+}, \mathrm{Cd}^{2+}, \mathrm{Tl}^{3+}$ ) in trace amounts and bear no known biochemical value. [41]

Our body ceases to operate after water consumption is withheld for approximately three days. [42] The excellent solubility in water of most ionic compounds and an extensive range of organic substances enables an efficient transport of essential nutrients by blood. It is vital to replenish the water in our body without dissolved toxic components; especially those that can build up in our body. Toxicity due to trace contaminants in drinking water can be a slow killer.

The effect of unhealthy water consumption is shown to accumulate over time. For example, 35 to 77 million people in Bangladesh were poisoned with 407 deaths ascertained over a period of nine years due to contamination of ground water with arsenic compounds. [43] Another example of toxic elements are nitrates; it is a known risk factor for infant methemoglobinemia and can progress to cause coma and death if left untreated. $[44,45]$

Guidelines are set by health organizations with the knowledge of the impact of different contaminants on health. Improvements in the accuracy of sensors lead to a better understanding of the impact contaminants have in the environment and for human health. Pharmaceuticals are a concern due to an expected increase of discharges into surface 
water ( $>140$ tons/year pharmaceuticals are discharged into surface waters in the Netherlands alone) and a lack of data concerning quantity and identity of pharmaceuticals in drinking water. $[46,47]$

The concerns about safe drinking water differ per country. Humanitarian aid organizations provide cleaning equipment in $3^{\text {rd }}$ world countries to ensure a better quality of life and educate local population with success: In $201591 \%$ of the global population had access to improved drinking water source as compared to the $76 \%$ in 1990. [48] Still the issue remains that the water may not be safe for consumption due to incorrect equipment handling and timely replacement of equipment. Cheap sensors integrated into cleaning equipment are required for wide spread quality assurance.

Even in developed and wealthy countries water poisoning can occur, due to lack of legal regulations of wastewater and contamination leaks. [49] The distribution system can be contaminated by illegal tapping into the pipes and breaks in pipes. [50-53] Caution for natural poisoning should however not be the only priority; the dangers of bioterrorism have to be considered as well. On-line and real-time sensors can be the first line of defense. [54-56]

Current first identifier of contaminated water is often human illness. Future innovation such as the development of real time and on-/in line detectors for ionic content, bacteria, viruses, and pharmaceuticals could thus improve our quality of life. Data could be collected from a network of smart water pipes (water pipes with sensors) and, in a combination of general health observation in a region, would provide further insight on the impact drinking water has on our health.

\subsubsection{Industry}

Not only the government sector is pushing towards the improvements of sensors; process control in many industrial plants relies on an exact composition of minerals in water to produce products with a certain standard. The common procedure for identification and quantification of different ions in water is sample collection at discrete intervals of time which are then sent to specialists in a dedicated lab. The potential saving of resources motivates the industry to spend time and money on development of innovative sensors. Potential users can be found in the agriculture, biotechnology, food and beverages, electroplating, environmental analysis, metal processing, mining, pharmaceutics, power plant chemistry, pulp and paper, and the semiconductor industry.

\subsection{Method of detection}

Classical techniques for detection of ionic content in water include methods such as titration, gravimetry, turbidimetry, colorimetry and electrochemistry; potentiometry, coulometry or voltammetry. Most of these methods are able to quantify the total amount of ionic concentration but cannot differentiate between ionic species. The standard method for determination of ionic content is ion chromatography (IC). [57-59]

An ideal sensor should, for detection of ionic content, have the following characteristics: 
- (Ia) Capable of identification and (b) quantification of ionic content in water. This requires a specific response due to the different cations and anions in water and their concentration in water.

- (II) Detection limit of sub $\mu \mathrm{M}$ concentration levels.

- (III) Capable of recording sudden changes in ionic content (second time scale).

- (IV) Low production and operation costs. Therefore it should not rely on reagents and a small footprint is required for mass production (reduction of the sensor cost).

- (V) Continuous monitoring, either in the analyte stream (in-line) or in a bypass system (e.g., optofluidic chip).

- (VI) Long operation life time; contamination/clogs are the main causes for reduced operation life time and so these issues should be avoided by design or a self-reliance function.

Many of the classical techniques are transformed to micro scale by means of lab-ona-chip integration. [60, 61] This is the next logical step for sensing: it enables smart devices that are much needed for safety (faster warning system) and industrial purposes (efficient regulations). The sensor platform presented in this thesis operates with an optical method: light is used as a probe to determine which and how many ions are present in a aqueous sample. The necessity to measure in-line requires integration of the optical system; integrated optics means that the light is confined (kept) on a micro / or smaller scale.

\subsubsection{Optofluidics}

For an in-line sensor not only the optics need to be miniaturized, but the fluidics parts as well. Optofluidic is, simply put, the combination of microfluidic and integrated optical systems. The first attempts to create an optofluidic systems were focused on innovative integrated optical devices by incorporation of microfluidic technologies. Since then the field evolved; both the optical and microfluidic platforms gained specific advantages of the other platform. A simple search on scholar.google.com shows (see figure 1.3) articles on optofluidic to be doubled roughly every five years. The research field was recognized in 2003 and has evolved into different direction with a broad range of applications. $[62,63]$

The remote signal and data processing, in-situ/in-line capability, non-intrusive, small and compact are advantages of optofluidic devices that make them suitable candidates for next high technology driven sensor for determination of ionic content in water.

\subsubsection{Spectroscopy}

In 1983 the definition of the meter was resolved by the $17^{\text {th }}$ CGPM to be the length of the path traveled (propagated) by light in vacuum during a time interval of 1/299 792458 of a second. [64] Light is an electromagnetic wave and thus propagates through vacuum with $3 \times 10^{8} \mathrm{~m} \mathrm{~s}^{-1}$. The phase velocity is affected by the interaction with charges like 


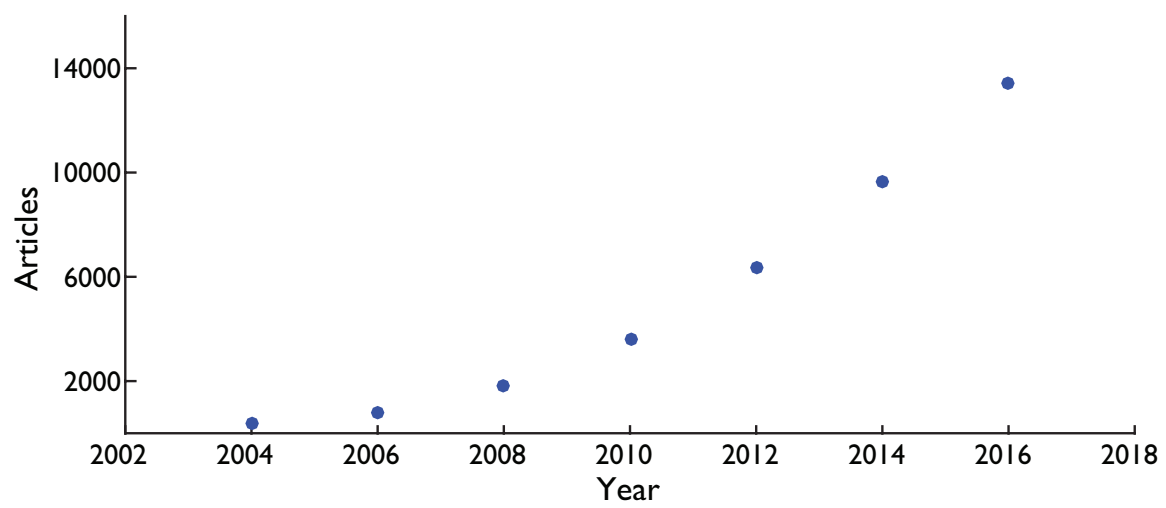

Figure 1.3: Number of publications founds on scholar.google.com with search term 'Optofluidic' over the years since 1990 .

electrons and ion cores. The influence of a medium on an electromagnetic wave by a medium was (presumably) defined by Thomas Young with a dimensionless number: the refractive index $(n)$

$$
n(\lambda)=c / v(\lambda)
$$

Light can be described as either wave or particle. Depending on the phenomena it is either easier to explain the behavior of light using the wave or particle description. Interference of light; e.g., responsible for the different colors of soap bubbles, can be explained by the wave properties of light. The spectral absorption of light can be explained using the particle behavior light; light-matter interaction can be explained with discrete packages of energy of light: photons. The energy of a quanta $(E)$ can be calculated using the Planck constant $(h)$ and the radiation frequency $(f)$ or the speed of light $(c)$ and radiation wavelength $(\lambda)$ :

$$
E=h f=h c / \lambda .
$$

Three processes can occur when light propagates through a medium 1) the light is transmitted 2) the light is scattered, and 3) the light is absorbed (actually a special case of scattering). As a simple example imagine four transparent glasses with water standing on a table while the sun is shining in the background. The sun looks bright blue, dark blue, pale pink, and white by observation of the light source through the different vases after dissolving copper sulfate (low and high amounts), manganese sulfate and sodium nitrate in each vase, respectively. The quantity that can describe the amount of light passing through the vases is the transmission.

Different materials absorb different colors (wavelengths) of light. In the example the iron ions absorb other wavelength than the magnese ions, hence the difference in perceived color, while the concentration of ions lead to different level of transmission. The relations of light loss and length of sample, type of sample and concentration of sample is given by the Beer-Lambert law (1.3). The amount of light of a certain wavelength lost due to interaction with a medium can be quantified with spectral absorption: the linear 
relation between the absorption $(A)$ of light of a certain wavelength $(\lambda)$ by a medium and the length $(L)$, concentration $(c)$ and molar absorptivity $(\epsilon)$, [65]

$$
A(\lambda)=\epsilon(\lambda) c L .
$$

Another dimensionless number is used to describe the influence a medium has on the phase velocity of light as well as the intensity of the light: the complex refractive index $(\tilde{n})$,

$$
\tilde{n}(\lambda)=n(\lambda)+i k(\lambda) .
$$

The extinction coefficient $k=$ is equal to $0.25 \alpha \lambda / \pi$, where $\alpha$ stands for the attenuation coefficient of light. The attenuation coefficient is related to absorbance ( $A b s$ ) by

$$
\alpha=\ln _{10} A b s .
$$

The color of water is perceived to be pale blue when white light is observed through a two meter water filled tube. This hue is due to the higher attenuation of the longer wavelengths (blue light has a shorter wavelength than red light), so of the white light, more blue light is transmitted (see figure 1.4).

\subsubsection{Measuring light}

A spectrometer can be used to record the spectral absorbance of a sample. It consists of a dispersing element to separate the wavelengths of the light source, e.g., a prism, and a detector, usually an array of photo diodes. The photoelectric effect (freeing an electron by a photon) is used in photo diodes to quantify the light intensity. The efficiency in which light can free electrons from a medium is given by the quantum efficiency (QE): $Q E=N_{e} / N_{v}$, where $N_{e}$ is the number of produced electron and $N_{v}$ the amount of produced photons. The absorbance of light due to absorption and scattering can be calculated with recorded intensities of light before $\left(I_{0}\right)$ and after $(I)$ it interaction with a sample

$$
\text { Abs }=\log _{10}\left(I / I_{0}\right) .
$$

The energy of photons used in light - matter interactions determines what type of information can be collected: Electronic transitions can be probed with ultraviolet (UV, $100 \mathrm{~nm}$ to $400 \mathrm{~nm}$ ) absorption spectroscopy, vibrational transitions can be recorded with near-/mid infrared (NIR, $0.7 \mu \mathrm{m}$ to $2.6 \mu \mathrm{m} / \mathrm{IR}, 2.6 \mu \mathrm{m}$ to $25 \mu \mathrm{m}$ ) absorption spectroscopy or by recording the spectral inelastic scattered light (Raman spectroscopy), and rotation and spin transitions can be investigated with far infrared (FIR, $25 \mu \mathrm{m}$ to $200 \mu \mathrm{m}$ ) spectroscopy.

The fundamental resonance frequencies of the bend, symmetric- and asymmetric stretch of the $\mathrm{OH}$ vibration lie in the infrared $\left(v_{2}=6.08 \mu \mathrm{m}, v_{1}=3.05 \mu \mathrm{m}\right.$ and $v_{3}=2.87 \mu \mathrm{m}$, respectively). The $\mathrm{OH}$ bond does not only absorb energy at the fundamental vibration but can also vibrate at higher frequencies. The absorption bands in the near-infrared (NIR) region are due to combinations of the bend and the symmetrical- and asymmetrical stretch overtones of the covalent $\mathrm{OH}$ bonds as indicated in figure 1.4. As can be observed the absorption decreases at higher overtones (shorter wavelengths). 


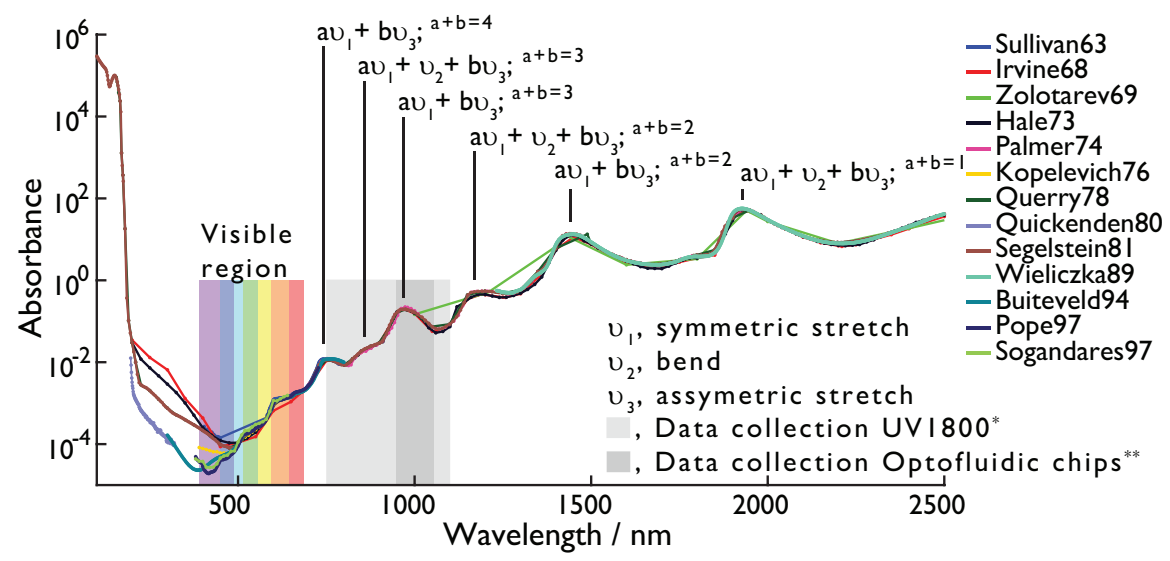

Figure 1.4: Absorbance of light propagating through $1 \mathrm{~cm}$ pure water calculated from absorption coefficient data of several articles. Data collection of the second overtone absorption values of the stretching band were collected from a spectral window of $0.75 \mu \mathrm{m}$ to $1.1 \mu \mathrm{m}\left(^{*}\right)$ for chapters 2 and 3 and $0.94 \mu \mathrm{m}$ to $1.04 \mu \mathrm{m}(* *)$ for chapter 5 and 6 . [66-78]

\subsubsection{Gaussian curves fitting of the $\mathrm{OH}$ vibrational bands}

It is unclear what physical aspect of the hydrogen bonds influence the $\mathrm{OH}$ vibrational absorption bands. It is thought to be either one or more of the following: the type of hydrogen bond, [79] the bending angles of the hydrogen bond, [80] the nature of the formed or broken donor and acceptor hydrogen bonds, [81] the number of hydrogen bonds, [82] and the length of the hydrogen bonds. [83] These different views leads to lack of consensus on the number of underlying absorptions. The absorption bands also reflect coherent energy transfer of several to many water molecules which may lead an inherently flawed analysis when Gaussian curves are fitted on individual water molecules. [84] However, it has been shown experimentally that the absorption spectrum can be fitted using 3, 4 and 5 Gaussian curves. [85]

\subsubsection{Differential absorption}

Ionic identification relying solely on direct light-ion interactions seems unlikely: to identify different ions UV light is required to probe the electronic vibrations. This would lead to an exotic materials choice to enable light propagation on chip and both an expensive light source and detector. Alternatively, the vibrations of the polyatomic ions could be observed with IR light, but yet again the materials, light source and detector would be too expensive and not suitable for detection of mono atomic ions. Another optical method based on the refractive index would be suitable for quantifying ions. However membranes or other separation device are required to identify which ion is quantified.

The optical method used in this thesis observes the influence ions have on the $\mathrm{OH}$ vibrations of water. Kosmotropes cause the absorption peak of the $\mathrm{OH}$ vibrations of water to become broader and induces a bathochromic shift (shift to higher wavelengths, e.g., $\mathrm{F}^{-}$), while chaotropes cause the opposite: narrowing and a hypsochromic shift of this 
peak (shift to lower wavelengths, e.g., $\mathrm{I}^{-}$). It is still debated whether ions have long range influences beyond their valance shells. [86-89]

Water has vibrational absorption overtone bands spreading over a suitable spectral bandwidth for optical integration purposes as shown in figure 1.4. The first overtone bands lay in the telecoms region that can be probe using light sources and detectors make out of Germanium (Ge) or Indium Gallium Arsenide (InGaAs). These materials are more expensive than silicon $(\mathrm{Si})$ based devices, however this spectral region holds the advantage of extensive scientific research. Less familiar is the second overtone band of the $\mathrm{OH}$ stretch; the wavelength regime of $900 \mathrm{~nm}$ to $1050 \mathrm{~nm}$. It can be probed by utilization of cheap silicon sources and detectors, while the required path length on-chip to build up the sensitivity is roughly $10 \mathrm{~cm}$. The third overtone $\mathrm{OH}$ band is impractical due to required path length of $\sim 100 \mathrm{~cm}$.

\subsection{Scope and outline}

Ions perturb the vibrational modes of water (figure 1.4). The fundamental (symmetrical and asymmetrical) vibrations of the $\mathrm{OH}$ stretch lay around $3 \mu \mathrm{m}$. The NIR absorbance bands of water has its origin in overtone $\left(\mathrm{a} v_{1}+\mathrm{b} v_{3}\right)$ bands and combination $\left(\mathrm{a} v_{1}+v_{2}+\mathrm{b} v_{3}\right)$ bands. Two wavelength regions were considered for the development of a sensor for the identifying and quantify electrolytes, the first overtone (second harmonic) around $1500 \mathrm{~nm}$ and the second overtone (third harmonic) of stretching band around $1000 \mathrm{~nm}$. The $1470 \mathrm{~nm}$ window, could take advantage of the knowledge of the telecommunication industry. We choose to develop a platform for absorbance based sensors in the spectral region around $970 \mathrm{~nm}$. Here the advantages are lower propagation losses, cheaper sources and cheaper detectors. This thesis describes the development of a planar waveguide (TriPleX) based integrates optical absorption spectrometry sensor platform for detection of inorganic ionic contaminants in water. The first part of the work describes the methodology and measurements with bulk optics on the second overtone bands of the $\mathrm{OH}$ bond. The second part describes the integration of bulk optics to an optofluidic sensor.

In chapter 2 the basic methodology for single electrolyte identification and quantification is established. Two fitting methods are demonstrated by identification and quantification of sixteen inorganic salts dissolved in water; Gaussian curve fitting and frequency-wise linear regression fitting of Near-Infrared difference absorbance spectra.

Chapter 3 extends on this knowledge by discussing the role of temperature on the sensor response. Three cases are studied: the temperature effect on the absorbance spectrum of demineralized water, the temperature effect on the absorbance spectra of five single electrolytes $\left(\mathrm{NaNO}_{2}, \mathrm{NaNO}_{3}, \mathrm{CaCl}_{2}, \mathrm{~K}_{2} \mathrm{CO}_{3}\right.$, and $\left.\mathrm{NaOH}\right)$ and the temperature effect on the absorbance spectra of two electrolyte mixtures $\left(\mathrm{Na}_{2} \mathrm{SO}_{4} / \mathrm{NaNO}_{3}\right.$ and $\left.\mathrm{Na}_{2} \mathrm{CO}_{3} / \mathrm{NaCl}\right)$

Chapter 4 presents the road-map to integrate the optical methodology to chip level. The first fabricated chip had some issues that are discussed. Different tools are used to investigate the issues and led to redesigning the optofluidic sensors.

Chapter 5 presents the improved design considerations used to fabricate the second batch of optofluidic chips. The results of the simulations are discussed and initial recording of integrated optical differential absorption method is presented.

Finally, chapter 6 holds the concluding remarks and mentions future directions. 



\section{Bibliography}

[1] NASA, JPL, and U. of Arizona, "Nasa confirms evidence that liquid water flows on today's mars." https: / /www.nasa.gov/press-release/nasaconfirms-evidence-that-liquid-water-flows-on-today-smars. Accessed: 12.07.2017. - p.12.

[2] H. Cavendish, "Three papers, containing experiments on factitious air, by the hon. henry cavendish, f. r. s.," Philosophical Transactions, vol. 56, pp. 141-184, 1766. - p.12.

[3] P. R. Roberge, "Johann wilhelm ritter." http://www.corrosiondoctors.org/Biographies/RitterBio.htm. Accessed: 25.07.2017. - p.12.

[4] M. Chaplin, "Water structure and science." http://www1.1sbu.ac.uk/ water/. Accessed: 12.10.2017. - p.12.

[5] M. S. P. Sansom and P. C. Biggin, "Biophysics: Water at the nanoscale," Nature, vol. 414, no. 6860, pp. 156-159, 2001. - p..

[6] N. Smolentsev, W. J. Smit, H. J. Bakker, and S. Roke, "The interfacial structure of water droplets in a hydrophobic liquid," Nature Communications, vol. 8, no. 15548, 2017. - p..

[7] B. Nisini, "Water's role in making stars," Science, vol. 290, no. 5496, pp. 15131514, 2000. - p.12.

[8] M. Chaplin, "Anomalous properties of water." http: / / www1.1sbu.ac.uk/ water/water_anomalies.html. Accessed: 18.09.2017. - p.12.

[9] J. F. Ouyang and R. P. A. Bettens, "Modelling water: A lifetime enigma," CHIMIA International Journal for Chemistry, vol. 69, no. 3, pp. 104-111, 2015. - p.12.

[10] A. K. Soper, "Supercooled water: Continuous trends," Nature materials, vol. 13, no. 7, pp. 671-673, 2014. - p.13.

[11] V. Holten, C. Qiu, E. Guillerm, M. Wilke, J. Rička, M. Frenz, and F. Caupin, "Compressibility anomalies in stretched water and their interplay with density anomalies," ArXiv e-prints, July 2017. - p..

[12] A. W. Omta, M. F. Kropman, S. Woutersen, and H. J. Bakker, "Influence of ions on the hydrogen-bond structure in liquid water," The Journal of Chemical Physics, vol. 119, no. 23, pp. 12457-12461, 2003. - p..

[13] A. Nilsson and L. G. M. Pettersson, "The structural origin of anomalous properties of liquid water," Nature Communications, vol. 6, no. 8998, 2015. — p..

[14] D. Chandler, "Interfaces and the driving force of hydrophobic assembly," Nature, vol. 437, no. 7059, p. 640, 2005. - p..

[15] F. Mallamace, C. Corsaro, D. Mallamace, S. Vasi, and H. E. Stanley, "Nmr spectroscopy study of local correlations in water," The Journal of Chemical Physics, 
vol. 145 , no. 21 , p. $214503,2016 .-$ p..

[16] J. C. Vincent, S. M. Matt, B. M. Rankin, R. D’Auria, J. A. Freites, D. Ben-Amotz, and D. J. Tobias, "Specific ion interactions with aromatic rings in aqueous solutions: Comparison of molecular dynamics simulations with a thermodynamic solute partitioning model and raman spectroscopy," Chemical Physics Letters, vol. 638, pp. $1-8,2015$. - p.13.

[17] M. Chaplin, "Liquid water physical data changes with temperature." Accessed: 15.08.2017. - p.13.

[18] W. M. Latimer and W. H. Rodebush, "Polarity and ionization from the standpoint of the lewis theory of valence.," Journal of the American Chemical Society, vol. 42, no. 7, pp. 1419-1433, 1920. - p.13.

[19] K. Stokely, M. G. Mazza, H. E. Stanley, and G. Franzese, "Effect of hydrogen bond cooperativity on the behavior of water," Proceedings of the National Academy of Sciences, vol. 107, no. 4, pp. 1301-1306, 2010. — p.13.

[20] J. Teixeira, A. Luzar, and S. Longeville, "Dynamics of hydrogen bonds: how to probe their role in the unusual properties of liquid water," Journal of Physics: Condensed Matter, vol. 18, no. 36, p. S2353, 2006. - p.13.

[21] C. J. Fecko, J. D. Eaves, J. J. Loparo, A. Tokmakoff, and P. L. Geissler, "Ultrafast hydrogen-bond dynamics in the infrared spectroscopy of water," Science, vol. 301, no. 5640, pp. 1698-1702, 2003. - p.13.

[22] I. Ohmine and H. Tanaka, "Fluctuation, relaxations, and hydration in liquid water. hydrogen-bond rearrangement dynamics," Chemical Reviews, vol. 93, no. 7, pp. 2545-2566, 1993. - p.13.

[23] H. Martiniano and N. Galamba, "Insights on hydrogen-bond lifetimes in liquid and supercooled water," The Journal of Physical Chemistry B, vol. 117, no. 50, pp. 16188-16195, 2013. - p..

[24] F. N. Keutsch and R. J. Saykally, "Water clusters: Untangling the mysteries of the liquid, one molecule at a time," Proceedings of the National Academy of Sciences, vol. 98, no. 19, pp. 10533-10540, 2001. - p..

[25] F. N. Keutsch and R. J. Saykally, "Water clusters: untangling the mysteries of the liquid, one molecule at a time," Proceedings of the National Academy of Sciences, vol. 98, no. 19, pp. 10533-10540, 2001. - p..

[26] S.-H. Chen, J. Teixeira, and R. Nicklow, "Incoherent quasielastic neutron scattering from water in supercooled regime," Physical Review A, vol. 26, no. 6, p. 3477, 1982. - p..

[27] J. Teixeira, M.-C. Bellissent-Funel, S.-H. Chen, and A.-J. Dianoux, "Experimental determination of the nature of diffusive motions of water molecules at low temperatures," Physical Review A, vol. 31, no. 3, p. 1913, 1985. - p..

[28] I. Brovchenko and A. Oleinikova, "Multiple phases of liquid water," ChemPhysChem, vol. 9, no. 18, pp. 2660-2675, 2008. - p..

[29] L. G. M. Pettersson, R. H. Henchman, and A. Nilsson, "Water - the most anomalous liquid," 2016. - p..

[30] A. Luzar and D. Chandler, "Hydrogen-bond kinetics in liquid water," Nature, vol. 379 , no. 6560 , p. 55, 1996 . - p.13.

[31] H. Perlman, "Water, the universal solvent." http: / / water.usgs.gov/edu/ solvent.html. Accessed: 12.07.2017. - p.13. 
[32] T. Temesgen, T. T. Bui, M. Han, T. il Kim, and H. Park, "Micro and nanobubble technologies as a new horizon for water-treatment techniques: A review," Advances in Colloid and Interface Science, vol. 246, no. Supplement C, pp. 40 - 51, 2017. - p.14.

[33] M. Faraday and J. Tyndall, "Experimental researches in electricity." https: / / archive.org/details/experimentalrese00faraiala. Accessed: 03.08.2017. - p.14.

[34] G. P. Baxter, A. C. Boylston, E. Mueller, N. H. Black, and P. B. Goode, "The refractive power of the halogen salts of lithium, sodium and potassium in aqueous solution.," Journal of the American Chemical Society, vol. 33, no. 6, pp. 901-922, 1911. - p.14.

[35] D. J. P. Out and J. M. Los, "Viscosity of aqueous solutions of univalent electrolytes from 5 to $95^{\circ}$ c," Journal of Solution Chemistry, vol. 9, pp. 19-35, Jan 1980. p.14.

[36] V. S. J. Craig, B. W. Ninham, and R. M. Pashley, "Effect of electrolytes on bubble coalescence," Nature, vol. 364, no. 6435, pp. 317-319, 1993. - p.14.

[37] L. Onsager and N. N. T. Samaras, "The surface tension of debye-hückel electrolytes," The Journal of Chemical Physics, vol. 2, no. 8, pp. 528-536, 1934. — p.14.

[38] E. Brini, C. J. Fennell, M. Fernandez-Serra, B. Hribar-Lee, M. Luksic, and K. A. Dill, "How water's properties are encoded in its molecular structure and energies," Chemical reviews, vol. 117, no. 19, pp. 12385-12414, 2017. — p.14.

[39] J. M. Rodgers and T. Ichiye, "Multipole moments of water molecules and the aqueous solvation of monovalent ions," Journal of Molecular Liquids, vol. 228, pp. $54-62,2017$. From simple liquids to macromolecular solutions: recent experimental and theoretical developments. In Honor of the 70th birthday of Vojko Vlachy. - p.14.

[40] F. Hofmeister, "Zur lehre von der wirkung der salze," Archiv für experimentelle Pathologie und Pharmakologie, vol. 24, pp. 247-260, Feb 1888. - p.15.

[41] J. Plant, Pollutants, human health, and the environment : a risk based approach. Chichester, West Sussex, U.K. Hoboken, N.J: Wiley-Blackwell, 2012. — p.15.

[42] B. M. Popkin, K. E. D'Anci, and I. H. Rosenberg, "Water, hydration, and health," Nutrition Reviews, vol. 68, no. 8, pp. 439-458, 2010. — p.15.

[43] M. Argos, T. Kalra, P. J. Rathouz, Y. Chen, B. Pierce, F. Parvez, T. Islam, A. Ahmed, M. Rakibuz-Zaman, R. Hasan, G. Sarwar, V. Slavkovich, A. van Geen, J. Graziano, and H. Ahsan, "Arsenic exposure from drinking water, and all-cause and chronicdisease mortalities in bangladesh (heals): a prospective cohort study," The Lancet, vol. 376, no. 9737, pp. 252-258, 2010. - p.15.

[44] L. Knobeloch, B. Salna, A. Hogan, J. Postle, and H. Anderson, "Blue babies and nitrate-contaminated well water," Environ Health Perspect, vol. 108, no. 7, pp. 675-678, 2000. - p.15.

[45] M. Kumar and A. Puri, "A review of permissible limits of drinking water," Indian J Occup Environ Med, vol. 16, no. 1, pp. 40-44, 2012. - p.15.

[46] W. D. W. Q. Committee, "Water Sampling and Analysis," Guidelines for drinking water quality, vol. 4th ed., pp. 51-72, 2011. - p.16.

[47] C. T. A. Moermond, C. E. Smit, R. C. van Leerdam, N. G. F. M. van der Aa, and M. H. M. M. Montforts, "Geneesmiddelen en waterkwaliteit," RIVM Briefrapport 
2016-0111, 2016. - p.16.

[48] UNICEF and WHO, Progress on drinking water and sanitation: 2015 Update and MDG Assessment. New York, N.Y. Geneva: UNICEF World Health Organization, 2012. - p.16.

[49] AD (online newspaper), "Ook genx aangetroffen in alblasserdams leidingwater." http: / / www.alblasserdamsnieuws.nl/wordpress/2017/04/21/ ook-genx-aangetroffen-in-alblasserdams-leidingwaterd66-wil-landelijke-aandacht. Accessed: 12.07.2017. — p.16.

[50] AD (online newspaper), "Bron van drinkwater in deel randstad vervuild met gif." http: / /www.ad.nl/nieuws/bron-van-drinkwater-in-deelrandstad-vervuild-met-gifa3350191/. Accessed: 12.07.2017. — p.16.

[51] J. Huisman, “Ziektegevallen na verontreiniging van drinkwater met fenol ; een engelse ervaring," $H$ twee $O$ : tijdschrift voor watervoorziening en afvalwaterbehandeling, vol. 18 , no. 20, p. 433, 1985. - p..

[52] I. of Medicine, Global Environmental Health: Research Gaps and Barriers for Providing Sustainable Water, Sanitation, and Hygiene Services: Workshop Summary. Washington, DC: The National Academies Press, 2009. — p..

[53] C. on Public Water Supply Distribution, Drinking water distribution systems : assessing and reducing risks. Washington, D.C: National Academies Press, 2006. - p.16.

[54] G. S. Parnell, C. M. Smith, and F. I. Moxley, "Intelligent adversary risk analysis: A bioterrorism risk management model," Risk Analysis, vol. 30, pp. 32-48, 2010. - p.16.

[55] S. Clarke, "Bacteria as potential tools in bioterrorism, with an emphasis on bacterial toxins," British Journal of Biomedical Science, vol. 62, no. 1, pp. 40-46, 2005. - p..

[56] P. H. Gleick, "Water and terrorism," Water Policy, vol. 8, pp. 481-503, 2006. - p.16.

[57] L. Pujol, D. Evrard, K. Groenen-Serrano, M. Freyssinier, A. Ruffien-Cizsak, and P. Gros, "Electrochemical sensors and devices for heavy metals assay in water: the french groups' contribution," Frontiers in Chemistry, vol. 2, p. 19, 2014. - p.16.

[58] M. E. Bodini and D. T. Sawyer, "Voltammetric determination of nitrate ion at parts-per-billion levels," Analytical Chemistry, vol. 49, no. 3, pp. 485-489, 1977. PMID: 842857 . - p..

[59] B. Bansod, T. Kumar, R. Thakur, S. Rana, and I. Singh, "A review on various electrochemical techniques for heavy metal ions detection with different sensing platforms," Biosensors and Bioelectronics, vol. 94, pp. 443-455, 2017. - p.16.

[60] J. P. Murrihy, M. C. Breadmore, A. Tan, M. McEnery, J. Alderman, C. O’Mathuna, A. P. O'Neill, P. O'Brien, N. Advoldvic, P. R. Haddad, and J. D. Glennon, "Ion chromatography on-chip," Journal of Chromatography A, vol. 924, no. 1, pp. 233 238, 2001. 14th International Symposium on Microscale Separations and Analysis. - p.17.

[61] Y. Dai and C. C. Liu, "A simple, cost-effective sensor for detecting lead ions in water using under-potential deposited bismuth sub-layer with differential pulse voltammetry (dpv)," Sensors, vol. 17, no. 5, p. 950, 2017. — p.17. 
[62] Y. Fainman, Optofluidics: fundamentals, devices, and applications. New York: McGraw-Hill, 2010. - p.17.

[63] S. Pissadakis, Optofluidics, sensors and actuators in microstructured optical fibers. Cambridge, UK: Woodhead Publishing is an imprint of Elsevier, 2015. - p.17.

[64] BIPM, "Resolution 1 of the 17th cgpm (1983)." http: / / www.bipm.org/en/ CGPM/db/17/1/. Accessed: 03.08.2017. - p.17.

[65] A. Beer, "Bestimmung der absorption des rothen lichts in farbigen flüssigkeiten," Annalen der Physik, vol. 162, no. 5, pp. 78-88, 1852. - p.19.

[66] S. Jacques and S. Prahl, "Optical absorption of water compendium." http: / / omlc.org/spectra/water/abs/index.html. Accessed: 22.08.2017. — p.20.

[67] S. A. Sullivan, "Experimental study of the absorption in distilled water, artificial sea water, and heavy water in the visible region of the spectrum," Opt. Soc. Am. J., vol. 53, pp. 962-968, 1963. - p..

[68] W. M. Irvine and J. B. Pollack, "Infrared optical properties of water and ice spheres," Icarus, vol. 8, pp. 324-360, 1968. - p..

[69] V. M. Zolotarev, B. A. Mikhilov, L. L. Alperovich, and S. I. Popov, "Dispersion and absorption of liquid water in the infrared and radio regions of the spectrum," Optics and Spectroscopy, vol. 27, pp. 430-432, 1969. - p..

[70] G. M. Hale and M. R. Querry, "Optical constants of water in the $200 \mathrm{~nm}$ to $200 \mu \mathrm{m}$ wavelength region.," Appl. Opt., vol. 12, pp. 555-563, 1973. — p..

[71] K. F. Palmer and D. Williams, "Optical properties of water in the near infrared," J. Opt. Soc. Am., vol. 64, pp. 1107-1110, 1974. - p..

[72] O. V. Kopelevich, "Optical properties of pure water in the $250-600 \mathrm{~nm}$ range," Opt. Spectrosc., vol. 41, pp. 391-392, 1976. - p..

[73] M. R. Querry, P. G. Cary, and R. C. Waring, "Split-pulse laser method for measuring attenuation coefficients of transparent liquids: application to deionized filtered water in the visible region," Appl. Opt., vol. 17, pp. 3587-3592, 1978. — p..

[74] T. I. Quickenden and J. A. Irvin, "The ultraviolet absorption spectrum of liquid water," J. Chem Phys., vol. 72, pp. 4416-4428, 1980. - p..

[75] D. J. Segelstein, "The complex refractive index of water.," 1981. - p..

[76] D. M. Wieliczka, S. Weng, and M. R. Querry, "Wedge shaped cell for highly absorbent liquids: infrared optical constants of water.," Appl. Opt., vol. 28, pp. 1714-1719, 1989. - p..

[77] H. Buiteveld, J. M. H. Hakvoort, and M. Donze, "The optical properties of pure water," in SPIE Proceedings on Ocean Optics XII (J. S. Jaffe, ed.), vol. 2258, pp. 174-183, 1994. - p..

[78] R. M. Pope and E. S. Fry, "Absorption spectrum (380-700 nm) of pure water. II. integrating cavity measurements," Appl. Opt., vol. 36, pp. 8710-8723, 1997. - p.20.

[79] N. Chumaevskii and M. Rodnikova, "Some peculiarities of liquid water structure," Journal of molecular liquids, vol. 106, no. 2-3, pp. 167-177, 2003. - p.20.

[80] R. Rey, K. B. Møller, and J. T. Hynes, "Hydrogen bond dynamics in water and ultrafast infrared spectroscopy," The Journal of Physical Chemistry A, vol. 106, no. 50, pp. 11993-11996, 2002. - p.20.

[81] A. Millo, Y. Raichlin, and A. Katzir, "Mid-infrared fiber-optic attenuated total 
reflection spectroscopy of the solid-liquid phase transition of water," Applied spectroscopy, vol. 59, no. 4, pp. 460-466, 2005. - p.20.

[82] G. Walrafen, "Raman and infrared spectral investigations of water structure," in The physics and physical chemistry of water, pp. 151-214, Springer, 1972. - p.20.

[83] D. A. Schmidt and K. Miki, "Structural correlations in liquid water: A new interpretation of ir spectroscopy," The Journal of Physical Chemistry A, vol. 111, no. 40, pp. 10119-10122, 2007. - p.20.

[84] M. Yang and J. Skinner, "Signatures of coherent vibrational energy transfer in ir and raman line shapes for liquid water," Physical Chemistry Chemical Physics, vol. 12, no. 4, pp. 982-991, 2010. - p.20.

[85] M. Chaplin, "Vibrational spectra." http://www1.lsbu.ac.uk/water/ water_methods.html. Accessed: 18.09.2017. — p.20.

[86] A. Nilsson and L. G. Pettersson, "The structural origin of anomalous properties of liquid water," Nature communications, vol. 6, p. 8998, 2015. - p.21.

[87] Y. Chen, H. I. Okur, N. Gomopoulos, C. Macias-Romero, P. S. Cremer, P. B. Petersen, G. Tocci, D. M. Wilkins, C. Liang, M. Ceriotti, et al., "Electrolytes induce long-range orientational order and free energy changes in the h-bond network of bulk water," Science advances, vol. 2, no. 4, p. e1501891, 2016. — p..

[88] A. P. Gaiduk and G. Galli, "Local and global effects of dissolved sodium chloride on the structure of water," The Journal of Physical Chemistry Letters, vol. 8, no. 7, pp. 1496-1502, 2017. - p..

[89] A. W. Omta, M. F. Kropman, S. Woutersen, and H. J. Bakker, "Influence of ions on the hydrogen-bond structure in liquid water," The Journal of chemical physics, vol. 119, no. 23, pp. 12457-12461, 2003. - p.21. 

al 


\section{CHAPTER \\ Experimental method}

66 Data do not give up their secrets easily. They must be tortured to confess.

Jeff Hopper, Bell Labs

This chapter has been published as: G. Steen, E. C. Fuchs, A. D. Wexler, H. L. Offerhaus. "Identification and quantification of 16 inorganic ions in water by Gaussian fitting of Near-Infrared $\left(10754-9618 \mathrm{~cm}^{-1}\right)$ difference absorbance spectra". Applied Optics. 2015. 54(19): 5937 - 5942. 


\subsection{Introduction}

Aqueous electrolytes can be identified and quantified with near-infrared (NIR) light as was shown many decades ago [1,2] and remains an interest. [3-5] Spectral changes induced by dissolving salts are used to identify and quantify these electrolytes.

Most of the spectral changes are due to hydrogen bonds. Next to that, one must consider a number of parameters which influence the energy and thus optical activity of a given aqueous solution. Solutes as well as physical perturbation [6] have been shown to influence the number, [7] length, [8] bending angle [9] and type [10] (single, bifurcated or trifurcated) of the hydrogen bond and thus influence the shape, position and amplitude of the spectral features in a given solution.

The physical origin of the absorbance of water in the NIR spectral region remains an area of continued research; however the focus in this manuscript is on the practical side, it does not aim to provide new insight into the complex interpretation of the hydrogen bonds, but rather aims at a measurement and data processing protocol that is applicable to an optical chip-based rapid analytical sensor.

The wavelength range used in this work can be detected by (cheap) silicon detectors and the optical paths of the system are sufficient small to be integrated on a chip e.g. using Triplex technology.

\subsubsection{Fitting strategies}

It is common to use principal component analysis (PCA) techniques to analyze the complex induced spectral changes or their first derivative. [11] Although very useful to demonstrate analytical potential, a PCA analysis does not translate to a measurement strategy or data analysis of single measurements in an obvious way. An overview of the feasibility of established NIR spectroscopy was published recently. [12]

This study uses two different fitting strategies to identify and quantify 16 aqueous electrolytes: Linear fitting and Gaussian curve fitting. The linear fitting results help to derive suitable models with isosbestic points. These points can be used for identification of the electrolytes and the strength of differential absorbance allows their quantification. From the Gaussian curve fitting applied in this work a truth table can be created to identify and quantify electrolytes with a sensor. The spectral data of 16 different electrolytes are presented and two novel fitting strategies applicable to potentially powerful optical sensors are exploited with a spectral bandwidth narrow enough for a single light source.

\subsection{Experimental}

\subsubsection{Sample preparation}

Stock solutions of 16 electrolytes (see table 2.1) of $1000 \mathrm{mM}$ were prepared using demineralized water, precision $( \pm 0.1 \mathrm{mg})$ balance (PIONEER ${ }^{\mathrm{TM}}$, Ohaus, Florham Park, NJ, USA) and reagent or higher grade salts (Sigma Aldrich, St Louis, MO, USA) without further purification. 
Table 2.1: List of the 16 electrolytes investigated

\begin{tabular}{llll}
\hline $\mathrm{Na}_{2} \mathrm{SO}_{4}$ & $\mathrm{Na}_{2} \mathrm{HPO}_{4}$ & $\mathrm{KBr}$ & $\mathrm{KNO}_{3}$ \\
$\mathrm{~K}_{2} \mathrm{CO}_{3}$ & $\mathrm{KF}$ & $\mathrm{KOH}$ & $\mathrm{NaNO}_{3}$ \\
$\mathrm{Na}_{2} \mathrm{SO}_{3}$ & $\mathrm{NaF}$ & $\mathrm{LiOH}$ & $\left.\mathrm{Pb}_{2} \mathrm{NO}_{3}\right)_{2}$ \\
$\mathrm{NaHCO}_{3}$ & $\mathrm{CaCl}_{2}$ & $\mathrm{NaNO}_{2}$ & $\mathrm{NaClO}_{3}$
\end{tabular}

\subsubsection{Temperature control}

The temperature of each cuvette was regulated by two home-built aluminum ovens. Each oven consists of a milled aluminum block, six resistors $5 \Omega, 5 \%, 10 \mathrm{~W}$ and three temperature transducers (AD592CNZ, Analog Devices, Norwood, MA, USA) each with an accuracy of $\pm 0.3 \mathrm{~K}$.

The current through the resistors of each oven was provided by a power supply (ES030-5, Delta Elektronika, Schouwen-Duiveland, ZL, NLD) and controlled by a PID controller (E5EN-H, Omron Corporation, Shiokoji Horikawa, Kyoto, JPN).

Taking this accuracy into account a typical temperature offset error between sample and reference oven of $\pm 0.24 \mathrm{~K}$ and a stability of $\pm 0.01 \mathrm{~K}$ are to be expected.

\subsubsection{Measurement conditions}

Solutions were measured in cuvettes (111-QS, Hellma GmbH \& Co. KG, Müllheim, BW, GER) of $1 \mathrm{~cm}$ absorption. Absorbance spectra were recorded using a transmission based spectrograph (UV1800, Shimadzu, Nishinokyo-Kuwabara-cho, Kyoto, JPN) with a spectral resolution of $1 \mathrm{~nm}$. The difference absorbance spectra were collected with a $0.5 \mathrm{~nm}$ data point resolution from $20000 \mathrm{~cm}^{-1}$ to $9091 \mathrm{~cm}^{-1}(500 \mathrm{~nm}$ to $1100 \mathrm{~nm})$. Absorbance is defined as the negative $\log _{10}$ of the transmission through $1 \mathrm{~cm}$ of liquid and therefore abbreviated as "Abs $\mathrm{cm}^{-1}$ ".

According to manufacturer specifications the root mean squared (RMS) noise level at $14286 \mathrm{~cm}^{-1}(700 \mathrm{~nm})$ is specified to be less than $0.05 \times 10^{-3}$. [13] This value needs to be accounted for twice due to the differential nature of recording.

The temperature of the light source stabilized during one hour of operation. The entire measurement series of the five concentrations of aqueous electrolytes were therefore collected at least one hour after turning on the spectrograph in order to minimize drift due to variation in light source output.

Baseline spectra were recorded with the sample and reference cuvettes filled with $3 \mathrm{~mL}$ of demineralized water and placed inside the ovens set to $(298.15 \pm 0.30) \mathrm{K}$. The cuvettes remained in place throughout the entire measurement series to reduce positioning errors. After recording the entire measurement series of the five concentrations of an aqueous electrolyte, another five baseline spectra were recorded.

\subsubsection{Measurement procedure}

Five concentrations of aqueous electrolytes were prepared by serial dilution: $500 \mathrm{mM}$, $250 \mathrm{mM}, 125 \mathrm{mM}, 62.5 \mathrm{mM}$ and $31.25 \mathrm{mM}$. The first concentration was mixed directly in the cuvette by replacing half of the demineralized water in the sample with $1.5 \mathrm{~mL}$ of 
A
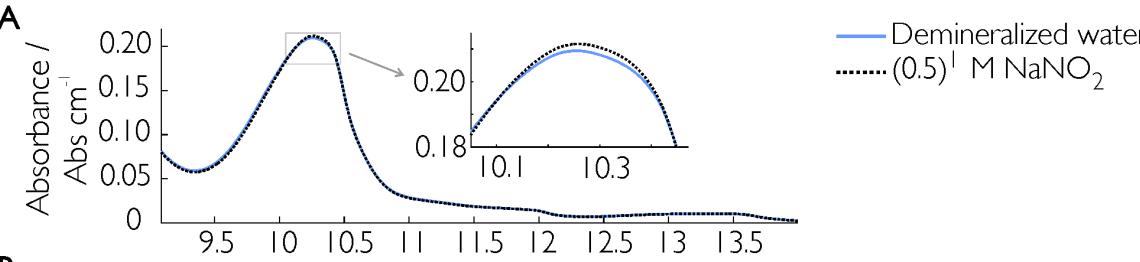

B

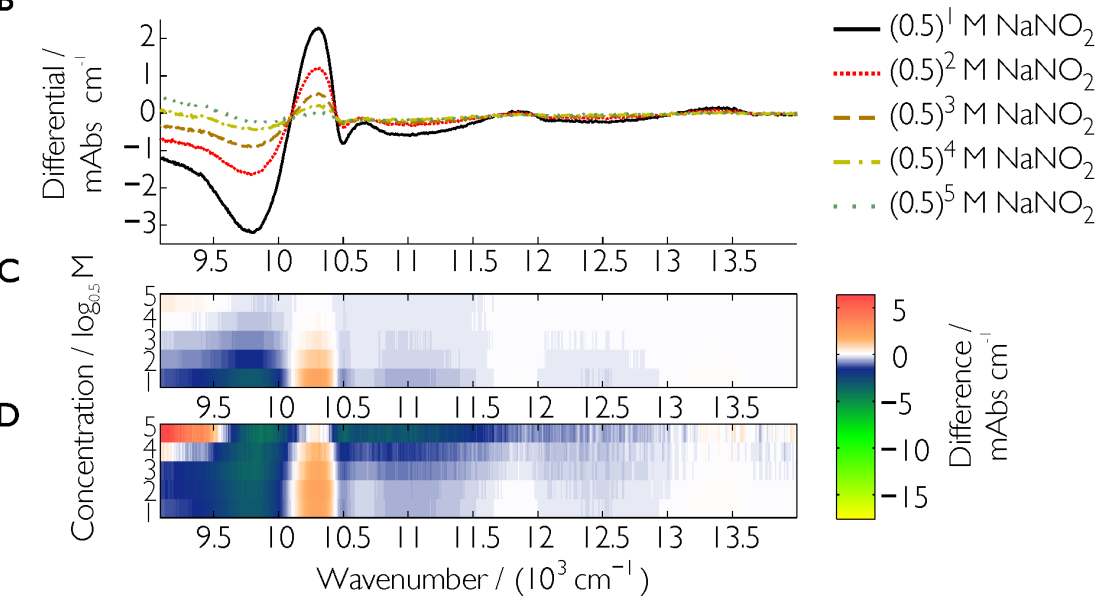

Figure 2.1: (A) Absorbance spectrum of demineralized water (blue-circle) and $(0.5)^{1} \mathrm{M} \mathrm{NaNO}_{2}$ dissolved in demineralized water (black-square). (B) Difference absorbance spectra of demineralized water and aqueous $\mathrm{NaNO}_{2}$, showing the effect of the dissolved salt at different concentration. (C) A different representation of the effect of $(0.5)^{1} \mathrm{M},(0.5)^{2} \mathrm{M},(0.5)^{3} \mathrm{M},(0.5)^{4} \mathrm{M}$ and $(0.5)^{5}$ $\mathrm{M}$ dissolved salt: Five 2D plots are stacked on top of each other with color coded absorbance difference. (D) Multiplication of the difference absorbance spectra shown in (C) with the dilution factor of the electrolyte shows a deviation from the expected Beer-Lambert law, concentrations given in powers of $(0.5)$.

$1000 \mathrm{mM}$ stock solution, using a calibrated pipette (P1000, Gilson, Middleton, WI, USA). Once the sample had reached the set-point temperature $((298.15 \pm 0.30) \mathrm{K}$, typically $2-5$ minutes) five spectra were recorded.

The subsequent concentrations were prepared in the same manner as the first whereby half of the previous dilution was replaced with demineralized water, thermally equilibrated, and then measured. Such a dilution series can be represented mathematically by an inverse power law as $c=d-n$ where $c$ is the molar concentration, $d$ is the base dilution factor (in this work $d=2$ ), and $n$ is the iteration number. The concentrations of the solutions measured in this work can thus easily be written as a series of $(0.5)^{n} \mathrm{~mol} \mathrm{~L}^{-1}$ (or M). Such a power series is especially convenient when using the Beer-Lambert law as the exponent provides the dilution correction factor, $d(n-1)$. [14]

In an additional series of experiments, the influence of temperature difference between sample and reference fluid on the spectra was investigated. For this purpose the oven of the sample cuvette was filled with $3 \mathrm{~mL}$ demineralized water and set at a positive offset of $0.5 \mathrm{~K}, 1 \mathrm{~K}, 2 \mathrm{~K}, 4 \mathrm{~K}$ and $8 \mathrm{~K}$ compared to the oven of the reference cuvette also filled with $3 \mathrm{~mL}$ demineralized water and set at $(298.15 \pm 0.30) \mathrm{K}$. Five spectra were recorded when the sample oven temperature had stabilized; for the smallest offsets of $0.5 \mathrm{~K}, 1 \mathrm{~K}$ 
and $2 \mathrm{~K}$ this required 5 minutes, 15 minutes for $4 \mathrm{~K}$, and a full 60 minutes was necessary for equilibration of the $8 \mathrm{~K}$ offset, respectively.

\subsubsection{Data processing}

\section{Difference absorbance spectra}

The addition of electrolytes impacts the absorbance spectrum of demineralized water (Fig. 2.1A). Measured spectra were subtracted from that of demineralized water, to study the changes only, resulting in difference spectra (Figs. 2.1B and 2.1C). In figure 2.1D the difference absorbance spectra are multiplied by the dilution correction factor.

Data processing was performed using MATLAB 2013R. The difference absorbance around $15385 \mathrm{~cm}^{-1}(650 \mathrm{~nm})$ is the flattest in the entire wavelength range: It is far away from any electronic transition; and the absorption of demineralized water in this region is an order of magnitude smaller compared to the spectral region of $14000 \mathrm{~cm}^{-1}$ to $9091 \mathrm{~cm}^{-1}$ (714 nm to $1100 \mathrm{~nm}$ ) where the differential signals were analyzed. Therefore, each of the five spectra measured was offset corrected by zeroing the absorbance at $15385 \mathrm{~cm}^{-1}(650 \mathrm{~nm})$ and corrected for baseline drift. The results are conveniently presented in $2 \mathrm{D}$ plots. These plots were generated for each electrolyte by the following procedure:

1. The data basis was converted from wavelength in $\mathrm{nm}$ to wavenumber in $\mathrm{cm}^{-1}$.

2. Difference in absorbance (ordinate axis) was color coded from numerical values using a modified color profile version of cmap.m. [15]

3. The $2 \mathrm{D}$ plots were stacked on top of each other; beginning with the highest $\left((0.5)^{1}\right.$ $\mathrm{M})$ ending with the lowest $\left((0.5)^{5} \mathrm{M}\right)$ concentration.

4. The spectra were multiplied by the dilution factor.

Point (4) was carried out because the absorbance difference values are expected to be linearly correlated to the concentration of electrolyte according to Beer-Lambert law. Difference absorbance values of spectrum from samples of differing concentration should become identical when they are multiplied by the dilution correction factor. In reality, deviations from the Beer-Lambert law are observed (see Fig. 2.3).

\section{Frequency-wise linear regression}

When the Beer-Lambert law is applied to each frequency individually by a frequencywise linear regression (FWLR), these gross deviation can be corrected, as can be seen in figure 2.4. In detail, the $2 \mathrm{D}$ data points $(Y)$ of differential absorbance values recorded for each wavenumber $(\nu)$ and the five concentrations $(c)$ were linearly fitted using $Y(\nu, c)=a(\nu)+b(\nu) c$, where $a$ is the offset at $c=0$ and $b$ the slope of the fit. Typical values of $a$ and $b$ were found close to zero and in the order of $\pm 5 \times 10^{-3}$, respectively. 

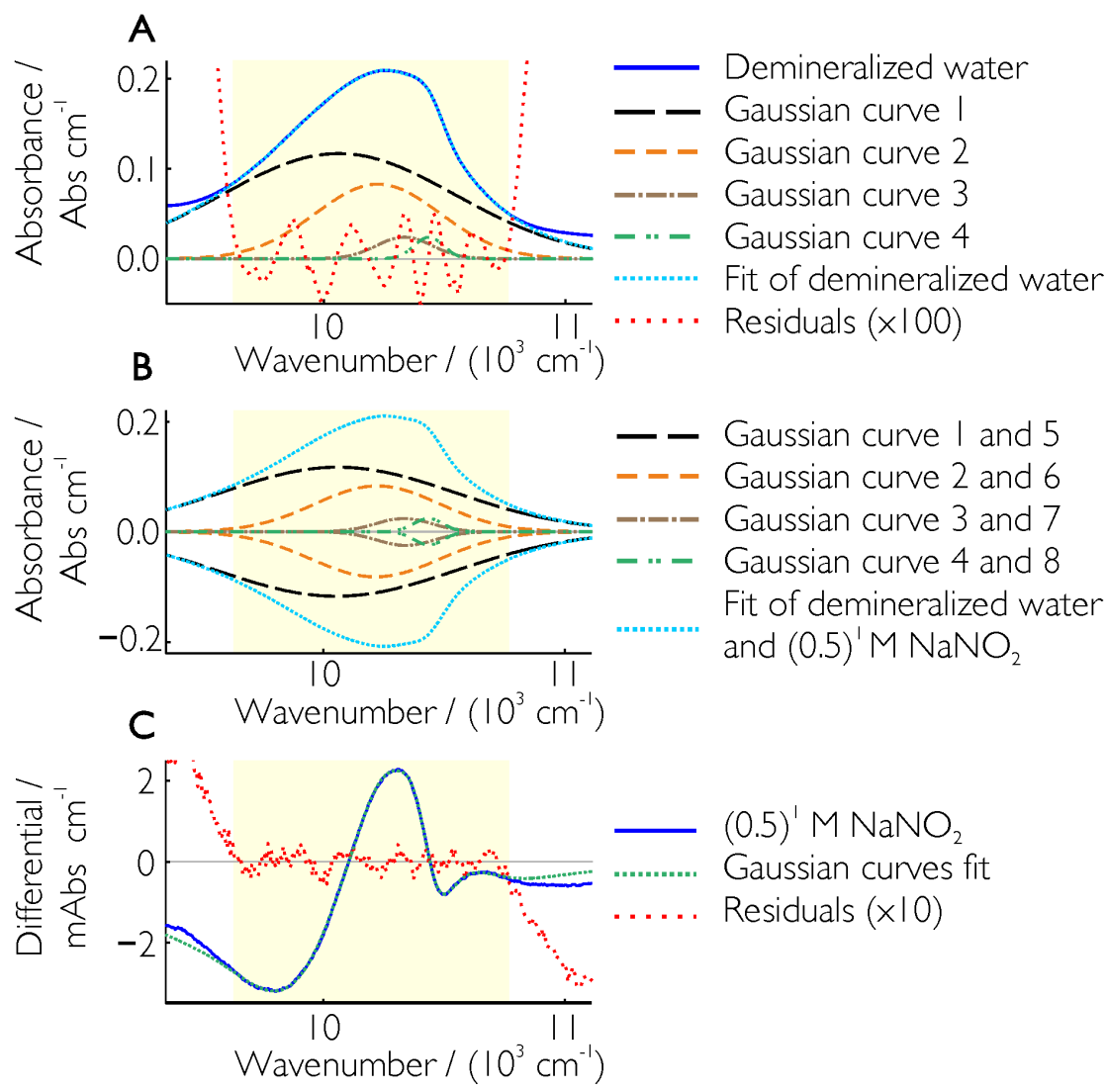

Figure 2.2: Gaussian curve fitting of differential absorbance spectrum of each electrolyte. (A) Absorbance of $1 \mathrm{~cm}$ demineralized water near $10262 \mathrm{~cm}^{-1}(974 \mathrm{~nm})$ fitted with four Gaussian curves. (B) Absorbance spectrum of water (positive curves) and absorbance spectrum of an aqueous electrolyte (inverse Gaussian curves). (C) Gaussian curve fit of the differential absorbance spectrum. The region of fitting is highlighted in yellow.

\section{Gaussian curve fitting}

GRAMS AI software was used to fit four Gaussian curves to the demineralized water absorbance in the spectral region of $10754 \mathrm{~cm}^{-1}$ to $9618 \mathrm{~cm}^{-1}(930 \mathrm{~nm}$ to $1040 \mathrm{~nm})$. Residuals in the order of $0.5 \times 10^{-3}$ were found (Fig. 2.2A) when the fitting converged to a minimum. The resulting parameters (width, position and amplitude) can be found in table 2.2. From this point on these parameters will therefore be referred to as fixed. The differential absorbance spectra of each electrolyte were fitted by adding four additional, inverse Gaussian curves (Figs. 2.2B and 2.2C). Therefore 12 parameters (width, position and amplitude) were fixed to envelop the absorbance of demineralized water and 12 parameters were fitted by the software in order to match all spectral features of the electrolytes. Width, position and amplitude of the four inverse Gaussian curves were then compared to the respective parameters of the four fixed Gaussian curves. Changes in width, position and amplitude were used to characterize the electrolytes. 
Table 2.2: Parameters of the four fixed Gaussian curves

\begin{tabular}{cccc}
\hline Gaussian & Width $\left(\mathrm{cm}^{-1}\right)$ & Position $\left(\mathrm{cm}^{-1}\right)$ & Amplitude \\
\hline$\# 1$ & 1142.7 & 10059.8 & 0.1170 \\
$\# 2$ & 610.7 & 10222.7 & 0.0829 \\
$\# 3$ & 272.4 & 10332.7 & 0.0241 \\
$\# 4$ & 143.5 & 10434.2 & 0.0240 \\
\hline
\end{tabular}

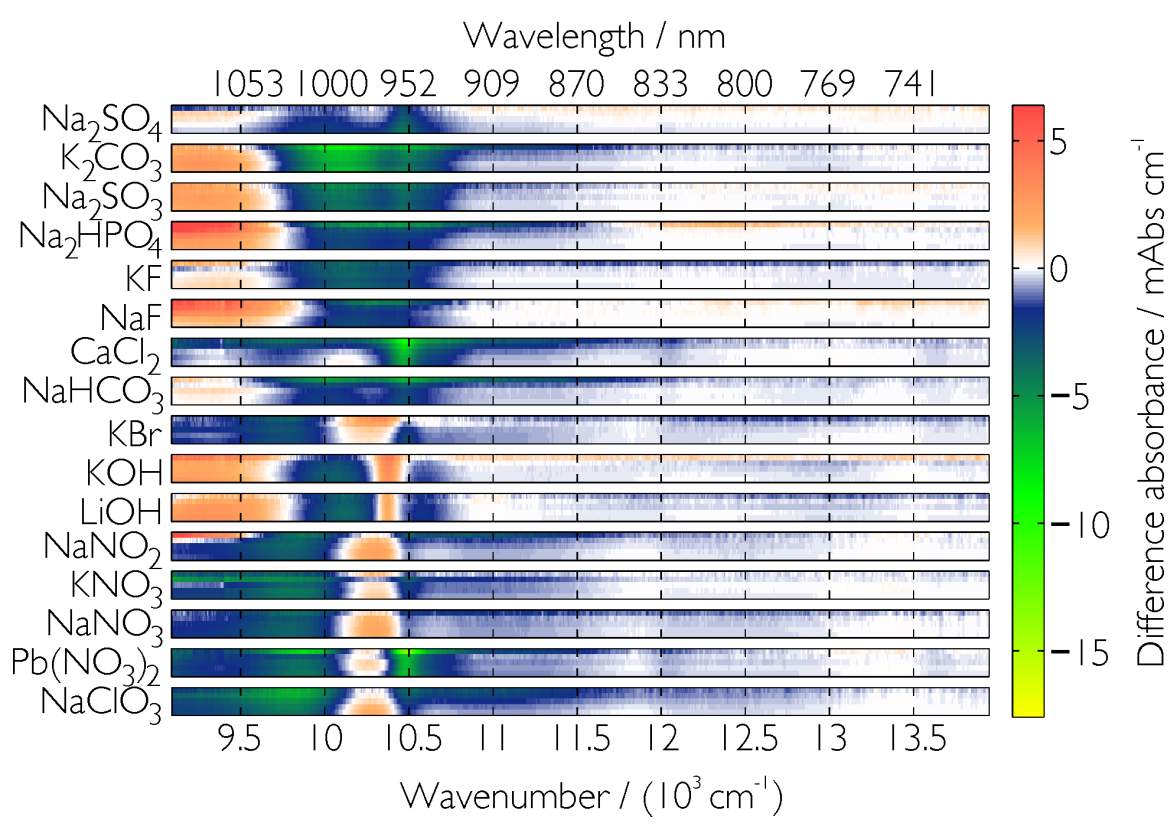

Figure 2.3: Eighty stacked 2D plots of the differential absorbance spectra for 16 salts tested at five different concentrations and a constant temperature of $(298.15 \pm 0.30) \mathrm{K}$. Deviations from Beer-Lambert law were observed even if the data is corrected using the dilution factor.

\subsection{Results}

Stacked 2D plots of the raw difference absorption spectra for each of the 16 dissolved salts are shown in figure 2.3.

\subsubsection{Frequency-wise linear regression}

In contrast to figure 2.3, the spectra corrected with the FWLR Beer-Lambert method coincide for all concentrations and thereby exhibit salt specific characteristics, as can be seen in figure 2.4. This figure shows the difference absorbance electrolyte spectra with the FWLR of the Beer-Lambert law. RMS error $0.25 \times 10^{-3}$ and of $0.1 \times 10^{-3}$ were found for the spectral region $9618 \mathrm{~cm}^{-1}$ to $9090 \mathrm{~cm}^{-1}$ and $10754 \mathrm{~cm}^{-1}$ to $9618 \mathrm{~cm}^{-1}$, respectively. As a consequence of the FWLR several isosbestic points can be identified. 
The number and location of these points is characteristic for each electrolyte allowing an easy identification of each salt.

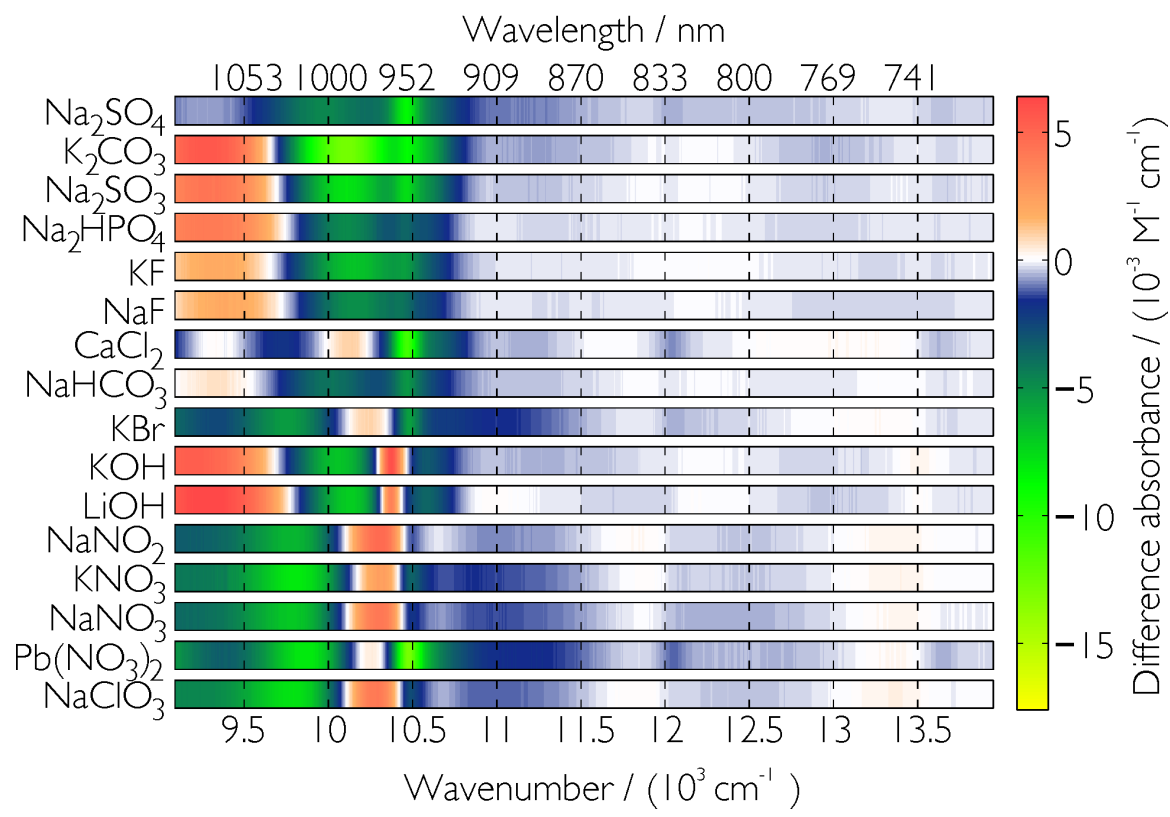

Figure 2.4: Stacked 2D plots of the differential absorbance spectra per Molar electrolyte using the FWLR Beer-Lambert method.

Increasing the temperature of pure water by $8 \mathrm{~K}$ reveals isosbestic points at $10977 \mathrm{~cm}^{-1}$ $(911.0 \mathrm{~nm})$ and $10015 \mathrm{~cm}^{-1}(998.5 \mathrm{~nm})$. The differential absorbance appears to be linear in respect to an increase of the offset temperature. Two regions have been identified: a positive region ranging from $10015 \mathrm{~cm}^{-1}$ to $9091 \mathrm{~cm}^{-1}(998.5 \mathrm{~nm}$ to $1100 \mathrm{~nm})$ with a maximum differential absorbance of $0.40 \times 10^{-3} \mathrm{~K}^{-1} \mathrm{~cm}^{-1}$ and a negative region ranging from $10977 \mathrm{~cm}^{-1}$ to $10015 \mathrm{~cm}^{-1}$ (911.0 nm to $998.5 \mathrm{~nm}$ ) with a minimum differential absorbance of $-1.27 \times 10^{-3} \mathrm{~K}^{-1} \mathrm{~cm}$. Therefore the temperature difference between sample and reference should be kept lower than $0.15 \mathrm{~K}$ in order to properly compensate any temperature effects for the lowest concentrations.

Among the anions investigated, common spectral patterns allow grouping: The patterns of the differential spectral observed for anion groups: $\mathrm{SO}_{4}{ }^{-2}, \mathrm{CO}_{3}{ }^{-2}, \mathrm{SO}_{3}{ }^{-2}, \mathrm{H}_{2} \mathrm{PO}_{4}{ }^{-}$, $\mathrm{HPO}_{4}{ }^{2-}, \mathrm{F}^{-}$and $\mathrm{HCO}_{3}{ }^{-}$exhibit similar features and are opposite of what is observed for different anion group: $\mathrm{NO}_{3}{ }^{-}, \mathrm{NO}_{2}{ }^{-}$and $\mathrm{ClO}_{3}{ }^{-}$.

\subsubsection{Gaussian curve fitting}

The change in width, position and amplitude of the fitted Gaussian curves compared to the fixed Gaussian curves due to concentration was examined using $\mathrm{Pb}\left(\mathrm{NO}_{3}\right)_{2}$ as representative example. The relative width, position and amplitude of the four moving Gaussian curves compared to the four fixed Gaussian curves are presented in figure 2.5.

A clear pattern emerges for each of the three parameters independent of concentration. 

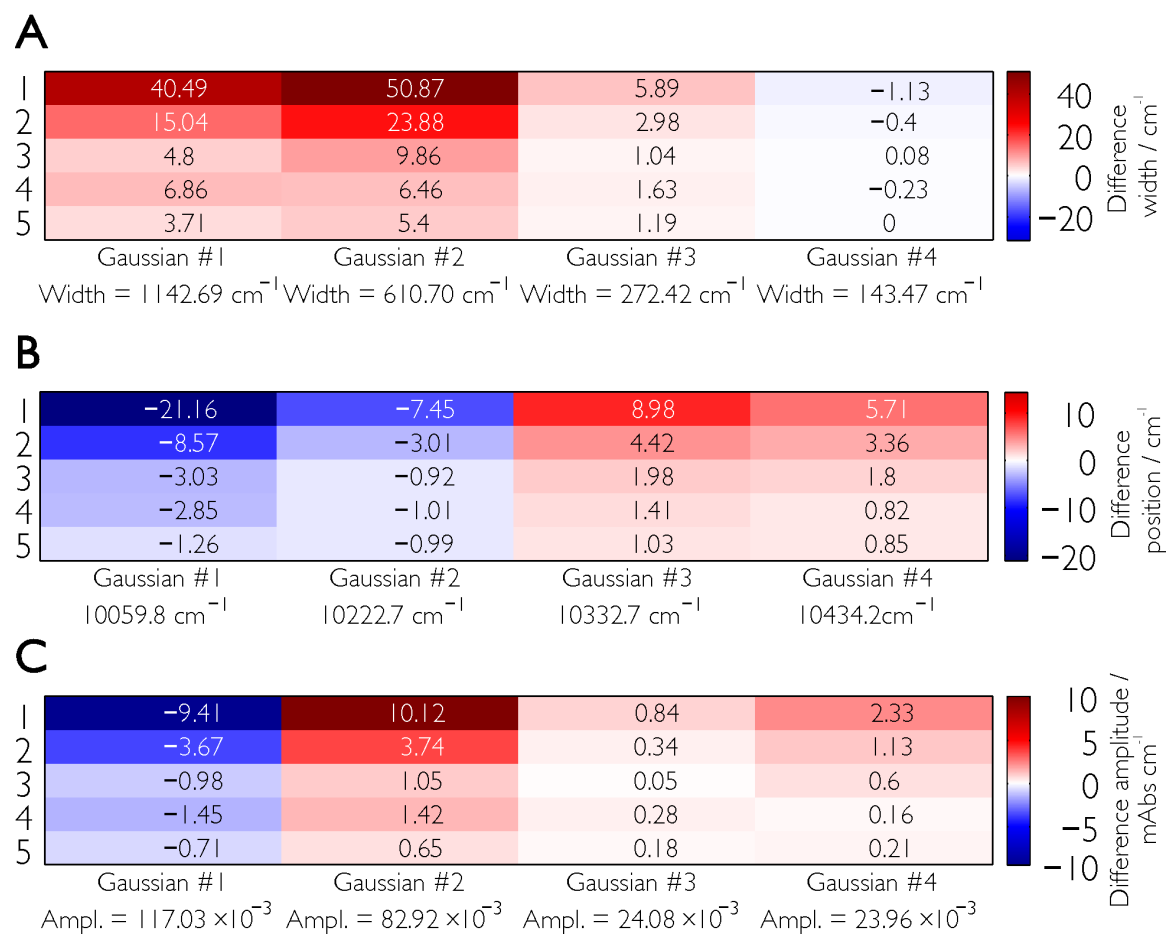

Figure 2.5: Gaussian curve fitting performed for for $0.5^{1}, 0.5^{2}, 0.5^{3}, 0.5^{4}$ and $0.5^{5} \mathrm{M} \mathrm{Pb}\left(\mathrm{NO}_{3}\right)_{2}$ (exponents given on the left of the tables) to examine the effect of concentration: (A) Difference in width, (B) difference in position, and (C) difference in amplitude.

These can be summarized as follows where $\boldsymbol{\Delta}$ indicates a positive difference and $\boldsymbol{\nabla}$ indicates a negative difference: Difference in width (A) yields pattern $\boldsymbol{\Delta} \boldsymbol{\Delta} \boldsymbol{\Delta} \boldsymbol{\nabla}$, difference in position (B) shows pattern $\boldsymbol{\nabla} \nabla \mathbf{\Delta} \boldsymbol{\Delta}$ and difference in amplitude (C) results in pattern

\section{$\boldsymbol{\nabla} \Delta \mathbf{\Delta} \Delta$.}

The effect of temperature on the fitting parameters was examined by changing the offset temperature between reference and sample cuvette filled with demineralized water. The observed pattern shown in figure 2.6 differs from the changes induced by the dissolved salts.

The pattern emerging is independent of offset temperature and can be summarized as follows where $\boldsymbol{\Delta}$ indicates a positive difference and $\boldsymbol{\nabla}$ indicates a negative difference: From difference in width (A) pattern $\boldsymbol{\Delta} \boldsymbol{\Delta} \boldsymbol{\nabla} \boldsymbol{\Delta}$ is obtained, difference in position (B) yields pattern $\Delta \boldsymbol{\Delta} \boldsymbol{\nabla} \nabla$, and difference in amplitude (C) shows the pattern $\boldsymbol{\nabla} \boldsymbol{\Delta} \Delta \boldsymbol{\Delta}$.

Figure 2.7 presents the peak fitting performed for $(0.5)^{1} \mathrm{M}$ electrolytes. Black lines separate four groups exhibiting the same patterns. Four groups exhibiting the same patterns can be summarized as follows where $\boldsymbol{\Delta}$ indicates a positive difference, $\boldsymbol{\nabla}$ indicates a negative difference and $\downarrow$ indicates a negligible difference: Difference in width (A) yields the following patterns: $\mathrm{Na}_{2} \mathrm{SO}_{4} \boldsymbol{\nabla} \boldsymbol{\nabla} \boldsymbol{\nabla} ; \mathrm{K}_{2} \mathrm{CO}_{3}-\mathrm{CaCl}_{2} \boldsymbol{\nabla} \boldsymbol{\Delta} \nabla \boldsymbol{\nabla} ; \mathrm{NaHCO}_{3}-$ $\mathrm{LiOH} \boldsymbol{\nabla} \boldsymbol{\Delta} \boldsymbol{\Delta} \boldsymbol{\nabla} ; \mathrm{NaNO}_{2}-\mathrm{NaClO}_{3} \boldsymbol{\Delta} \boldsymbol{\Delta} \boldsymbol{\Delta} \boldsymbol{\nabla}$. Difference in position results in the following patterns: $\mathrm{Na}_{2} \mathrm{SO}_{4} \boldsymbol{\nabla} \boldsymbol{\Delta} \boldsymbol{\nabla} \boldsymbol{\nabla} ; \mathrm{K}_{2} \mathrm{CO}_{3}-\mathrm{KBr} \boldsymbol{\Delta} \boldsymbol{\Delta} \boldsymbol{\nabla} \boldsymbol{\Delta} ; \mathrm{NaHCO}_{3}-\mathrm{LiOH} \boldsymbol{\Delta} \boldsymbol{\nabla} \boldsymbol{\Delta} \boldsymbol{\Delta} ; \mathrm{NaNO}_{2}-$ $\mathrm{NaClO}_{3} \boldsymbol{\nabla} \boldsymbol{\nabla} \boldsymbol{\Delta} \Delta$. Differences in amplitude (C) show the same four groups, though two 


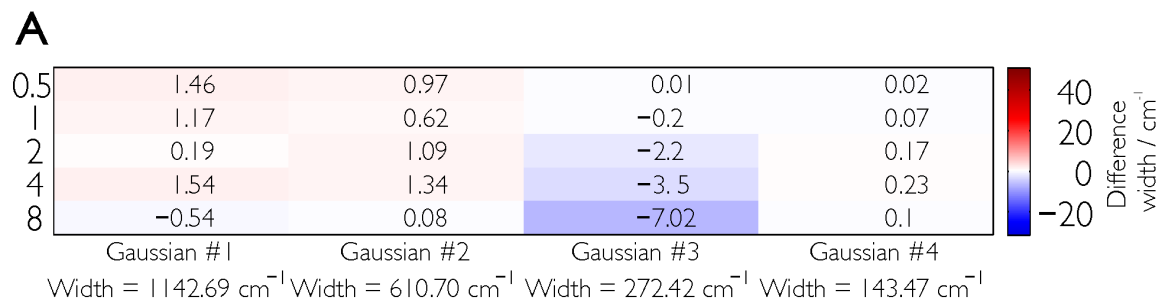

B

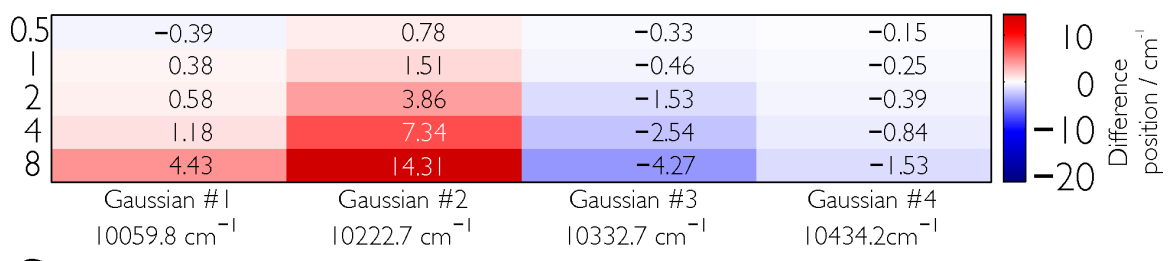

C

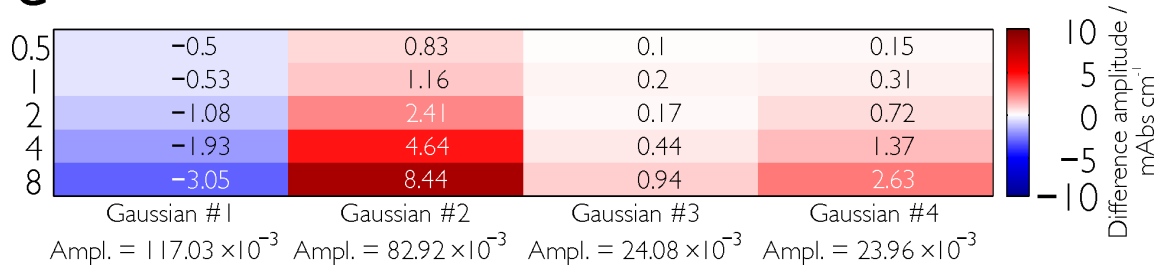

Figure 2.6: Gaussian curve fitting performed for an offset of $0.5 \mathrm{~K}, 1 \mathrm{~K}, 2 \mathrm{~K}, 4 \mathrm{~K}$ and $8 \mathrm{~K}$ (numbers on the left) between reference and sample filled with demineralized water: (A) Difference in width, (B) difference in position, and (C) Difference in amplitude.

extra groups could be identified: $\mathrm{Na}_{2} \mathrm{SO}_{4} \boldsymbol{\Delta} \boldsymbol{\Delta} \boldsymbol{\nabla} \boldsymbol{\Delta} ; \mathrm{K}_{2} \mathrm{CO}_{3}-\mathrm{NaF} \boldsymbol{\Delta} \boldsymbol{\Delta} \boldsymbol{\nabla} \boldsymbol{\Delta} ; \mathrm{NaHCO}_{3}, \mathrm{KOH}$ and $\mathrm{LiOH} \boldsymbol{\nabla} \boldsymbol{\Delta} \boldsymbol{\nabla} \mathbf{\nabla} ; \mathrm{NaNO}_{2}-\mathrm{NaNO}_{3} \boldsymbol{\nabla} \boldsymbol{\Delta} \boldsymbol{\nabla}$ and the two extra groups $\mathrm{CaCl}_{2}-\mathrm{KBr}$ $\Delta \boldsymbol{\nabla} / \Delta \boldsymbol{\Delta} ; \mathrm{Pb}\left(\mathrm{NO}_{3}\right)_{2}-\mathrm{NaClO}_{3} \boldsymbol{\nabla} \boldsymbol{\Delta} \boldsymbol{\nabla} \boldsymbol{\Delta}$.

\subsection{Discussion}

\subsubsection{Frequency-wise linear regression}

The FWLR method corrects for gross deviations from the Beer-Lambert law as can be seen by a comparison of figures 2.1 and 2.4. It is interesting to note, however, that in the region of $9618 \mathrm{~cm}^{-1}$ to $9090 \mathrm{~cm}^{-1}$ for the lowest concentration the RMS errors calculated from the fits are higher than the spectral signals, therefore these signals deviated from pure Beer-Lambert behavior. These deviations may be in part due to the physical limitation of the detection scheme, such as spectral flatness and baseline drift, and partly to the complex interactions from multiple damped oscillators which give rise to the NIR absorption bands. [16] 


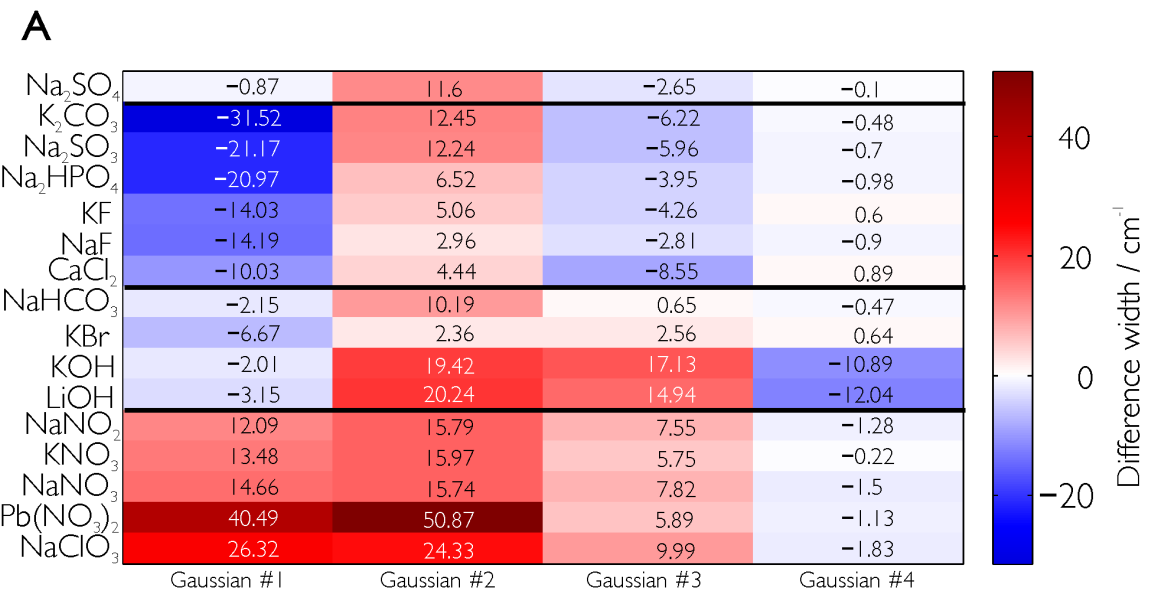

Width $=1142.69 \mathrm{~cm}^{-1}$ Width $=610.70 \mathrm{~cm}^{-1}$ Width $=272.42 \mathrm{~cm}^{-1}$ Width $=143.47 \mathrm{~cm}^{-1}$

\section{B}

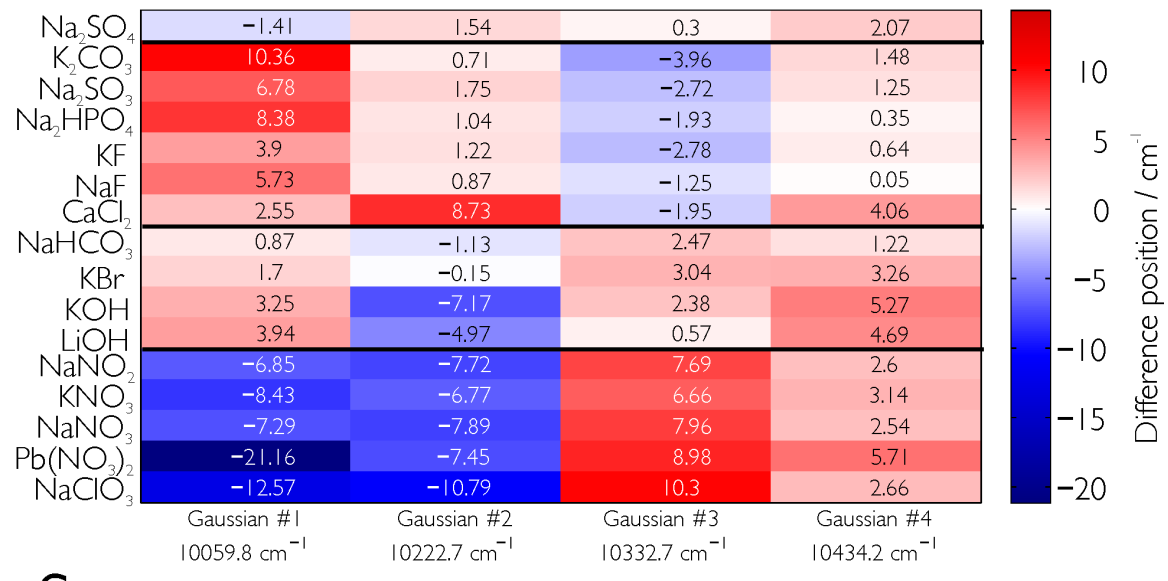

C

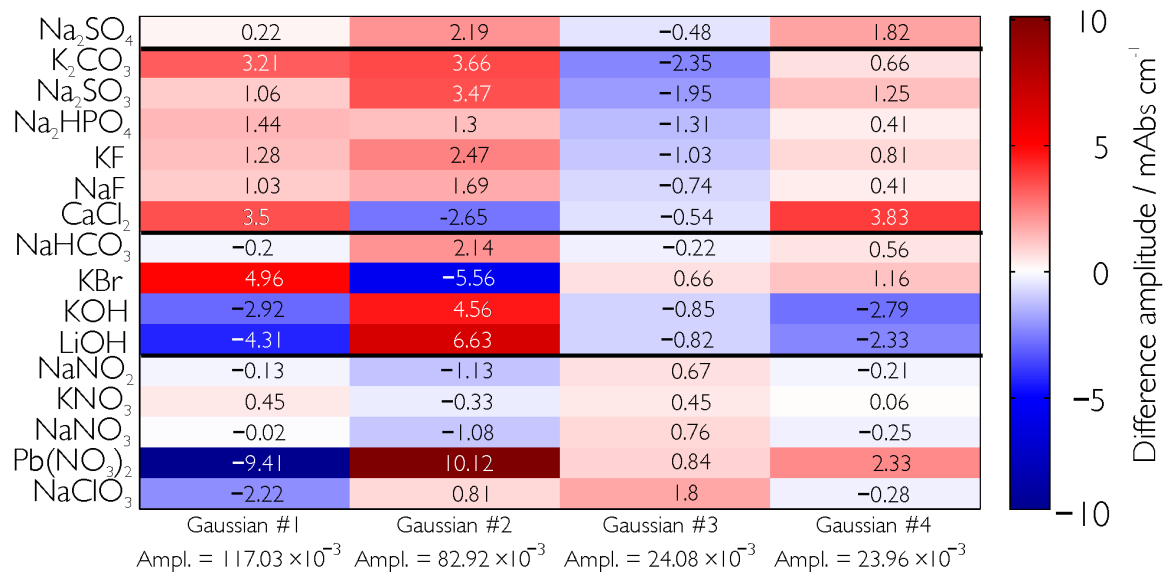

Figure 2.7: Gaussian curves fitting performed for $(0.5)^{1} \mathrm{M}$ electrolytes: (A) Difference in width, (B) difference in position, and (C) Difference in amplitude. 
Nevertheless the spectral signals of the lowest concentration were at least twice higher than the RMS errors of the fits in region $10754 \mathrm{~cm}^{-1}$ to $9618 \mathrm{~cm}^{-1}$, indicating a good fit and thus Beer-Lambert behavior. This region with good fits is suitable for sensing purposes. The method results in clear fingerprint for this region for each dissolved salt with specific location and number of isosbestic points. This fact holds the potential for a sensor to use this mathematical operation to identify electrolytes according to their isosbestic points.

The ratio of differential absorbance at different wavenumbers can be used to quantify the electrolyte. A sensor based on this fingerprint principle is limited in that it can only identify single salt species. The use of isosbestic points is most simple for single dissolved salt species, ideal environment and purity conditions. The use of this method in the identification of mixed solutions under field conditions will require future research. This course of action works well for the situations described; difficulties in compensating for the nonlinearity of the Beer-Lambert law may arise if the system is not defined a priori.

Whereas this method focusses on the application of the isosbestic points for electrolyte identification, their physical meaning is not completely understood and source of an ongoing discussion in the literature. For example, these points could be considered locations where structural species interconvert (different hydrogen bond types); an alternative explanation is given by P. L. Geissler. [17] The differential spectral features are mainly due to the anions. This larger impact of anions compared to cations was already observed by Hofmeister. [18] By comparing the differential spectra of $\mathrm{KOH}$ and $\mathrm{LiOH}$ or $\mathrm{KF}$ and $\mathrm{NaF}$ it becomes evident, however, that potassium and sodium do have a measurable influence on their environment. Therefore a sensor sensitive for both anions and cations can be built based on the findings of this work.

\subsubsection{Gaussian curve fitting}

The second method applied in this paper fits eight Gaussian curves on the absorbance in the spectral region $10754 \mathrm{~cm}^{-1}$ to $9618 \mathrm{~cm}^{-1}$ (930 nm to $1040 \mathrm{~nm}$ ) whereby four Gaussian curves are kept fixed to fit the absorbance of demineralized water and four inverse Gaussian curves are fit to get hold of the small influence ions have on the absorbance in this spectral region.

The fitting of the 8 Gaussian curves shows promising results. First, the fitted data can be used to quantify the amount of dissolved salt (Fig. 2.5). Another interesting observation is the fact that the influence of temperature has its own specific pattern as can be seen in figure 2.6. This observation can be used for temperature correction of the spectra. Furthermore, this method shows that the salts are distinguishable from each other (Fig. 2.7) and yields four groups discernible by both positional shift and change in the width of the Gaussian curves. The correlation between change in position and change in width can potentially be used to create truth tables (e.g. if $\mathrm{n}_{1}<\operatorname{signal}_{\text {width }}<\mathrm{n}_{2} \& \&$ $\mathrm{n}_{3}<$ signal $_{\text {position }}<\mathrm{n}_{4} \& \& \mathrm{n}_{5}<$ signal $_{\text {amplitude }}<\mathrm{n}_{6}$, where $\mathrm{n}_{1-6}$ are threshold values) for an aqueous electrolyte sensor. Again, the anions are more influential on the fitting parameters, but significant differences can also be seen between cations with the same anion present. 


\subsection{Conclusion}

In this study we show that 16 electrolytes can be distinguished and quantified using differential absorbance spectroscopy in the NIR wavelength region of $14000 \mathrm{~cm}^{-1}$ to $9091 \mathrm{~cm}^{-1}$ (714 nm to $1100 \mathrm{~nm}$ ) using either frequency-wise linear regression (FWLR) or Gaussian curves fitting.

The raw differential absorbance spectra are best represented by $2 \mathrm{D}$ plots since such plots have proven to be successful in comparing large amounts of spectra easily. The differential absorbance spectra multiplied with their dilution factor showed clear deviation from the Beer-Lambert law which can be corrected by the application of FWLR. This method also showed specific spectral features (isosbestic points) for each electrolyte investigated.

The Gaussian curves fitting of the narrow bandwidth spectral data of the 16 electrolytes can be converted to truth tables to be used for identification and quantification. Further research will focus on improving the SNR by increasing the path length and an optical nulling design as well as on extending the methods presented to other salts and mixtures.

This work was performed at Wetsus, European Centre of Excellence for Sustainable Water Technology (www.wetsus.nl). Wetsus is funded by the Dutch Ministry of Economic affairs, the European Union Regional Development Fund, the Province of Fryslân, the City of Leeuwarden and the EZ/Kompas program of the "Samenwerkingsverband Noord-Nederland". The authors like to thank the participants of the research theme "Sensoring" for the fruitful discussions and their financial support. We also acknowledge Jeroen Korterik for providing the electronic design of the ovens. 



\section{Bibliography}

[1] R. Suhrmann and F. Breyer, "Untersuchungen im ultraroten Absorptionsspektrum über die Änderung des Lösungsmittels durch die gelöste Substanz. I. Uber den Einfluss gelöster Salze auf den Assoziationszustand des löstenden Wassers," Z. Physik. Chem. B, vol. 20, no. 17, pp. 17-53, 1933. - p.32.

[2] G. R. Choppin and K. Buijs, "Near-infrared studies of the structure of water. ii. ionic solutions," The Journal of Chemical Physics, vol. 39, no. 8, pp. 2042-2050, 1963. - p.32.

[3] J. Lin, J. Zhou, and C. W. Brown, "Identification of Electrolytes in Aqueous Solutions from Near-IR Spectra," Applied Spectroscopy, vol. 50, pp. 444-448, 1996. - p.32.

[4] J. Lin and C. W. Brown, "Spectroscopic measurement of $\mathrm{NaCl}$ and seawater salinity in the near-IR region of 680-1230 nm," Applied Spectroscopy, vol. 47, pp. 239-241, 1993. - p..

[5] A. A. Gowen, J. M. Amigo, and R. Tsenkova, "Characterisation of hydrogen bond perturbations in aqueous systems using aquaphotomics and multivariate curve resolution-alternating least squares.," Analytica chimica acta, vol. 759, pp. 8-20, 2013. - p.32.

[6] L. Piatkowski, A. D. Wexler, E. C. Fuchs, H. Schoenmakera, and H. J. Bakker, "Ultrafast vibrational energy relaxation of the water bridge," Physical Chemistry, vol. 14, pp. 6160-6164, 2012. - p.32.

[7] G. E. Walrafen, Raman and Infrared Spectral Investigations of Water Structure, pp. 151-214. Boston, MA: Springer New York, 1972. — p.32.

[8] D. A. Schmidt and K. Miki, "Structural correlations in liquid water: A new interpretation of ir spectroscopy," The Journal of Physical Chemistry A, vol. 111, no. 40, pp. 10119-10122, 2007. - p.32.

[9] R. Rey, K. B. Møller, and J. T. Hynes, "Hydrogen bond dynamics in water and ultrafast infrared spectroscopy," The Journal of Physical Chemistry A, vol. 106, no. 50, pp. 11993-11996, 2002. - p.32.

[10] N. Chumaevskii and M. Rodnikova, "Some peculiarities of liquid water structure," Journal of Molecular Liquids, vol. 106, no. 2, pp. 167 - 177, 2003. Contribution to the Seminar on the Structure of Liquids and Liquid Solutions of the Russian Academy of Sciences in the Honour of Professor O. Ya. Samoilov, Moscow 2001. - p.32.

[11] A. W. Omta, M. F. Kropman, S. Woutersen, and H. J. Bakker, "Negligible effect of ions on the hydrogen-bond structure in liquid water," Science, vol. 301, no. 5631, pp. 347-349, 2003. - p.32.

[12] A. Gowen, F. Marini, Y. Tsuchisaka, S. D. Luca, M. Bevilacqua, C. O’Donnell, 
G. Downey, and R. Tsenkova, "On the feasibility of near infrared spectroscopy to detect contaminants in water using single salt solutions as model systems," Talanta, vol. 131 , pp. $609-618,2015$. - p.32.

[13] Shimadzu recording spectrophotometer, UV-1800, instruction manual; System User's Guide. - p.33.

[14] A. Beer, "Bestimmung der absorption des rothen lichts in farbigen flüssigkeiten," Annalen der Physik, vol. 162, no. 5, pp. 78-88, 1852. — p.34.

[15] C. Zhang, "Matlab blue-white-red colorbar, while zero always corre- sponds to white color." http: / / nl.mathworks.com/matlabcentral/fileexchange/ 36212-b2r-cmin-input-cmax-input-? focused=3869003\&tab= function. Accessed: 2012. - p.35.

[16] J. J. Loparo, S. T. Roberts, and A. Tokmakoff, "Multidimensional infrared spectroscopy of water. ii. hydrogen bond switching dynamics," The Journal of Chemical Physics, vol. 125, no. 19, p. 194522, 2006. - p.40.

[17] P. L. Geissler, "Temperature dependence of inhomogeneous broadening: On the meaning of isosbestic points," Journal of the American Chemical Society, vol. 127, no. 42, pp. 14930-14935, 2005. - p.42.

[18] F. Hoffmeiser, "Zur lehre von der wirkung der salze," Arch. Exp. Pathol. Pharmakol, vol. 24, pp. 247-260, 1888 . - p.42. 

은

(2)

1

1

$+1$

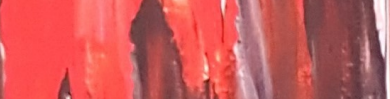

$\therefore$

a

is it

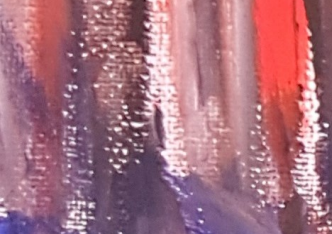

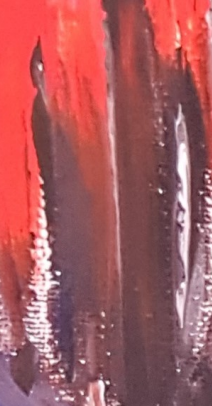

- $\log _{1}$

4

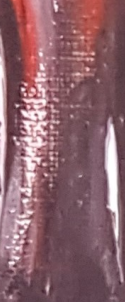

(1)
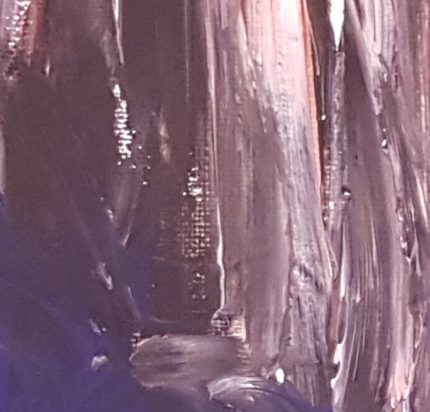

10
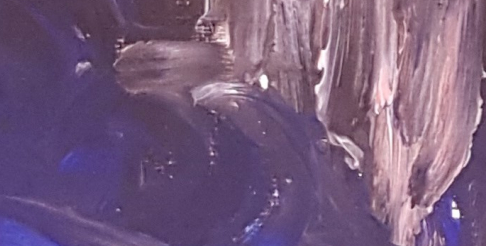

$\sqrt{1}+1$
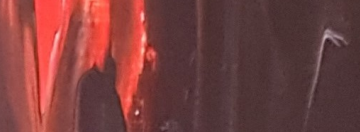

$\gamma_{3}$ ह 


\section{CHAPTER}

\section{Role of Temperature}

66 It is better to take many small steps in the right direction than to make a great leap forward to stumble backward.

Old Chinese Proverb,

This chapter has been accepted as: G. W. Steen, E. C. Fuchs, A. D. Wexler, H. A. Bakker, P. D. Nguyen, H. L. Offerhaus. "Role of temperature in de-mixing absorbance spectra composed of compound electrolyte solutions". Applied Optics. 2018. 


\subsection{Introduction}

Light has been used for centuries to probe the dynamics, structure, and anomalies of liquid water with varying degrees of success. [1] The structure of water molecules in the liquid phase, assembled by an enormous number of molecules that interact with complex hydrogen bonds forming and breaking at the picosecond timescale. [2] Numerous theories have been developed to explain the behavior of water in the liquid phase that belong to two categories: mixture and continuous models, such as free/bonded $\mathrm{OH}$ and low/high water density versus non-discrete energy distribution of H-bonds. [3-7] It has been disputed by the two views whether observed isosbestic points in absorption spectra of water (due to solutes, pressure, and temperature) are an indication of interconverting chemical/structural species or is caused by thermally equilibrated structures. [8-10] The bending angles, [11] the number, [12] the length [13] and the type [14] (single, bifurcated or trifurcated) of hydrogen bonds (H-bonds) [15] are thought to be responsible for the different vibrational modes of the $\mathrm{OH}$ bonds, and thus these features are essentially the physical origin of the spectral absorbance of liquid water.

The near-infrared (NIR) absorption spectrum of water is complex. Overtones and combinations of fundamental vibrations lead to broad overlapping absorption bands. The second overtone absorbance band of the $\mathrm{OH}$ stretch vibrations can be examined in a spectral window around $970 \mathrm{~nm}$. [16] The NIR region is suitable for on-line sensing application by probing the vibrations of the $\mathrm{OH}$ bonds. [17] Water is easily contaminated by organic carbon, bacteria, viruses, tiny dust particles and it is an excellent solvent. Solutes, temperature, and pressure are known to affect the vibrational spectra of water. $[18,19]$ Even though the physical origin of the absorbance of water in the NIR region is not yet fully understood, the focus of this work is the practical applications on the ion perturbation on the absorbance spectrum of water.

Ions induce a unique spectral change in the absorption spectrum of water with the signal exhibiting a linear dependence (Lambert-Beer law) on the concentration of the ions. Utschig et al. showed the influence of a single electrolyte, $\mathrm{NaCl}$, and seawater with temperature on the absorption spectrum of water. [20] Previous work was able to record the spectral fingerprint of 16 electrolytes. [21] Ideally, the presence of an additional dissolved salt should give a linear contribution, scaled by its respective concentration, to the total absorption cross-section. It has been discussed whether the effect of the ion, observed as a change in the absorption spectrum of water, extends beyond the first hydration shell. [22-25] Assuming the different ions only affect the adjoining water molecules the spectral fingerprints of both ions are simply superimposed upon one another. Thus the signal due to a dissolved salt $\mathrm{x}_{1}$ is unaffected by the presence of another dissolved salt $\mathrm{x}_{2}$. Nonlinear contributions to the signal might appear at high concentrations due either to direct ion-ion interaction or in the case where both salts interact with the same water molecules. At low concentrations, the fingerprint of dissolved salt is modified by the presence of another species, if and only if, one or both have vibrational coupling that extends beyond the first hydration shell. The nonlinearity of the data can be determined by fitting the absorbance value with the changing concentration (or temperature difference) for each wavelength, i.e., performing wavelength resolved linear regression (WRLR) fitting. The offset values are expected to be zero if the signal is only dependent on the variable parameter. 
In this study, the effect of temperature on the vibrational modes of demineralized water is examined at $5{ }^{\circ} \mathrm{C}, 12^{\circ} \mathrm{C}, 25^{\circ} \mathrm{C}$ and $50^{\circ} \mathrm{C}$. Furthermore, the effect of the five electrolytes $\mathrm{NaNO}_{2}, \mathrm{NaNO}_{3}, \mathrm{NaOH}, \mathrm{K}_{2} \mathrm{CO}_{3}$, and $\mathrm{CaCl}_{2}$ on the absorption water spectrum at $4{ }^{\circ} \mathrm{C}, 25^{\circ} \mathrm{C}$ and $50^{\circ} \mathrm{C}$ was probed. The highest concentration used was $1.5 \mathrm{M}$, resulting in $3 \mathrm{H}_{2} \mathrm{O}$ molecular diameter ion separation. Finally, the effect of an electrolyte mixture was studied using $\mathrm{Na}_{2} \mathrm{SO}_{4}$ and $\mathrm{NaNO}_{3}$ mixtures as well as $\mathrm{Na}_{2} \mathrm{CO}_{3}$ and $\mathrm{NaCl}$ mixtures. The highest ionic concentration used in these mixtures was $2.5 \mathrm{M}$, resulting in $2 \mathrm{H}_{2} \mathrm{O}$ molecular diameter ion separation. The near-infrared (NIR) spectral data were recorded in the spectral range from $650 \mathrm{~nm}$ to $1100 \mathrm{~nm}$.

\subsection{Methodology}

\subsubsection{Sample preparation}

$3 \mathrm{M} \mathrm{NaNO}_{2}, \mathrm{NaNO}_{3}, \mathrm{NaOH}, \mathrm{K}_{2} \mathrm{CO}_{3}$, and $4 \mathrm{M} \mathrm{CaCl}_{2}$ stock solution were prepared by using $50 \mathrm{~mL}$ ultra-pure water, a $\pm 0.1 \mathrm{mg}$ balance (PIONEER $^{\mathrm{TM}}$, Ohaus, Florham Park, NJ, USA), an ultrasonic bath, and reagent or higher grade salts (Sigma Aldrich, St Louis, MO, USA). Five serial dilutions ( $0.5 \mathrm{M}$ to $31.25 \mathrm{mM})$ were prepared from the concentrated aqueous stock solutions and were stored at $4{ }^{\circ} \mathrm{C}$ in amber glass bottles. The ionic content of the solutions was validated with a Metrohm IC Compact761 ion chromatograph (detection limits of $\pm 0.1 \mathrm{mg} \mathrm{L}^{-1}$ ), equipped with a Metrohm Metrosep A Supp4/5 Guard pre-column, Metrohm IC Compact A Supp5 150/4.0mm column and a conductivity detector. The results of the ion chromatography (IC) measurements were used for the data analysis.

For the experiments involving mixtures, $1 \mathrm{M} \mathrm{Na} 2 \mathrm{SO}_{4}, \mathrm{NaNO}_{3}, \mathrm{Na}_{2} \mathrm{CO}_{3}$ and $\mathrm{NaCl}$ stock solutions were prepared as for the other salt solutions. From these stock solutions $10 \mathrm{~mL}$ dilutions of $1 \mathrm{M}, 0.75 \mathrm{M}, 0.5 \mathrm{M}, 0.25 \mathrm{M}$ and $0.125 \mathrm{M}$ were prepared. Mixtures ( $20 \mathrm{~mL}$ total volume) were prepared from the dilutions of each possible combination. Samples were stored at room temperature in amber glass bottles.

\subsubsection{Measurement conditions}

Differential absorbance spectra were recorded with a commercial spectrophotometer (UV1800, Shimadzu, Nishinokyo-Kuwabara-cho, Kyoto, Japan) with a spectral resolution of $1 \mathrm{~nm}$ and data point resolution of $0.5 \mathrm{~nm}$. Differential absorbance (dA) is defined as the negative $\log _{10}$ of the transmission through $1 \mathrm{~cm}$ of reference liquid subtracted from the negative $\log _{10}$ of the transmission through $1 \mathrm{~cm}$ of sample liquid. According to manufacturer specifications, the root mean squared error (RMSE) noise, baseline stability, and baseline flatness are specified to be less than $0.05 \times 10^{-3},<0.3 \mathrm{mAbs} / \mathrm{h}$ (at $700 \mathrm{~nm}$ ), and $\pm 0.6 \mathrm{mAbs}$, respectively. [26] Software from the same company (UVprobe) was used to record the spectral data.

Residual organic matter in the cuvettes can influence the measurements. To avoid signal arising from cross contamination a dedicated pair of cells (634-844, Quartz SUPRASIL $®$ 300, VWR, NLD) was used. The baseline can be influenced by mechanical force on the table; this effect was reduced by placement of the set-up on a stiff plate. The $20 \mathrm{~mL}$ 
mixtures were stirred at $300 \mathrm{rpm}$ to $400 \mathrm{rpm}$ in a beaker before recording to ensure a homogeneous mixture.

Ovens (t2x2 Sport ${ }^{\mathrm{TM}} /$ Shimadzu, Quantum Northwest, Liberty Lake, WA, USA) with Peltier elements ensured $\pm 0.15^{\circ} \mathrm{C}$ temperature control. Delivered from the same company was the software T-App that was used to control the ovens. The differential absorbance (signal) was recorded at $970 \mathrm{~nm}$ and monitored to verify both samples reached a stable temperature before the differential spectra were recorded. The module with ovens did not entirely block the background light; therefore black masking tape (Thorlabs, T743-2.0) was applied to cover the gaps.

The sample holder was purged with dry nitrogen gas to prevent condensation at low temperatures. Air bubbles can scatter the light beam resulting in spikes in the recorded spectra. Stirring rods were used to stir the sample at $600 \mathrm{rpm}$ for 5 minutes to remove any bubbles after the solution reached equilibrium temperature. The cuvette (cell) remained inside the spectrograph to avoid artifacts caused by optical misalignment. The cuvettes were closed with lids to avoid evaporation.

The cuvette was flushed five times with the solution before recording to ensure previous samples did not affect the concentration of the solution. For the same reason, the recording started from the lowest concentration to the highest concentration of the solution. Furthermore, measurements were taken at least an hour after the spectrophotometer was turned on to avoid instability of the light source.

\subsubsection{Measurements procedure}

\section{Offset temperature effect}

The influence of temperature on the absorbance spectrum of water was determined by recording four datasets. To collect these datasets the following procedure was used:

1. First, both cells were filled with demineralized water and inserted in the ovens. Both ovens were held at $5{ }^{\circ} \mathrm{C}, 12^{\circ} \mathrm{C}, 25^{\circ} \mathrm{C}$ and $50^{\circ} \mathrm{C}$ for the different datasets.

2. After stabilization, the baseline was established with the spectrometer software.

3. Three spectra were recorded to record the noise floor.

4. The temperature of the oven holding the sample cell was increased, while the temperature of the reference cell was kept constant.

5. After temperature stabilization, three spectra were collected. This step was repeated for each temperature increase $\left(1^{\circ} \mathrm{C}, 2^{\circ} \mathrm{C}, 4^{\circ} \mathrm{C}\right.$ and $\left.8^{\circ} \mathrm{C}\right)$ until the highest temperature difference was reached.

6. Finally, the temperature of the sample cell was set to the initial temperature, and three spectra were recorded to observe whether instrument drift had occurred.

\section{Electrolyte effect}

The effect of $\mathrm{CaCl}_{2}, \mathrm{NaNO}_{2}, \mathrm{NaNO}_{3}, \mathrm{NaOH}$ and $\mathrm{K}_{2} \mathrm{CO}_{3}$ on the absorbance spectrum of water was determined by recording three datasets for each electrolyte. Recording 
a dataset took about an hour. These datasets were obtained by strictly following the experimental procedure below:

1. First, both cells were filled with demineralized water and inserted in the ovens. The ovens were held at $4{ }^{\circ} \mathrm{C}, 25^{\circ} \mathrm{C}$ and $50^{\circ} \mathrm{C}$ during the recordings of the data sets.

2. After stabilization, the baseline was established with the spectrometer software.

3. Three spectra of the baseline were recorded.

4. The sample cell was flushed five times by $2.8 \mathrm{~mL}$ (cell volume) sample of the solution to be measured.

5. After stabilization three spectra were collected. This step was repeated for each sample until the highest concentration $(0.48 \mathrm{M})$ was reached.

6. Finally, the cell was rinsed with distilled water five times, and fifteen spectra were recorded to observe whether instrument drift had occurred.

\section{Mixture effect}

The effect of a $\mathrm{Na}_{2} \mathrm{SO}_{4} / \mathrm{NaNO}_{3}$ mixture on the absorbance spectrum of water was determined by recording 30 data sets. These data sets were collected at five temperatures. A dataset consisted of differential spectra of the baseline (water-water), $\mathrm{NaNO}_{3}$ (fixed concentration for each dataset), solutions of $\mathrm{NaNO}_{3} / \mathrm{Na}_{2} \mathrm{SO}_{4}$ mixture $\left(\mathrm{Na}_{2} \mathrm{SO}_{4}\right.$ varied from $0 \mathrm{M}, 0.063 \mathrm{M}, 0.125 \mathrm{M}, 0.25 \mathrm{M}, 0.375 \mathrm{M}$ and $0.5 \mathrm{M}$ ) and another baseline. The concentration value of $\mathrm{NaNO}_{3}$ in the six datasets per temperature setting were $0 \mathrm{M}$, $0.063 \mathrm{M}, 0.125 \mathrm{M}, 0.25 \mathrm{M}, 0.375 \mathrm{M}$ and $0.5 \mathrm{M}$. The differential absorbance spectra of the datasets were obtained by strictly following the experimental procedure below:

1. First, both cells were filled with demineralized water and inserted in the ovens. The ovens were held at temperatures of $5^{\circ} \mathrm{C}, 15^{\circ} \mathrm{C}, 25^{\circ} \mathrm{C}, 35^{\circ} \mathrm{C}$ and $45^{\circ} \mathrm{C}$.

2. After stabilization, the baseline was established with the spectrometer software.

3. Ten spectra of the baseline were recorded.

4. The sample cell was flushed five times with the solution to be measured.

5. Fifteen spectra were collected; the last five spectra were used for processing, while the first ten spectra were used to confirm the stability of the temperature in the sample cell.

6. The above step was repeated for each sample until the highest concentration $(0.5 \mathrm{M}$ $\mathrm{Na}_{2} \mathrm{SO}_{4}$ ) was reached.

7. Finally, the cell was rinsed with distilled water five times, and fifteen spectra were recorded. The second baseline was determined from the last five spectra to observe whether instrument drift had occurred.

An full data set took about two and a half hours; a full spectral scan took about one minute. The same procedure was followed for the $\mathrm{Na}_{2} \mathrm{CO}_{3} / \mathrm{NaCl}$ mixture, for the temperatures $5^{\circ} \mathrm{C}, 25^{\circ} \mathrm{C}$ and $45^{\circ} \mathrm{C}\left(\mathrm{Na}_{2} \mathrm{CO}_{3}\right.$ varying and $\mathrm{NaCl}$ constant per dataset). 


\subsubsection{Data processing}

\section{Offset temperature effect}

The following procedure was used to analyze the temperature effect on the absorbance spectrum of demineralized water. First, the offset of each of the five recorded spectra $(N)$ was corrected by subtracting the absorbance value measured at $700 \mathrm{~nm}\left(\lambda_{\text {base }}\right)$. Second, the average of the last five spectra of the baseline and samples was determined using a custom MATLAB script (R2017b, the MathWorks, Natick, MA, USA). Finally, the differential absorbance values recorded for each wavelength were weighted and linearly fit using the differential temperature $\left(T_{d}\right)$ to determine the effect on the water absorption spectrum. Wavelength resolved linear regression (WRLR) fitting was performed for each dataset of the four baseline temperatures. The results of the WRLR analyses are presented in figure 3.4 .

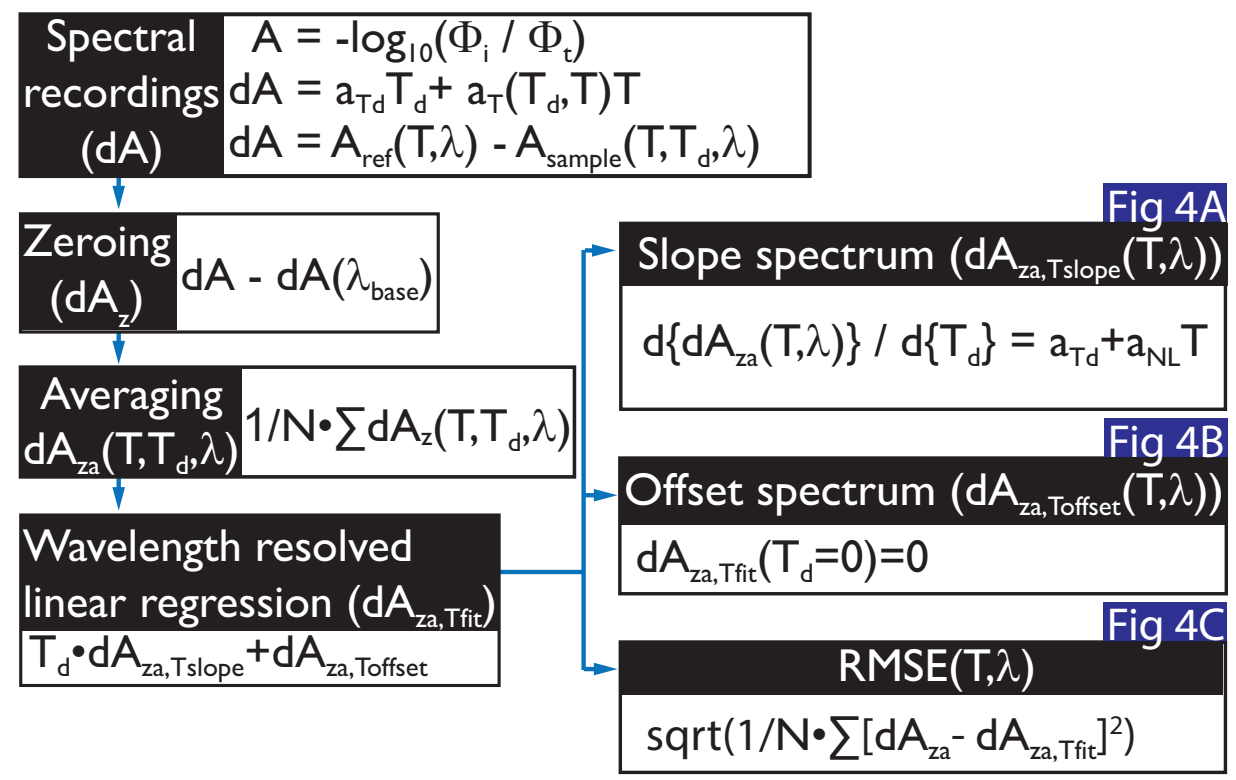

Figure 3.1: The data processing algorithm used for the offset temperature response experiments. $\Phi_{t}$ is the radiant flux transmitted, $\Phi_{i}$ is the input radiant flux. From these units the absorbance (A) is determined. The differential absorbance $(\mathrm{dA})$ is given by subtracting the absorbance spectrum of the sample from the absorbance spectrum of the reference. This is realized in the experiments by setting the base line $\left(\mathrm{A}_{\text {ref }}-\mathrm{A}_{\text {sample }}=0\right)$ with water in the reference- and sample cuvette. The outputs from the WRLR: slope, offset, and RSME are shown in figure 3.4. The slope is the change $\left(a_{d T}\right)$ of the water absorbance spectrum for a $1{ }^{\circ} \mathrm{C}$ increase at temperature $T$ and the nonlinear term $\left(\mathrm{a}_{\mathrm{NL}}\right)$. The offset values gives the change of the water absorbance unrelated to the changed parameter $\left(T_{d}\right.$, differential temperature). The RMSE values reveals the amplitude of data point deviation from a linear regression fit.

The spectral signature due to temperature increase $\left(a_{d T}+a_{T} T\right.$ in $\left.\Delta \mathrm{Abs}^{\circ} \mathrm{C}^{-1}\right)$ on the absorbance spectrum of water is given by the slope values. The RMSE values show 
non-linearity in the signal response to the temperature increase. The offset values show the background signal uncorrelated to the temperature increase.

The data algorithm is presented in figure 3.1, where $T, T_{d T}, a_{T}, a_{d T}, c, N, \Phi_{t}, \Phi_{i}$ stand for the temperature, differential temperature, temperature coefficient, differential temperature coefficient, concentration, number of spectra, radiant flux transmitted and the input radiant flux.

\section{Electrolyte effect}

A similar procedure was followed for the single dissolved salts. Three spectra were averaged instead of five, and the differential absorbance values recorded for each wavelength were similarly weighted and linearly fit using the electrolyte concentration instead of temperature. The spectral signature due to dissolved salt $\left(a_{c}+a_{T} T\right.$ in $\Delta \mathrm{Abs} \mathrm{M}^{-1}$ on the absorbance spectrum of water is given by the slope values. The offset values show the background signal uncorrelated to the changing salt concentration, for example if the sample are prepared with contaminated water. The RMSE values show non-linearity in the signal response to the ionic concentration. The results of the WRLR analyses are presented in figure 3.5 .

An additional WRLR analysis was performed on the slope (first derivative) with the three temperatures $4{ }^{\circ} \mathrm{C}, 25^{\circ} \mathrm{C}$ and $50^{\circ} \mathrm{C}$ to determine the effect of the temperature on the spectral signature of the five electrolytes. The results of the WRLR analyses are presented in figure 3.6. The ionic temperature coefficient $\left(a_{T}\right.$ in $\left.\Delta \mathrm{Abs} \mathrm{M}^{-1}{ }^{\circ} \mathrm{C}^{-1}\right)$ on the absorbance spectrum of water is given by the slope values. The offset values show the ionic coefficient $\left(a_{c}\right.$ in $\Delta \mathrm{Abs} \mathrm{M}^{-1}$ ) on the absorbance spectrum of water) at $\mathrm{T}=0^{\circ} \mathrm{C}$. The RMSE values show the robustness of the linear fit on the data by both the temperature and the concentration of an electrolyte. The data algorithm of single electrolytes is presented in figure 3.2. 


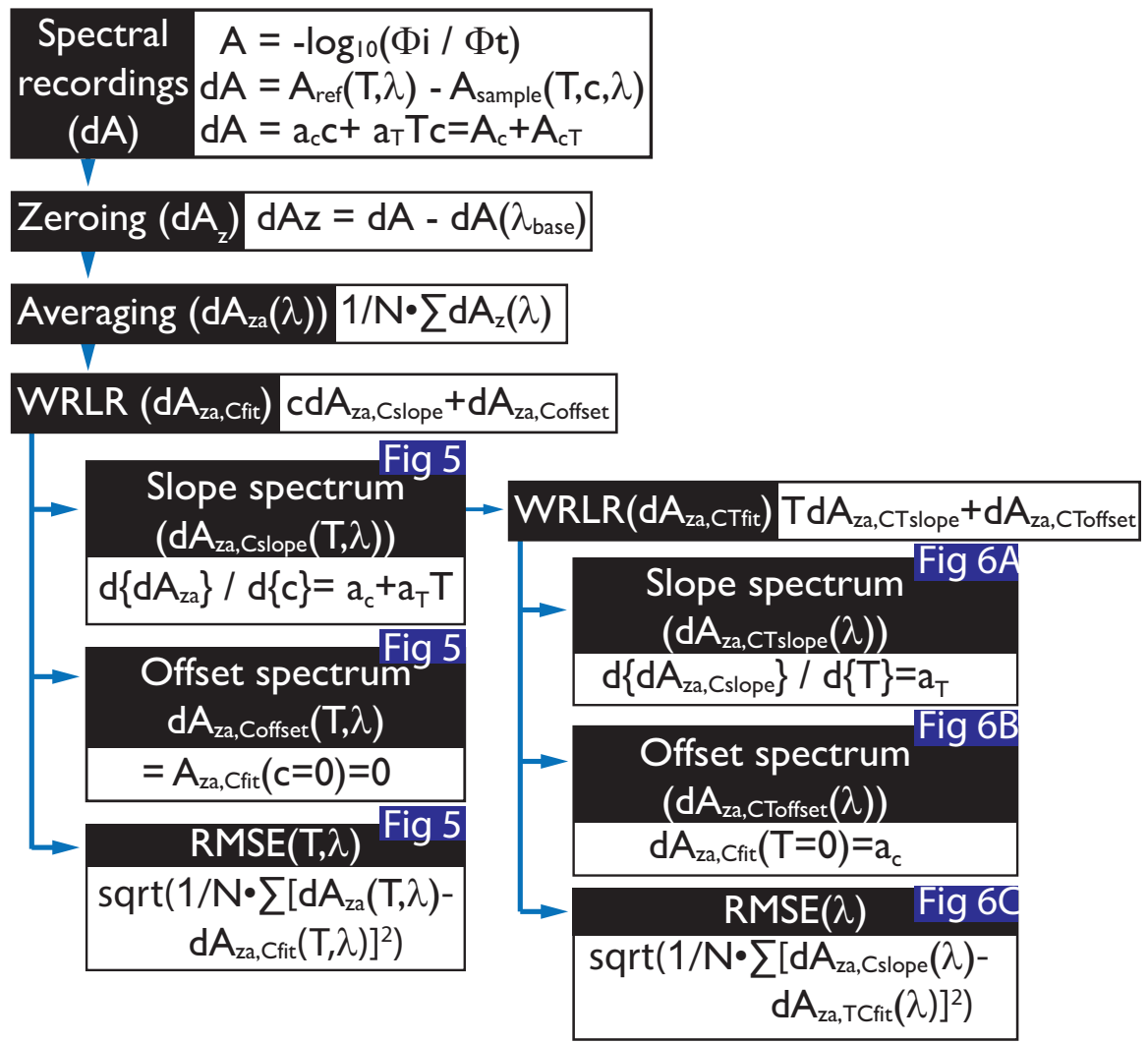

Figure 3.2: The data processing algorithm of the single electrolyte effect experiments. This algorithm provides information on the electrolyte effect combined with the temperature effect on the water absorbance spectrum. The first WRLR results in 1) the ionic coefficients at a certain temperature (slope), 2) The offset gives the change of the water absorbance unrelated to the changed concentration of the single electrolyte and 3) the RMSE gives the amplitude of the data spread around the linear regression fit. The output of the first WRLR is presented in figure 3.5. The second WRLR results in 1) the temperature coefficients (slope), 2) the ionic coefficients (offset) and 3) the RMSE gives the amplitude of the data point deviations from the linear regression fit and is presented in figure 3.6. 


\section{Mixture effect}

Another modified procedure was used to analyze the spectral data from the salt mixtures:

1. First, the offset of each of the five recorded spectra was corrected by subtracting the absorbance value measured at $650 \mathrm{~nm}$ from the entire spectra dataset.

2. The average of the last five spectra of the baselines and samples was determined by a custom MATLAB script.

3. The instrument drift was determined by assuming a linear shift over time between the first and second baseline. The average spectra of the samples were corrected for this linear drift.

4. The differential absorbance values recorded for each wavelength were weighted and linearly fit using the concentration of the varying $\mathrm{Na}_{2} \mathrm{SO}_{4}$ to determine the solute effect on the water absorption spectrum. The WRLR fitting was performed for the 30 data sets. The slope values give the $\mathrm{Na}_{2} \mathrm{SO}_{4}$ ionic coefficient $\left(a_{1}\right)$ with the nonlinear coefficient $\left(a_{3}\right)$ times the concentration of $\mathrm{NaNO}_{3}\left(c_{2}\right)$. The offset values show the spectral signature due to the dissolved $\mathrm{NaNO}_{3}\left(A_{2}\right)$ on the absorbance spectrum of water. The RMSE values show non-linearity in the signal response to this ionic concentration variation. Due to temperature control uncertainty, some recordings were rejected.

5. Finally, an additional WRLR analysis was performed on both the slope (first derivative) and offset (residual) values from the collected spectra with the varying concentration of $\mathrm{NaNO}_{3}$ between the datasets (Fig. 3.7). The slope-, and offset values of the WRLR analysis on the first derivative are the nonlinear coefficients $\left(a_{3}\right)$, and the $\mathrm{Na}_{2} \mathrm{SO}_{4}$ ionic coefficient $\left(a_{1}\right)$, respectively. The slope values of the WRLR analysis on the residuals are the $\mathrm{NaNO}_{3}$ ionic coefficient $\left(a_{2}\right)$. The RMSE values show the quality of the linear fits.

The same procedure was used to determine the effect of a constant $\mathrm{NaCl}$ on the spectral signature of $\mathrm{Na}_{2} \mathrm{CO}_{3}$ (figure 3.8). 


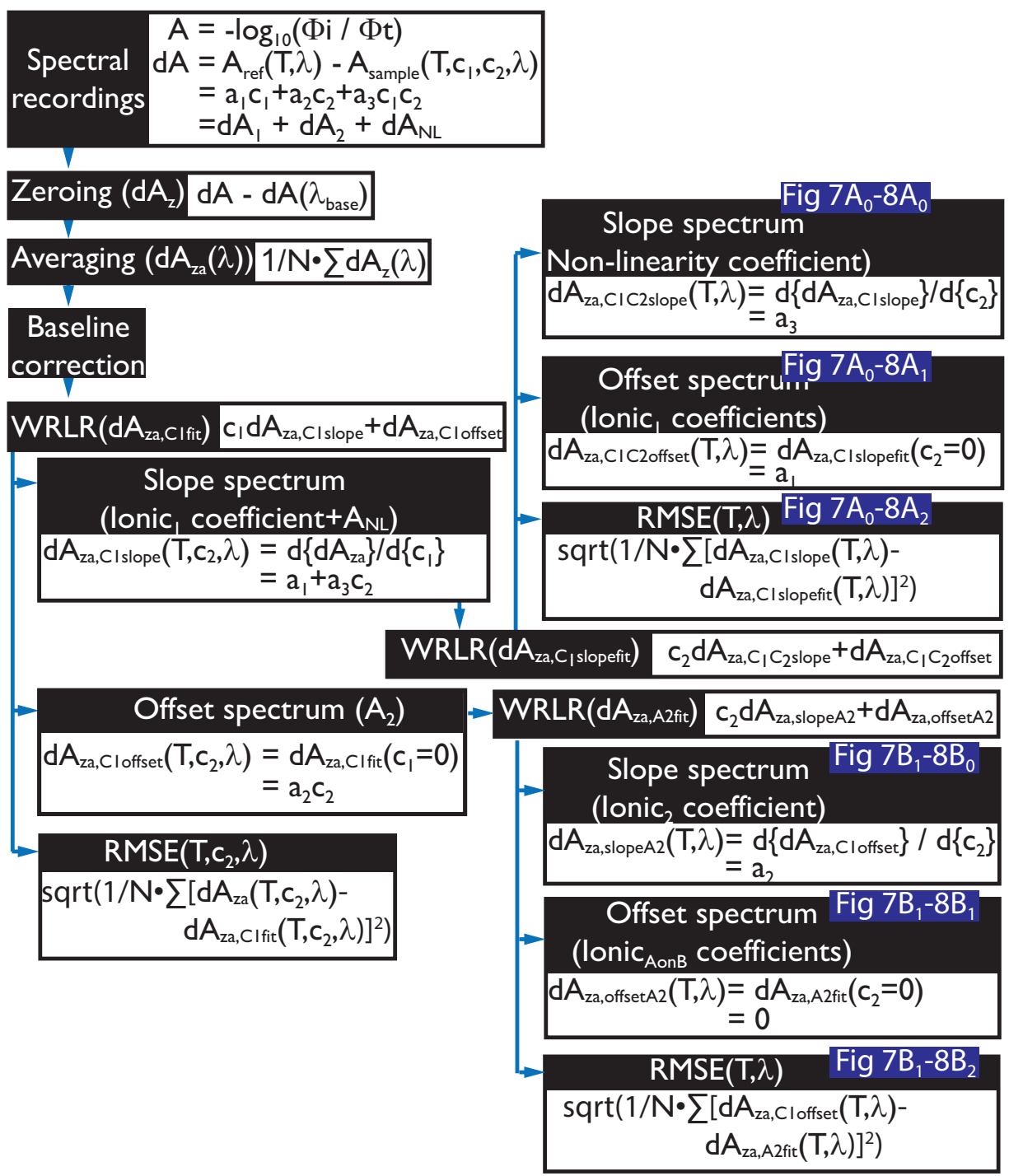

Figure 3.3: The data processing algorithm used for the electrolyte mixture experiments. This processing algorithm provides information on the electrolyte mixtures. The first WRLR results in 1) the first ionic coefficients with a nonlinear contribution of the mixture (slope), 2) The offset gives the change of the water absorbance related to the second ionic concentrations and 3) the RMSE gives the amplitude of the data spread around the linear regression fit. The second WRLR on the slope spectra results in 1) the non-linearity (slope), 2) the first ionic coefficients (offset) and 3) the RMSE values gives the amplitude of the data-point deviations from the linear regression fit and are presented. The second WRLR on the offset spectra results in 1) the second ionic coefficients (slope), 2 ) the offset (offset) and 3) the RMSE values gives the amplitude of the data-point deviations from the linear regression fit and are presented in figure 3.7 and 3.8 as indicated in the diagram. Supplemental figures are presented in appendix A. 


\subsection{Results \& Discussion}

\subsubsection{Offset temperature effect}

The effect of temperature on the differential NIR absorption of water is shown in figure 3.4. Temperature increase causes in most cases the hydrogen bonds to weaken, resulting in strengthening the covalent $\mathrm{OH}$ bonds and a higher vibrational frequency. [27] The slope values $\left(\mathrm{a}_{\mathrm{Td}}+\mathrm{a}_{\mathrm{T}}\right)$ of the WRLR analysis shows positive values for shorter wavelengths and negative values for longer wavelengths, indicating the expected blueshift of the absorbance spectrum of water. The fitted slope values are highest for the lowest temperature and decrease with temperature. This observation is marked by I in the figure with a solid blue arrow. A temperature increase at a higher temperature may increase the strengthening effect on the $\mathrm{OH}$-bond as the temperature coefficient at different temperatures appear to blue-shift (indicated in the figure with a black dashed arrow). However one could also argue that the reduced intensity of the stretching bands with increasing temperature causes the spectra to appear to spectrally blue-shift.

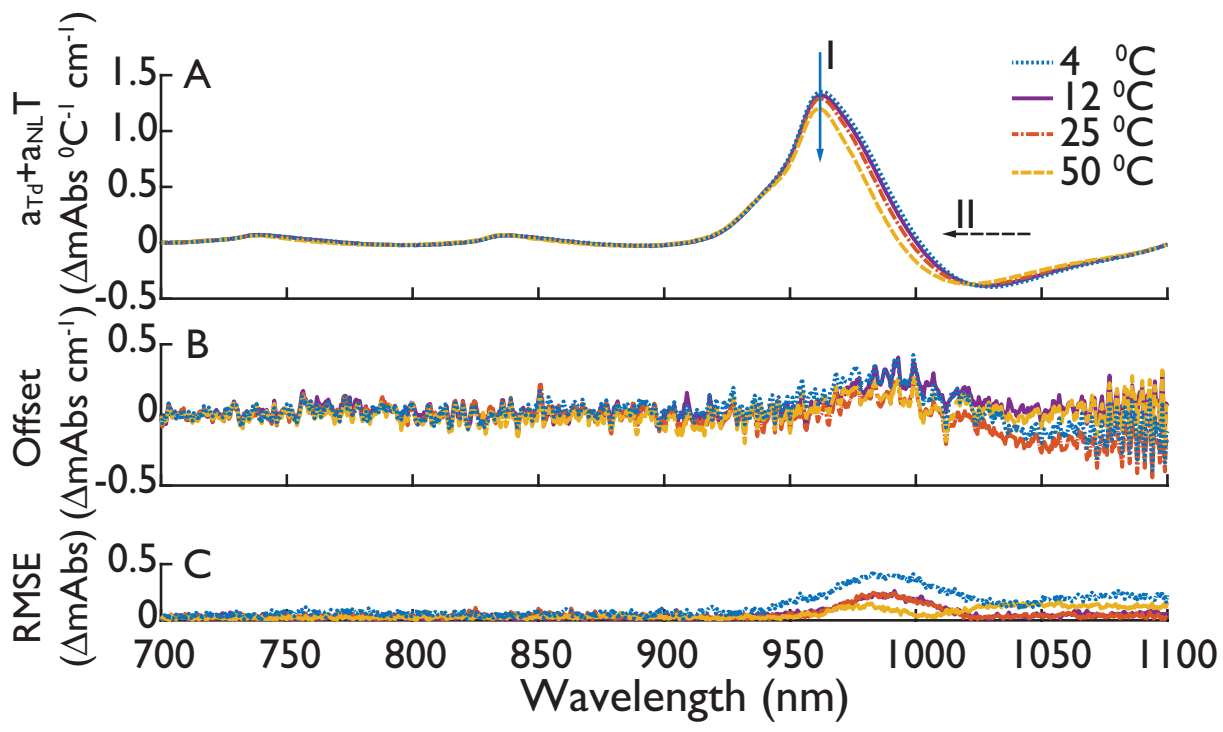

Figure 3.4: The effect of temperature at $5{ }^{\circ} \mathrm{C}, 12{ }^{\circ} \mathrm{C}, 25^{\circ} \mathrm{C}$ and $50{ }^{\circ} \mathrm{C}$ on the absorbance spectrum of demineralized water. The slope, offset, and non-linearity (RMSE) obtained from WRLR analysis are shown in subfigure $\mathrm{A}, \mathrm{B}$, and $\mathrm{C}$, respectively. The solid blue arrow I, and dashed black arrow II mark the decreases in the intensity of the slope values with increase in temperature, and the potential spectral shift due to temperature, respectively.

The offset spectra show a consistent small spectral feature. The $\pm 0.3{ }^{\circ} \mathrm{C}$ uncertainty of the temperature control can contribute to the small observed spectral feature in the offset and RMSE spectra. With rising temperature, the RMSE values around $970 \mathrm{~nm}$ decreases. The deviations are smaller than the $0.6 \mathrm{mAbs}$ flatness of the baseline. 


\subsubsection{Single electrolyte effect}

The WRLR analyses on the differential absorbance spectra of the five electrolytes at different temperatures are presented in figure 3.5. Each of the five electrolytes influences the water absorbance spectrum uniquely as can be seen from the ionic coefficient spectra. This spectral signature of the dissolved salts depends on the temperature. A common trend is observed, the ionic coefficient in the $900 \mathrm{~nm}$ to $980 \mathrm{~nm}$ region show negative values at higher temperatures, while the longer wavelength show (smaller) positive values. The electrolytes $\mathrm{NaNO}_{2}$ and $\mathrm{NaNO}_{3}$ have the same ionic coefficients in the spectral region of $900 \mathrm{~nm}$ to $950 \mathrm{~nm}$ with the different temperatures. The $\mathrm{NO}_{2}{ }^{-}$and $\mathrm{NO}_{3}{ }^{-}$ions have different ionic coefficients in the spectral region of $950 \mathrm{~nm}$ to $1100 \mathrm{~nm}$.
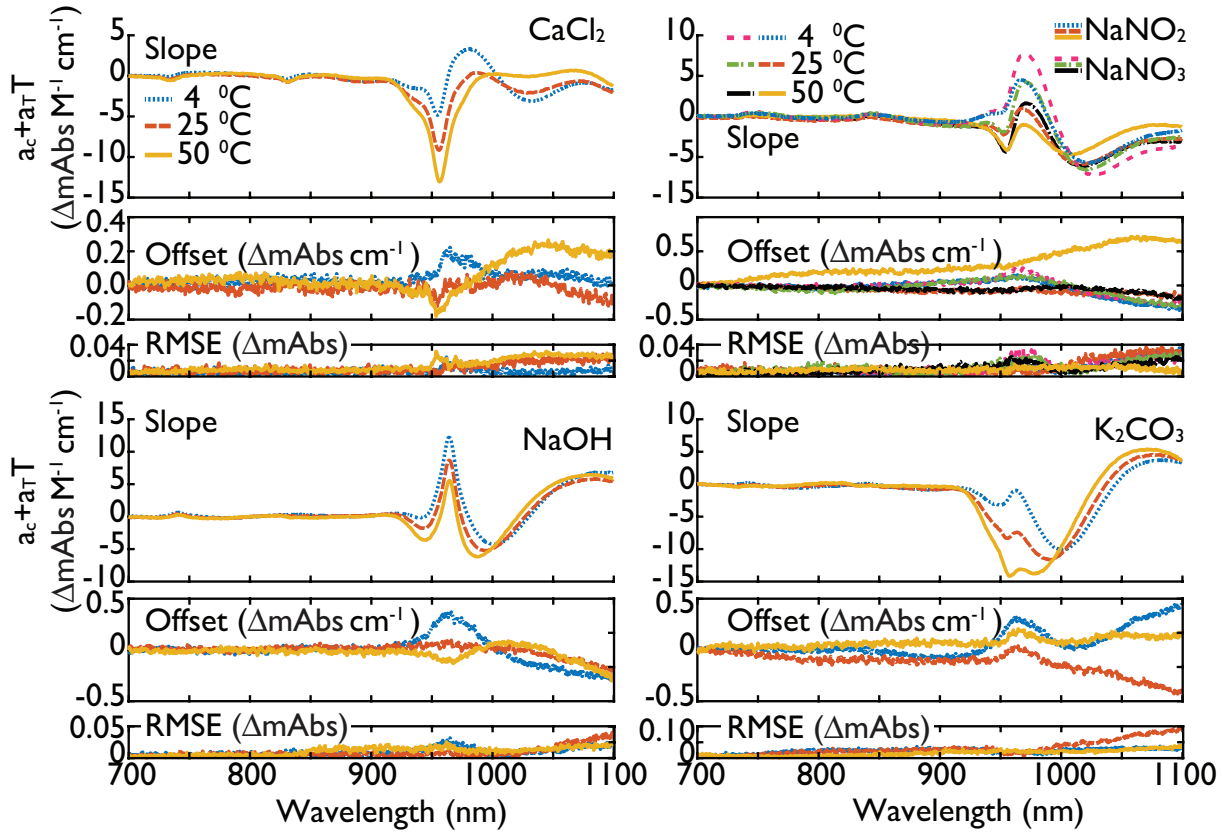

Figure 3.5: These plots compare the WRLR spectra of the different electrolytes used in this study. The additional effect temperature has on the water absorbance spectrum is shown by plotting the analyses on the three temperatures. The WRLR analysis of $\mathrm{NaNO}_{2}$ and $\mathrm{NaNO}_{3}$ are plotted together to aid visual comparison.

The offset values are expected to be zero, indicating the signal to be only dependent on the varying electrolyte concentration. The fitted offset values increases at wavelengths higher than $1000 \mathrm{~nm}$. For some datasets the offset and RMSE values peak around $955 \mathrm{~nm}$. The data points recorded around these wavelengths lay between the minimum and maximum absorption values, resulting in a higher uncertainty. This unfortunately also affects the positions of the isosbestic points that would otherwise be suitable for identification of different electrolytes. The deviation around $955 \mathrm{~nm}$ could also originate from a nonlinear contribution. 
The presented WRLR analyses yielded RMSE values smaller than $0.04 \times 10^{-3}$, except for one dataset $\left(\mathrm{K}_{2} \mathrm{CO}_{3} 25^{\circ} \mathrm{C}, 0.1 \times 10^{-3}\right)$, and offset values smaller than $0.5 \mathrm{mAbs}$. These values are smaller than the given instrument accuracy, supporting the linear fit. The root mean squared error (RMSE) values increased with wavelength. This can be explained by the decreasing quantum efficiency of the silicon array detector, also increase of light scattering due to the concentration of the electrolyte may contribute to the increasing RMSE. [28] The higher RSME value $\left(0.1 \times 10^{-3}\right)$ found in the analysis of the $\mathrm{K}_{2} \mathrm{CO}_{3}$ at $25{ }^{\circ} \mathrm{C}$ might have been caused by air bubbles present despite measures taken to avoid them. Variation in dissolved gas concentration is another possible reason for the drift. [29]

The additional WRLR analysis on the electrolyte signature with temperature shows the effect of temperature on the spectral features (ionic coefficient) caused by the ions. The result of the analyses is presented in figure 3.6. Each electrolyte influences the liquid environment differently (the offset, ionic coefficient), but have similar temperature responses (the slope, temperature coefficient). The temperature coefficients of $\mathrm{NaNO}_{2}$, $\mathrm{NaNO}_{3}, \mathrm{NaOH}$, and $\mathrm{CaCl}_{2}$ are half as big than those of $\mathrm{K}_{2} \mathrm{CO}_{3}$ and might be explained by the charge of the anion. The temperature coefficients show that the dissolved salts shift the absorbance spectrum of water to the red. The presence of ions in water, therefore, reduces the effect of increasing temperature on the absorbance spectrum of water. The deceleration of water rotation could be largely due to the coupling of the slow, collective component of water rotation with the motion of large hydrated ion clusters in concentrated ionic solutions, as proposed by Wei Zhuang et al. [30] However, their simulation used high $(>1 \mathrm{M})$ ionic solutions.
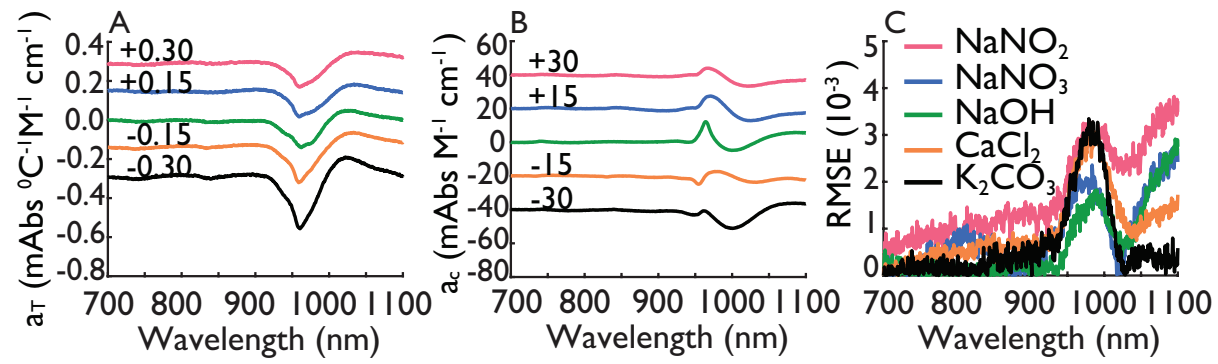

Figure 3.6: This plot presents the slope (A, temperature coefficient), offset (B, ionic coefficients), and RMSE $(\mathrm{C})$ of the additional WRLR analysis, showing the effect of temperature and ions on the absorbance spectrum of water.

\subsubsection{Mixture effect}

Figure 3.7 shows the effect that $\mathrm{NaNO}_{3}\left(B_{0}\right)$, and electrolyte $\mathrm{Na}_{2} \mathrm{SO}_{4}\left(A_{l}\right)$ have on the absorbance spectrum of demineralized water. The de-mixing of spectral signals due to $\mathrm{NaNO}_{3}$ and $\mathrm{Na}_{2} \mathrm{SO}_{4}$ on the absorbance spectrum of water is quantified with the non-linearity coefficient $\left(A_{0}\right)$. The temperature dependence on de-mixing effect can be observed by the spectral feature observed at $954 \mathrm{~nm}$. At higher temperatures, the intensity of the feature diminishes. A similar trend was observed for the spectral analysis on the 
$\mathrm{Na}_{2} \mathrm{CO}_{3}$ and $\mathrm{NaCl}$ mixture (Fig. 3.8). The dynamic movement of water molecules may be the physical reason for the de-mixing role of temperature. The nonlinear coefficients determined for both mixtures have similar spectral shape and amplitude. A follow-up study is required to determine whether the nonlinear terms are ion species independent. The linear fits on the recorded absorbance spectra of the single electrolytes (recorded at 25 degrees Celsius) seem to support this. The expected nonlinear contributions of $\sim 0.4 \mathrm{mAbs}\left(0.25 \mathrm{M}\right.$ x $\left.0.25 \mathrm{M} \mathrm{x}-6 \mathrm{mAbsM}^{-2}\right)$ can explain the spectral features seen around $954 \mathrm{~nm}$.
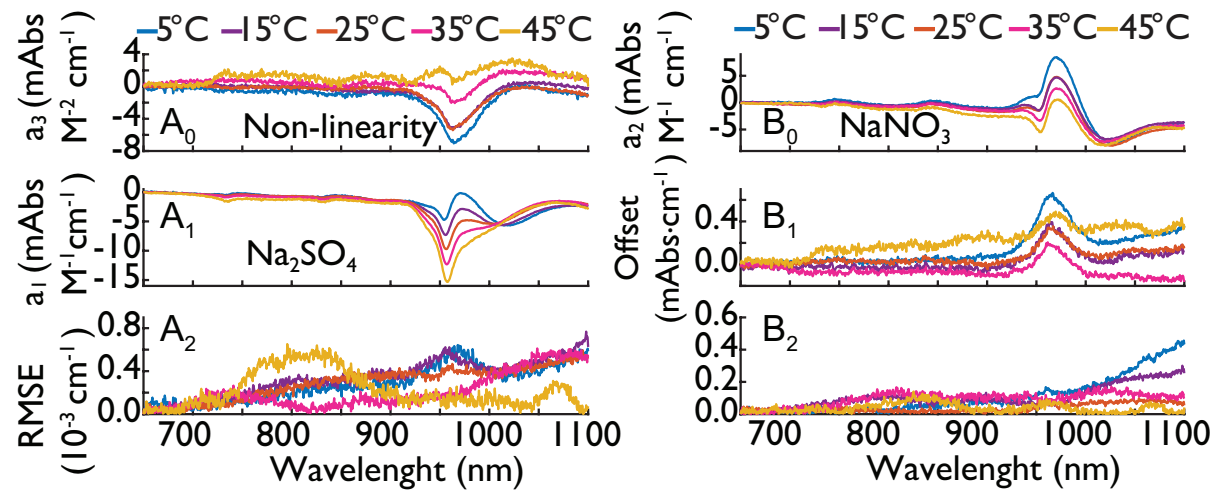

Figure 3.7: The non-linearity (A0), the coefficient of $\mathrm{NaNO}_{3}$ (A1) the RMSE values (A2), the coefficient of $\mathrm{Na}_{2} \mathrm{SO}_{4}$ (B0), the offset values (B1) and the RMSE values (B2) at $5{ }^{\circ} \mathrm{C}, 15^{\circ} \mathrm{C}, 25{ }^{\circ} \mathrm{C}$, $35^{\circ} \mathrm{C}$ and $45^{\circ} \mathrm{C}$.
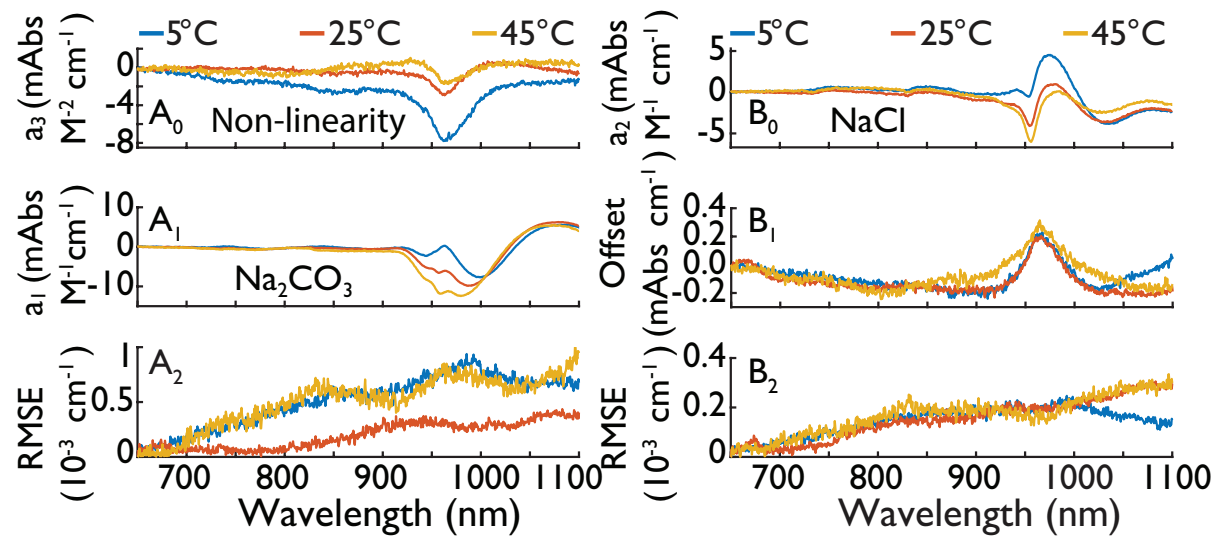

Figure 3.8: The non-linearity (A0), the coefficient of $\mathrm{Na}_{2} \mathrm{CO}_{3}(\mathrm{~A} 1)$ the RMSE values (A2), the coefficient of $\mathrm{NaCl}$ (B0), the offset values (B1) and the RMSE values (B2) at $5{ }^{\circ} \mathrm{C}, 25^{\circ} \mathrm{C}$ and $45^{\circ} \mathrm{C}$ 


\subsection{Conclusion}

The vibrational modes of water were measured in terms of differential absorption spectra at different temperatures for several electrolytes and some of their combinations. The following conclusions can be drawn from the WRLR analyses of the obtained spectra.

- Signal changes due to recording at different temperatures appear to be unrelated to the ionic species, with the exception of $\mathrm{K}_{2} \mathrm{CO}_{3}$.

- Ions affect the hydrogen bond network beyond their first hydration shell up to approx. 2 layers of water. This conclusion can be drawn from the nonlinear effects found in electrolyte mixtures and high concentrations.

- The intensity of the nonlinear contribution can be weakened by sensor operation at higher temperature.

This work was performed at Wetsus, European Centre of Excellence for Sustainable Water Technology (www.wetsus.nl). Wetsus is funded by the Dutch Ministry of Economic affairs, the European Union Regional Development Fund, the Province of Fryslân, the City of Leeuwarden and the EZ/Kompas program of the "Samenwerkingsverband Noord-Nederland". The authors like to thank the participants of the research theme "Sensoring" for the fruitful discussions and their financial support. We mourn the loss of Dhin Phong Nguyen who was taken from us on the first of November 2017. 



\section{Bibliography}

[1] P. Ball, "Water: water-an enduring mystery," Nature, vol. 452, no. 7185, p. 291, 2008. - p.50.

[2] E. Cornicchi, G. Onori, and A. Paciaroni, "Picosecond-time-scale fluctuations of proteins in glassy matrices: the role of viscosity," Physical review letters, vol. 95, no. 15, p. 158104,2005 . - p.50.

[3] F. N. Keutsch and R. J. Saykally, "Water clusters: untangling the mysteries of the liquid, one molecule at a time," Proceedings of the National Academy of Sciences, vol. 98, no. 19, pp. 10533-10540, 2001. - p.50.

[4] B. Guillot, "A reappraisal of what we have learnt during three decades of computer simulations on water," Journal of Molecular Liquids, vol. 101, no. 1-3, pp. 219-260, 2002. - p..

[5] P. M. Wiggins, "High and low density intracellular water.," Cellular and molecular biology (Noisy-le-Grand, France), vol. 47, no. 5, pp. 735-744, 2001. - p..

[6] T. D. Kühne and R. Z. Khaliullin, "Electronic signature of the instantaneous asymmetry in the first coordination shell of liquid water," Nature communications, vol. 4, p. 1450, 2013. - p..

[7] J. D. Smith, C. D. Cappa, K. R. Wilson, R. C. Cohen, P. L. Geissler, and R. J. Saykally, "Unified description of temperature-dependent hydrogen-bond rearrangements in liquid water," Proceedings of the National Academy of Sciences of the United States of America, vol. 102, no. 40, pp. 14171-14174, 2005. — p.50.

[8] P. L. Geissler, "Temperature dependence of inhomogeneous broadening: On the meaning of isosbestic points," Journal of the American Chemical Society, vol. 127, no. 42, pp. 14930-14935, 2005. - p.50.

[9] A. Nilsson and L. G. Pettersson, "The structural origin of anomalous properties of liquid water," Nature communications, vol. 6, p. 8998, 2015. - p..

[10] T. Morawietz, O. Marsalek, S. R. Pattenaude, L. M. Streacker, D. Ben-Amotz, and T. E. Markland, "The interplay of structure and dynamics in the raman spectrum of liquid water over the full frequency and temperature range," The journal of physical chemistry letters, vol. 9, no. 4, pp. 851-857, 2018. - p.50.

[11] R. Rey, K. B. Møller, and J. T. Hynes, "Hydrogen bond dynamics in water and ultrafast infrared spectroscopy," The Journal of Physical Chemistry A, vol. 106, no. 50, pp. 11993-11996, 2002. - p.50.

[12] G. E. Walrafen, Raman and Infrared Spectral Investigations of Water Structure, pp. 151-214. Boston, MA: Springer New York, 1972. — p.50.

[13] D. A. Schmidt and K. Miki, "Structural correlations in liquid water: A new interpretation of ir spectroscopy," The Journal of Physical Chemistry A, vol. 111, no. 40, pp. 10119-10122, 2007. - p.50. 
[14] N. Chumaevskii and M. Rodnikova, "Some peculiarities of liquid water structure," Journal of Molecular Liquids, vol. 106, no. 2, pp. 167 - 177, 2003. Contribution to the Seminar on the Structure of Liquids and Liquid Solutions of the Russian Academy of Sciences in the Honour of Professor O. Ya. Samoilov, Moscow 2001. - p.50.

[15] B. Vijaya Pandiyan, P. Kolandaivel, and P. Deepa, "A theoretical perspective of the nature of hydrogen-bond types-the atoms in molecules approach," Molecular Physics, vol. 112, no. 12, pp. 1609-1623, 2014. - p.50.

[16] H. Büning-Pfaue, "Analysis of water in food by near infrared spectroscopy," Food Chemistry, vol. 82, no. 1, pp. 107-115, 2003. - p.50.

[17] M. Blanco and I. Villarroya, "Nir spectroscopy: a rapid-response analytical tool," TrAC Trends in Analytical Chemistry, vol. 21, no. 4, pp. 240-250, 2002. - p.50.

[18] V. S. Langford, A. J. McKinley, and T. I. Quickenden, "Temperature dependence of the visible-near-infrared absorption spectrum of liquid water," The Journal of Physical Chemistry A, vol. 105, no. 39, pp. 8916-8921, 2001. — p.50.

[19] E. Brini, C. J. Fennell, M. Fernandez-Serra, B. Hribar-Lee, M. Luksic, and K. A. Dill, "How water's properties are encoded in its molecular structure and energies," Chemical reviews, vol. 117, no. 19, pp. 12385-12414, 2017. - p.50.

[20] R. Röttgers, D. McKee, and C. Utschig, "Temperature and salinity correction coefficients for light absorption by water in the visible to infrared spectral region," Optics express, vol. 22, no. 21, pp. 25093-25108, 2014. - p.50.

[21] G. W. Steen, E. C. Fuchs, A. D. Wexler, and H. L. Offerhaus, "Identification and quantification of 16 inorganic ions in water by gaussian curve fitting of near-infrared difference absorbance spectra," Applied optics, vol. 54, no. 19, pp. 5937-5942, 2015. - p.50.

[22] Y. Marcus, "On water structure in concentrated salt solutions," Journal of solution chemistry, vol. 38, no. 5, pp. 513-516, 2009. - p.50.

[23] A. W. Omta, M. F. Kropman, S. Woutersen, and H. J. Bakker, "Negligible effect of ions on the hydrogen-bond structure in liquid water," Science, vol. 301, no. 5631, pp. 347-349, 2003. - p.

[24] D. Paschek and R. Ludwig, "Specific ion effects on water structure and dynamics beyond the first hydration shell," Angewandte Chemie International Edition, vol. 50, no. 2, pp. 352-353, 2011. - p..

[25] P. Schienbein, G. Schwaab, H. Forbert, M. Havenith, and D. Marx, "Correlations in the solute-solvent dynamics reach beyond the first hydration shell of ions," The Journal of Physical Chemistry Letters, vol. 8, no. 11, pp. 2373-2380, 2017. - p.50.

[26] Shimadzu recording spectrophotometer, UV-1800, instruction manual; System User's Guide. - p.51.

[27] M. Praprotnik, D. Janežic, and J. Mavri, "Temperature dependence of water vibrational spectrum: a molecular dynamics simulation study," The Journal of Physical Chemistry A, vol. 108, no. 50, pp. 11056-11062, 2004. - p.59.

[28] A. Morel, "Optical properties of pure water and pure sea water," Optical aspects of oceanography, vol. 1, p. 22, 1974. - p.61.

[29] V. Shatalov, A. Filippov, and I. Noga, "Bubbles induced fluctuations of some properties of aqueous solutions," Biophysics, vol. 57, no. 4, pp. 421-427, 2012. - p.61. 
[30] Q. Zhang, T. Wu, C. Chen, S. Mukamel, and W. Zhuang, "Molecular mechanism of water reorientational slowing down in concentrated ionic solutions," Proceedings of the National Academy of Sciences, vol. 114, no. 38, pp. 10023-10028, 2017. - p.61. 
Optofluidic chip underneath the microscope. 


\section{CHAPTER}

\section{Optofluidic chip - designs and fabrication I}

66 If I had an hour to solve a problem and my life depended on it. I would use the first 55 minutes determining the proper questions to ask.

supposedly Albert Einstein,

Part of subsection 4.2 has been accepted as G. W. Steen, A. D. Wexler, H. L. Offerhaus. "Optofluidic interferometry chip designs of differential NIR absorbance based sensors for identification and quantification of electrolytes". OSA. 2016. 


\subsection{Introduction}

The production cost of a photonic integrated circuit (PIC) can be reduced by distributing the cost of a wafer mask by multiple users. In the eighties, the semiconductor industry already used multi-project wafer (MPW) runs to prototype products, verify standard building blocks and characterize devices too expensive to fabricate in a dedicated wafer run. Most foundries in the optical industry follow the IC roadmap to reduce cost and to speed up the development of the PICs by offering MPW runs. [1, 2] Still, dedicated fabrication runs are required when the design restrictions of an MPW run cannot be followed. Here it was essential to restrict the layer thickness of the $\mathrm{Si}_{3} \mathrm{~N}_{4}$ to achieve single mode operation of the sensors in the NIR region of $0.94 \mu \mathrm{m}$ to $1.04 \mu \mathrm{m}$. Therefore, the designed optofluidic chips were produced in two dedicated wafer runs.

Unfortunately, the optofluidic chips in the first dedicated run did not show the designed single mode operation. Moreover, substantial scatter points were observed at the interface of the sensing windows. The effort spent to understand the physical origin of the issues led to improved designs presented in chapter 5 . The chips in the first run were designed with a higher fabrication tolerance in the thickness of the $\mathrm{Si}_{3} \mathrm{~N}_{4}$ core than tolerance in width. In fact, the fabrication precision is higher for the thickness. The improved design makes single mode operation more robust.

Section 4.2 presents the initial design considerations and the simulation work. The next section, 4.3, discusses the challenges of the chips from the first wafer run. In section 4.4 some general remarks are given and the lessons learned are discussed. Section 4.5 presents the optical throughput of the first chips, followed by concluding remarks.

\subsection{Design}

On-chip near-infrared (NIR) optical absorption measurements are the basis for our sensors. The combination of microfluidic channels and integrated optics lead to inline and online sensors capable of indicating danger in real time. Designs of integrated photonic NIR absorbance based sensors were optimized using simulations executed in Matlab and Optodesigner. The optical design considerations and simulation resulted in seven optofluidic chips mask designs.

The sensing principle is based on recording the NIR absorbance difference between pure water and electrolytes in the spectral region between $0.94 \mu \mathrm{m}$ to $1.04 \mu \mathrm{m}$. Identification and quantification can be achieved using differential NIR absorbance spectra, where the absorption of water with electrolytes is compared to that of pure water. Previous work (chapter 2 and 3) confirmed that a spectral bandwidth of $0.10 \mu \mathrm{m}$ centered at $0.99 \mu \mathrm{m}$ is a suitable wavelength region for the sensor. [3] This optical method operates on a relatively small change in differential absorbance of $\sim 2 \%$ induced by a 1 Molar change in electrolyte concentration. Therefore, addressing sensitivity is a critical limiting factor is required to make such sensors broadly applicable.

Different strategies can be used to detect small light intensities with orders of magnitude larger optical background - e.g., modulations of the small signal, interferometric suppression of the background, and amplification of the signal. [4] A relatively simple technique uses a Mach Zehnder interferometer (MZI); the desired differential signal 
can be directly detected by observing the light intensity in the destructive path of the MZI. [5] This method requires precise dispersion compensation, equal propagation losses in both arms of the MZI and broadband 50/50 power splitters. [6] The difference in absorption translates in a difference in intensity in both arms, and thus the differential intensity can be recorded in the destructive path of the MZI. The ratio between the power in the destructive path (dark fringe) and the power in the constructive path (bright fringe), called null depth $\left(\mathrm{R}_{\text {null }}\right)$, can be used to quantify the performance of the MZI $\left(R_{\text {null }}=I_{\min } / I_{\max }\right)$. [7, 8] Fitting the interferometric data to recover the differential intensity is another approach. $[9,10]$ For this method, a difference in the optical path is set between the two arms to record multiple spectral fringes.

Optimal performance necessitates dispersion compensation and an achromatic $\pi$ shift. Moreover, both mechanical and thermal instabilities will degrade the null depth. The use of optical integrated circuits is essential to the realization of the sensor. Y-junction power dividers are used to achromatically bisected input light to two sensing arms. Inverted $\mathrm{y}$-branch power splitters and adiabatic $\Xi$ couplers are utilized to recombine the light. Two output ports can be monitored in the classical interferometer. The on-chip interferometer exhibits an inverted y-junction for recombination of the bisected light, resulting in one output. The other output is the inaccessible cladding of the waveguide. Advantages of the Y-junction power splitter are the achromatic operation and the insensitivity to the fabrication process. The multimode interference coupler (MMI) can be designed to have multiple outputs, however they exhibit higher losses and have a chromatic operation. [11] Asymmetric (bidirectional) couplers have two outputs, however the chromatism needs to be carefully compensated. [12-14] $\Xi$ (also named tri) couplers can monitor the dark fringe achromatic. However, the contrast between the outer ports and middle port is chromatic. Both couplers are sensitive to manufacturing processes and do not preserve the initial phase difference of the input beams. [15] The contrast wavelength dependence of the $\Xi$ coupler can be flattened by an adiabatic design. [16] The adiabatic $\Xi$ coupler was used in multiple chip-designs and had to be designed in the spectral region of interest.

The evaluated designs vary in both sensitivity and complexity. All of the evaluated designs were based on strip waveguides prepared using the TriPleX platform (LioniX BV) supporting on-chip light propagation between $400 \mathrm{~nm}$ to $2350 \mathrm{~nm}$. [1] A silicon nitride $\left(\mathrm{Si}_{3} \mathrm{~N}_{4}\right)$ core is covered with a silicon oxide $\left(\mathrm{SiO}_{2}\right)$ cladding and is henceforward called the 'buried structure'. As sensing of reference fluid (water) and sample fluid (electrolyte) is required, fluidic windows are etched through the $\mathrm{SiO}_{2}$ top cladding layer so that the evanescent field can be used to probe the differential absorbance. The structure with the etch removal of the top cladding layer is henceforward called the 'sensing structure'.

The Optodesigner software package was used to find the optimal structure of the waveguide for highest sensitivity, lowest bend losses, and suppression of higher order modes. The Film Mode Matching (FMM) method was used to determine the sensitivity with the width $(w)$ and thickness $(t)$ of the $\mathrm{Si}_{3} \mathrm{~N}_{4}$ core as parameters, while the expected bend losses have been simulated by the Finite Difference (FD) method. [17, 18] Matlab 2014 was used to find the optimum length of the sensing windows by evaluation of the Beer-Lambert law with different losses of the waveguide. The dispersion was determined by the FMM method and used in Aspic to determine the required path length differences for the MZI and the homodyne detection design. Finally, the Beam Propagation Method was used to determine the parameters for the $\Xi$ coupler to achieve achromatic behavior. 
[19]

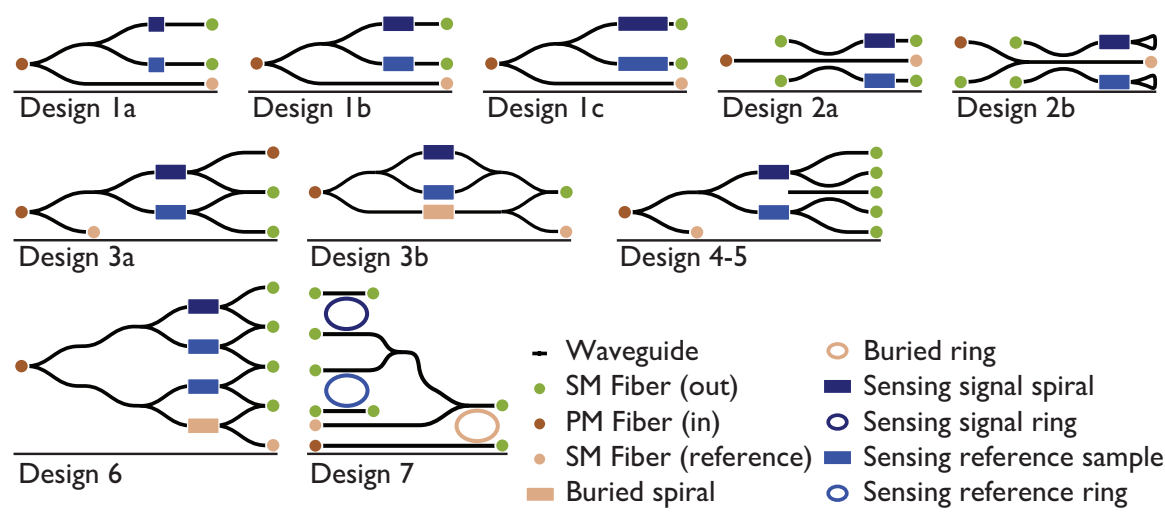

Figure 4.1: The schematic overview shows ten different optical layouts. For simplicity, the heaters are not shown. Each design contains a sensing spiral in the sample- and a reference fluidic window; however, the complexity of the optical layout varies. Design 1, design 2 and design 3 are placed on one die (chip). The differential signal is obtained by electronic subtraction. Design 2a differs only from design $1 b$ by the achromatic $\Xi$ coupler component that replaces the y-branch power splitter. The other designs feature optical subtraction schemes to obtain the differential absorbance. Design $2 \mathrm{~b}$ uses the $\Xi$ coupler to realize a Michelson interferometer on-chip. Design 3a is a simple Mach Zehnder Interferometer (MZI). Before recombining the two paths part of the light is split off for references purposes. Design $3 \mathrm{~b}$ is a homodyne setup: the output of an MZI is combined with a local oscillator, again a y-junction power divider is used to characterize part of the intensity of the local oscillator. Design 4 and 5 both contain an MZI that recombines, by use of a $\Xi$ coupler, the optical paths with a path length difference of $0 \mu \mathrm{m}$ and $280.6 \mu \mathrm{m}$, respectively. Design 6 features an internal calibrator to compensate the signal for drift caused by environmental changes, e.g., temperature. The output of the MZI without any external perturbation can be used to correct the output of the other MZI (used for sensing). Design 7 features a Vernier setup: two microring resonators (MRR) are coupled to achieve a free spectral range (FSR) comparable to a single MMR.

The simplified designs are schematically presented in figure 4.1. Additional y-branch power splitters were used in the seven designs to record fluence fluctuations and the coupling losses between the fiber array and optofluidic chip. The designs were placed on a wafer mask as shown in figure 4.2. Design 1 to 5 are copied three times on the wafer design, and design 6 and 7 are placed twice on the mask. The minimum distance between the waveguides $50 \mu \mathrm{m}$ to minimize crosstalk.

Polarizing maintaining (PM) fibers are used to guide light from the source to the input ports of the optofluidic chip, and single mode (SM) fibers are used to guide the light from the output ports to the detectors (figure 4.3). In a stable environment, the SM fibers could also be used as input channels, but an optical table would be required to minimize stress-induced polarization changes that would influence the sensor stability. [20] By coupling light into the SM fibers (normally output ports), the functionality of the chips is extended.

The three sub designs 1a, 1b, and 1c are placed on one die (chip) as shown in figure 4.4. The sub designs have different lengths for the sensing windows: $22.727 \mathrm{~mm}$ (design 1a), $68.182 \mathrm{~mm}$ (design 1b) and $113.636 \mathrm{~mm}$ (design 1c). The desired signal can be deter- 


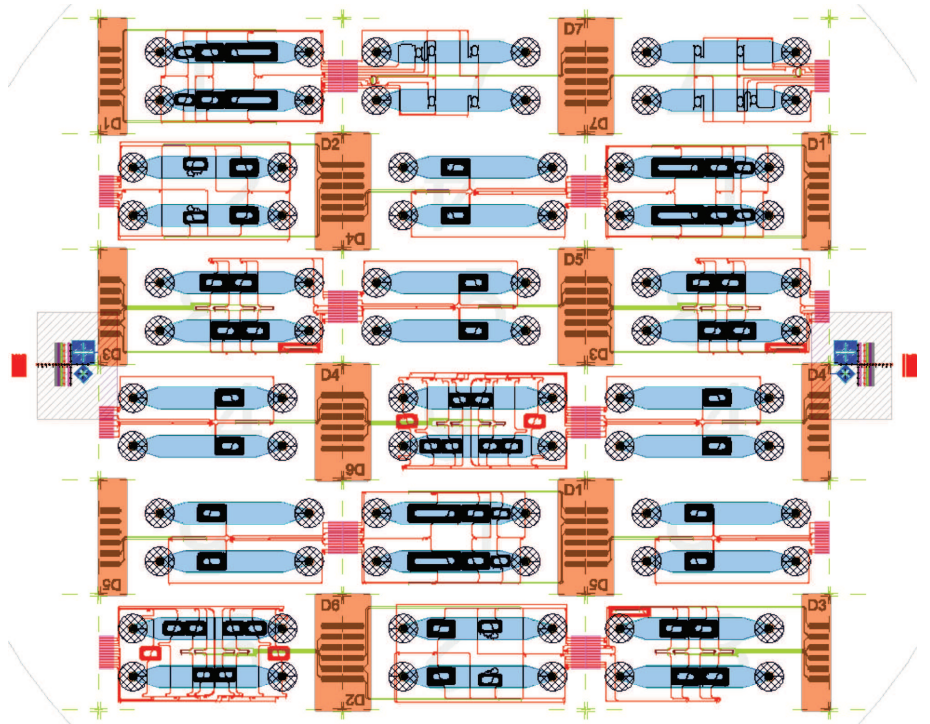

Figure 4.2: Mask design used in the first run. The blue compartment can be flushed with water or an electrolyte. The pink indicate the positions where fiber arrays should be bonded to the chips. The green lines mark areas for gold deposition. The deposition is used to connect the heaters and for chip identification. The chips are marked with gold deposition D1, D2, D3, D4, D5, D6 and D7, where the D stands for design.

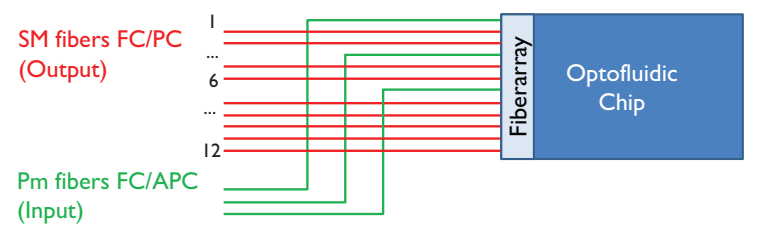

Figure 4.3: The optofluidic chips were designed to be connected to a standardized fiber array (3 PM fibers (FC/APC, PM780-HP) and 9 SM (FC/PC, 980-HP) fibers) to reduce cost. The in- and output fibers are numbered 1 to 12 , where the input channels are numbered 1,4 and 7 .

mined after electronically subtraction of the two outputs. When the in- and outputs fibers are reversed, design 1 will function as an MZI, where the phase and intensity relation between two arms can be controlled in a free space setup to achieve deeper nulling. Propagation losses of $\sim 0.5 \mathrm{~dB} \mathrm{~cm}^{-1}$ were assumed. Therefore, a sensing length of $68.182 \mathrm{~mm}$ was chosen in the other design to maximize the signal strength.

The second mask design contains two optical layouts. The adiabatic coupler in design $2 \mathrm{~b}$ (Fig. 4.5, left part of the mask design) splits the propagating light into three paths. The coupled light in the outer waveguides can sense the reference- and sample fluid, while the light in the middle waveguide can be monitored for reference purposes. A free-space mirror was mimicked on-chip by guiding the light from one output of the $\mathrm{y}$-branch power splitter into the other output. These mirrors are used to create a Michelson interferometer design: light propagating through the sensing paths were mirrored. A 


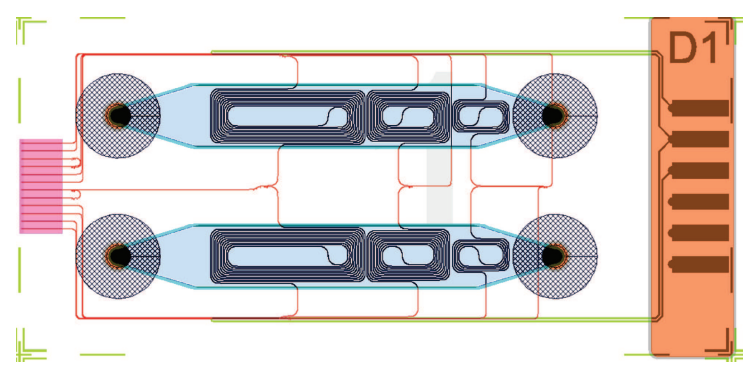

Figure 4.4: Mask design of chip layout D1. Depending on which input is used, light is coupled to a different spiral set. All 12 channels are used on this chip. Light coupled to channel 1, 4 and 7 can be monitored by channel 13,4 and 8 , respectively. The input light is guided to the smallest $(22.727 \mathrm{~mm})$, medium $(68.182 \mathrm{~mm})$ and large $(113.636 \mathrm{~mm})$ sized spiral set, respectively. The light absorption can be measured using channel 2 and 12, 5 and 11, and 6 and 9 for the three input channels 1,4 and 7 , respectively.

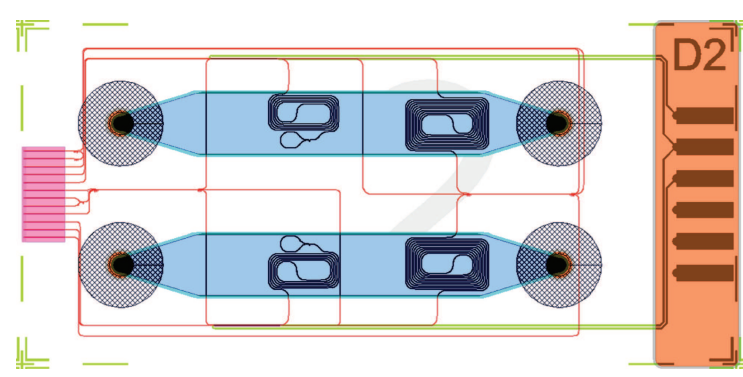

Figure 4.5: Mask design of chip layout D2. On the left side, a layout (design 2b) of a Michelson interferometer can be seen. A $\Xi$ coupler is used to split the light into three paths. The outer paths are guided to a sensing spiral of $3.4 \mathrm{~cm}$ ending with a mirror. The middle path (reference) can be monitored at Output 10 . The right side of the mask design shown a layout (design 2a) similar to design $1 \mathrm{~b}$, where the splitting of light is achieved with a $\Xi$ coupler instead of a y-junction power divider.

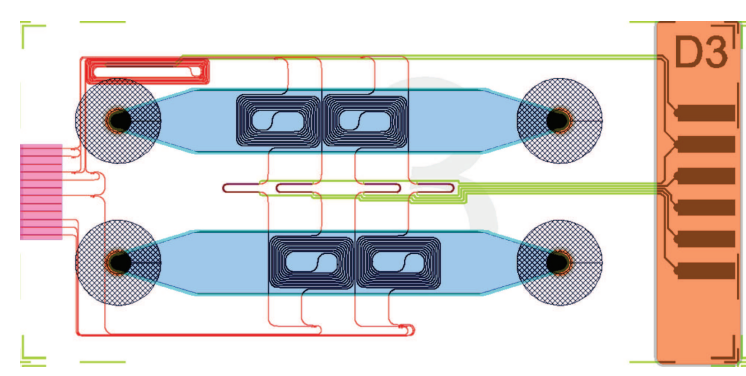

Figure 4.6: Mask design of chip layout D3a and D3b. On the left side, a layout of a homodyne interferometer can be seen. A Y-junction is used to monitor the output of the MZI that is combined with the local oscillator. On the right side, a simple MZI layout is realized. Light from both sensings arms are first split before recombination. 
path length difference of $280.6 \mu \mathrm{m}(2 \mathrm{x} 140.3 \mu \mathrm{m})$ between the two sensing paths causes 50 constructive and destructive fringes in the $100 \mathrm{~nm}$ broadband output. These fringes can be dynamically shifted by using on-chip heating elements to vary the optical path difference. Design 2a (Fig. 4.5, right part of the mask design) uses another $\Xi$ coupler to split light for sensing the two fluids. The other parameters are similar to design $1 \mathrm{~b}$. Output 2 can be monitored for reference purposes. The middle output (output 5 ) of the $\Xi$ coupler can alternatively be used for reference purposes. Design $2 \mathrm{a}$ becomes an MZI when the light is coupled to channel 4 and 12. After propagating through the spiral sets, the light is recombined by the $\Xi$ coupler. The functionality of the $\Xi$ coupler can be analyzed by coupling light to channel 5 . The $\Xi$ coupler of design $2 \mathrm{~b}$ can be analyzed by coupling light to channel 11.

The first design of the third die (Design 3a, Fig. 4.6) recombines, using a y-branch power splitter, the output of the $68.182 \mathrm{~mm}$ sensing spirals. A path length difference of $280.6 \mu \mathrm{m}$ (in the buried structure) is realized between both arms to produce 50 spectral fringes. Design 3b holds a homodyne layout: part of the reference channel is recombined with the output of the MZI with a path length difference of $400 \mu \mathrm{m}$, leading to 10 spectral beats.

The fourth optical layout uses an adiabatic $\Xi$ coupler to recombine the output of the sensing waveguides without path length difference. The light destructively interferes in the middle waveguide. The fourth and fifth design differ only by the path length difference set between the sensing arms: $0 \mu \mathrm{m}$ and $280.6 \mu \mathrm{m}$, respectively. Light from channel 1 is split by a y-junction divider. After the spiral set, the light is recombined in a $\Xi$ coupler. Heaters can affect the optical path difference between the two paths of the MZI.

The sixth design was developed to study the feasibility of online compensation. Environmental changes (e.g., temperature and pressure) can change the dispersion difference between the interferometric arms, affecting the signal. One MZI is used as an internal reference: the two arms of the MZI are only sensitive to the reference fluid to realize sensitivity to only environmental perturbations. Another MZI has one arm sensitive to the reference fluid and the other is sensitive to the sample fluid. The signal from the first reference MZI can be used in the second MZI to correct for environmental changes. Design 6 is placed twice on the die. The top fluidic window is designed to contain water (the reference fluid) and the bottom fluidic window is designed to contain the electrolyte (the sample fluid).

The last design (7) uses ring oscillators for sensing. The bend losses would be too high to have only one ring resonator to achieved 50 fringes in the $100 \mathrm{~nm}$ bandwidth. Therefore a Vernier layout was used to obtain acceptable bend losses, while the effective FSR is comparable to a single small ring resonator. [21]

\subsubsection{Simulation}

The sensitivity of the TE and TM modes for both structures were determined. The used method is described in section 5.2. Figure 4.7 shows the sensitivity as a function of the geometric properties of the core of the sensing structure for the $\mathrm{TE}_{0}, \mathrm{TE}_{1}$ and $\mathrm{TM}_{0}$ mode space. The relationship between the sensitivity and size of the $\mathrm{Si}_{3} \mathrm{~N}_{4}$ core is shown in figure 4.7 for three wavelengths that cover the spectral region of interest: $\lambda=0.94 \mu \mathrm{m}$, 


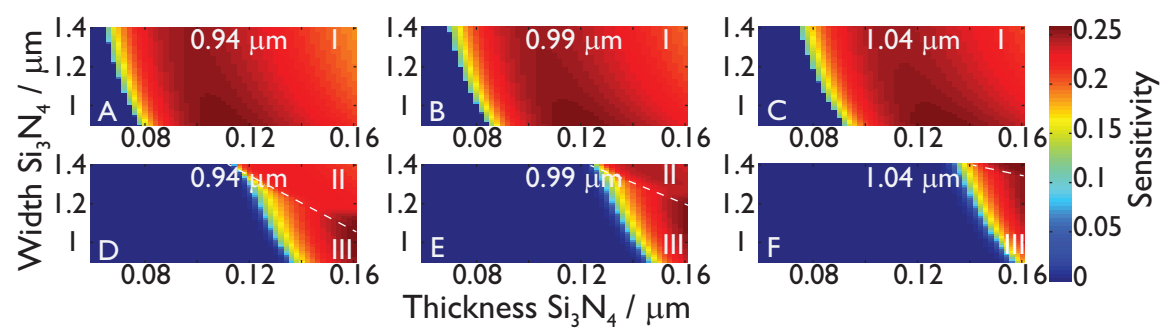

Figure 4.7: Sensitivity simulation results of $\mathrm{TE}_{0}, \mathrm{TE}_{1}$ and $\mathrm{TM}_{0}$ mode for the sensing structure as a function of the size of the $\mathrm{Si}_{3} \mathrm{~N}_{4}$ core and three wavelengths: D and G; $0.94 \mu \mathrm{m}, \mathrm{E}$ and $\mathrm{H} ; 0.99 \mu \mathrm{m}$ and $\mathrm{F}$ and $\mathrm{I} ; 1.04 \mu \mathrm{m}$. D, E and $\mathrm{F}$ show the $\mathrm{TE}_{1}$ mode. $\mathrm{G}, \mathrm{H}$ and $\mathrm{I}$ show a combination of $\mathrm{TE}_{1}$ mode and $\mathrm{TM}_{0}$ mode. The dash lines show the cut-off values for the $\mathrm{TE}_{1}$ mode (II).

\section{$0.99 \mu \mathrm{m}$ and $1.04 \mu \mathrm{m}$.}

The propagation loss due to $\mathrm{SiO}_{2}$ and $\mathrm{Si}_{3} \mathrm{~N}_{4}$ was set to zero in the simulation FD method to only model bending losses. However, additional loss due to mode shifting into the fluid by bending was also considered. The bend losses of the $\mathrm{TM}_{0}$ mode for radii smaller than $1000 \mu \mathrm{m}$ were too high $\left(>0.1 \mathrm{~dB} \mathrm{~cm}^{-1}\right)$ for $\mathrm{Si}_{3} \mathrm{~N}_{4}$ waveguides $0.15 \mu \mathrm{m}$ thick and $1.5 \mu \mathrm{m}$ wide. Both thicker and wider $\mathrm{Si}_{3} \mathrm{~N}_{4}$ would lead to lower bend losses. However, this would also allow the propagation of $\mathrm{TM}_{1}$ modes. A waveguide of $0.115 \mu \mathrm{m}$ thick and $1.0 \mu \mathrm{m}$ wide will propagate single mode $\left(\mathrm{TE}_{0}\right)$ with bend losses of $<0.001 \mathrm{~dB} \mathrm{~cm}^{-1}$ for a bend radius of $105 \mu \mathrm{m}$ and $250 \mu \mathrm{m}$ for the buried structure and the sensing structure, respectively. The losses in a straight waveguide are expected to scale as $\left(\lambda_{0} / \lambda_{1}\right)^{4}$ with the propagating wavelength. A loss of $0.5 \mathrm{~dB} \mathrm{~cm}^{-1}$ was assumed for $\lambda_{0}=1 \mu \mathrm{m}$; thus the expected losses are $0.64 \mathrm{~dB} \mathrm{~cm}^{-1}, 0.52 \mathrm{~dB} \mathrm{~cm}^{-1}$ and $0.43 \mathrm{~dB} \mathrm{~cm}^{-1}$ for the 'buried' waveguides and $0.07 \mathrm{~dB} \mathrm{~cm}^{-1}, 0.44 \mathrm{~dB} \mathrm{~cm}^{-1}$ and $0.19 \mathrm{~dB} \mathrm{~cm}^{-1}$ for the 'sensing' waveguides for wavelengths $0.94 \mu \mathrm{m}, 0.99 \mu \mathrm{m}$ and $1.04 \mu \mathrm{m}$, respectively.

After fabrication, the optofluidic chips were diced from the wafers. The criteria for choosing the best chip of design one and three are based on the highest output and best contrast of the interference fringes, respectively. The packaging of the two chips has been realized by LioniX using a setup with two translation stages. Light propagation of the SLD has been observed by capturing the scattered light with a USB microscope in a dedicated setup within the LioniX complex.

\subsection{Tools to investigate optofluidic chips}

The design of the cross-section of waveguides balances sensitivity and single mode $\left(\mathrm{TE}_{0}\right)$ operation over a bandwidth of $0.94 \mu \mathrm{m}$ to $1.04 \mu \mathrm{m}$. The waveguide allows only $\left(\mathrm{TE}_{0}\right)$ mode propagation with water, or a medium with similar refractive index, in the sensing windows. Without a liquid in the sensing windows, strong scattering points were observed at the interface between buried- and sensing waveguides as expected. An example is shown in figure 4.8 .

The observed scatter points were seen to reduce in intensity but remained present, when water was flushed through the sensing window. The scattering points also remained 


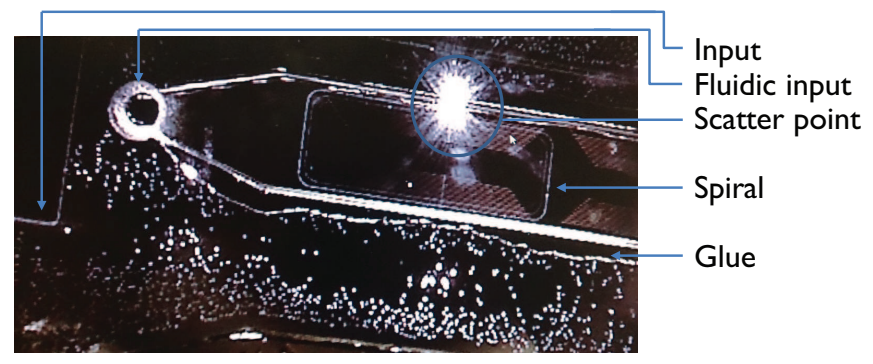

Figure 4.8: Microscopic image taken from the optofluidic chip with design D1c (long spiral length).

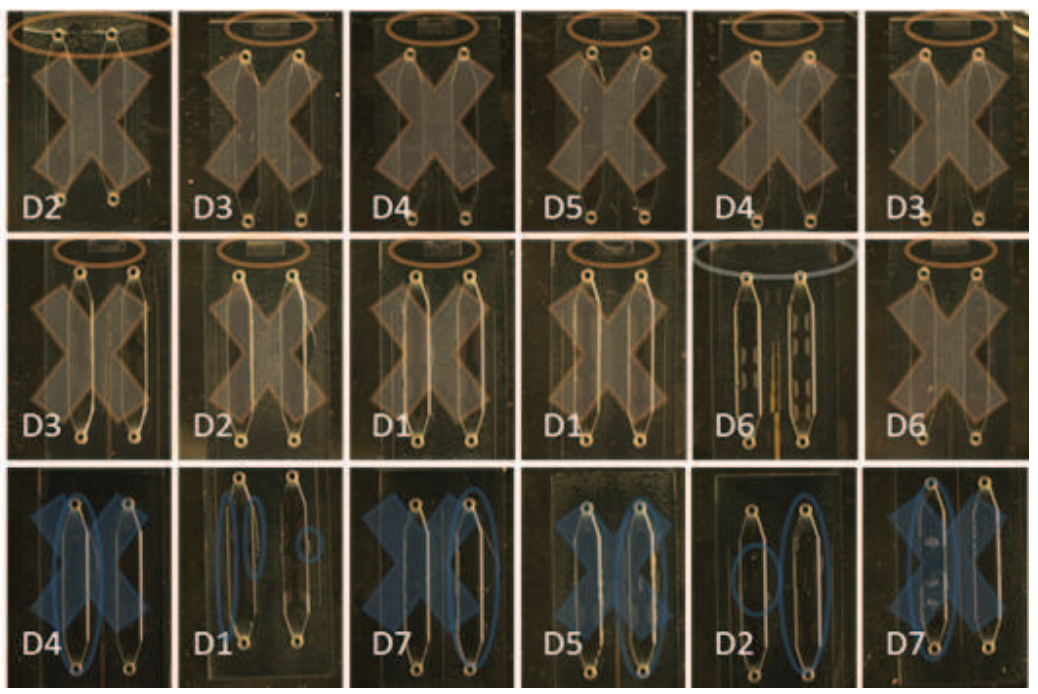

Figure 4.9: Microscopic images of the optofluidic sensor diced from wafer 3133. Problems with the coupling facet are marked with orange-brown crosses. Blue crosses indicated chips with problems with the cladding/glue.

after the fluidic windows were flushed with decane that has a refractive index close to the $\mathrm{SiO}_{2}$ cladding. Similar scatter points were observed in most chips at the interfaces of the 'buried' to 'sensing' waveguide.

The polarization of the input light was rotated to investigate the high losses. By rotating the interconnector, the propagating length was observed to increase by changing the polarization of the light by 90 degrees. This observation did not align with the simulations of the cross-section of the waveguides. The chips were designed to be single mode $\left(\mathrm{TE}_{0}\right)$, and the TM modes should not be confined by the waveguides. The scattering at the scatter points remained (though in much lower amounts).

Further characterization was performed using a dedicated setup in Wetsus, consisting of two translation stages (manual 3 -axis and manual 6 -axis). The resolution of $1.5 \mu \mathrm{m}$ offered the required precision to align the fiber array and the optofluidic chips. Some 


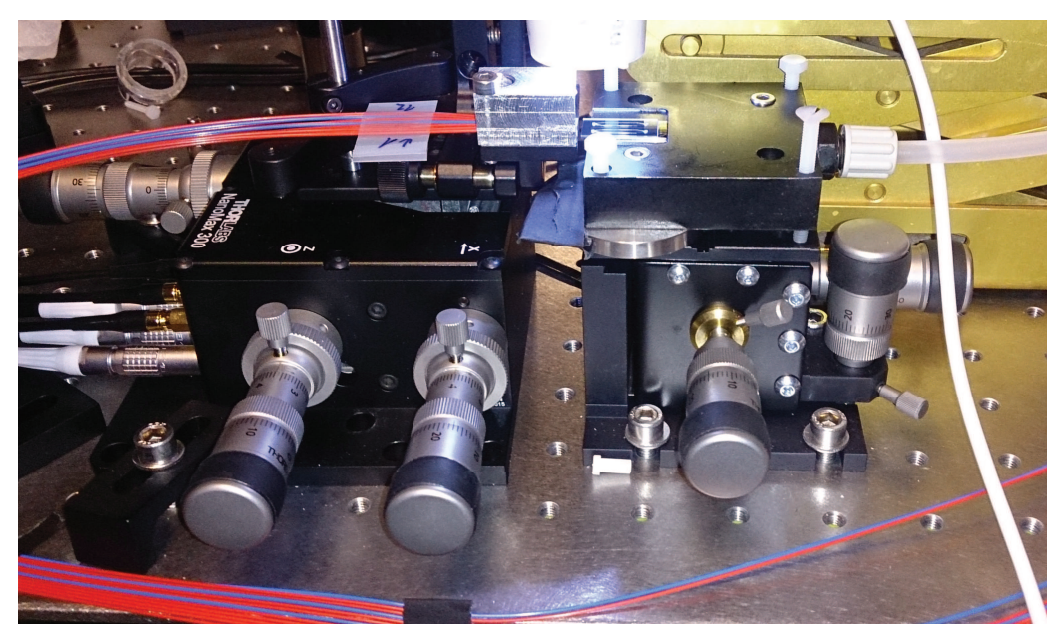

Figure 4.10: The setup used to inspect the optofluidic chips. A fiber array is positioned on the left translation stage, and an optofluidic chip is positioned on the right translation stage. The microscope can be positioned to either observe light coupling of the fiber and chip or light scattering from the spirals.

of the optofluidic chips exhibited strong scatter points. For many optofluidic chips light could not be coupled to the waveguides. Tapers designed by Lionix are used for coupling light from the fiber array to the chip. The area in which they are located appeared to be damaged, see figure 4.9). Appendix $\mathrm{C}$ shows the microscopic images taken from the other four wafers. Based on the microscopic images the chips were collected that had this area intact. The chip yield with intact coupling tapers was $55.6 \%$. That number was reduced due to problems with the windows to $38.9 \%$.

\subsubsection{Optical microscope}

A USB optical microscope (Conrad Benelux, Oldenzaal, NL) was mounted above the optofluidic chips (Fig. 4.10) to observe the propagation losses and bend losses and to provide a visual aid to align the coupling of light from the FA to the optofluidic chip (and vice versa). A mode without propagating losses would not be observed with this method. However, the roughness of the $\mathrm{Si}_{3} \mathrm{~N}_{4}$ core due to etching and other scattering inducing points (e.g., high contrast in refractive index) will lead to scattering losses of the propagating mode. Part of the scattered light can be collected by the microscope (200x, 9MP). The propagation losses can be determined by observation of scattered light intensity over distance. Similar, the bend losses can be estimated by comparing the scattered light intensity before and after the waveguides bends. It must be noted that the SLD also has spectral output ranging from $1040 \mathrm{~nm}$ to $1070 \mathrm{~nm}$. The intensity is low, $\sim 7 \mathrm{~dB}$ to $20 \mathrm{~dB}$ less than that of the central wavelengths. However, this light will have higher bend and propagation losses and will contribute to the light scattering observed with the microscope.

The resolution $(R)$ of an optical microscope is limited to the diffraction limit: $R=\lambda / 2$ 
NA, where NA stands for the numerical aperture. Hence, the NIR light collected by microscope can resolve the spirals and is suitable to assist initial alignment of fiber array and an optofluidic chip for efficient light coupling. The optofluidic chip with no apparent defect in the top cladding, where further inspected by coupling light to the chip.

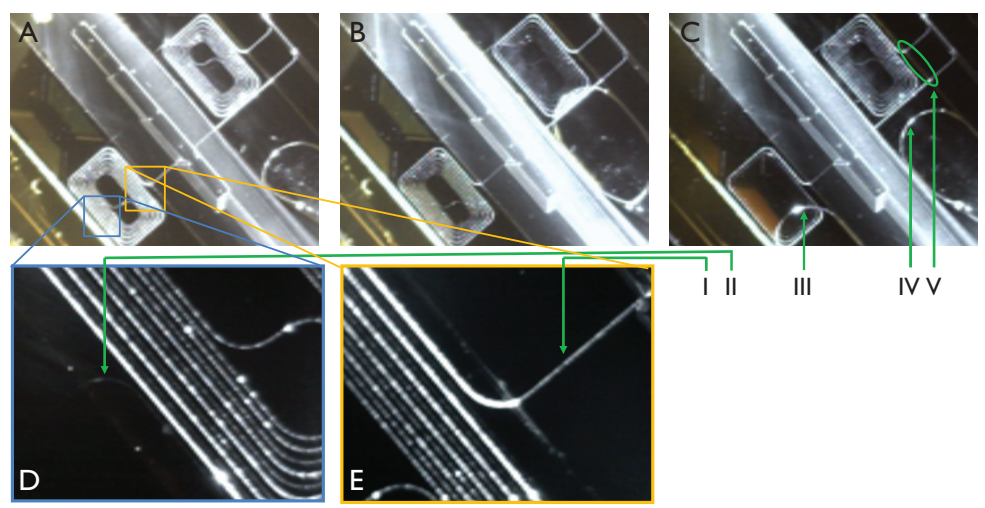

Figure 4.11: Microscopic images taken of the optofluidic chip, after the broadband light is coupled to the chip. A) The continuous decrease in scattered light can be observed. A) An air bubble approaches the top sensing spiral. B) An air bubble is positioned on the top sensing spiral. The scattering intensity is reduced in both spirals. C) Another air bubble is located on the bottom sensing spiral. D and E) An inset showing the decrease of light scattered for the propagated distances in the bottom spiral. The line arrows I, II, III, IV, V indicate light scattering of light propagating through the buried structure before entering the sensing spiral, light scattering of light propagating at the end of the sensing spiral, air bubble in the bottom fluidic window, air bubble in the top fluidic window and the interface between buried/sensing structure, respectively.

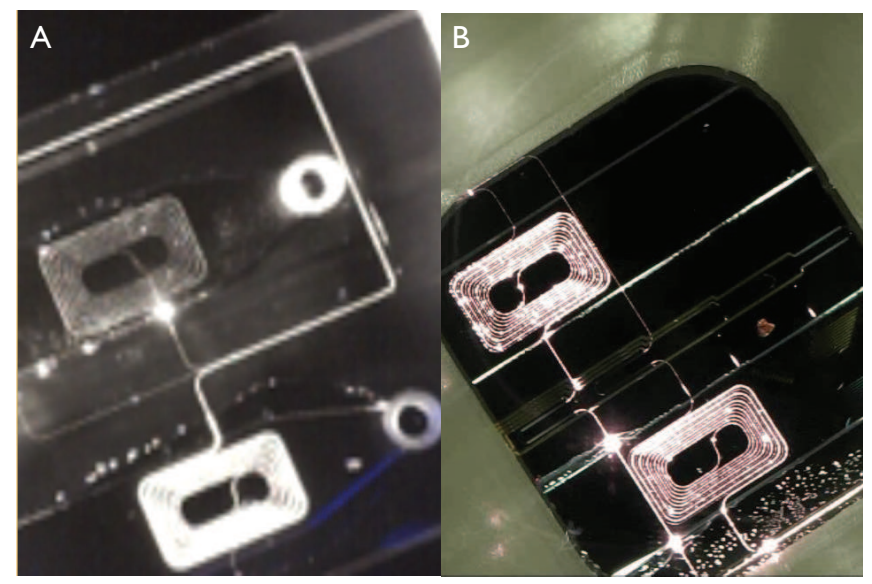

Figure 4.12: Scatter points observed by a microscope with two different two optofluidic chips. A) TM mode propagation in the chip diced from wafer 3112, design 2. A large scatter point is recorded in the top window. B) Similarly, propagation of TE mode(s) in design 3a of a chip diced from wafer 3089. The image is recorded with a higher resolution. 


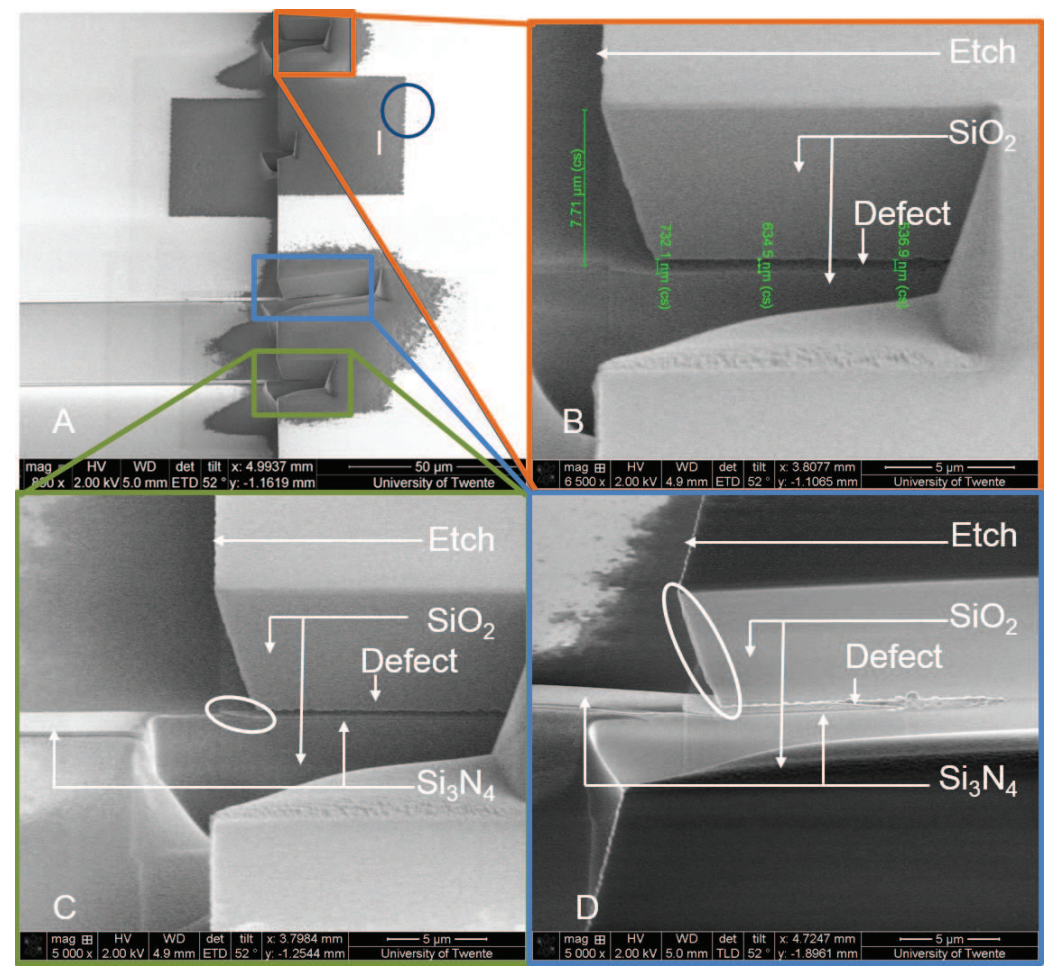

Figure 4.13: Images taken with a scanning electron microscope after milling with the focus ion beam (FIB). A) shows an overview of the different milling positions at the interface. The gold coating is removed due to the FIB. B) The height of the $\mathrm{SiO}_{2}$ layer and the defect is marked: $7.71 \mu \mathrm{m}, 0.73 \mu \mathrm{m}, 0.63 \mu \mathrm{m}$ and $0.54 \mu \mathrm{m}$. C) Effect of the Gaussian profile of the FIB and the angle of the etched $\mathrm{SiO}_{2}$ is observed at the circle. D) The backscattered electron image shows more details of the defect.

Figure 4.11 shows the decrease of scattered light intensity over distances. Light is guided over the $68.182 \mathrm{~mm}$ spirals with water in the sensing windows. This chip exhibit an intact top cladding as can be seen in the interfaces between buried/sensing structures in figure $4.11 \mathrm{C}$. The effect of air as top cladding can be seen by comparing figure $4.11 \mathrm{~A}$ and $\mathrm{B}$. The reduced scattered intensities also show an example of increased losses due to the air bubble.

It was clear that the problematic scatter points were located close to the interface between buried structure and sensing structure. Furthermore, a modulation was observed in the spectral signal that was affected by the ion concentration in the water. Multimode propagation would be the most logical reason to explain the observed behavior. A scanning electron microscope (SEM), atomic force microscope (AFM) and optical coherent tomography (OCT) were employed to investigate the physical origin of the scattering points observed in figure 4.12 and the secondary modulation on the spectral signal . 

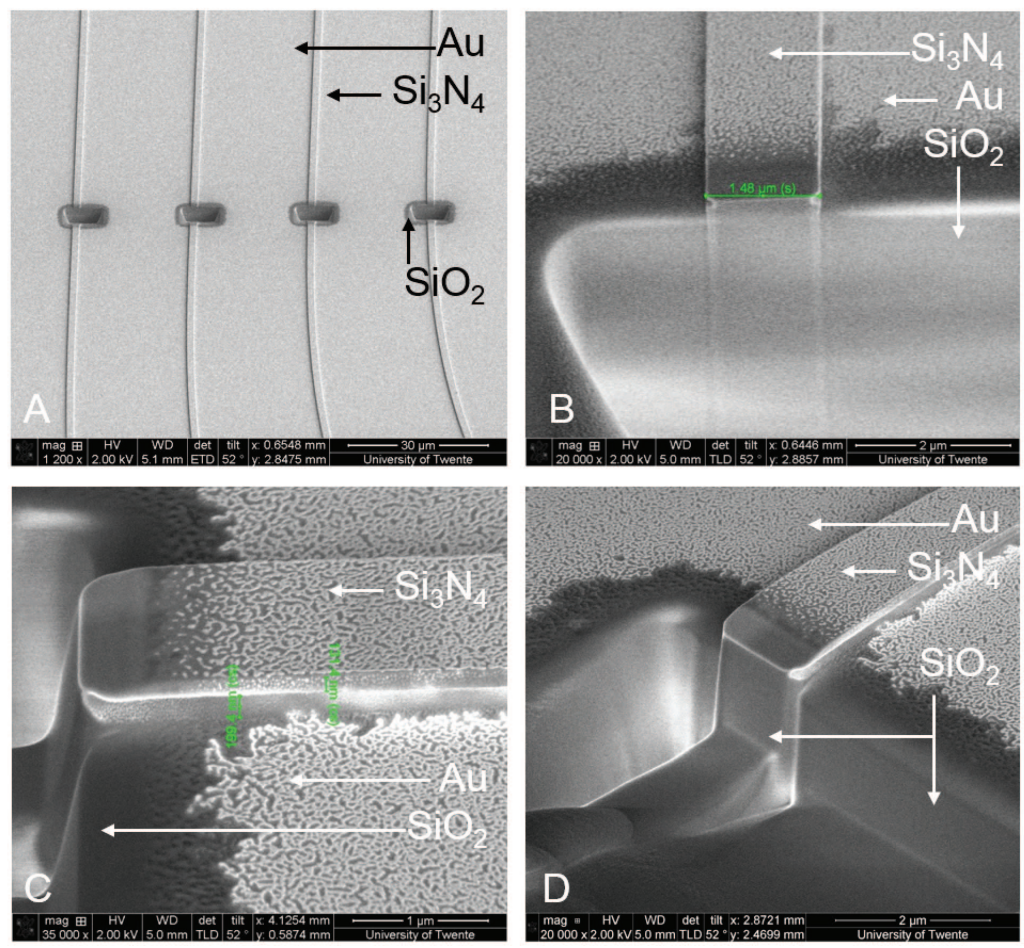

Figure 4.14: SEM images of the sensing spiral. A) Multiple holes are milled with a FIB to inspect the cross-section of the sensing waveguides. B, C and D) An overetch is observed for the crosssection of the sensing waveguides. Furthermore the width and thickness of the Si3N4 core is determined to be $1.5 \mu \mathrm{m}$ and $\sim 130 \mathrm{~nm}$.

\subsubsection{Scanning electron microscope}

Higher magnification is required to observe the cross-section of the structure. An SEM easily achieves resolution better than $25 \mathrm{~nm}$. An SEM generates a beam of electrons and uses a high potential $(\sim \mathrm{kV})$ to accelerate electron beam through a high vacuum. Secondary electrons are generated at the surface of the sample. A secondary electron image (SEI) provides topographic information of the sample. A backscattered electron image (BEI) provides information on the composition of the sample since the intensity of backscattered electrons is dependent on the atomic number of the specimen.

Higher potential results in deeper travel range of primary electrons and thereby the yield of secondary electrons is decreased resulting in reduced image quality of the surface, while the yield of backscattered electrons generated below the surface is increased. Moreover, a phenomenon called "charging"" can occur, where the secondary electrons do not originate from the surface but the edges of the material. This effect can be minimized by adequately coating the sample with a metal, e.g., Au-Pd, Pt or carbon-based conductors.

Figure 4.13 shows a gap between the lower cladding and top cladding (both $\mathrm{SiO}_{2}$ ). The gap was observed at every interface that was milled by a focused ion beam (FIB) 

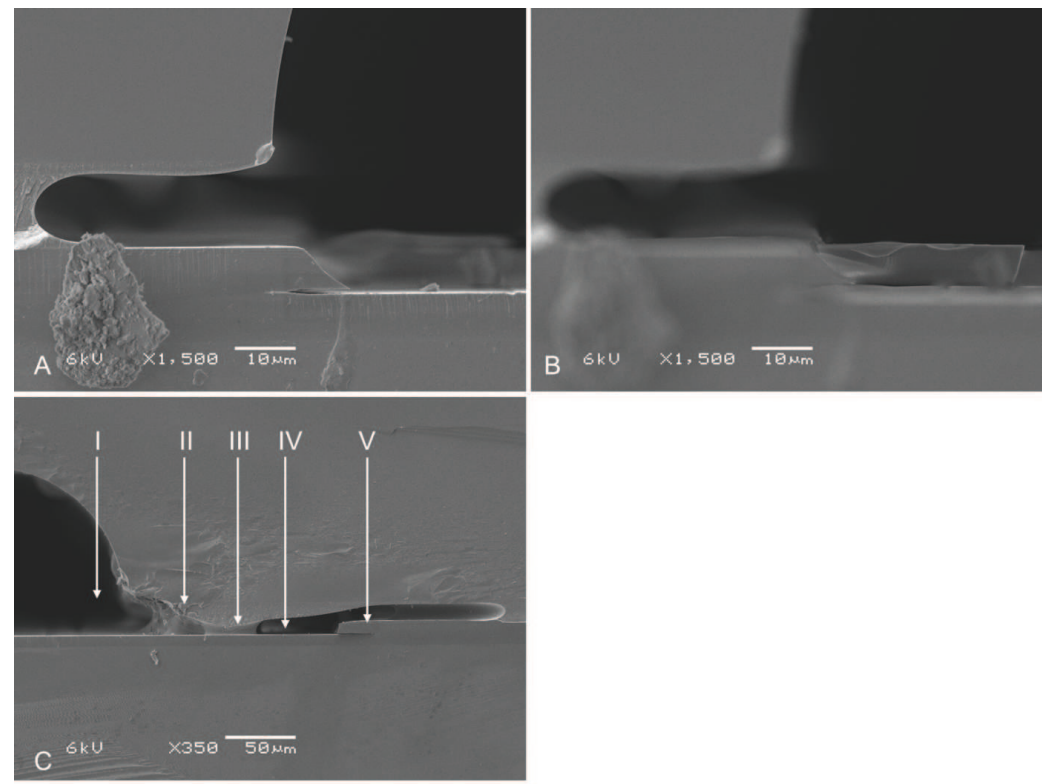

Figure 4.15: SEM images are shown of the optofluidic chip cross-section. A and B) These SEM images are taken of the left-hand side part of the sensing window with a different focus. C) The right-hand side part of the sensing window, where I is inside the fluidic window, II indicates the glue and III shows the end of the fluidic window, air is trapped in IV. V indicates the top cladding.

at multiple positions of the chip. These gaps between the top and bottom cladding are $\sim 0.5 \mu \mathrm{m}$ in height and are $\sim 15 \mu \mathrm{m}$ long. The secondary electron images A, B and C in figure 4.13 were acquired with an Everhart-Thernley detector, while the BEI was acquired by a through-lens detector.

Multiple holes were drilled in the sensing area to inspect the waveguide dimensions. In figure 4.14A the first four holes are shown (later eight more holes were drilled with shorter FIB times). While the fabricated mask should have produced $1.1 \mu \mathrm{m}$ wide waveguides, a width was measured between $1.3 \mu \mathrm{m}$ and $1.5 \mu \mathrm{m}$ by three different SEM devices (Wetsus, University of Twente). Moreover, typical trademarks of over-etched structures were observed (Fig. 4.14). The over-etching is probably also the reason for the defect seen between the top and bottom cladding.

A chip was successfully bisected which allow observation of the chip with the glass window on top and the location of the glue. Figure 4.15 shows a cross-section similar to recorded images shown in figure 4.13. It became clear with the cross-section including the window alignment that part of the $\mathrm{SiO}_{2}$ top cladding is broken off. The alignment of the window left, and right is recorded. The alignment on the left is proper (the window could be moved $\sim 30 \mu \mathrm{m}$ to the right) for the 'sensing' waveguides will sense the water. However, the alignment of the right part of the window with the etched area is $\sim 125 \mu \mathrm{m}$ too far on the left. The effect can be seen in figure 4.15, where (I) shows the sensing window under the glass window, (II) the glue, (III) the place where the sensing window stops, (IV) the location of the expected air bubble and (V) the location of the waveguide to become "sensing". A 50 $\mu$ m propagation of light can be expected with air as top 


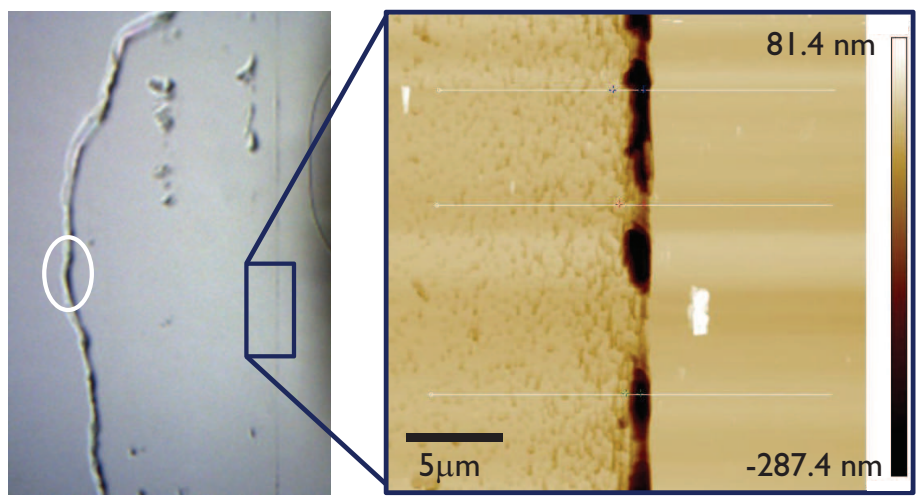

Figure 4.16: A microscopic image taken of the interface is shown at the left-hand side of the figure. The line in the white ellipse was first though to be glue deposition. The blue square shows the area of the chip where AFM data was collected. The AFM tip was positioned close to the expected interface between buried and sensing structure (height difference of $8 \mu \mathrm{m}$ ), instead, a smooth surface was observed.

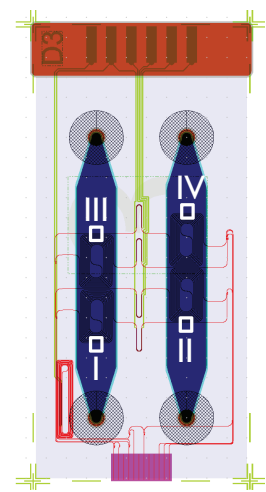

A

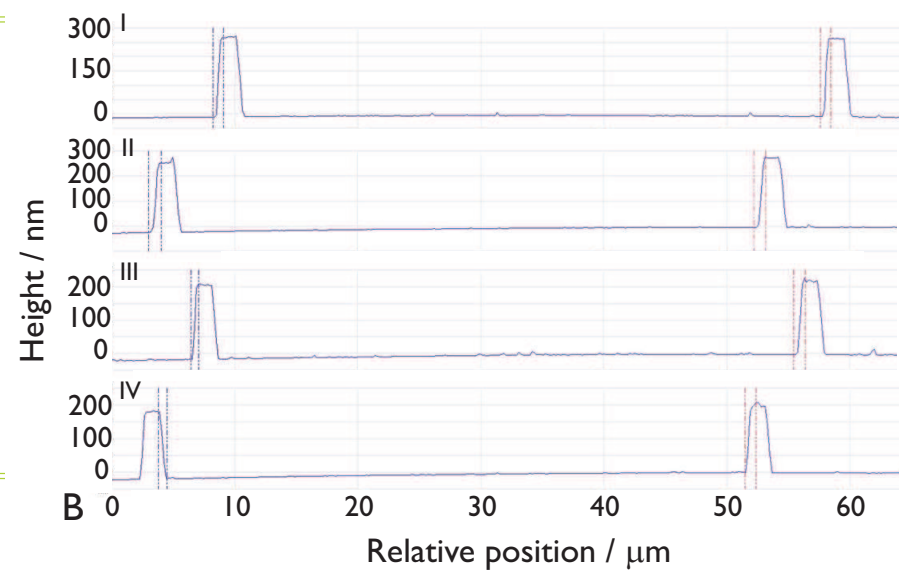

Figure 4.17: The schematic overview of the positions on the chip measured with the AFM is shown in A. The AFM images are recorded at four locations: Left of the top sensing window (I), left of the bottom sensing window (II), right of the top sensing window (III) and of the right of the bottom sensing window (IV). B) The AFM recordings of the height at the relative positions. The vertical axis and the horizontal axis give the height in $\mathrm{nm}$ and the location in $\mu \mathrm{m}$, respectively.

cladding and a $\sim 20 \mu \mathrm{m}$ propagation of light with glue as top cladding due to the size of the glass window. This leads to unknown dispersion and significant additional losses.

\subsubsection{Atomic force microscope}

Atomic force microscope (AFM) was used to gain structural information of the chips to improve the optical microscopic images that lack the topographical information. The line observed with the optical microscope in figure 4.16 was assumed to be a glue 


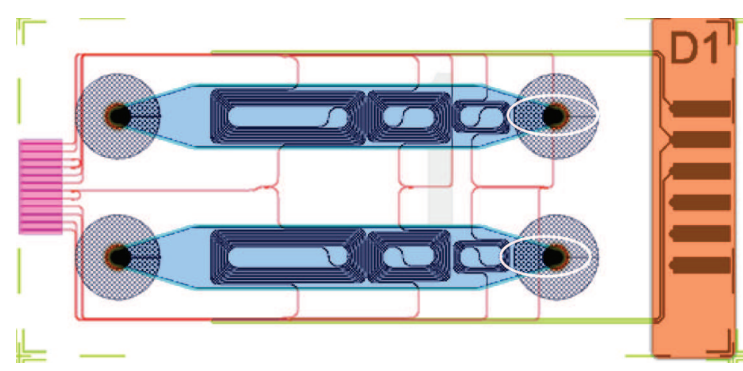

Figure 4.18: Mask design of chip design 1. The white ellipses show locations where waveguides pass underneath the fluidic channel. Extra losses are induced due to interface and propagation losses.

deposition. It became apparent after the AFM recordings that the line seen with the optical microscope is the interface between buried and sensing structure (moved outside the fluidic window area) due to breakage of the top cladding.

The difference in etch depth can be seen in figure 4.17 between the left and right locations. LioniX measured the nitride layer (by ellipsometry) of $115.4 \mathrm{~nm}$ after deposition. The thickness after etching was estimated as $114.3 \mathrm{~nm}$. The etch rate was given as $<0.5 \mathrm{~nm} / \mathrm{min}$ and $\sim 65 \mathrm{~nm} / \mathrm{min}$ for the $\mathrm{Si}_{3} \mathrm{~N}_{4}$ and $\mathrm{SiO}_{2}$, respectively. Height differences of $\sim 200 \mathrm{~nm}$ and $\sim 270 \mathrm{~nm}$ are observed, indicating an $85 \mathrm{~nm}$ and $155 \mathrm{~nm}$ overetch, respectively. This overetch depths would be expected with one, and two minutes etch time, leading to a nitride layer thickness of $114.8 \mathrm{~nm}$ and $114.2 \mathrm{~nm}$, respectively.

\subsection{Lessons learned}

The fabrication tolerance in width of the $\mathrm{Si}_{3} \mathrm{~N}_{4}$ core ensured by lithography is less than the tolerance in thickness of the $\mathrm{Si}_{3} \mathrm{~N}_{4}$ core that uses etching steps. The second set of optofluidic chips were based on the minimum width tolerance $(1.6 \mu \mathrm{m})$ to achieve single mode operation with a $\mathrm{Si}_{3} \mathrm{~N}_{4}$ thickness of $0.092 \mu \mathrm{m}$. The width of the waveguides on mask-design 2 and 4 were slightly varied in width to compensate for the fabrication precision in width.

The reference channel is used to probe fluence fluctuations of the light source. The first designs used symmetrical y-splitters to bisect the input light. Directional couplers have been designed by LioniX and used in the second designs to monitor the power fluctuations. Part of the input light is chromatically split to the reference. However, this disadvantage of wavelength dependent SNR of the recorded fluence fluctuations is outweighed by the increase of signal strength.

The first wafer run had a low yield of chips due to the improper bonding of the top $\mathrm{SiO}_{2}$ cladding. A full report is given on chips of the five wafers in Appendix C. Additional precaution was taken to prevent the breaking of the top cladding. The area of etching was reduced to only remove the top-cladding above the sensing spirals (shown in Appendix D). This precaution also reduces interface and propagation losses of light passing underneath the fluidic window without sensing purpose.

The mask layout with the different chip designs was made by spreading copies of each mask design of the chips on a wafer size mask. The mask design of some chips was 


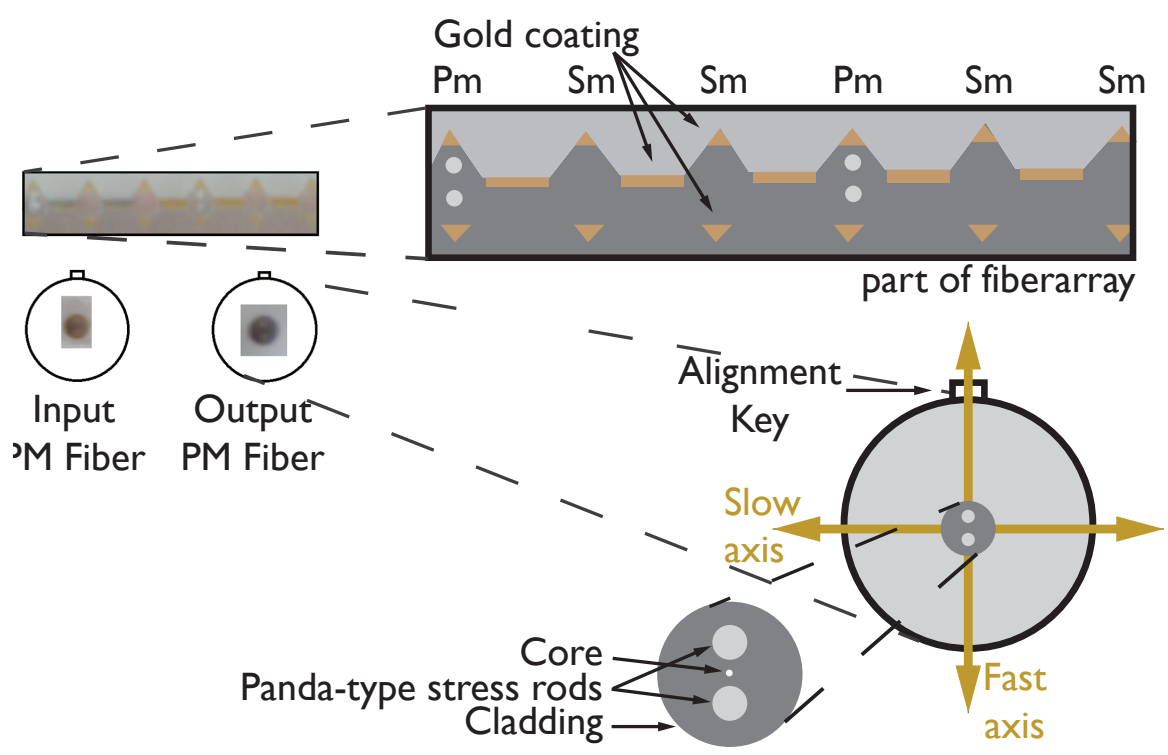

Figure 4.19: Microscopic images were taken of the fiber array and polarizing maintaining (PM) fibers. The black drawn outline of the PM fiber shows the position of the alignment key (not on scale). The insets show the schematics of the fiber array and the 'panda' style PM fibers. The second inset shows the core of the PM fiber.

flipped both vertical and horizontal to have the fiber arrays facing each other. However, the vertical flipping causes the waveguides on-chip to be misaligned with the arrangement of the fiber array. This was overlooked in the first dedicated run and was only observed when the light was coupled to the chips. The issue can be solved by flipping the FA, however, this requires a new design of the chip holder. The misalignment was avoided in the second fabrication,

The chips have been designed to propagate only the zero order TE (electrical field in the horizontal plane) mode. The light source delivers the TE mode on the slow axis, while the FA delivers it on the fast axis. The TM mode was delivered on the chip due to this configuration. The polarization was confirmed by the need to rotate the fiber with 90 degrees (setting the alignment key of the fiber horizontally) in order to obtain the minimum reflection at the Brewster angle. Also, a microscope was used to inspect the facets of the fibers and fiber array (Fig. 4.19). The microscopic images confirmed the TE mode to be vertically aligned from the fiber-array to the chips. A cross-PM fiber was inserted between the light source and the fiber array to offer the correct polarization. This fiber induced additional losses and may induce a modulation on the signal recorded from the optofluidic chip due to Fabry-Perot interference. 


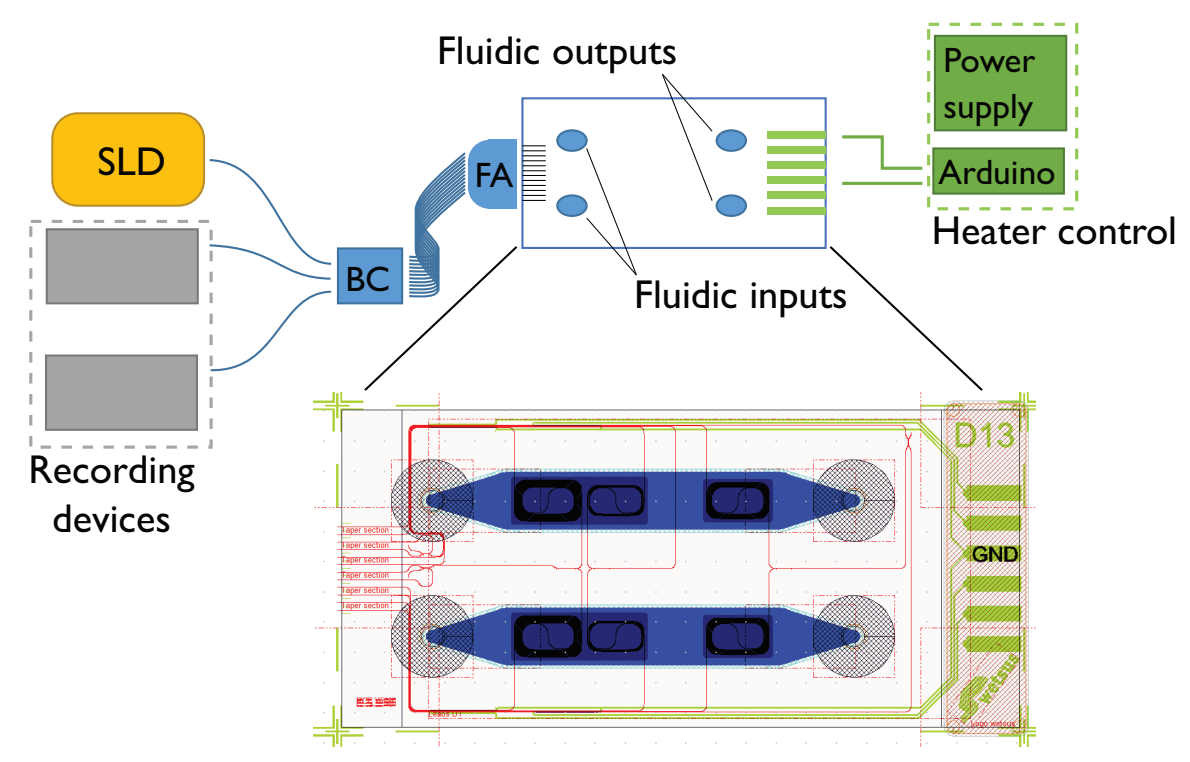

Figure 4.20: Schematic of the setup. Light from a broadband light source (SLD) is butt-coupled (BC) to a fiber array (FA) and guided to the chip. The blue color in the zoom-in shows the microfluidic channel. The top cladding is removed to enable sensing in the dark blue region. The output light is coupled back to the FA and read by the spectrometers. The green lines depict the electrical connections to the heaters.

\subsection{Optical throughput chips - first run}

\subsubsection{Materials \& Procedure}

A fiber tower was built to facilitate the experiments. It consisted of a breadboard and 12 narrow-key-slot mating sleeves. The chips were mounted on a bisected chip holder and placed on a translation stage with 6 degrees of freedom (Thorlabs Nanomax Max607/M, Newton, NJ, USA). The fiber-array was placed upon a second translation stage (Thorlabs Nanomax 300, Newton, NJ, USA). The facet of the fiber-array was butt-coupled to the chip without the aid of index matching gel. Two PM fibers (FC/APC - SMA) were coupled to the output fibers and connected to the recording devices. The output of a power meter (S150C, Thorlabs, DE) was used to optimize the alignment of the FA and chip.

A broadband light-source (BLMS-mini-521-HP,Superlum ${ }^{\mathrm{TM}}$, Carrigtwohill, Co. Cork, Ireland) provided polarized light with a $92.2 \mathrm{~nm}$ bandwidth at a central wavelength of $994.2 \mathrm{~nm}$. An $7.8 \mathrm{~mW}$ output power, a spectral ripple of $1.6 \%$, and a power drift of $<0.3 \%$ per 4 hours were specified by the manufacturer. In figure 4.20 the schematic layout of the setup is presented. A polarization maintaining (PM) fiber was used to coupled light from the super-luminescence diode (SLD) to an input fiber of the fiber-array (FA).

The ohmic resistance of the gold layer on top of the waveguides was locally increased to realize heaters on the chip. The power dissipation of a power supply (E300-0.1L, Delta 
Elektronika, Zierikzee, Nl) on the chip was regulated by a microcontroller (Arduino Uno, open-source hardware). The Arduino had a separate power supply (Alimentation Symetrique Al 890 NX, L'impulsion, Hérouville St Clair,FR) and was operated with a regular desktop computer. Two syringe pumps were used to flush the solutions through the fluidic windows. Two spectrometers (Ava Spec-ULS3648, Avantes BV, Apeldoorn, NL) with a sensitivity of 160 counts $\mathrm{mW}^{-1} \mathrm{~ms}^{-1}$ and a resolution of $0.6 \mathrm{~nm}$ were utilized to record the spectral transmission through the chips.

Stock solutions of $\mathrm{NaOH}(0.0613 \mathrm{M}, 0.125 \mathrm{M}, 0.25 \mathrm{M}, 0.5 \mathrm{M}$ and $1 \mathrm{M})$ were prepared to measure the optical response of the chips based on optical design 1 (electronic subtraction) and design 3 (optical subtraction). The solutions were prepared using demineralized water, precision $( \pm 0.1 \mathrm{mg})$ balance (PIONEER ${ }^{\mathrm{TM}}$, Ohaus, Florham Park, NJ, USA) and reagent or higher grade salts (Sigma Aldrich, St Louis, MO, USA). All solutions were flushed through $0.45 \mu \mathrm{m}$ filters (Millipore Millex-LCR, VWR, Amsterdam, NL) to prevent clogging of the microfluidic windows.

The outputs of several D1, and D3 chips were recorded with the power meter, and with one of the spectrometers, respectively. Chips were selected based on the lowest propagation losses, and highest fringe depth for chips with design 1 and 3 , respectively.

The influence of dissolved salt on the transmission spectrum of water was determined by recording a data sets for a D1 and D3 chip. To collect these data sets the following procedure was used. First, 200 baselines were recorded after both windows were flushed with demineralized water. Then, the sample window was flushed by the sample of the solution to be measured ( 5 times $1 \mathrm{~mL}$ ). Next, 200 additional spectra were collected. This step was repeated for each sample until the highest concentration $(1 \mathrm{M})$ was reached. Finally, the sample window was flushed with distilled water and 200 spectra were recorded. the solutions were flushed through the top window and repeated for the bottom window of the optofluidic chips (using the fluidic pumps).

\subsubsection{Results}

The data collected from chip with design 1 is shown in figure 4.21. The entire bandwidth of the SLD was transmitted through the chips. The sensor shows a linear response in the spectral window of $993 \mathrm{~nm}$ to $1050 \mathrm{~nm}$ for concentrations of the $\mathrm{NaOH}$ solution higher than $\sim 0.25 \mathrm{M}$. However, the sensor appears to be contaminated by the solution: the sensor does not return to its initial values after flushing with water. The $\mathrm{Si}_{3} \mathrm{~N}_{4}$ core is in direct contact with the fluid. Oxidation of the $\mathrm{Si}_{3} \mathrm{~N}_{4}$ core and sodium ion migration to the $\mathrm{Si}_{3} \mathrm{~N}_{4}$ core are therefore possible explanations. [22, 23]

Fringes are visible in the spectral region of $935 \mathrm{~nm}$ to $990 \mathrm{~nm}$. The spectral shape of these fringes changes with the concentration of $\mathrm{NaOH}$. The wider waveguides also allow $\mathrm{TE}_{1}$ and $\mathrm{TM}_{0}$ mode to propagate. The $\mathrm{TE}_{0}, \mathrm{TE}_{1}$ and $\mathrm{TM}_{0}$ mode have different effective refractive indexes, that can explain the observed multimode behavior.

In figure 4.22 the data collected from chip with design 3 is shown. The fringe spacing of the MZI is affected by the concentration of $\mathrm{NaOH}$, showing the mode propagating through the sensing windows to be sensitive to the ion concentration. However, a second modulation is dependent on concentration as can be seen in the spectral region of $935 \mathrm{~nm}$ to $1005 \mathrm{~nm}$. The fringe spacing resulted in 16 spectral fringes in the region of $940 \mathrm{~nm}$ to $1040 \mathrm{~nm}$ with a fringe depth of $15 \mathrm{~dB}$. 


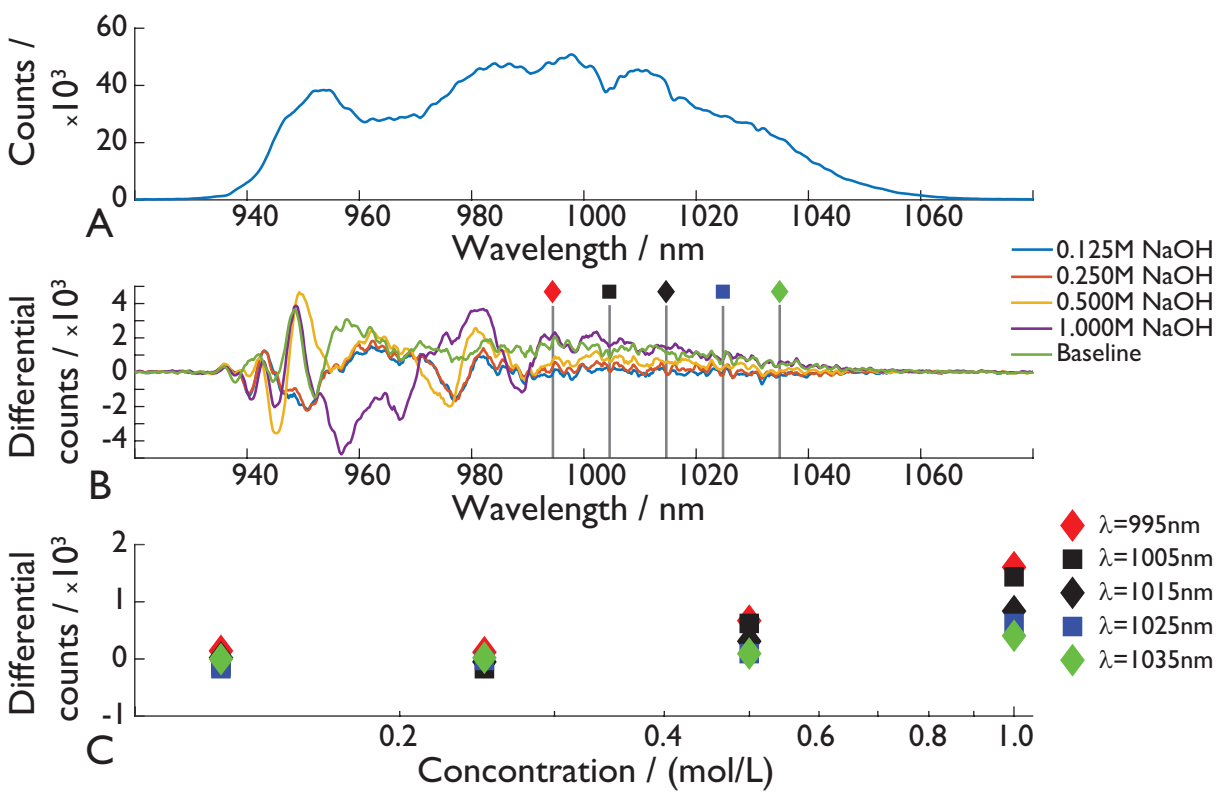

Figure 4.21: Optical recordings of the light transmitted through an optofluidic chip (design 1). A) The optical spectral throughput recorded after both fluidic windows were flushed with demineralized water (baseline). B) The spectral response difference due to the presence of ions. The baseline spectrum is subtracted from the spectra recorded for the electrolyte with different concentration. The concentration of $\mathrm{NaOH}$ increases from $0.125 \mathrm{M}$ to $1 \mathrm{M}$. C) The optical transmission difference due to the concentration of $\mathrm{NaOH}$ is plotted for five wavelengths: $995 \mathrm{~nm}, 1005 \mathrm{~nm}, 1015 \mathrm{~nm}$, $1025 \mathrm{~nm}$ and $1035 \mathrm{~nm}$. Five vertical lines are positioned at these wavelengths in B for visual aid (marked with $\diamond \mathbf{\square} \nabla ⿱$ ) ).

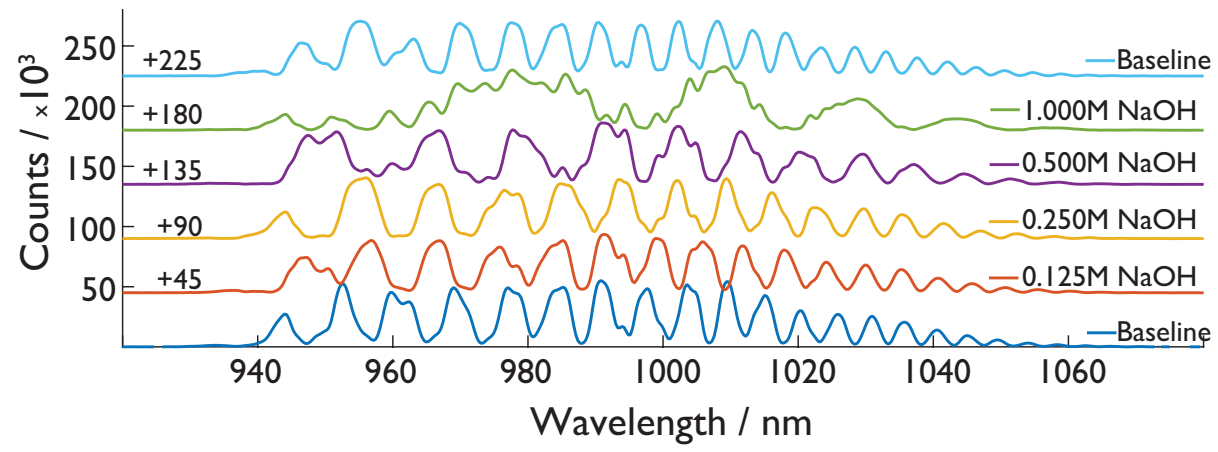

Figure 4.22: Spectral recording of an optofluidic chip with design 3 . The different measurements are stacked plotted with an increasing offset. The sensor has multimode behavior for the shorter wavelengths $(<995 \mathrm{~nm})$. The fringe spacing is dependent on the concentration of the electrolyte. 


\subsection{Conclusion}

The design considerations of optofluidic chips intended for identification and quantification of ionic content in water have been presented. The chips based on these considerations were realized by LioniX. The chip yield with intact coupling tapers (used to couple light from and to the fiber array) was $55 \%$ due to the quality of the top-cladding. That number was reduced further due to breakage of the top-cladding near the fluidic windows $\left(38.5 \%\right.$ ). The width of the $\mathrm{Si}_{3} \mathrm{~N}_{4}$ was determined (with SEM) to be $1.3 \mu \mathrm{m}$ to $1.5 \mu \mathrm{m}$, which explained the multi-mode behavior observed for the spectral region below $\sim 1 \mu \mathrm{m}$.

Many valuable learned lessons proved to be vital in the second run. The most significant improvement was the reduction of the etching area to avoid the potential breakage of the top cladding in the case of over-etching. Furthermore, the improved designs exhibit a higher width tolerance ( $200 \mathrm{~nm}$ versus $100 \mathrm{~nm}$ ) to avoid the multi-mode behavior. Even with the presented issues the optical recombination of the $6.8 \mathrm{~cm}$ sensing spirals showed a null depth close the expected null depth by considering the $0.6 \mathrm{~nm}$ resolution and optical path difference (calculated from the fringe spacing). 



\section{Bibliography}

[1] R. Heideman, A. Leinse, M. Hoekman, F. Schreuder, and F. Falke, "Triplex ${ }^{\mathrm{TM}}$ : The low loss passive photonics platform: Industrial applications through multi project wafer runs," in Photonics Conference (IPC), 2014 IEEE, pp. 224-225, IEEE, 2014. - p.70, 71 .

[2] M. K. Smit, X. Leijtens, E. Bente, J. van der Tol, H. Ambrosius, D. Robbins, M. J. Wale, N. Grote, and M. Schell, "A generic foundry model for inp-based photonic ics," in Optical Fiber Communication Conference, p. OM3E.3, Optical Society of America, 2012. - p.70.

[3] G. W. Steen, E. C. Fuchs, A. D. Wexler, and H. L. Offerhaus, "Identification and quantification of 16 inorganic ions in water by gaussian curve fitting of near-infrared difference absorbance spectra," Applied optics, vol. 54, no. 19, pp. 5937-5942, 2015. - p.70.

[4] W. E. Moerner and L. Kador, "Optical detection and spectroscopy of single molecules in a solid," Physical review letters, vol. 62, no. 21, p. 2535, 1989. - p.70.

[5] J. Angel and N. Woolf, "An imaging nulling interferometer to study extrasolar planets," The astrophysical journal, vol. 475, no. 1, p. 373, 1997. — p.71.

[6] K. Wallace, G. Hardy, and E. Serabyn, "Deep and stable interferometric nulling of broadband light with implications for observing planets around nearby stars," Nature, vol. 406, no. 6797, p. 700, 2000. - p.71.

[7] C. Hanot, B. Mennesson, S. Martin, K. Liewer, F. Loya, D. Mawet, P. Riaud, O. Absil, and E. Serabyn, "Improving interferometric null depth measurements using statistical distributions: theory and first results with the palomar fiber nuller," The Astrophysical Journal, vol. 729, no. 2, p. 110, 2011. — p.71.

[8] E. Serabyn, J. Wallace, H. Nguyen, E. Schmidtlin, and G. Hardy, "Nulling interferometry: working on the dark fringe," in Working on the Fringe: Optical and IR Interferometry from Ground and Space, vol. 194, p. 437, 1999. — p.71.

[9] P. Hlubina, M. Kadulová, and D. Ciprian, "Spectral interferometry-based chromatic dispersion measurement of fibre including the zero-dispersion wavelength," Journal of the European Optical Society-Rapid publications, vol. 7, 2012. — p.71.

[10] B. Yao, Y. Wu, Z. Wang, Y. Cheng, Y. Rao, Y. Gong, Y. Chen, and Y. Li, "Demonstration of complex refractive index of graphene waveguide by microfiber-based mach-zehnder interferometer," Optics Express, vol. 21, no. 24, pp. 29818-29826, 2013. - p.71.

[11] P. Labeye, J.-E. Broquin, J.-P. Berger, P. Kern, and P. Noël, "Silicon-based integrated optics for stellar interferometry imaging," in Silicon Photonics, vol. 6125, p. 61250O, International Society for Optics and Photonics, 2006. — p.71. 
[12] M. Stegmaier and W. H. Pernice, "Broadband directional coupling in aluminum nitride nanophotonic circuits," Optics Express, vol. 21, no. 6, pp. 7304-7315, 2013. - p.71.

[13] Z. Lu, H. Yun, Y. Wang, Z. Chen, F. Zhang, N. A. Jaeger, and L. Chrostowski, "Broadband silicon photonic directional coupler using asymmetric-waveguide based phase control," Optics express, vol. 23, no. 3, pp. 3795-3808, 2015. - p..

[14] J. Xing, K. Xiong, H. Xu, Z. Li, X. Xiao, J. Yu, and Y. Yu, "Silicon-on-insulatorbased adiabatic splitter with simultaneous tapering of velocity and coupling," Optics letters, vol. 38, no. 13, pp. 2221-2223, 2013. - p.71.

[15] H.-k. Hsiao, K. A. Winick, and J. D. Monnier, "Midinfrared broadband achromatic astronomical beam combiner for nulling interferometry," Applied optics, vol. 49, no. 35, pp. 6675-6688, 2010. - p.71.

[16] P. Labeye, Composants optiques intégrés pour l'Interférométrie astronomique. $\mathrm{PhD}$ thesis, Institut National Polytechnique de Grenoble-INPG, 2008. - p.71.

[17] A. S. Sudbo, "Film mode matching: a versatile numerical method for vector mode field calculations in dielectric waveguides," Pure and Applied Optics: Journal of the European Optical Society Part A, vol. 2, no. 3, p. 211, 1993. — p.71.

[18] S. Kim and A. Gopinath, "Vector analysis of optical dielectric waveguide bends using finite-difference method," Journal of lightwave technology, vol. 14, no. 9, pp. 2085-2092, 1996. - p.71.

[19] J. Saijonmaa and D. Yevick, "Beam-propagation analysis of loss in bent optical waveguides and fibers," JOSA, vol. 73, no. 12, pp. 1785-1791, 1983. — p.72.

[20] S. Rashleigh, "Origins and control of polarization effects in single-mode fibers," Journal of Lightwave Technology, vol. 1, no. 2, pp. 312-331, 1983. - p.72.

[21] G. Griffel, "Vernier effect in asymmetrical ring resonator arrays," IEEE Photonics Technology Letters, vol. 12, no. 12, pp. 1642-1644, 2000. — p.75.

[22] D. S. Fox, E. J. Opila, Q. N. Nguyen, D. L. Humphrey, and S. M. Lewton, "Paralinear oxidation of silicon nitride in a water-vapor/oxygen environment," Journal of the American Ceramic Society, vol. 86, no. 8, pp. 1256-1261, 2003. - p.87.

[23] J. Dalton and J. Drobek, "Structure and sodium migration in silicon nitride films," Journal of the Electrochemical Society, vol. 115, no. 8, pp. 865-868, 1968. - p.87. 

World within world [1]

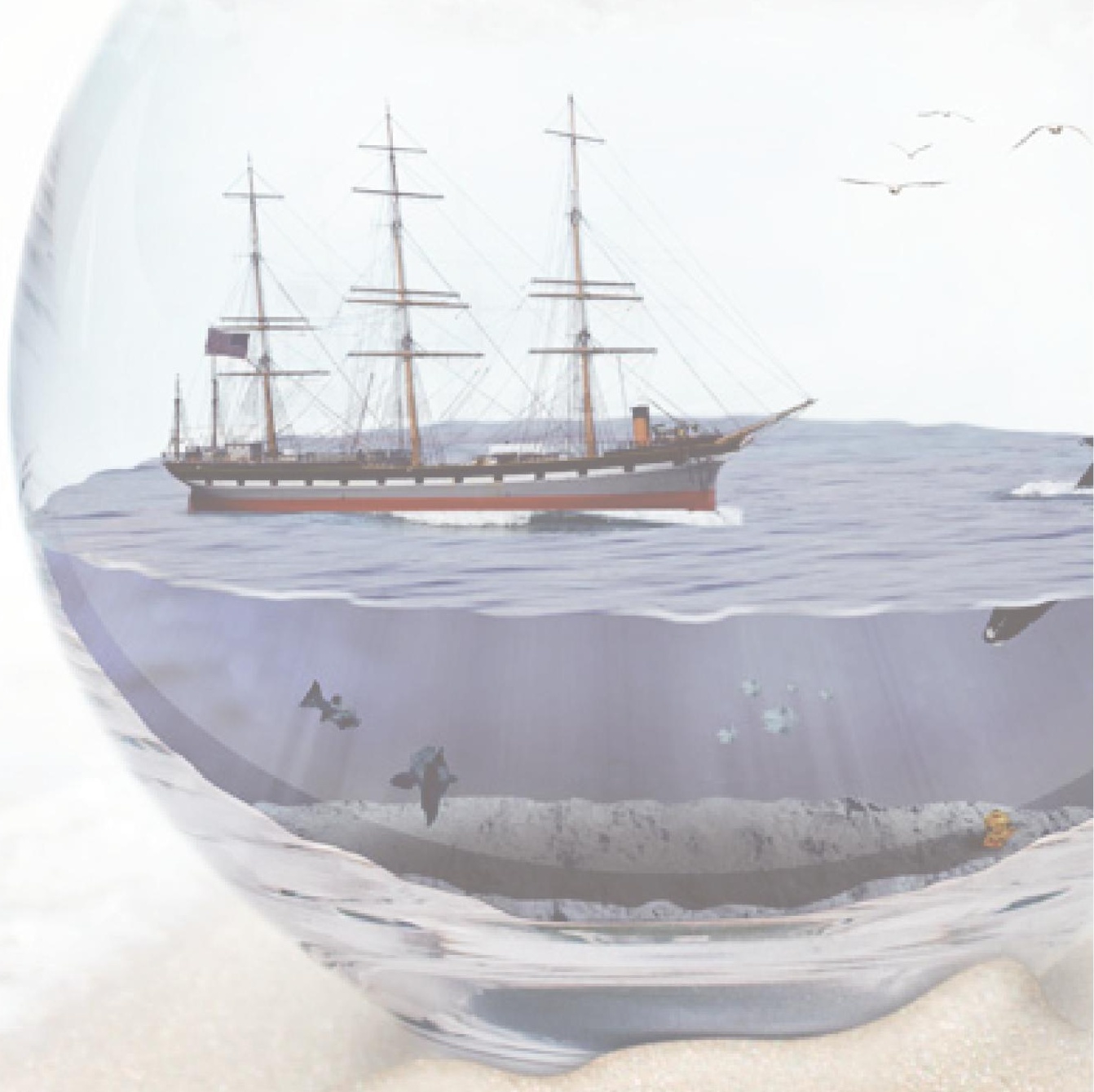




\section{CHAPTER}

\section{Optofluidic chip - designs and fabrication II}

George E.P. Box., Statistics for Experimenters, 1978

Subsections 5.1 - 5.7 have been accepted as G. W. Steen, E. F. Fuchs, A. D. Wexler, H. L. Offerhaus. "Design considerations to realize differential absorption based optofluidic sensors for determination of ionic content in water". IEEE Sensor. 2018. 


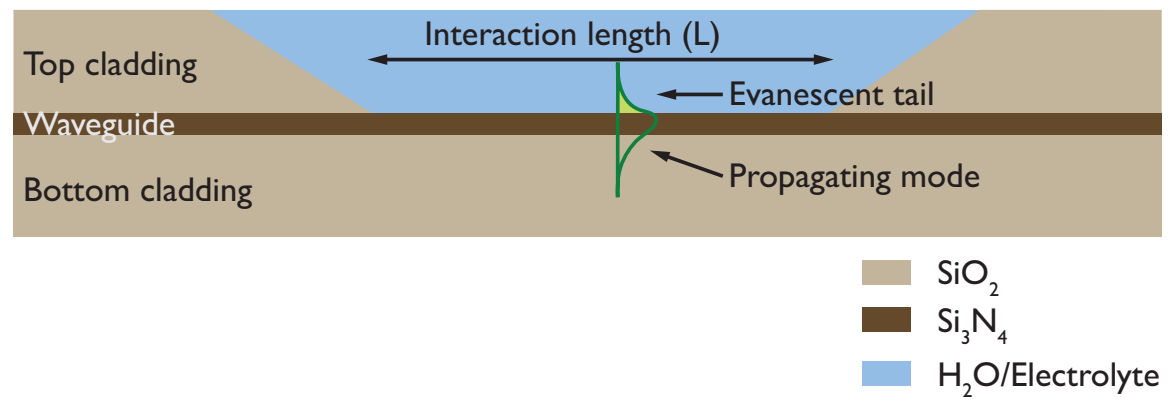

Figure 5.1: Schematic of the longitudinal section of the sensing window. Light is confined by the thin layer of $\mathrm{Si}_{3} \mathrm{~N}_{4}$ laying between a cladding of $\mathrm{SiO}_{2}$ on the wafer (silicon substrate). The top cladding of the TriPleX configuration is removed to enable sensing by interaction of the evanescent tail with the tail.

\subsection{Introduction}

Regulatory agencies such as the world health organization (WHO) and the environmental protection agency (EPA) have tightened the acceptable drinking standard as well as their reporting requirements. Inorganic ionic content is relevant for general safety of water consumption. Some ions (e.g., $\mathrm{Pb}^{2+}, \mathrm{Hg}^{2+}, \mathrm{Cd}^{2+}, \mathrm{Ti}^{3+}, \mathrm{As}^{3+}$ ) are toxic even in trace amounts. [2] The concentration of these ions should be monitored in real-time to avoid natural poisoning, as well as bioterrorism. [3] The use of an optofluidic sensor can help to meet these demands.

The standard technology to determine the ionic content in water is ion chromatography (IC). [4] It is time intensive and requires dedicated expertise. To overcome these obstacles IC on-chip devices are currently being developed. [5] Integrated optofluidic devices have a number of advantages over free space systems and are well suited for in-situ/in-line, real-time monitoring of water quality. [6]

In such a sensor both the fluidic and optical parts are miniaturized (microscale) and combined, ensuring on-/in line capabilities. The optical part of the sensor meets the real-time requirement. The microfluidic part is essential to probe the fluid. Part of the energy in the evanescent field of the optical mode is absorbed. Planar waveguides are positioned on the bottom of the microfluidic channels. Interaction with the fluid is enabled in the sensing window by removal of the protecting upper cladding layer (Fig. 5.1). The TriPleX platform allows a high evanescent field sensitivity in combination with low bend losses.

Low-loss light propagation through optofluidic devices is limited to the visible and near-infrared (VIS/NIR, $0.4 \mu \mathrm{m}$ to $2.6 \mu \mathrm{m}$ ) wavelength region. Direct detection of most mono-atomic ionic content in water is not feasible with a light source having these wavelengths. The interaction of light with ions of most dissolved inorganic salts is orders of magnitude lower than the interaction of its complex water environment (exceptions are transition metal ions). The aqueous absorption spectrum can be probed in the VIS/NIR wavelength regions and is known to be uniquely influenced by the presence of anions and cations. [7-10]

Previous work showed a potential utilization of a spectral window from $0.94 \mu \mathrm{m}$ 

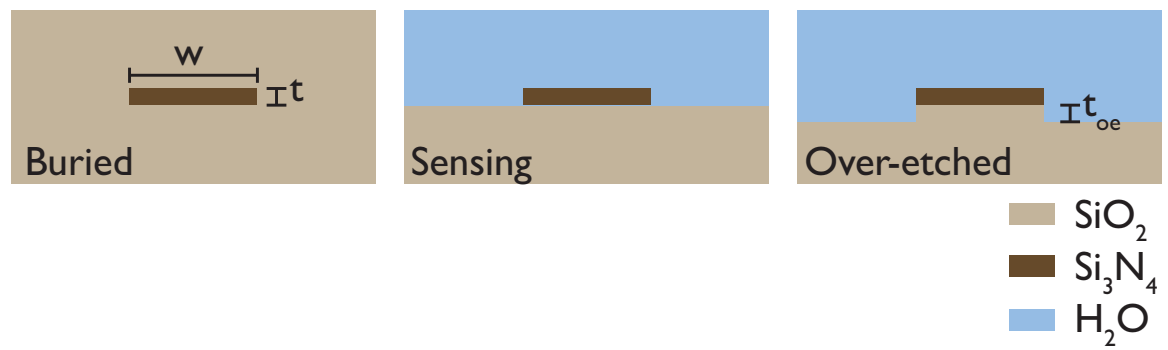

Figure 5.2: Schematic representations of different chip structures visualized with the geometric parameters: width $(w)$, thickness $(t)$, and over-etched depth $\left(t_{\mathrm{oe}}\right)$.

to $1.04 \mu \mathrm{m}$ for identification and quantification of electrolytes based on their specific perturbations of the water absorption spectrum. [11, 12] The same optical method can be applied to integrated optofluidic chips. Sensitivity to the fluid in the microfluidic channels is achieved by removal of the top cladding of the planar waveguides. The overlap of the zero-order mode to the fluid is roughly $\sim 20 \%$, i.e., the fraction of the energy that resides in the fluid compared to the total mode energy. The total interaction with the fluid (losses due to the evanescent tail probing the fluid) can be increased by fabricating a longer path length. The optical waveguides are customarily used in a spiral form to minimize the area used on the chip.

The sensors described in this work were designed in cooperation with LioniX to record differential on-chip NIR $(0.94 \mu \mathrm{m}$ to $1.04 \mu \mathrm{m})$ absorption. Waveguides with equal sensing areas are used to probe the absorption difference between two fluids contained in the microfluidic channels. The reference channel contains water and the sample channel contains water with ionic content.

This work presents cross-section optimizations of a silicon nitride $\left(\mathrm{Si}_{3} \mathrm{~N}_{4}\right)$ core embedded in silicon oxide $\left(\mathrm{SiO}_{2}\right)$ to enable single mode transverse electrical $\left(\mathrm{TE}_{0}\right)$ light propagation $(0.94 \mu \mathrm{m}$ to $1.04 \mu \mathrm{m})$ having high sensitivity, high tolerance for fabrication deviations and $<0.01 \mathrm{~dB} \mathrm{~cm}^{-1}$ bend losses for a radius of $500 \mu \mathrm{m}$. Furthermore, path lengths for $0.1 \mathrm{~dB} \mathrm{~cm}^{-1}, 0.5 \mathrm{~dB} \mathrm{~cm}^{-1}$ and $1.5 \mathrm{~dB} \mathrm{~cm}^{-1}$ propagation losses of waveguides were calculated to obtain the maximal differential signals; and were used in designs varying in sensitivity and complexity.

\subsection{Cross-section optimization}

The simplest geometry of the TriPleX technology was chosen to propagate the NIR light in the chips: a $\mathrm{Si}_{3} \mathrm{~N}_{4}$ core surrounded by a $\mathrm{SiO}_{2}$ cladding, henceforth called buried structure. The top cladding of the buried structure was removed by chemical etching to probe the fluid with the evanescent field in the sensing area of the chip, henceforth called sensing structure. The cross-sections of the buried and sensing structure are shown in figure 5.2. The figure also shows a simplified cross-section of an over-etched sensing structure.

The thickness of the $\mathrm{Si}_{3} \mathrm{~N}_{4}$ core can be well controlled $( \pm 2 \mathrm{~nm})$ with the etching steps in the fabrication process, while the width of the waveguides depends on the lithography 


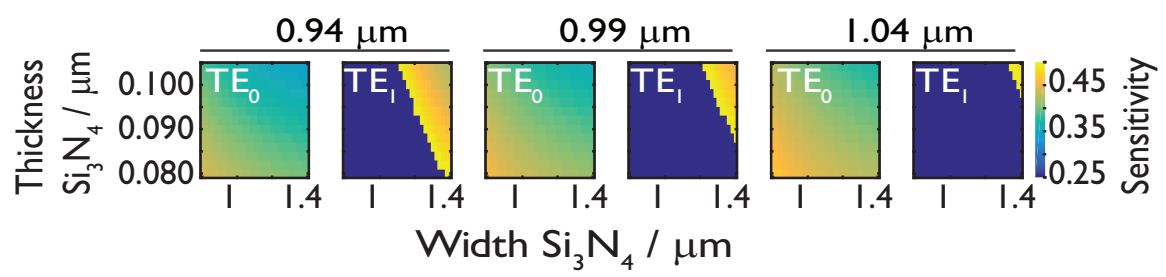

Figure 5.3: Color coded sensitivity of the $\mathrm{TE}_{0}$ and $\mathrm{TE}_{1}$ mode calculated for the buried geometry with the center wavelength and the outer wavelengths.

deviating $\sim 10 \%$. Over-etch depths are expected to be in the range of $50 \mathrm{~nm}$ to $150 \mathrm{~nm}$ based on a previous fabrication run. The thickness $(t)$ and width $(w)$ were optimized to obtain the following features for the broadband wavelength $(\lambda)$ range:

- A single mode operation,

- high sensitivity combined with a,

- reasonable tolerance for fabrication deviations,

- and $<0.01 \mathrm{~dB} \mathrm{~cm}^{-1}$ bend losses for a radius of $500 \mu \mathrm{m}$.

The Film Mode Matching (FMM) method was used to determine the mode profiles propagation through the different structures (sensing and buried). Sensitivity $(S)$ of differently propagating modes can be expressed by the ratio of the mode energy in the top cladding and the energy of the entire mode propagating through the structure. The sensitivity was calculated by determining the change in the effective refractive index due to a small induced change in refractive index of the fluid for the sensing structure. Likewise, the sensitivity of light propagating through the buried structure due to strain or heat on the top cladding was calculated by determination of the change in the effective refractive index due to the change in the refractive index $(n)$;

$$
S(\lambda, t, w)=\left(n_{e f f(n)}-n_{e f f(n+\Delta)}\right) \cdot \Delta^{-1},
$$

where the effective refractive index $\left(n_{\text {eff }}\right)$ was determined by the FMM method in "Optodesigner" (Phoenix) for the sensing structure with and without a small addition $\left(\Delta_{n}\right)$ to the refractive index of the fluid or top cladding in case of the buried structure.

The bend losses were determined by the Finite Difference (FD) method. The calculation of losses with the FD method requires more computational power compared to the FMM method. The mode is barely guided for small sizes of the $\mathrm{Si}_{3} \mathrm{~N}_{4}$ core, leading to low sensitivity and high bend losses. Therefore, the FMM method was first utilized to determine the parameters, i.e., the range of sizes of the $\mathrm{Si}_{3} \mathrm{~N}_{4}$ core that lead to single mode propagation with at least $17 \%$ sensitivity. The effective index method (EIM) was used to determine the effective refractive index for wavelengths with $0.01 \mu \mathrm{m}$ increments ranging from $0.9 \mu \mathrm{m}$ to $1.08 \mu \mathrm{m}$ of the $\mathrm{TE}_{0}$ mode for both the sensing and buried structures.

The methods are implemented using the 64-bit version of Optodesigner, licensed by Phoenix. The data were saved (with 15 significant digits) to a solid state drive. The software evaluated 300 different modes in the FMM method to ensure that the guided 


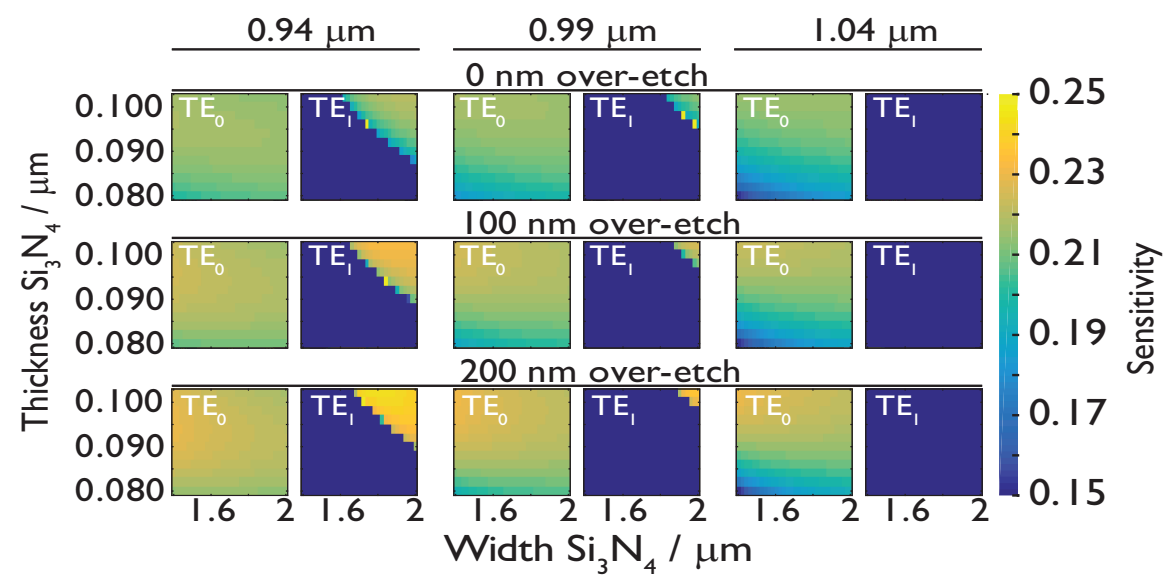

Figure 5.4: Color coded sensitivity of the $\mathrm{TE}_{0}$ and $\mathrm{TE}_{1}$ mode calculated for the sensing geometry with $0 \mathrm{~nm}, 100 \mathrm{~nm}$ and $200 \mathrm{~nm}$ over-etch depth.

modes were found. Convergence was verified for the parameters used (grid-size, number of modes, etc.).

The refractive indices at room temperature $\left(20^{\circ} \mathrm{C}\right)$ for water, thermally grown silicon dioxide, single-crystal silicon and low-pressure chemical vapor deposition (LPCVD) stoichiometric silicon nitride were provided by LioniX. The sensitivity of the propagating mode of the buried structure was determined for the different sizes of the $\mathrm{Si}_{3} \mathrm{~N}_{4}$ layer and is presented by color coding in figure 5.3. The waveguide dimensions can be defined for single mode operation from this figure.

The sensitivity was determined for three wavelengths $0.94 \mu \mathrm{m}, 0.99 \mu \mathrm{m}$ and $1.04 \mu \mathrm{m}$ to define the spectral response within the $100 \mathrm{~nm}$ bandwidth of the sensor. The sharp cut-off in sensitivity seen in the $2 \mathrm{D}$ color-coded figure of the $\mathrm{TE}_{1}$ mode gives a visual impression of the size of the $\mathrm{Si}_{3} \mathrm{~N}_{4}$ for which the mode becomes guided.

The sensitivity of the $\mathrm{TE}_{0}$ and $\mathrm{TE}_{1}$ modes calculated for the sensing structure is presented in figure 5.4 for wavelengths $0.94 \mu \mathrm{m}, 0.99 \mu \mathrm{m}$ and $1.04 \mu \mathrm{m}$, respectively, in three distinct situations: perfect removal of the top cladding, $100 \mathrm{~nm}$ over-etch and $200 \mathrm{~nm}$ over-etch of the top cladding. Light of shorter wavelengths is better confined than the light of longer wavelengths which can be seen in the figures. The increase in sensitivity due to a deeper over-etch is visible in the first order mode for a wavelength of $0.94 \mu \mathrm{m}$.

Two suitable combinations of height and width that display high sensitivity and high foundry tolerance in the width of the $\mathrm{Si}_{3} \mathrm{~N}_{4}$ core were found for single mode propagation based on the data presented in figures 5.4 and 5.3. The first combination has a width of $(1.95 \pm 0.15) \mu \mathrm{m}$ and a thickness of $(83 \pm 2) \mathrm{nm}$ and the second combination has a width of $(1.7 \pm 0.1) \mu \mathrm{m}$ and a thickness of $(92 \pm 2) \mathrm{nm}$, respectively. These combinations balance fabrication tolerance in width against bend losses. 


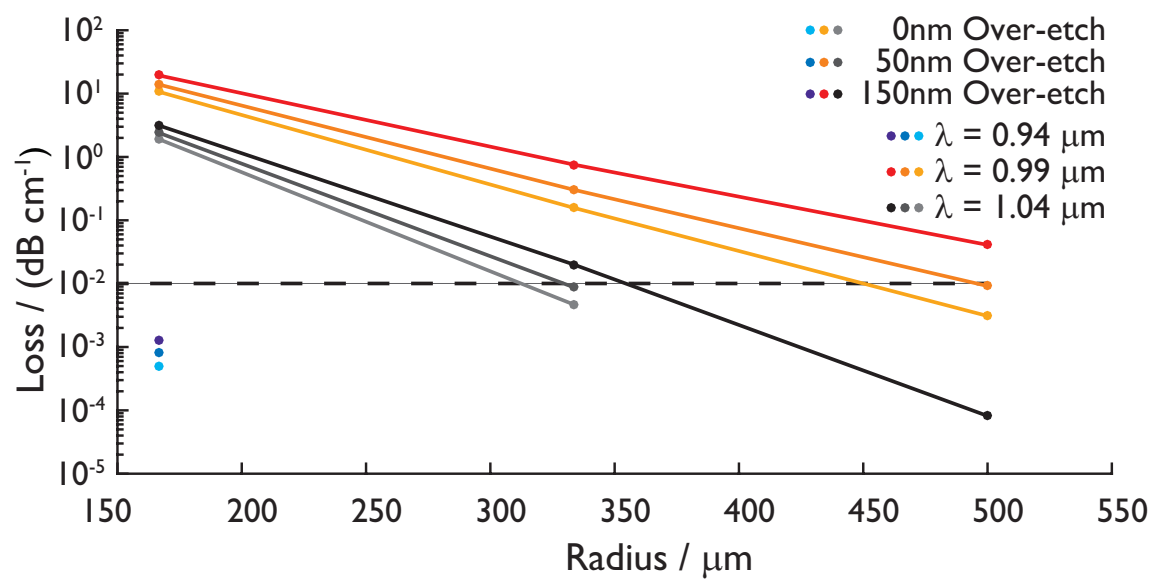

Figure 5.5: Bend losses determined for different radii, wavelengths, and over-etch depths. The dashed line indicates the desired $<0.01 \mathrm{~dB} \mathrm{~cm}^{-1}$ bend losses for a radium of $500 \mu \mathrm{m}$. Due to the FD limitation of the simulations only the bend losses for a radius of $166 \mu \mathrm{m}$ are displayed for wavelength $0.94 \mu \mathrm{m}$.

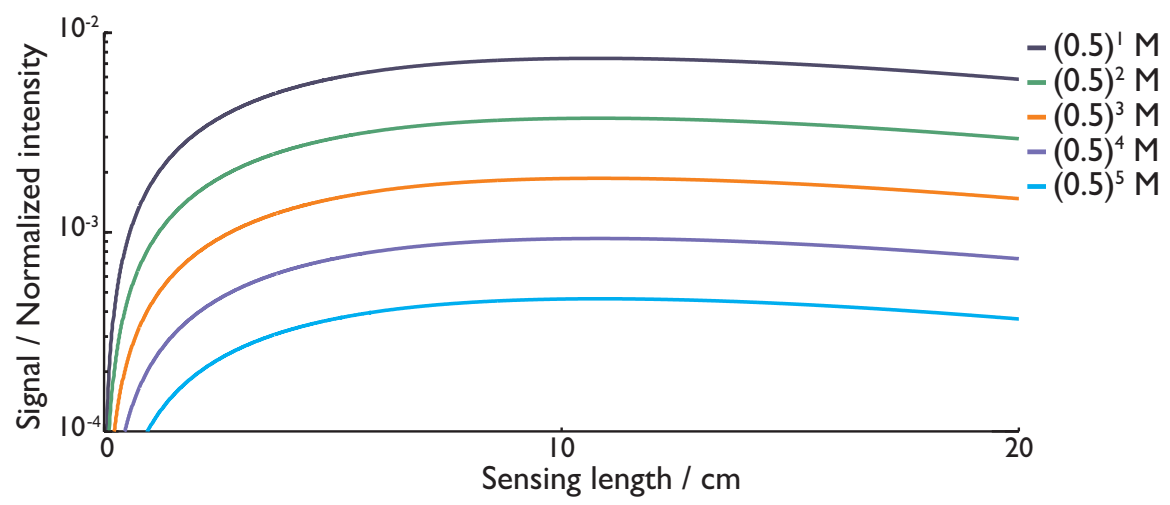

Figure 5.6: Expected normalized intensities of different concentrations of ionic content against sensing lengths when $20 \%$ of the propagating light is exposed to the fluid.

\subsubsection{Bend losses}

The losses due to bend radii of $150 \mu \mathrm{m}, 333 \mu \mathrm{m}$ and $500 \mu \mathrm{m}$ were determined for the $\mathrm{TE}_{0}$ mode with wavelength $0.94 \mu \mathrm{m}, 0.99 \mu \mathrm{m}$ and $1.04 \mu \mathrm{m}$ for the sensing structure with $0 \mathrm{~nm}$, $50 \mathrm{~nm}$ and $150 \mathrm{~nm}$ over-etch and the sizes of the two regions. The spectral absorption of water was included in the simulation to determine the band losses for a sensing structure with a $\mathrm{Si}_{3} \mathrm{~N}_{4}$ core thickness of $(92 \pm 2) \mathrm{nm}$ and width of $(1.6 \pm 0.2) \mu \mathrm{m}$. The spectral loss due to water of a straight sensing waveguide were subtracted to determine the losses due to bend radii.

Figure 5.5 shows the bend losses for a $\mathrm{TE}_{0}$ mode propagating through a sensing structure with a $\mathrm{Si}_{3} \mathrm{~N}_{4}$ core of $1.6 \mu \mathrm{m}$ by $92 \mathrm{~nm}$. The $<0.01 \mathrm{~dB} \mathrm{~cm}^{-1}$ bend losses for a radius of $500 \mu \mathrm{m}$ can be achieved for entire spectral range and over-etch depth of $50 \mathrm{~nm}$. 
The sizes of the $\mathrm{Si}_{3} \mathrm{~N}_{4}$ core in the second region led to ten times higher bend losses. Therefore, the parameters of the first region were used for the chip fabrication although the parameters of the second region would give an additional $0.1 \mu \mathrm{m}$ foundry tolerance in the width of the $\mathrm{Si}_{3} \mathrm{~N}_{4}$ core.

The fraction of the mode overlaps with the fluid increases with over-etch; and bending of a waveguide leads to a spatial shift of the propagating mode (e.g., a bend to the left would cause a shift of the mode to the right, deeper into the fluid). The increase of mode sensitivity to the water at a certain radius is therefore expected to increase with the over-etch depth; and with that, the losses are expected to increase. Figure 5.5 confirms that deeper over-etch leads to a higher bend loss.

The bend losses for the shorter wavelengths are smaller than those of the longer wavelengths in the absence of absorption due to better confinement of the light. Additional loss originates from the second overtone of the $\mathrm{OH}$ vibration, whose absorption maximum lies at $0.97 \mu \mathrm{m}$ and falls off steeply to either side.

\subsection{Sensing length}

The transmission difference due to the presence of ions in water is small; only $\sim 0.012 \cdot c$ of the total intensity for a $1 \mathrm{~cm}$ optical path, where $c$ is the concentration of ions in mol L ${ }^{-1}$. The intensities of the differential transmission $\left(I_{\mathrm{s}}\right)$ for different path lengths can be calculated using the Beer-Lamberts law: [13]

$$
I_{s}=10^{-A_{\text {water }}}-10^{-A_{\text {water }}(1 \pm(\delta \cdot c))},
$$

where $A_{\text {water }}$ is the spectral absorption of demineralized water, $c$ is the concentration in $\mathrm{mol} \mathrm{L}^{-1}$ and $\delta$ is the unique spectral effect (fingerprint) which the ions exert on the absorption spectrum of water. For $970 \mathrm{~nm}$ this $\delta$ is in the order of 0.04 for most electrolytes, and the optical density of water for this wavelength is approximate 0.2 . This simplifies (2) to define the differential transmission by the influence of both the geometrical path length (L) and the concentration of ions:

$$
I_{s}=10^{-0.2 L}-10^{-0.2 L(1 \pm(0.04 \cdot c))}
$$

The detection limit of a sensor based on differential absorbance is proportional to its dynamic range and can be maximized by optimizing the optical path length. Figure 5.6 presents normalized expected differential signal intensities plotted against the sensing length for different concentrations, $0.5 \mathrm{M}, 0.25 \mathrm{M}, 0.13 \mathrm{M}, 0.06 \mathrm{M}$ and $0.03 \mathrm{M}$, respectively. The concentration of the electrolytes exerts an effect on the expected normalized signal as one would expect; however, the optimum sensing length for these concentrations seems to be not significant affected. The optimum length $\left(L_{\mathrm{m}}\right)$ can be obtained by solving the derivative of $T_{\mathrm{s}}$ with respect to $L$ and setting the derivative to zero,

$$
\begin{gathered}
\frac{d T_{s}}{d L}=\frac{c+25}{125} \log _{e}(10) \cdot 0.1^{(c+25) / 125}-0.2 \log _{e}(10) \cdot 0.1^{0.2 L}, \\
L_{m}=\frac{125}{c} \log _{10}\left(\frac{c}{25}+1\right) .
\end{gathered}
$$




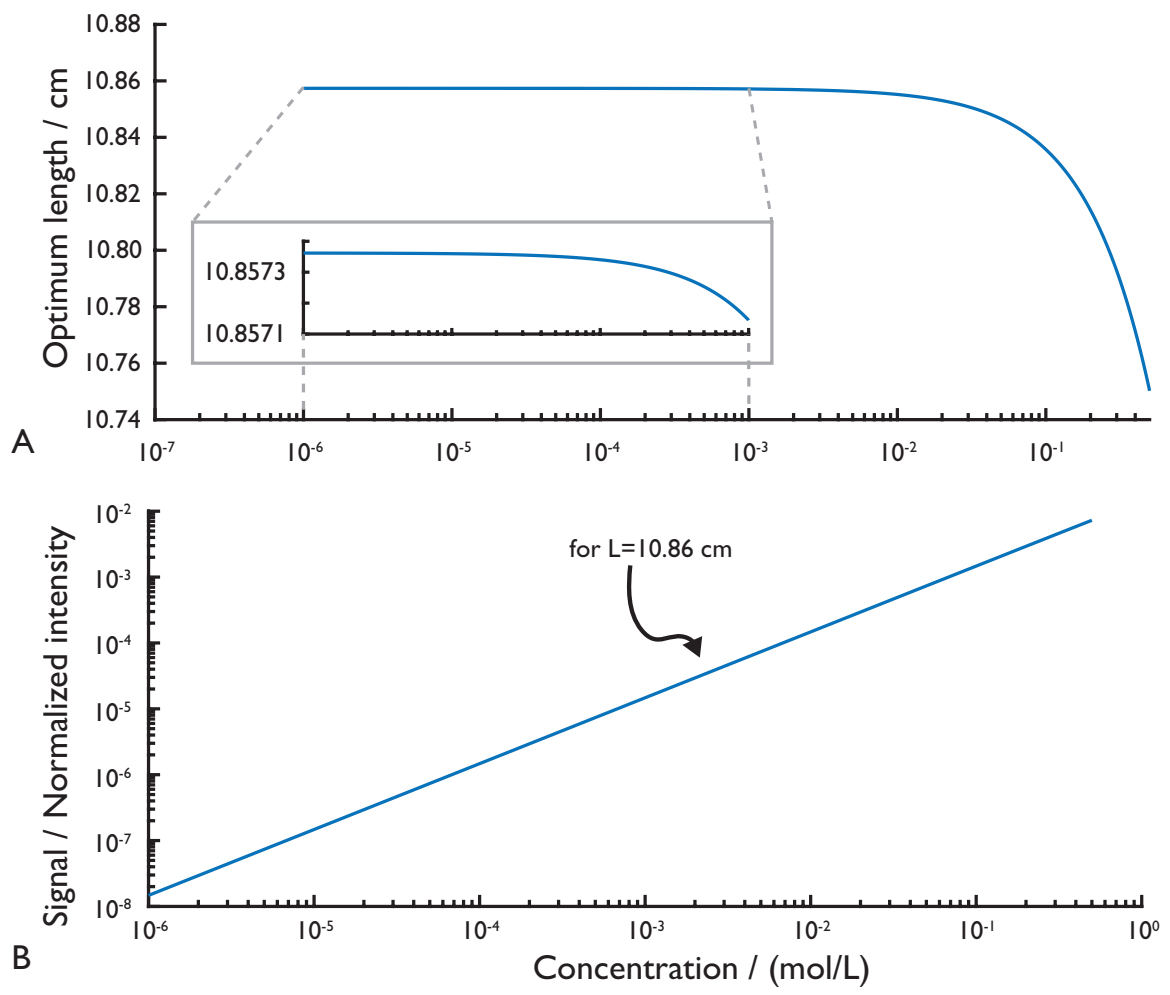

Figure 5.7: (A) Optimum integrated geometric sensing path length against electrolyte concentration. (B) Expected normalized signal against concentration for an optimized length of 10.86 $\mathrm{cm}$.

The optimum geometrical length (5.5) for differential absorption measurements between two similar fluids is approximately $2.17 \mathrm{~cm}$ for lossless propagation through air, mirrors and cuvettes. This path length leads to a six-fold increase of the differential transmission compared to a path length of $1 \mathrm{~cm}$. To translate the optimum geometric path length through a cuvette to the optimum integrated geometric sensing path length on the optical chips without waveguide losses (due to roughness of the $\mathrm{Si}_{3} \mathrm{~N}_{4}$ layer, absorption losses due to $\mathrm{SiO}_{2}$ and $\mathrm{Si}_{3} \mathrm{~N}_{4}$, etc.), the result of (5.5) is multiplied by five to account for the $20 \%$ fraction of the propagating mode in the fluid in the sensing windows.

The optimum sensing length is independent of the electrolyte concentration due to the small effect on the absolute absorption as can be seen in figure 5.7. A model that includes the waveguide losses is shown in figure 5.8. Incorporation of these losses significantly reduces the optimum sensing length. The silicon-based platform TriPleX allows low-loss propagation and small bend radii. For example, a loss of $0.095 \mathrm{~dB} \mathrm{~cm}^{-1}$ was recorded at a waveguide radius of $70 \mu \mathrm{m}$ for light with a wavelength of $1.55 \mu \mathrm{m}$. [14] However, the etching required for sensing leads to the higher surface roughness of the waveguides and therefore also higher propagation losses are expected. Three sensing lengths were chosen to account for different propagation losses $2.5 \mathrm{~cm}, 5 \mathrm{~cm}$ and $7.5 \mathrm{~cm}$. 


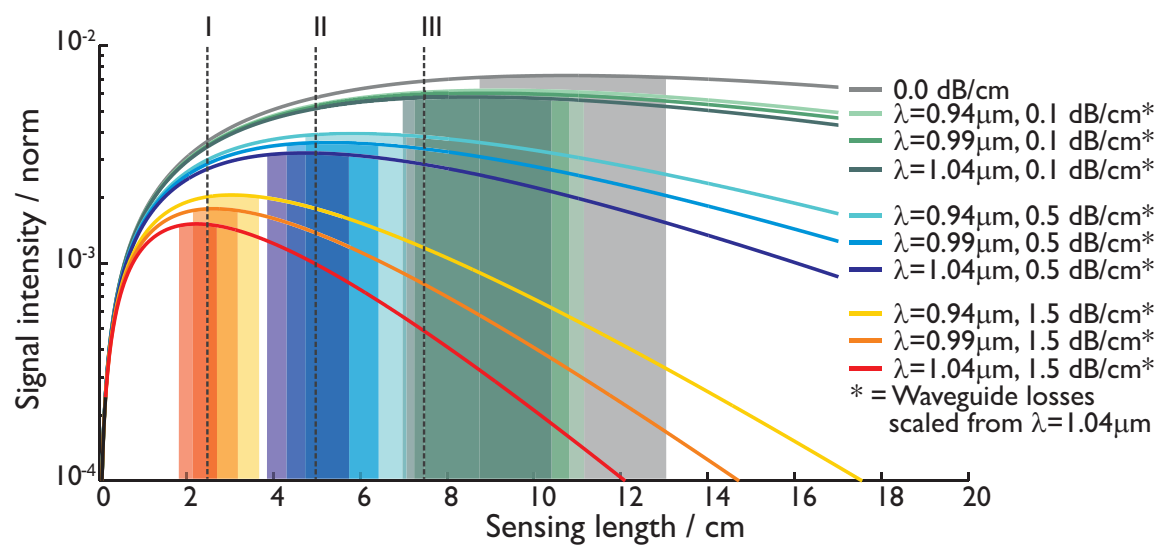

Figure 5.8: The expected normalized intensity of a $0.5 \mathrm{M}$ electrolyte is plotted against sensing length. The gray line presents a waveguide without losses; the green, blue and orange lines present waveguides with losses of $0.1 \mathrm{~dB} \mathrm{~cm}^{-1}, 0.5 \mathrm{~dB} \mathrm{~cm}^{-1}$ and $1.5 \mathrm{~dB} \mathrm{~cm}^{-1}$ at $\lambda=1 \mu \mathrm{m}$, respectively. The wavelength dependent losses are calculated with the scaling factor $\left(\lambda_{0} / \lambda_{1}\right)^{4}$. Sensing lengths chosen for chips designs are shown as dotted lines I, II and III plotted at $2.5 \mathrm{~cm}$, $5.0 \mathrm{~cm}$ and $7.5 \mathrm{~cm}$, respectively. The shaded areas represent $95 \%$ of the signal intensity for a given set of parameters. Short sensing lengths are appropriate for high waveguide losses.

\subsection{Interferometric detection}

Interferometric mixing was explored in different designs to improve the detection limit of the signal. A Mach Zehnder (MZ) interferometer with a $\pi$ phase difference between its two arms would produce complete destructive interference on one output (the 'dark' port). Half of the intensity difference in transmission of both arms, due to a difference in absorption, is directly measurable at the dark port. Naturally, ions in water influence both the real and imaginary parts of the complex refractive index of water.

Therefore, not only a perfect 50/50 splitting ratio of the coupling devices is required with a broadband $\pi$ shifter, but also the difference in the real part of water with that of an electrolyte needs to be compensated. Heaters are positioned above the buried waveguide and used to affect the effective refractive index of the waveguide (in the reference path) to compensate the difference in dispersion due to the presence of ions in the sample path. The increase of optical path length due to the presences of ions can be compensated by increasing the temperature of the $\mathrm{SiO}_{2}$ in the other interferometric arm, thereby increasing its optical path length. The compensation can also be achieved by a temperature offset between the reference and sample fluids. But, the change in spectral absorbance of the water due to this temperature difference needs to be accounted for. $[15,16]$

Differences in path length can be realized in other configurations of the MZ and so different number of spectral fringes can be recorded at the output ports of the MZ. The spectral fringes are influenced by the spectral phase relation between the two arms of the MZ. Fringe resolution is dependent in part on the modulation depth (i.e., the contrast on the chip). The number of fringes is linearly dependent on the change of the refractive index and on the optical path length difference. A change in the fringe spacing can be used to quantify the ionic concentration. The shape of the spectral envelop allows the 


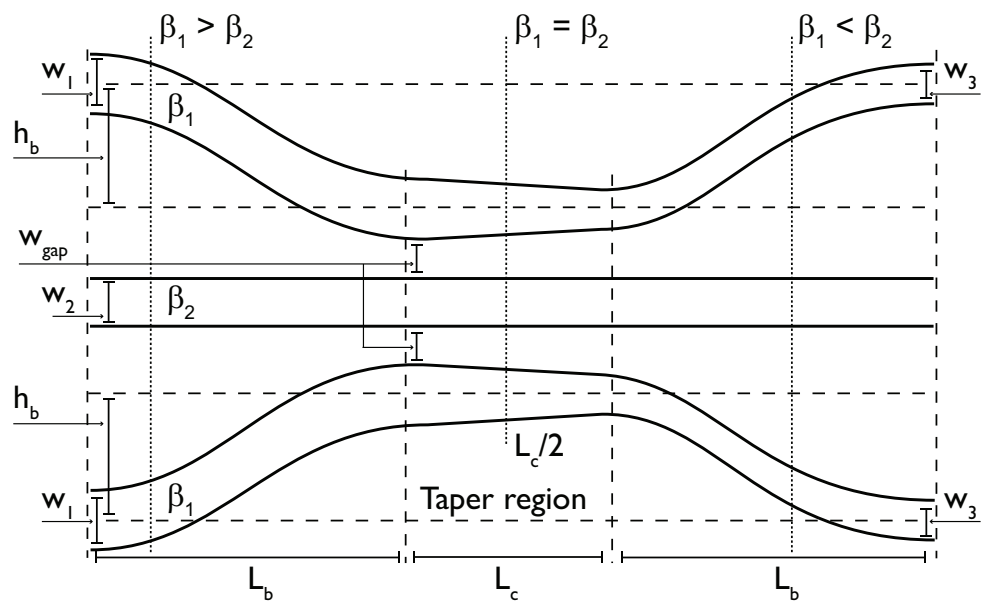

Figure 5.9: The $\Xi$ coupler with relevant parameters: the width of the gap ( $\left.w_{\text {gap }}\right)$ of $1 \mu \mathrm{m}$, the width of the outer waveguides $\left(w_{1}\right)$ equal to the width of the middle waveguide $\left(w_{2}\right)$ plus a delta width ( $\Delta W$ ) before tapered to $w_{2}$ minus the $\Delta W$, the height of the bend $\left(h_{\mathrm{b}}\right)$ equal $5 \mu \mathrm{m}$, the length of the bend $\left(L_{\mathrm{b}}\right)$ and the length of the coupling $\left(L_{\mathrm{c}}\right)$.

identification of the ionic constituents by differential absorption. [17, 18]

The deepest point of the destructive interference depends on the number of fringes in the spectral region in combination with the spectral resolution of the recording instruments. It is essential to accurately measure the depth, as it signifies the absorption imbalance. The coherence length of the laser and the spectral resolution of a spectrometer limit the fringe depth. Ionic content will reduce fringe depth. To accurately record the modulation two approaches were taken. First, we offset the optical path by three separate designs to compensate for low $(60 \mathrm{mM})$, medium $(0.5 \mathrm{M})$ and high $(1 \mathrm{M})$ ionic concentrations. Differences in path length were chosen to enable 10, 26 and 52 fringes in the spectral region. Second, heaters in the buried structure were used to shift the optical path length at least one full fringe. The first approach requires a spectrometer with a high spectral resolution but simplifies chip design.

The coherence length of the laser should be considered when determining the differential absorbance in both approaches. The following equations were used to determine suitable path difference of the buried waveguides between the two arms:

$$
\begin{gathered}
\Delta_{M Z}(\lambda)=n_{r b} L_{r b}-n_{s b} L_{s b}+n_{r f} L_{r f}-n_{s f} L_{s f}, \\
\Delta_{M Z}^{g}(\lambda)=N_{r b} L_{r b}-N_{s b} L_{s b}+N_{r f} L_{r f}-N_{s f} L_{s f},
\end{gathered}
$$

where $L$ is the geometric path length, $n$ the effective refractive index and $N$ the group effective refractive index. The sub-indices $r b, s b, r f$ and $s f$ signify reference buried, sample buried, reference fluid and sample fluid, respectively. The path lengths of both sensing waveguides were set to be of the same length. A phase shift was induced by 


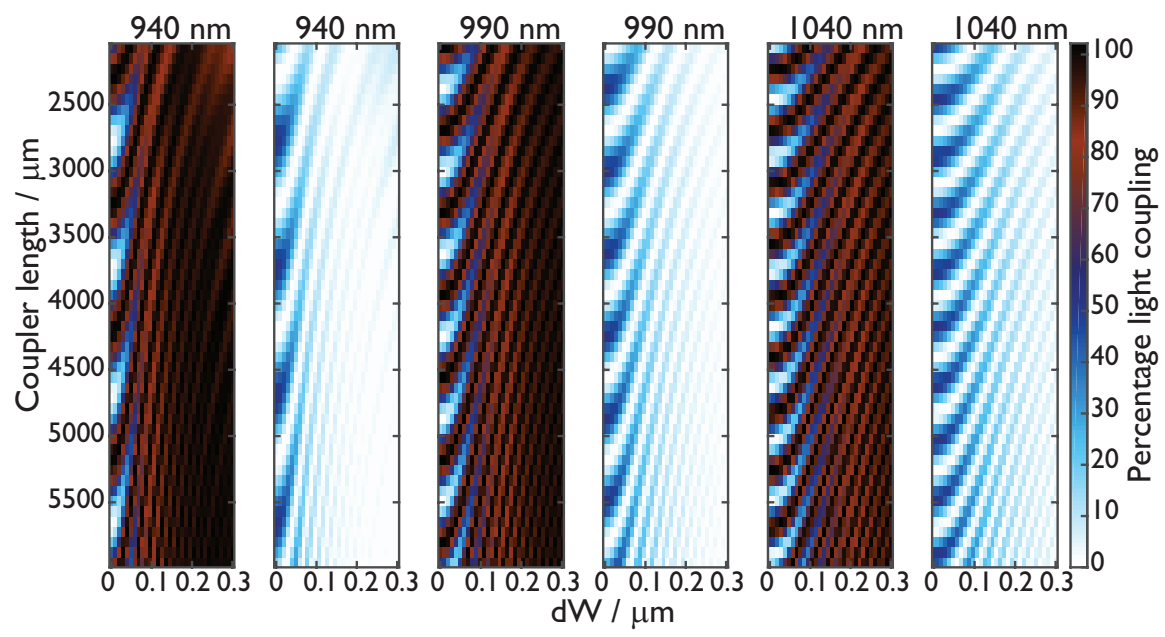

Figure 5.10: Effect of the change in width and coupler length for three different wavelengths on light propagation through a $\Xi$ coupler. For each wave length, the left graph and right graph show the percentage of light coupling to the outer waveguides and middle waveguide of the $\Xi$ coupler, respectively.

designing a difference in the length of the buried waveguides. The spectral signal $\left(\mathrm{S}_{\mathrm{MZ}}\right)$ can be calculated (see Appendix B.1) using

$$
S_{M Z}(\lambda)=y(1+V) \cos \frac{2 \pi \Delta_{M Z}(\lambda)}{\lambda} e^{-\left(\pi \Delta_{M Z}^{g} \Delta_{R} / \sqrt{2} \lambda^{2}\right)^{2}}
$$

where the width of the spectrometer response function $\Delta R$ is set to 0.23 and $0.04 \mathrm{~nm}$, $V$ is the visibility term and $y$ is normalized spectral source. A path length difference of $0.3 \mu \mathrm{m}, 59.5 \mu \mathrm{m}, 150 \mu \mathrm{m}$ and $300 \mu \mathrm{m}$ leads to $0,10,27$ and 53 spectral fringes, respectively.

\section{$5.5 \Xi$ coupler}

A $\Xi$ coupler is a bidirectional coupler with an extra waveguide in the middle. It avoids the inherent loss of y-splitters for recombination of light. The dark output port of a $y$-splitter is the unguided propagation mode in the cladding, inaccessible to record. The symmetric design of the $\Xi$ coupler yields three in- and outputs ports with equal power at the outer output ports when the light is coupled to the middle input port. However, the light intensity at the outer waveguides depends on the coupling length and spectral input. The phase relation can be kept constant by adiabatic coupling as shown in figure 5.9. The adiabatic coupling leads to a flattened intensity response with respect to the length of coupling and wavelength. Both y-splitters and $\Xi$ coupler were incorporated in the chip design to achieve achromatic bisection of light. 
Table 5.1: Parameters $\Xi$ coupler

\begin{tabular}{llll}
\hline & $\mathrm{dW}(\mu \mathrm{m})$ & $\mathrm{L}_{\text {coupler }}(\mu \mathrm{m})$ & $\mathrm{L}_{\text {bend }}(\mu \mathrm{m})$ \\
\hline$\# 1$ & 0.1 & 2500 & 875 \\
$\# 2$ & 0.2 & 2500 & 875 \\
$\# 3$ & 0.3 & 2500 & 875 \\
$\# 4$ & 0.1 & 3250 & 875 \\
$\# 5$ & 0.2 & 3250 & 875 \\
$\# 6$ & 0.3 & 3250 & 875 \\
$\# 7$ & 0.1 & 3250 & 1500 \\
$\# 8$ & 0.2 & 3250 & 1500 \\
$\# 9$ & 0.3 & 3250 & 1500
\end{tabular}

To evaluate the light propagation through the $\Xi$ coupler the beam propagation method (BPM) was used to obtain the percentage of light propagating in the different waveguides due to the relevant parameters ( $d W, L$ and $\lambda$ ) of the $\Xi$ coupler. Figure 5.10 shows the light propagation dependence through a $\Xi$ coupler with different coupling length, gap size, bending length and change in width. The $\Xi$ coupler was designed for the wavelength region of interest, based on designs presented by Hsien-kai Hsiao, et al. [19] Table 5.1 shows the parameters used for the different $\Xi$ couplers in the chip design.

\subsection{Designs}

Figure 5.11 shows an overview of the schematic designs. Design 11,12 , and $13\left(\mathrm{D}_{11-13}\right)$ contain electronic subtraction schemes, whereas the other designs are interferometric to perform the subtraction optically. Table I presents the parameters of the $\Xi$ couplers in design $\mathrm{D}_{21-23}$ and $\mathrm{D}_{41-43} . \mathrm{D}_{21-23}$ replaces the y-splitter with a $\Xi$ coupler to enable a Michelson interferometer on-chip reducing the required sensing path by a factor of 2 . The difference in path length was 59.5 and $150 \mu \mathrm{m}$ for the two Michelson interferometers placed on each chip. Designs $D_{31 a-34 a}$ feature a Mach Zehnder layout with a path difference of $0.3 \mu \mathrm{m}, 59.5 \mu \mathrm{m}, 150 \mu \mathrm{m}$ and $300 \mu \mathrm{m}$, respectively. Design $\mathrm{D}_{31 \mathrm{~b}-34 \mathrm{~b}}$ utilizes a homodyne interferometric layout and design $\mathrm{D}_{41-43}$ uses an identical layout of the Mach Zehnder, but has a $\Xi$ coupler to recombine both light paths. $\mathrm{D}_{51}$ and $\mathrm{D}_{52}$ were designed to enable online calibration of the sensor. The mask designs used to realize these designs are presented in Appendix D.

\subsection{Fabrication}

The chips were fabricated by Lionix according to the design's specifications of this work and allowed single mode propagation of the designed spectral bandwidth. To ease the effort of aligning the fiber array to the chip gold lines were deposited on top of the chip, located between the waveguides, to visually mark the position of the embedded waveguides. The etching area was chosen to be as small as possible to avoid 

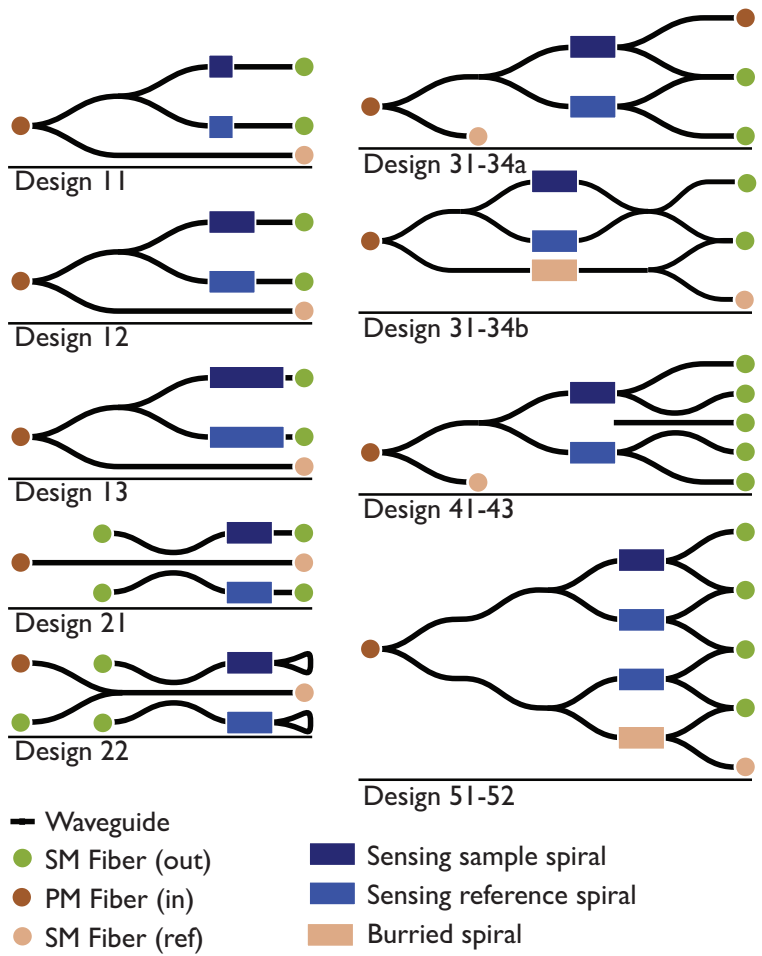

Design 51-52

Sensing sample spiral

Sensing reference spiral

Burried spiral

Figure 5.11: Five designs are schematically presented. The heaters are not drawn for simplicity. Design $\mathrm{D}_{11-13}$ are placed upon one die (chip). Similar, two Michelson inteferometers $\left(\mathrm{D}_{21-23}\right)$ are placed on one die. The chips were designed for one standardized fiber array (3 PM fibers and 9 SM fibers) to minimize the cost. Every design exhibits fluidic windows for sensing of the sample and the reference fluid water (dark- and light blue, respectively). Design 1 is based on non-interferometric method of detection. Design 2 is a Michelson interferometer (reducing path length by two). Design 3 is a Mach Zehnder interferometer with a y-splitter for recombination of the bisected light, while in design 4 a $\Xi$ coupler is used. Design 5 uses a double Mach Zehnder interferomter to increase signal stability.

an inconsistent top layer close to the interface of buried and sensing structure, i.e., the top cladding above the spirals was removed instead of the entire window.

\subsection{Optical throughput chips - second fabrication}

\subsubsection{Materials}

The setup described in subsection 4.5.1 was used with some small modifications. First, an integrated isolator (standard IO-J-980) was installed by Superlum in the broadband SLD (BLMS-mini-521-HP, Superlum ${ }^{\mathrm{TM}}$, Carrigtwohill, Co. Cork, Ireland) to reduce optical feedback to the source. Second, they swapped the TE/TM axes using a fiber splicer. Thus, the cross-PM fiber was removed from the setup to butt-coupled the light from the source directly to the input fiber of the fiber-array (FA). Finally, an autosampler 


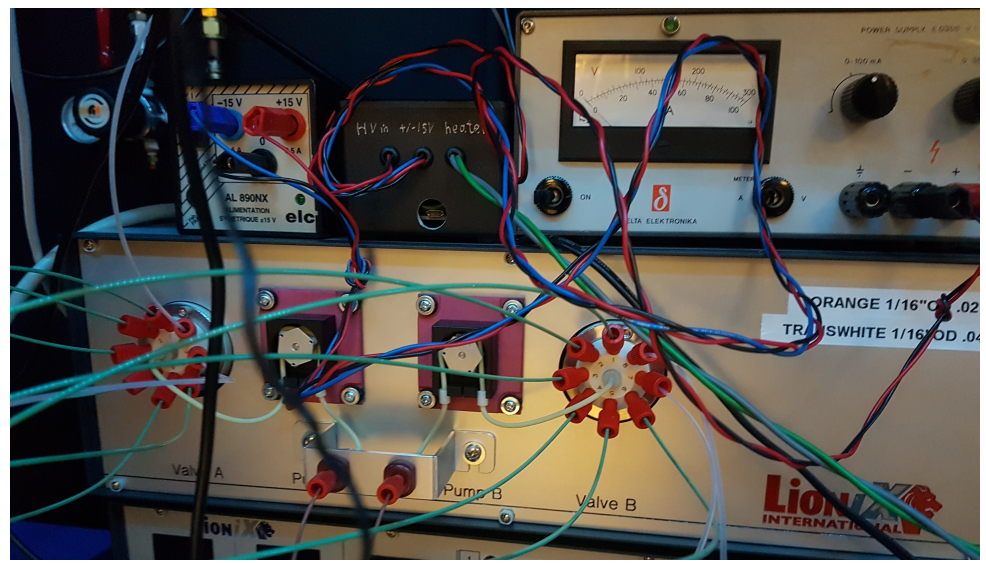

Figure 5.12: Fotograph of the autosampler built by LioniX to flush the fluidic windows with solutions. On top of the autosampler the heater control units are located.

(Fig. 5.12) was built by LioniX to replace the syringe pumps. The device consisted of two peralistic pumps (p625/900.133) with 8 rotary valves (eight outlets and one inlet). The pumps could be separately controlled with an Arduino using LabVIEW software. Standard tubes (PEEK and 20P Pharmed, VWR, Amsterdam, NL) $50 \mathrm{~cm}$ were used to transport solutions stored in $50 \mathrm{~mL}$ bottles to the fluidic windows on the chips.

The $50 \mathrm{~mL}$ solutions of $0.0613 \mathrm{M}, 0.125 \mathrm{M}, 0.25 \mathrm{M}, 0.5 \mathrm{M}$ and $1 \mathrm{M} \mathrm{Na}_{2} \mathrm{SO}_{4}$ were prepared using demineralized water, precision $( \pm 0.1 \mathrm{mg})$ balance (PIONEER ${ }^{\mathrm{TM}}$, Ohaus, Florham Park, NJ, USA) and reagent or higher grade salts (Sigma Aldrich, St Louis, MO, USA). The solutions were flushed through $0.45 \mu \mathrm{m}$ filters (Millipore Millex-LCR, VWR, Amsterdam, NL) to prevent clogging.

\subsubsection{Procedure}

Two spectrometers (AvaSpec-ULS3648 StarLine High-resolution, Avantes, Apeldoorn, NL) with optical resolutions of $\sim 0.6 \mathrm{~nm}$ were used to record the optical transmission. One monitored the spectral power fluctuation and the other the transmission of the MZI (design 31a-34a), the second MZI on-chip (design 31b-34b), and the homodyne interferometer (design 31b-34b). Water was flushed multiple times through to chips, after which 200 spectra of the different output were recorded.

The sensitivity of three sensors were determined by recording the influence of dissolved salt on the transmission spectrum of water. First, pure water was flushed multiple times through the chips, before 200 baselines were recorded. Next, the sample window was flushed by the solution to be measured ( 5 times $1 \mathrm{~mL}$ ). Then 200 additional spectra were collected. This step was repeated for each sample until the highest concentration (1M) was reached. Finally, the sample window was flushed with distilled water and 200 spectra were recorded. The solutions were automatically and sequentially flushed through the top window (using the autosampler) and repeated for the bottom window of the optofluidic chips.

The recorded spectral counts transmitted through the chips with interferometric design 


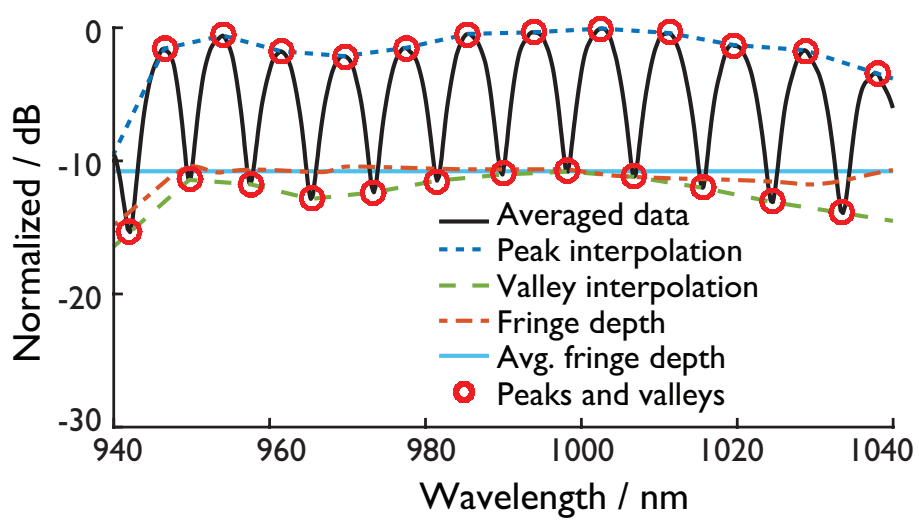

Figure 5.13: Visual example of data processing algorithm. The peak and valleys of the fringes are located in the averaged data (design 32, wafer W4259). From the values the average fringe depth over a $100 \mathrm{~nm}$ is determined.

were normalized and converted to the $\mathrm{dB}$ scale with a MATLAB program (R2017b, the MathWorks, Natick, MA, USA). Figure 5.13 show the next steps in the data analysis. First, the peaks and valleys of the fringes are determined. Then the peaks and valley are interpolated. The fringe depth is measured by subtraction of the fitted valleys from the fitted peaks; and finally, the mean and standard deviation of the fringe depth are calculated. The results of all the optofluidic chips with MZI and homodyne detection is presented in table 5.2 A second MATLAB script is used to measure the fringe spacing between the nulls. A linear relation was observed between fringe spacing and wavelength. Therefore, the fringe spacing at $940 \mathrm{~nm}$ and $1040 \mathrm{~nm}$ could be calculated using a linear fit of the fringe spacing against wavelength (for more details, see appendix B). The geometric path length difference from the fringe spacing at $940 \mathrm{~nm}$ and $1040 \mathrm{~nm}$ should result in the same geometric path length difference if the cross-sections of the fabricated chips are exactly as simulated. The null depth is plotted in figure 5.15 against the different calculated geometric path length differences. The error bar in the null depth represents the standard deviation of 200 spectra and the error bar in the geometric path length denotes differences between the calculated geometric path length from the fringe spacing at $940 \mathrm{~nm}$ and $1040 \mathrm{~nm}$.

\subsubsection{Results}

The output power of the SLD was measured to be $1.2 \mathrm{~mW}$ and $5.7 \mathrm{~mW}$ for the low-, and high power mode setting, respectively. The high power setting was used. the output has a $92.2 \mathrm{~nm}$ bandwidth at a central wavelength of $994.2 \mathrm{~nm}$. Table 5.2 (on page 112) shows the analysis on the design 3 chips. The MZI signal is successfully mixed with the local oscillator (LO) as can be seen from the integration times and figure 5.14. The path of the buried LO has a similar length as the sensing path and half of the light intensity is in the LO path, while the other half of the light intensity is in the MZI path. Theoretically, potential conclusions could be drawn from the relative losses of the sensing and buried waveguides without having to consider the coupling losses. Unfortunately, a wide range of integration time ratios was determined between the MZI- (25\% sensing path) and 


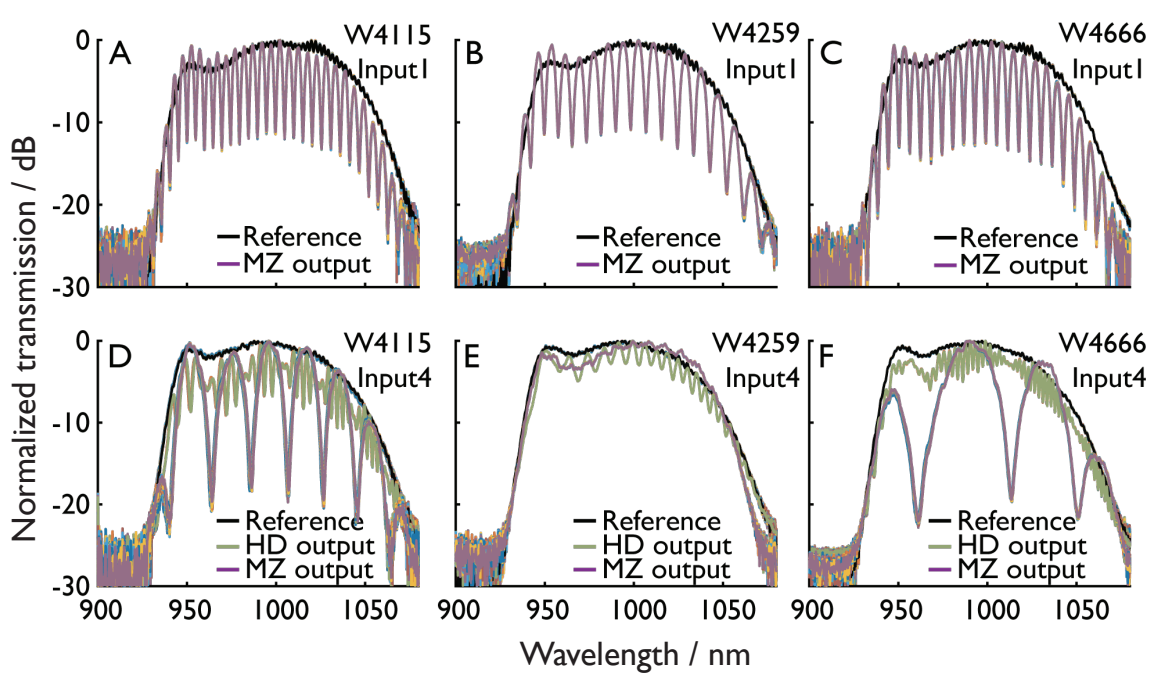

Figure 5.14: Optical response recorded for two different photonic integrated circuits (MZI- and homodyne lay-out) against wavelength for the three wafers 4115, 4259 and 4666. Subplots A, B and $\mathrm{C}$ present data recorded from simple MZI with 10 fringes designed in the spectral window. In subplot $\mathrm{D}, \mathrm{E}$ and $\mathrm{F}$ again data is presented that has been recorded for a simple MZI designed to have complete destructive interference. Half of the light intensity of the output of the MZI is recorded, and the other halve is recombined with the local oscillator to realize the homodyne (HD) output.

homodyne interferometer ( $25 \%$ sensing path $+25 \%$ buried path) showed a wide range indicating the propagation losses (likely the sensing paths) to vary from chip to chip.

Figure 5.14 shows the data from the 3 fabricated chips of design 32 . The normalized output of the MZI and homodyne sensors $\left(\mathrm{D}_{32}\right)$ are presented. The spectra show single mode operation (only one modulation frequency). The stray light was responsible for the observed spectral features (a modulation independent on the ion-concentration) on the reference spectra. Large variations in fringe spacing is observe on the same optical design between the wafer W4259, and wafers W4115 \& W466. Small variation in cross-section (with the long arms of the MZI) would explain these large deviations.

A large spread in the number of spectral fringes can be seen in figure 5.15. Because the sensing arms are rather long $(5 \mathrm{~cm})$, small changes in the core size of the $\mathrm{Si}_{3} \mathrm{~N}_{4}$ core result in large deviations from design spectral output. Still, high fringe depths were recorded close to what is theoretically obtainable with the $0.6 \mathrm{~nm}$ resolution recording devices (see Appendix B).

The fringe spacing at $940 \mathrm{~nm}$ and $1040 \mathrm{~nm}$ decreases and increases nonlinearly with the concentration of $\mathrm{Na}_{2} \mathrm{SO}_{4}$ as shown in figure 5.16 for designs D31 \& D32, and D33, respectively. Hereby the change in the real part of the refractive index due to the ionic content in water is determined. The null depth shows the effect of the optical path difference and the power imbalance between the two MZ arms. A smaller change in null depth was expected based on the calculations presented in appendix B. The change in complex refractive index can be recovered by fitting of the spectral data. But, since this 


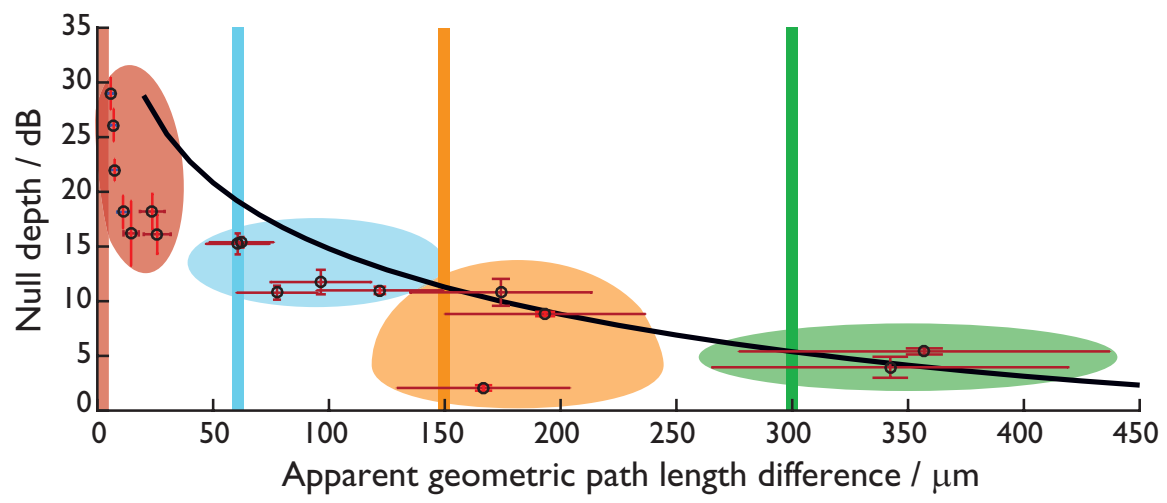

Figure 5.15: The performance of all the chips based on a MZI with Y-junctions design. The black solid line is the expected null depth from a recording device with a resolution of $0.6 \mathrm{~nm}$. The vertical colored red, blue, orange, and green lines represent MZI with an OPD of $0.3 \mu \mathrm{m}, 59.5 \mu \mathrm{m}$, $150 \mu \mathrm{m}$ and $300 \mu \mathrm{m}$, respectively. The data points from the different designs are outlined with a colored circle.

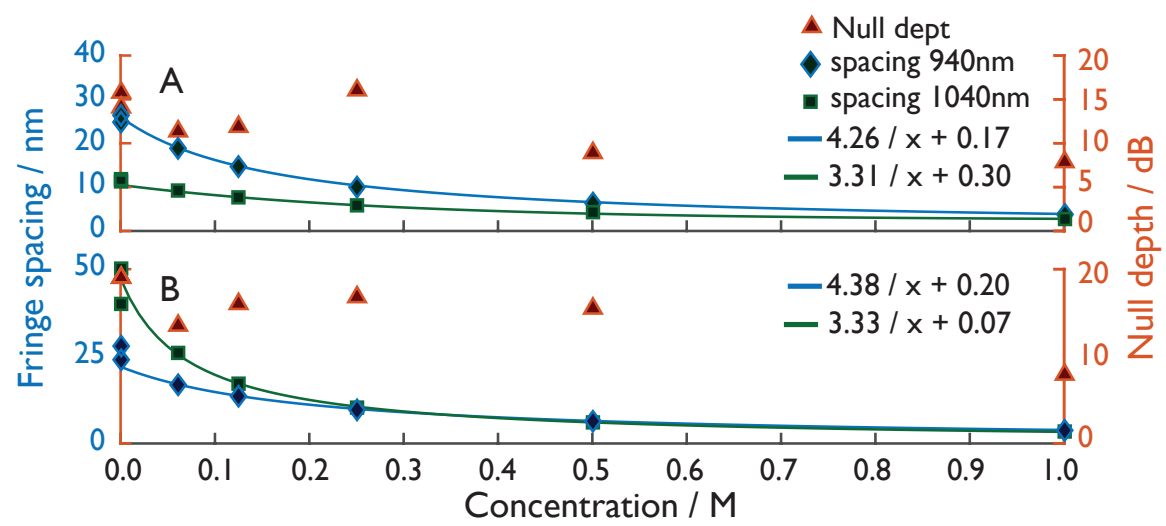

Figure 5.16: The shift of fringes and change in null depth for different concentrations of $\mathrm{Na}_{2} \mathrm{SO}_{4}$. The analyzed data of two on-chip MZI were intended to have 10 spectral fringes (A and B).

null depth is dominated by the imbalance of refractive index, the salt concentration/salt type cannot be determined without proper fitting the data. 
Table 5.2: Summary of data recorded with the interferometers on-chip

\begin{tabular}{|c|c|c|c|c|c|}
\hline $\begin{array}{c}\text { Wafer } \\
\text { nr. }\end{array}$ & $\begin{array}{l}\text { Input } \\
\text { (output) }\end{array}$ & $\begin{array}{c}\text { Chip } \\
\text { Design }\end{array}$ & $\begin{array}{c}\text { Integration } \\
\text { time (ms) }\end{array}$ & $\begin{array}{c}\text { Fringe Depth } \\
\text { Average } \pm S D\end{array}$ & $\begin{array}{c}\text { Nr. } \\
\text { Fringes }\end{array}$ \\
\hline \multirow[t]{3}{*}{4115} & $1(\mathrm{MZI})$ & D31 & 20.226 & $<4$ & n.d.** \\
\hline & 4 (HD) & & 12.195 & $9.20 \pm 1.61$ & 5 \\
\hline & $4(\mathrm{MZI})$ & & 110.396 & $26.1 \pm 2$ & 1 \\
\hline \multirow[t]{3}{*}{4666} & 1 (MZI) & & 52.482 & $16.12 \pm 1.79$ & 7 \\
\hline & $4(\mathrm{HD})$ & & 27.524 & $6.18 \pm 0.91$ & 15 \\
\hline & $4(\mathrm{MZI})$ & & 243.623 & $22 \pm 2$ & 2 \\
\hline \multirow[t]{3}{*}{4115} & 1 (MZI) & D32 & 1000 & $10.99 \pm 0.34$ & 20 \\
\hline & 4 (HD) & & 21.272 & $5.61 \pm 0.93$ & 25 \\
\hline & $4(\mathrm{MZI})$ & & 902.773 & $18.20 \pm 1.61$ & 5 \\
\hline \multirow[t]{3}{*}{4259} & 1 (MZI) & & 70 & $10.78 \pm 0.64$ & 13 \\
\hline & $4(\mathrm{HD})$ & & 4.028 & $<4$ & n.d. \\
\hline & 4 (MZI) & & 172.938 & $<4$ & n.d. \\
\hline \multirow[t]{3}{*}{4666} & 1 (MZI) & & 900.441 & $11.75 \pm 1.12$ & 17 \\
\hline & 4 (HD) & & 3.079 & $4.17 \pm 1.12$ & 35 \\
\hline & $4(\mathrm{MZI})$ & & 48.879 & $18.15 \pm 1.50$ & 3 \\
\hline \multirow[t]{3}{*}{4115} & 1 (MZI) & D33 & 267.21 & $8.83 \pm 0.20$ & 31 \\
\hline & $4(\mathrm{HD})$ & & 6.315 & $4.22 \pm 0.44$ & 61 \\
\hline & 4 (MZI) & & 31.927 & $16.20 \pm 2.96$ & 5 \\
\hline \multirow[t]{3}{*}{4259} & 1 (MZI) & & 1000 & $10.81 \pm 1.23$ & 28 \\
\hline & 4 (HD) & & 6.315 & $<4$ & n.d. \\
\hline & $4(\mathrm{MZI})$ & & 31.927 & $29 \pm 2$ & 1 \\
\hline \multirow[t]{3}{*}{4666} & 1 (MZI) & & 470.373 & $<4$ & n.d. \\
\hline & 4 (HD) & & 12.894 & $<4$ & n.d. \\
\hline & 4 (MZI) & & 2.064 & $<4$ & n.d. \\
\hline \multirow[t]{3}{*}{4115} & 1 (MZI) & D34 & 1000 & $4.59 \pm 0.83 *$ & 53 \\
\hline & 4 (HD) & & 7.61 & $5.40 \pm 0.88$ & 44 \\
\hline & 4 (MZI) & & 40.581 & $15.24 \pm 0.96$ & 9 \\
\hline \multirow[t]{3}{*}{4259} & 1 (MZI) & & 235.132 & $5.41 \pm 0.28 *$ & 56 \\
\hline & $4(\mathrm{HD})$ & & 0.5087 & $5.67 \pm 0.69$ & 43 \\
\hline & $4(\mathrm{MZI})$ & & 2.064 & $15.39 \pm 0.48$ & 9 \\
\hline
\end{tabular}




\subsection{Conclusion}

Multiple approaches to measuring differential transmission caused by the presence of ions in water have been presented. They may also be applied to different applications where small differences in absorption need to be measured between fluids of similar composition. It is feasible to design and simulate a differential absorption based optofluidic sensor for determination of ionic content in water. The parameters leading to a $100 \mathrm{~nm}$ single mode wavelength region with bend losses lower than $0.02 \mathrm{~dB} \mathrm{~cm}^{-1}$ and sensitivity around $20 \%$ whereas keeping the reasonable foundry tolerances were presented.

Moreover, the optimal sensing arm length was found to be independent of the concentration of ionic content due to small differences in transmitted intensity but does depend on the losses of the waveguides. Adjustments of the sensing length can be designed to compensate for these losses. Interferometric techniques permit determination of the differential complex refractive index between two fluids and can be used to measure differential absorption signal on-chip. The adiabatic $\Xi$ coupler with its flattened spectral response could be an interesting tool for interferometric designs as has been shown for a Michelson interferometer.

Initial results show the improved design led to the desired single mode behavior and reduced losses. High contrast in visibility was observed with a highest null depth of $(29 \pm 2) \mathrm{dB}$. The experiments with $\mathrm{Na}_{2} \mathrm{SO}_{4}$ showed the sensors to be sensitive to the real and imaginair part of the refractive index changes caused by the ionic content in water. Further work will involve the interferometric data fitting of the spectral output recorded for the different designs with increasing concentrations of ionic species in the fluidic channel. 



\section{Bibliography}

[1] P. Fletcher, "World within a world." Accessed: 14.07.2017. — p.94.

[2] J. A. Plant, N. Voulvoulis, and K. V. Ragnarsdottir, Pollutants, human health and the environment: a risk based approach. John Wiley \& Sons, 2012. — p.96.

[3] P. H. Gleick, "Water and terrorism," Water policy, vol. 8, no. 6, pp. 481-503, 2006. - p.96.

[4] W. D. W. Q. Committee, "Water Sampling and Analysis," Guidelines for drinking water quality, vol. 4th ed., pp. 51-72, 2011. - p.96.

[5] J. P. Murrihy, M. C. Breadmore, A. Tan, M. McEnery, J. Alderman, C. O’Mathuna, A. P. O'Neill, P. O'Brien, N. Advoldvic, P. R. Haddad, and J. D. Glennon, "Ion chromatography on-chip," Journal of Chromatography A, vol. 924, no. 1, pp. 233 238, 2001. 14th International Symposium on Microscale Separations and Analysis. - p.96.

[6] J. Raich, "Review of sensors to monitor water quality," European Commission Joint Research Centre, JRC85442, 2013. - p.96.

[7] R. Suhrmann and F. Breyer, "Untersuchungen im ultraroten absorptionsspektrum über die Änderung des lösungsmittels durch die gelöste substanz. i. uber den einfluss gelöster salze auf den assoziationszustand des löstenden wassers," Z. Physik. Chem. B, vol. 20, no. 17, pp. 17-53, 1933. - p.96.

[8] G. R. Choppin and K. Buijs, "Near-infrared studies of the structure of water. ii. ionic solutions," The Journal of Chemical Physics, vol. 39, no. 8, pp. 2042-2050, 1963. - p..

[9] J. Lin, J. Zhou, and C. W. Brown, "Identification of Electrolytes in Aqueous Solutions from Near-IR Spectra," Applied Spectroscopy, vol. 50, pp. 444-448, 1996. - p..

[10] J. Lin and C. W. Brown, "Spectroscopic measurement of $\mathrm{NaCl}$ and seawater salinity in the near-IR region of 680-1230 nm," Applied Spectroscopy, vol. 47, pp. 239-241, 1993. - p.96.

[11] G. W. Steen, E. C. Fuchs, A. D. Wexler, and H. L. Offerhaus, "Identification and quantification of 16 inorganic ions in water by gaussian curve fitting of near-infrared difference absorbance spectra," Appl. Opt., vol. 54, pp. 5937-5942, Jul 2015. — p.97.

[12] G. Steen, A. D. Wexler, and H. L. Offerhaus, "Optofluidic interferometry chip designs of differential nir absorbance based sensors for identification and quantification of electrolytes," in Advanced Photonics 2016 (IPR, NOMA, Sensors, Networks, SPPCom, SOF), p. SeW3D.2, Optical Society of America, 2016. — p.97.

[13] A. Beer, "Bestimmung der absorption des rothen lichts in farbigen flüssigkeiten," Annalen der Physik, vol. 162, no. 5, pp. 78-88, 1852. - p.101. 
[14] L. Zhuang, D. Marpaung, M. Burla, W. Beeker, A. Leinse, and C. Roeloffzen, "Low-loss, high-index-contrast si3n4/sio2 optical waveguides for optical delay lines in microwave photonics signal processing," Opt. Express, vol. 19, pp. 23162-23170, Nov 2011. - p.102.

[15] K. Wallace, G. Hardy, and E. Serabyn, "Deep and stable interferometric nulling of broadband light with implications for observing planets around nearby stars," Nature, vol. 406, no. 6797, pp. 700-702, 2000. - p.103.

[16] M. R. Bolcar and R. G. Lyon, "Approaches for achieving broadband achromatic phase shifts for visible nulling coronagraphy," 2012. - p.103.

[17] P. Hlubina, M. Kadulová, and D. Ciprian, "Spectral interferometry-based chromatic dispersion measurement of fibre including the zero-dispersion wavelength," Journal of the European Optical Society - Rapid publications, vol. 7, no. 0, 2012. - p.104.

[18] B. Anderson, E. Bernhardt, and M. G. Kuzyk, "A white light interferometric microscope for measuring dose-dependent reversible photodegradation," Journal of Applied Physics, vol. 114, no. 12, p. 123103, 2013. - p.104.

[19] H. K. Hsiao, K. A. Winick, J. D. Monnier, and J. P. Berger, "Integrated optic beam combiners for stellar interferometry and nulling at near- and mid-infrared wavelengths," 2010. - p.106. 



\section{CHAPTER}

\section{Summary and outlook}

66 He used often to say there was only one Road; that it was like a great river: its springs were at every doorstep, and every path was its tributary

J.R.R. Tolkien, 


\subsection{Reflections}

This work is like a narrow shallow side part of a big river. The fast development of the sensor industry is pushed by the contribution of many small innovative ideas. This work in itself might not have a big influence, however when it returns to the main flow it might prove useful in developing better real-time detectors to improved our daily life.

The purpose of the work was to design and realize an integrated optofluidic sensor that can be utilized to detect inorganic ions (in water) in-/online and real-time. The study of 16 single electrolytes described in chapter 2 showed the wavelength range of $0.94 \mu \mathrm{m}$ to $1.04 \mu \mathrm{m}$ to be suitable for identification and quantification of single electrolytes. Furthermore, this study showed a commercial photo-spectrometer to be functional in a concentration range of $30 \mathrm{mM}$ to $500 \mathrm{mM}$. Also, color coding data in a $2 \mathrm{D}$ plot proved to be powerful tool to compare large amounts of spectra easily. Finally, two analysis strategies, i.e., frequency-wise linear regression (FWLR) fitting and Gaussian curves fitting were applied to the spectral data. The spectral signature of each electrolyte was determined from the FWLR analysis. Truth tables can be made from the Gaussian curves analysis to process spectral data to identify and quantify single electrolytes.

The second study (chapter 3) expanded on the experimental framework by examining the role of temperature in de-mixing of absorbance spectra measured in mixed aqueous $\mathrm{Na}_{2} \mathrm{SO}_{4}$ and $\mathrm{NaNO}_{3}$ solutions. First, the influence of temperature on the absorbance spectrum of demineralized water was determined. Second, the absorbance spectra of five separate electrolytes $\left(\mathrm{NaNO}_{2}, \mathrm{NaNO}_{3}, \mathrm{CaCl}_{2}, \mathrm{~K}_{2} \mathrm{CO}_{3}\right.$, and $\left.\mathrm{NaOH}\right)$ at three temperatures $\left(4^{\circ} \mathrm{C}, 25^{\circ} \mathrm{C}\right.$ and $50^{\circ} \mathrm{C}$ ) for concentrations ranging from $0.0625 \mathrm{M}$ to $0.5 \mathrm{M}$ were examined. These five electrolytes showed similar temperature dependencies. Finally, the absorbance spectra of mixed solutions were investigated at temperatures $5{ }^{\circ} \mathrm{C}, 15^{\circ} \mathrm{C}$, $25^{\circ} \mathrm{C}, 35^{\circ} \mathrm{C}$ and $45^{\circ} \mathrm{C}$ for concentrations ranging from $0.0625 \mathrm{M}$ to $0.5 \mathrm{M}$ per electrolyte in the mixture. The near infrared (NIR) spectral window from $650 \mathrm{~nm}$ to $1100 \mathrm{~nm}$ was recorded to observe the ionic and temperature influences on the vibrational modes of the $\mathrm{OH}$ bond in the solvent molecules. The effects of dissolving $\mathrm{Na}_{2} \mathrm{SO}_{4}$ and $\mathrm{NaNO}_{3}$ were found to be nonlinearly cumulative at lower temperatures indicating extended alteration of the water structure beyond the first hydration shell. A similar trend was observed for a mixture of $\mathrm{Na}_{2} \mathrm{CO}_{3}$ and $\mathrm{NaCl}$. Furthermore, higher temperatures are suited for recovering the separate component absorption signatures of an electrolyte mixture. The near-infrared spectral regime is well suited for integrated sensing, and therefore these results laid the foundation to design an integrated sensor based on NIR absorption spectrscopy to identify inorganic species in water.

Single mode operation in combination with low propagation- and bend losses is required to realized such an approach. The cross-section of TriPleX waveguides was optimized for the NIR spectral region that was established with first studies. The size of the $\mathrm{Si}_{3} \mathrm{~N}_{4}$ core (for the buried and the sensing structures) was optimized based on single mode operation, and a balance between the sensitivity and the losses. Ten optical layouts were designed. The wafer mask based on the designs was fabricated by LioniX and used to realize five wafers in a dedicated wafer run. Each wafer was diced to produce a total of 90 optofluidic chips. Chapter 4 discussed the sensors that were realized in the first fabrication run. A chip yield of $(38.5 \%)$ was determined using microscopic inspection. The quality of the top-cladding led to the lower chip yield ( $\sim 70 \%$ was expected). An 
improper edge of the top-cladding further caused strong scatter points. From these chips multiple lessons were learned that proved vital in the next fabrication run. The results from the first run led to a further improvement of the TriPleX cross-section to avoid the multimode behavior.

Chapter 5 presents the further development with additional optimization for fabrication tolerances. Multiple integrated photonic circuit designs varying from simple electronic subtraction to interferometric detection were reviewed. Single mode operation was observed for the chips produced in the second fabrication run. Similar losses in the $5 \mathrm{~cm}$ sensing spirals arms were found, as evidenced by the deep null depth (as high as $(29 \pm 2) \mathrm{dB})$. The experiments with $\mathrm{Na}_{2} \mathrm{SO}_{4}$ showed the sensors to be sensitive to the real and imaginaire part of the refractive index changes caused by the ionic content in water.

\subsection{Future perspective}

The losses of the optofluidic chips needs to be characterized to realize a point of care device, such as the propagation- (buried- and sensing waveguides), insertion-, bending-, and interfaces losses (between buried- and sensing structure). Also the fitting of the spectral data is relevant to extract the real and imaginary part of the refractive index change due to the ionic content in water.

During one of the experiments with an MZI layout a small bubble (mm size) was located on top of the sensing spiral. After the chip holder was rotated spectral shifting of the fringes and intensity change were observed. Thus the setup needs to be improved to include some form of a degasser. Also the SNR needs to be further improved by

- bonding the fiberarry to the chip

- thermal control of the chip

- integration of the lightsource and detectors. e.g., an array of VCSELS on a hybrid chip.

- a concentration step of the sample. The micro fluidic window requires only small amount of sample volume, therefore the heat required to concentrate a sample might be acceptable.

A temperature modulation can be used to calibrate the sensor. The small sampling volume of an optofluidic sensor makes fast temperature control possible at different temperatures, however the temperature modulation for usable signal strength needs to be $50{ }^{\circ} \mathrm{C}$ (see chapter 3 ).

It would be interesting to observe whether or not arsenic- and mercury ions have influence on the absorbance spectrum of water. Hydrocarbons, acids, alcohols and sugars are expected to have their own unique spectral fringerprint on the water absorbance spectrum and it could be very interesting for process control in (biological) waste and industrial water treatment. Furthermore some evidence was found that pesticides (Alachlor and 
Atrazine) have an influence on the $\mathrm{OH}$ bonds. ${ }^{1}$ Some other potential lower hanging fruit for the absorbance based optofluidic sensors is complex metal ions. Most of these ions have a direct absorbance in the visible region of the electromagnetic spectrum, due to their 3d transitions). An in- \& online sensor can be fabricated using the TriPleX platform using the design consideration presented in chapter 4 and 5 to realize a broadband single mode operation in the visible (VIS) spectral region. A combination of VIS and NIR spectroscopy could make a robust sensor.

\footnotetext{
${ }^{1}$ Gowen, A. A., Tsuchisaka, Y., O’Donnell, C., \& Tsenkova, R. (2011). Investigation of the potential of near infrared spectroscopy for the detection and quantification of pesticides in aqueous solution. American Journal of Analytical Chemistry, 2(08), 53.
} 

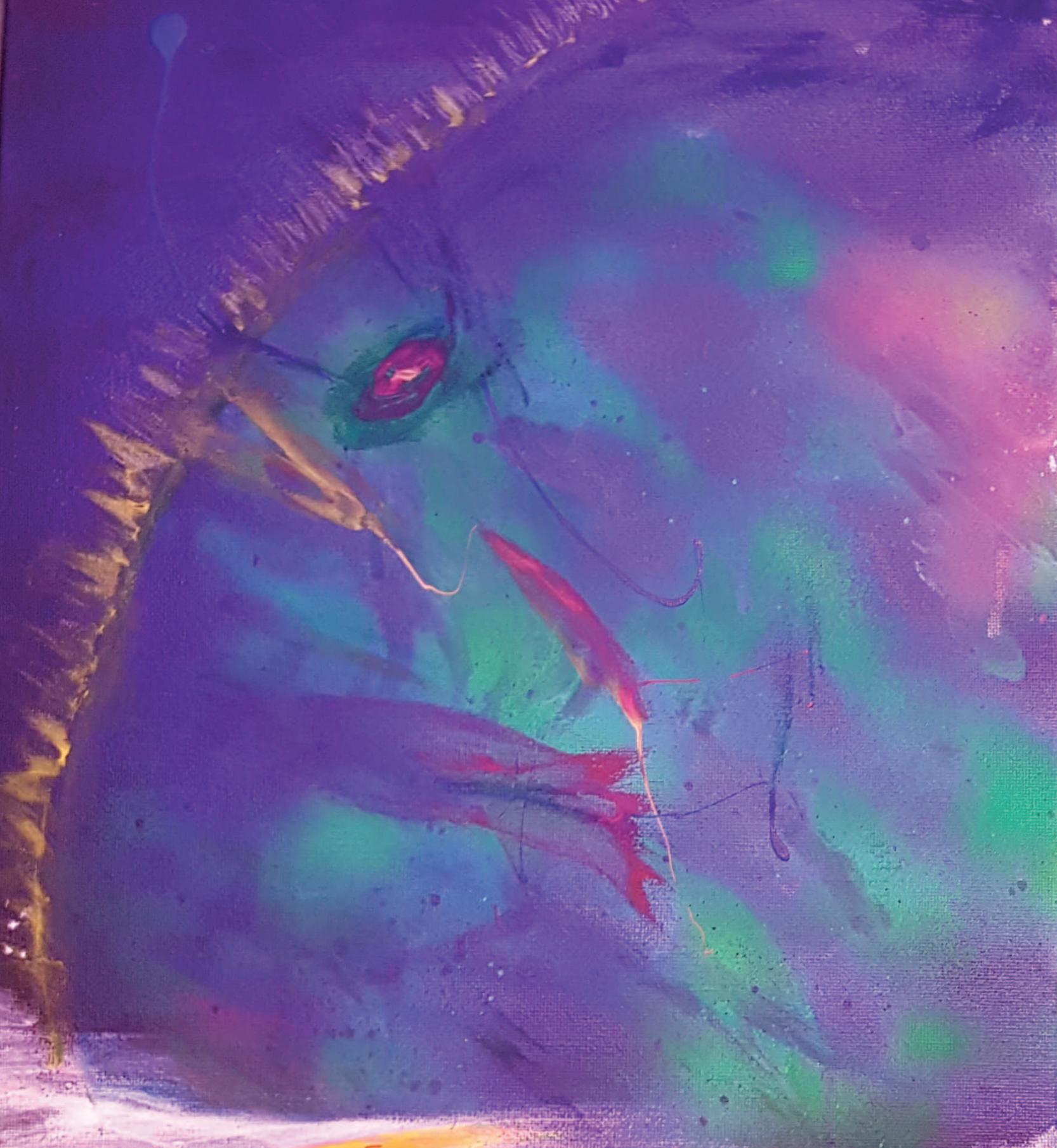

.

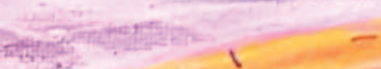

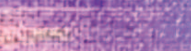


-250 in

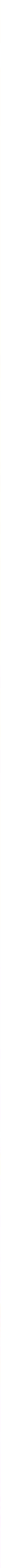




\section{APPENDIX}

\section{Supplemental Figures - Chapter 3}

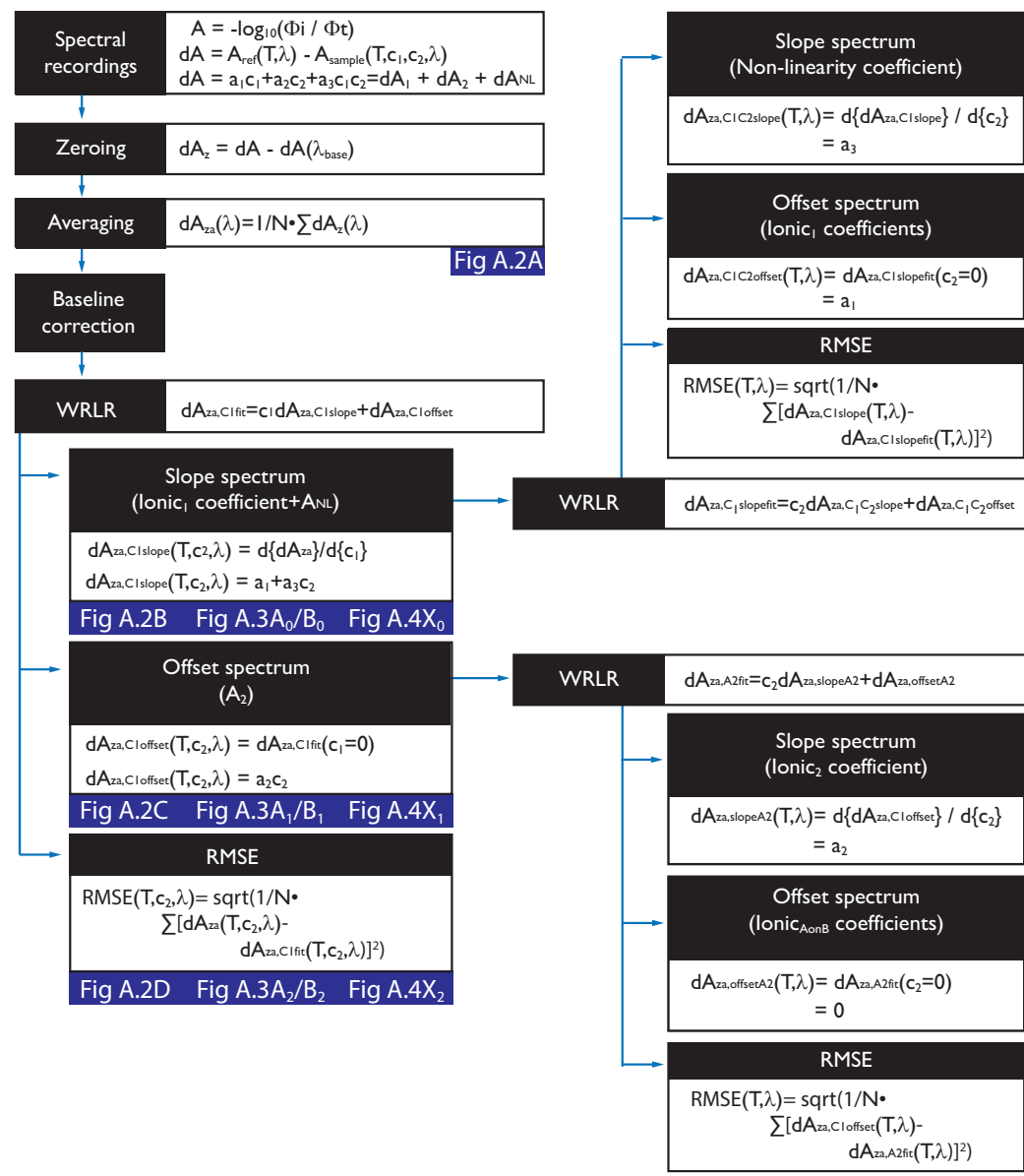

Figure A.1: The diagram shows the data processing algorithm with outputs presented in this appendix. 


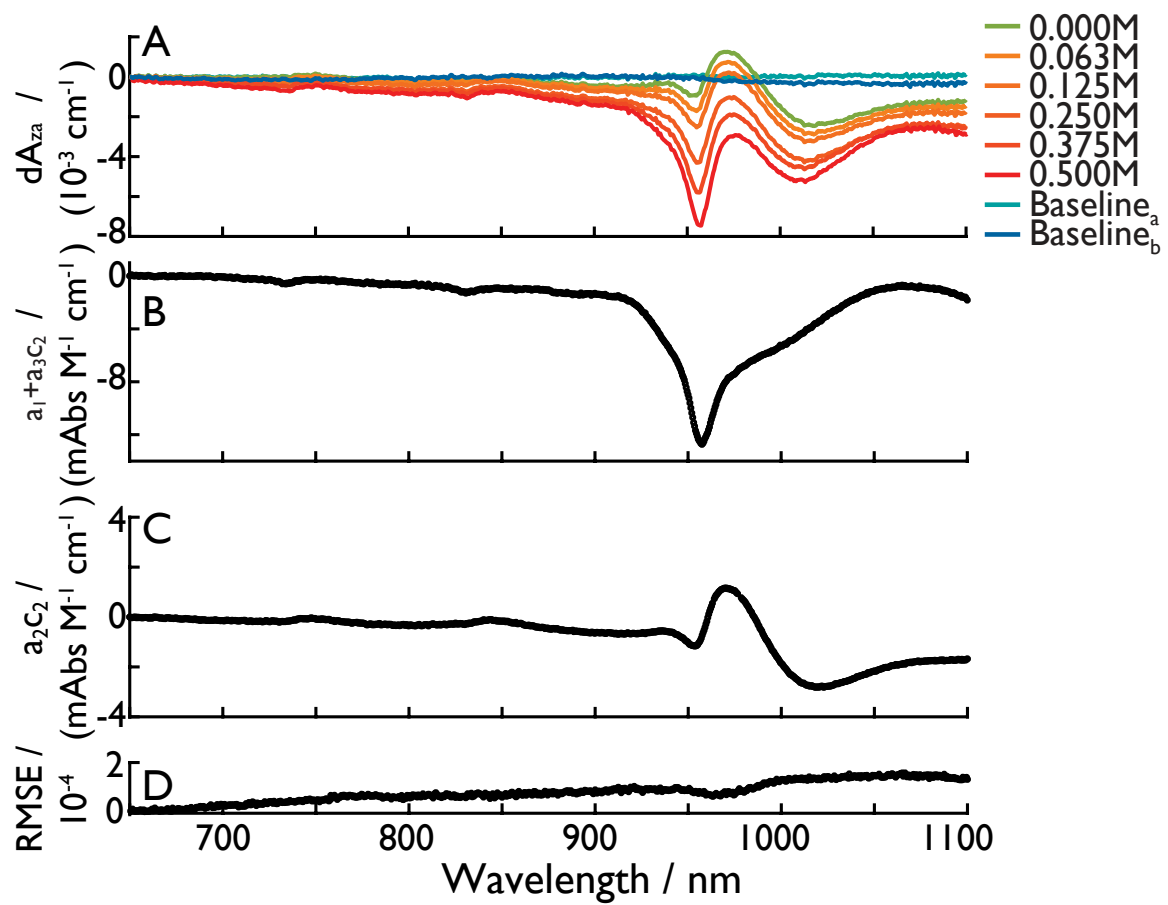

Figure A.2: A typical data set is shown of a $\mathrm{Na}_{2} \mathrm{SO}_{4} / \mathrm{NaNO}_{3}$ mixture. The recordings (A) shows the influence $0.375 \mathrm{M} \mathrm{NaNO}_{3}$ dissolved in water and varying the dissolved $\mathrm{Na}_{2} \mathrm{SO}_{4}$ concentration on the absorbance spectrum of water. The contribution of the varying $\mathrm{Na}_{2} \mathrm{SO}_{4}$ and nonlinear term (B) are extracted by the WRLR analysis. The offset values (C) gives the spectral signature due to the constant electrolyte in the mixture $\left(\mathrm{NaNO}_{3}, 0.375 \mathrm{M}\right)$. The RMSE values (D) give the goodness of the fit. 

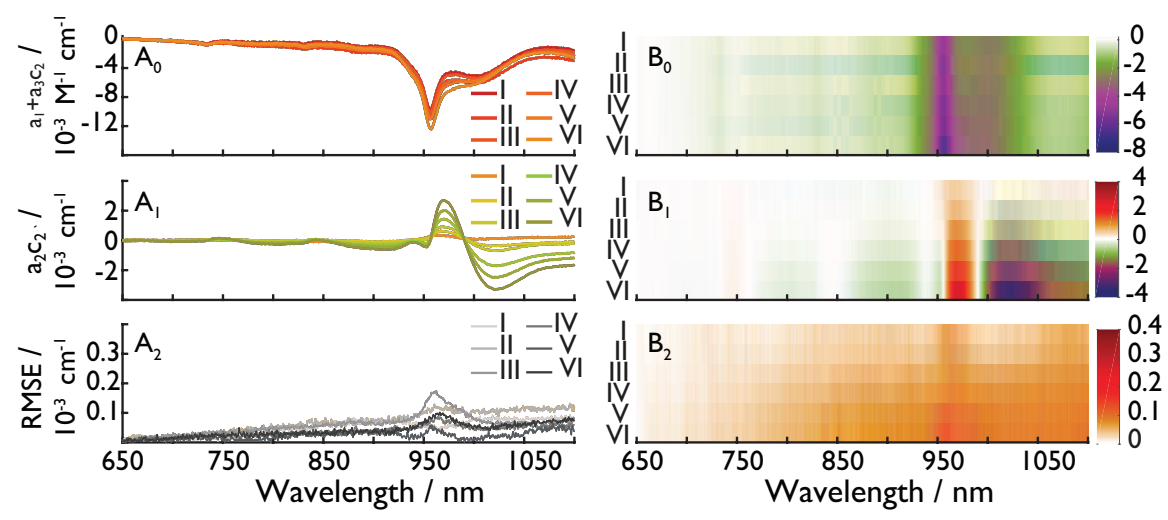

Figure A.3: The spectral slope $\left(\mathrm{A}_{0}\right)$, offset $\left(\mathrm{A}_{1}\right)$ and $\mathrm{RMSE}\left(\mathrm{A}_{2}\right)$ values of the WRLR analysis are presented. Line plots I, II, III, IV, V, and VI show the analyzed data sets recorded at $25^{\circ} \mathrm{C}$ with varying $\mathrm{Na}_{2} \mathrm{SO}_{4}(0 \mathrm{M}$ to $0.5 \mathrm{M})$ and a $\mathrm{NaNO}_{3}$ concentration of $0 \mathrm{M}, 0.063 \mathrm{M}, 0.125 \mathrm{M}, 0.250 \mathrm{M}$, $0.375 \mathrm{M}$ and $0.5 \mathrm{M}$, respectively. The values of the spectral slope $\left(\mathrm{B}_{0}\right)$, offset $\left(\mathrm{B}_{1}\right)$ and $\operatorname{RSME}\left(\mathrm{B}_{2}\right)$ are color-coded and stacked on top of each other.

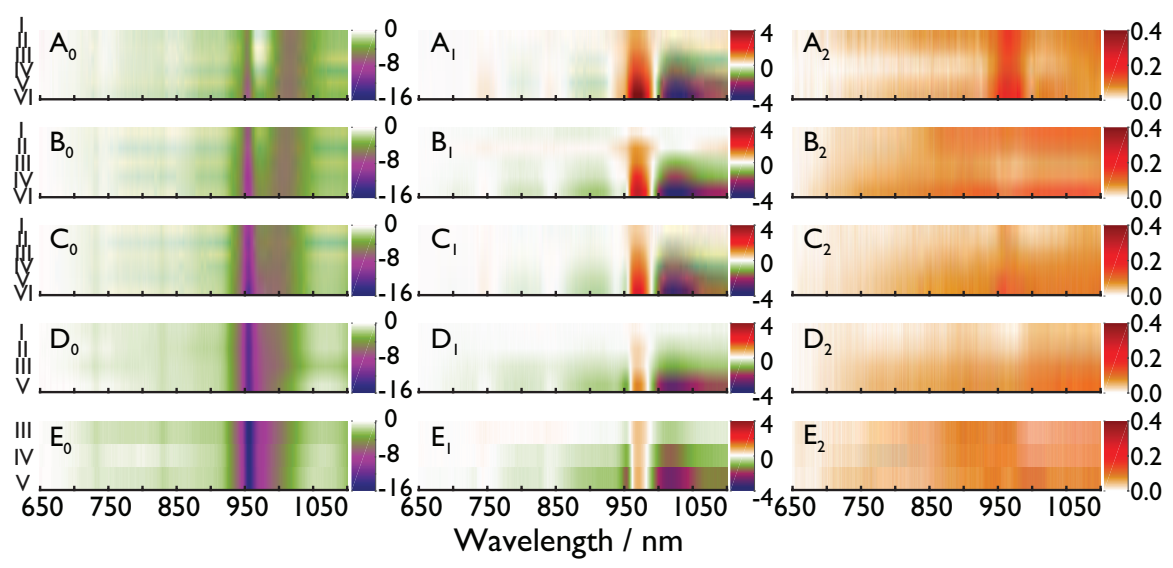

Figure A.4: The WRLR analyses of the mixtures recorded at $5{ }^{\circ} \mathrm{C}, 15^{\circ} \mathrm{C}, 25^{\circ} \mathrm{C}, 35^{\circ} \mathrm{C}$ and $45^{\circ} \mathrm{C}$ are presented in subplots $A_{x}, B_{x}, C_{x}, D_{x}$, and $E_{x}$, respectively. The slope-, offset-, and RMSE values are presented in subfigures $X_{0}, X_{1}$, and $X_{2}$, respectively. The color map is the same in all subfigures to better visualize patterns in the data. The results of the WRLR analyses are stacked on top of each other. I, II, III, IV, V, and VI represent the stacked fitted 2D color-coded data for a varying $\mathrm{Na}_{2} \mathrm{SO}_{4}(0 \mathrm{M}$ to $0.5 \mathrm{M})$ and a $\mathrm{NaNO}_{3}$ concentration $0 \mathrm{M}, 0.063 \mathrm{M}, 0.125 \mathrm{M}, 0.250 \mathrm{M}$, $0.375 \mathrm{M}$ and $0.500 \mathrm{M}$, respectively. 



\section{APPENDIX \\ Interferometric data fitting}

\section{B.1 Broadband Mach Zehnder}

In a Mach Zehnder setup the spectral power density $S$ generated by a light source is bisected by an optical component. Bulk optical components (e.g., cube/plate, pellicle and dichroic beamsplitter) can be used or integrated optical components (e.g., y-splitters, bidirectional-, $\Xi$ - and MMI couplers) can be utilized to split the light into two propagating beams with spectral power densities $S_{0}$ and $S_{1}$. One path is typically used to probe light-matter interaction while the other path remains constant. The spectral intensity of recombined light depends on the phase- and intensity relation between the two beams. The interference between the two beams at angular frequency $(\omega)$ is expressed by the spectral interference law ${ }^{1}$ :

$$
\begin{aligned}
S_{M Z}\left(\boldsymbol{R}, \Delta_{M Z} ; \omega\right) & =S_{0}(\boldsymbol{R} ; \omega)+S_{1}(\boldsymbol{R} ; \omega) \\
& +2 \sqrt{S_{0}(\boldsymbol{R} ; \omega) S_{1}(\boldsymbol{R} ; \omega)} \cos \left(\frac{\omega}{c} \Delta_{M Z}(\boldsymbol{R} ; \omega)\right) .
\end{aligned}
$$

The $\mathbf{R}$ in the spectral inference law is the transverse position vector, $S_{M Z}$ is the resultant spectral power density and $\Delta_{M Z}$ is the spatially dependent optical path difference between the two propagating beams at the output of the Mach Zehnder inteferometer. The optical power at the output of the Mach Zehnder interferometer can be directly guided by free space components to a device capable of recording the spectral power, or an optical fibre can be used to couple the light from the output of the interferometer to a spectrometer. The effect of a wavelength-dependent aperture function $(A(\boldsymbol{R} ; \lambda))$ due to the numerical aperture of the fiber and a response function $\left(R\left(\lambda-\lambda^{\prime}\right)\right)$ of the spectrometer can be expressed as

$$
I_{M Z}\left(\Delta_{M Z} ; \omega\right)=\iint S_{M Z}\left(\boldsymbol{R}, \Delta_{M Z} ; \omega^{\prime}\right) A\left(\boldsymbol{R} ; \omega^{\prime}\right) R\left(\omega-\omega^{\prime}\right) d^{2} \boldsymbol{R} d \omega^{\prime}
$$

\footnotetext{
${ }^{1}$ Hlubina, P. (2003, November). Dispersive white-light spectral two-beam interference under general measurement conditions. In 13th Polish-Czech-Slovak Conference on Wave and Quantum Aspects of Contemporary Optics (Vol. 5259, pp. 281-289). International Society for Optics and Photonics.
} 
Equation B.1 and B.2 can be combined by assuming the response function of the spectrometer to be a Gaussion function with half-width $\Gamma_{R}$, i.e.,

$$
R\left(\omega-\omega^{\prime}\right)=\frac{R_{0}}{\sqrt{\pi} \Gamma_{R}} e^{-\frac{\left(\omega-\omega^{\prime}\right)^{2}}{\Gamma_{R}^{2}}}
$$

and further assuming the spectral phase $\left(\Phi(\omega)=\frac{c}{\omega} \Delta_{M Z}(\omega)\right)$ can be expanded in a second order Taylor series

$$
\Phi\left(\omega^{\prime}\right) \approx \Phi(\omega)+\Phi^{\prime}(\omega)\left(\omega^{\prime}-\omega\right)+0.5 \Phi^{\prime \prime}(\omega)\left(\omega^{\prime}-\omega\right)^{2}
$$

and finally assuming the functions $S_{0}(\boldsymbol{R} ; \omega), S_{1}(\boldsymbol{R} ; \omega)$ and $A(\boldsymbol{R} ; \omega)$ to vary slowly with the wavelength,

$$
\begin{aligned}
S_{M Z}(\omega) & =I_{0}(\omega)+I_{1}(\omega)+2 V_{A}(\omega) V_{R}(\omega) \sqrt{I_{0}(\omega) I_{1}(\omega)} \cos \left(\frac{\omega}{c} \Delta_{M Z}(\omega)\right), \\
& =I_{(i n)}(\omega)\left(1+V_{I}(\omega)\right) \cos \left(\Phi_{R}\right) .
\end{aligned}
$$

$V_{A}$ and $V_{R}$ are spectral visibility terms of the spectral fringes. High visibility ( $V$ approaching unity) leads to a nearly $100 \%$ fringe modulation. The overall visibility $\left(V_{I}\right)$ of the spectral fringes depends on dispersion, the response function of the recording device, coherence length of the light source and the power imbalance of beams of the reference and sample arm in the Mach Zehnder: $V_{I}=V_{A} V_{R} V_{P} . V_{A}$ is the visibility of the spectral interference fringes at the output of the fiber used to collect the light at one output port of the Mach Zehnder interferometer. It also includes the effect of mode overlap. The visibility due to the power imbalance $\left(V_{P}\right)$ and the visibility due to the effect of limited spectral resolution of a spectrometer $\left(V_{R}\right)$ and the spectral phase $\Phi_{R}$ are given by:

$$
\begin{aligned}
V_{P} & =2 \frac{\sqrt{I_{0} I_{1}}}{I_{0}+I_{1}} \\
\text { and } & \\
V_{R}(\omega) & =\frac{1}{\sqrt{1+\varrho^{2}}} e^{-\frac{1}{4} \Gamma_{R}^{2} \Phi^{\prime 2} \frac{1}{1+\varrho^{2}}} \\
\text { and } & \\
\Phi_{R}(\omega) & =\Phi(\omega)+\frac{1}{4} \Gamma_{R}^{2} \Phi^{\prime 2}(\omega) \frac{\varrho(\omega)}{1+\varrho^{2}(\omega)}-\frac{1}{2} \arctan (\varrho(\omega))=\Phi(\omega) .
\end{aligned}
$$

By neglecting the second order dispersion parameter $(\varrho)=0.5 \Gamma_{R}^{2} \Phi^{\prime \prime}(\omega)$ it follows that

$$
\begin{aligned}
V_{R}(\omega) & =e^{-\frac{1}{4} \Gamma_{R}^{2} \Phi^{\prime 2}} \\
\quad \text { and } & \\
\Phi_{R}(\omega) & =\Phi(\omega) .
\end{aligned}
$$


From

$$
\begin{aligned}
\Delta \lambda_{R} & =\Delta \lambda_{R}^{\prime} \sqrt{2} \\
\text { and } & \\
\Delta \lambda_{R}^{\prime} & =\lambda^{2} \frac{\Gamma_{R}}{2 \pi c} .
\end{aligned}
$$

it follows that

$$
\Gamma_{R}^{2}=2(\pi c)^{2}\left(\frac{\Delta \lambda_{R}}{\lambda^{2}}\right)^{2} .
$$

The final expression for the spectral intensity of a Mach Zehnder is obtained by combining equations B.5, B.6 and B.8:

$$
\begin{aligned}
& S_{M Z}(\omega)=I_{(i n)}\left(1+V_{A} V_{P}(\omega)\right) e^{-\frac{1}{4} \Gamma_{R}^{2} \Phi^{\prime 2}} \cos \left(\frac{\omega}{c} \Delta_{M Z}(\omega)\right) \\
& S_{M Z}(\lambda)=I_{(i n)}\left(1+V_{A} V_{P}(\lambda)\right) e^{-\frac{(\pi c)^{2}}{2}\left(\frac{\Delta \lambda_{R}}{\lambda^{2}}\right)^{2} \Phi^{\prime 2}} \cos \left(\frac{2 \pi}{\lambda} \Delta_{M Z}(\lambda)\right) \\
& S_{M Z}(\lambda)=I_{(i n)}\left(1+V_{A} V_{P}(\lambda)\right) e^{-\frac{\pi^{2}}{2}\left(\frac{\Delta_{M Z}^{g}(\lambda) \Delta \lambda_{R}}{\lambda^{2}}\right)^{2}} \cos \left(\frac{2 \pi}{\lambda} \Delta_{M Z}(\lambda)\right) .
\end{aligned}
$$

\section{B.2 Implementation}

Equation B.10 was implemented in a MATLAB script (R2017b, the MathWorks, Natick, MA, USA) to determine the spectral transmission of a spectrometer with a resolution of $0.6 \mathrm{~nm}$ and a data interval of $10 \mathrm{pm}$ for 8 optical path differences $(0.30 \mu \mathrm{m}, 0.31 \mu \mathrm{m}$, $0.32 \mu \mathrm{m}, 0.33 \mu \mathrm{m}, 0.34 \mu \mathrm{m}, 59.5 \mu \mathrm{m}, 150 \mu \mathrm{m}$ and $300 \mu \mathrm{m})$. The calculations were performed using the effective refractive index ( $\lambda$ dependent $)$ of the second designed crosssection of the buried waveguides. A recorded spectrum of the SLD was normalized and used for $\mathrm{I}_{(\mathrm{in})}$ and the visibility terms were set to one. The expected transmission for the different geometrical path differences are presented in figure B.1.
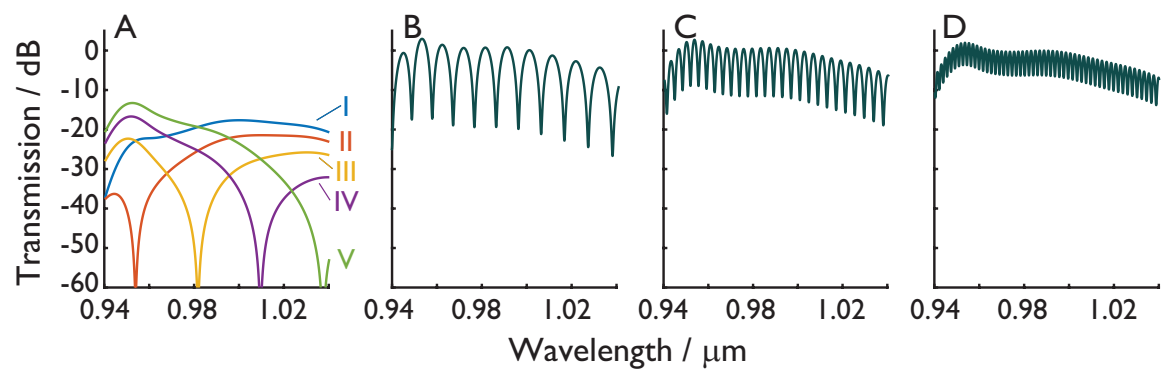

Figure B.1: The spectral transmission determined for a geometric path different of A) $0.30 \mu \mathrm{m}$ to $0.34 \mu \mathrm{m}$, B) $59.5 \mu \mathrm{m}, \mathrm{C} 150 \mu \mathrm{m}$, and D) $300 \mu \mathrm{m}$. An extra difference in optical path can be controlled by applying current to the heaters. Subfigure A shows the transmission response for a small change in path length: $\mathrm{I}=0.30 \mu \mathrm{m}, \mathrm{II}=0.31 \mu \mathrm{m}, \mathrm{III}=0.32 \mu \mathrm{m}, \mathrm{IV}=0.33 \mu \mathrm{m}$, and $\mathrm{V}=0.34 \mu \mathrm{m}$. 
The limited coherence length of the MZI causes the null depth to degrade for larger path length differences. Some additional calculation were performed and presented in figure B. 2 to show the influence of the spectral resolution (of the recording device) and the path difference between the two arms. A data interval of $4 \mathrm{pm}$ was used. The null depth was determined using the same data processing algorithm used for the experimental data (described in 5.8.2). A deeper null depth can be expected if the fringes can not be resolved with a recording device.
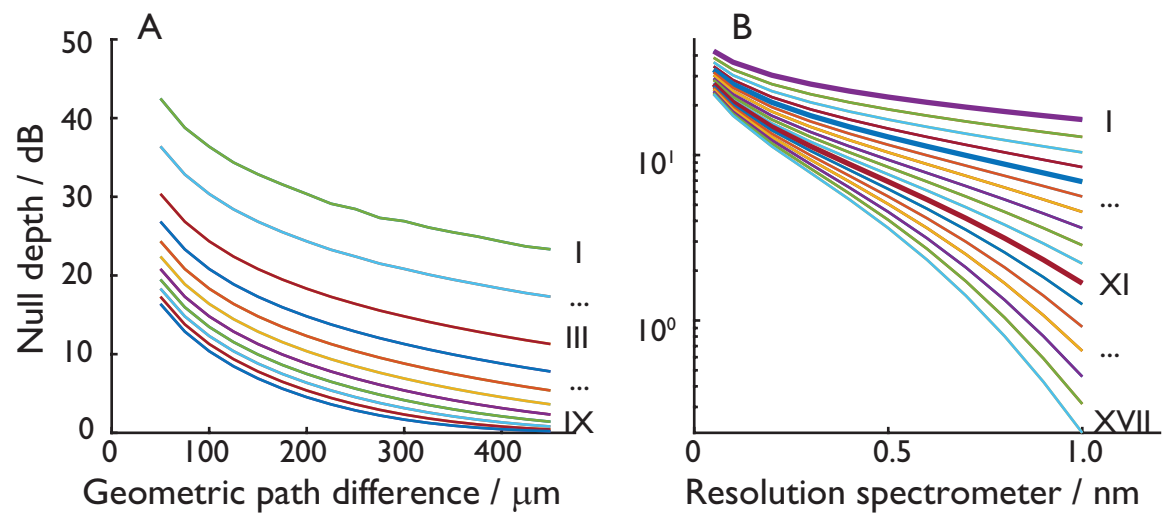

Figure B.2: The effect of optical path differences and resolution of the recording device on the null depth. A) Line plots marked with roman letters I-IX for the resolution of $0.05 \mathrm{~nm}, 0.1 \mathrm{~nm}, 0.2 \mathrm{~nm}$, $0.3 \mathrm{~nm}, 0.4 \mathrm{~nm}, 0.5 \mathrm{~nm}, 0.6 \mathrm{~nm}, 0.7 \mathrm{~nm}, 0.8 \mathrm{~nm}, 0.9 \mathrm{~nm}$ and $1.0 \mathrm{~nm}$. B) Line plots marked with roman letter I-XVII for the optical path difference of $50 \mu \mathrm{m}$ to $450 \mu \mathrm{m}$ in steps of $25 \mu \mathrm{m}$.

The spectral fringe spacing almost increases linear with wavelength for a fixed geometric path difference, showing that the dispersion between the two wavelengths is limited. A linear regression fit is made to determine the fringe spacing at $940 \mathrm{~nm}$ and $1040 \mathrm{~nm}$ for different geometric path differences in our chip design. The result from the Matlab calculation is presented in figure B.3. The fringe spacing $L_{\mathrm{s}}$ determined for the geometric path difference $\left(L_{\text {geo }}\right)$ can be describe with $L_{s}=516.7 / L_{\text {geo }}$ and $L_{s}=647.3 / L_{\text {geo }}$ for the wavelength $940 \mathrm{~nm}$, and $1040 \mathrm{~nm}$, respectively.

In chapter 2 the Gaussian curve fitting of the spectral induced changes on the absorbance spectrum of water is introduced. In this work three parameters of 4 Gaussian curves, (changes in) amplitude, position and width), are chosen to represent the absorbance spectra of 16 electrolytes of $0.5 \mathrm{M}$. The water spectrum (without ionic content) can similarly be described by 4 Gaussian curves. Therefore, the differential absorbance (fingerprint of the ionic content) can be represented by 8 Gaussian curves. Figure B.4 shows the linear fitting of the three parameters for each Gaussian curve that represent $\mathrm{PbNO}_{3}$.

Thus the absorbance of an electrolyte $\left(A_{\mathrm{e}}\right)$ can be expressed in terms of the absorbance of water $\left(A_{\mathrm{w}}\right)$ and Gaussian parameter as: $A_{\mathrm{e}}=A_{\mathrm{w}}+A_{\mathrm{w}} \rho$, where $\rho$ stands for the 


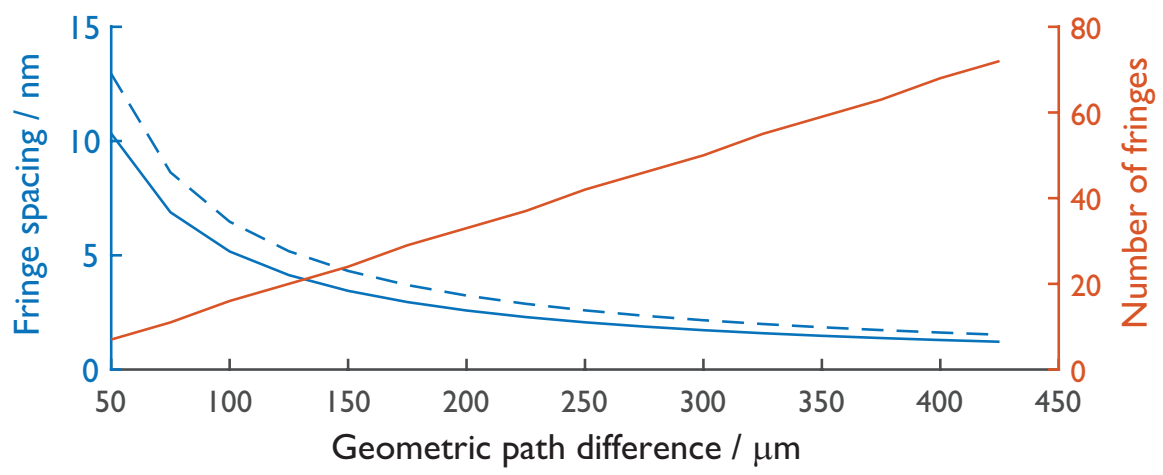

Figure B.3: The number of fringes and their spacing is determined for the chosen $\mathrm{Si}_{3} \mathrm{~N}_{4}$ core with the different geometrical path differences.

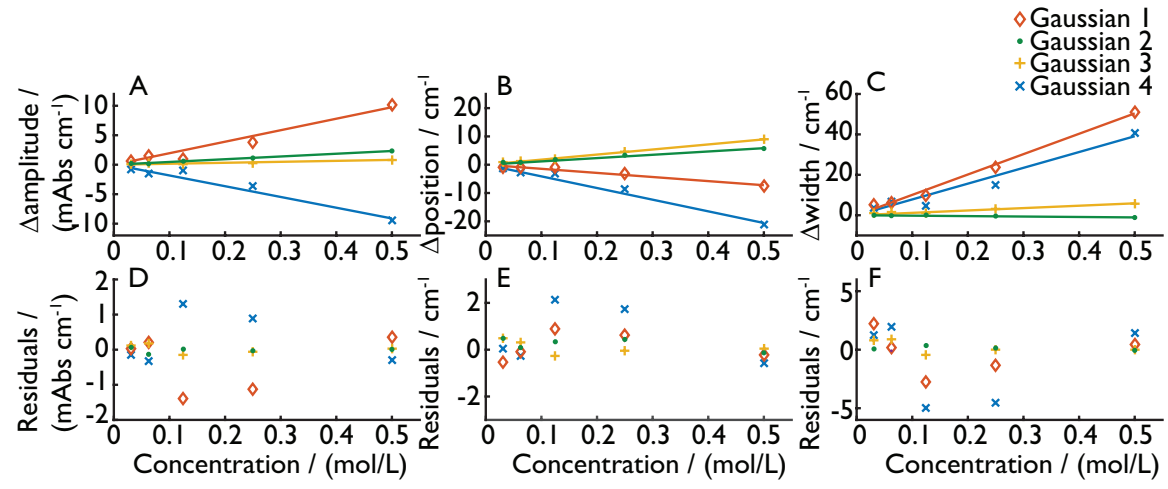

Figure B.4: Linear regression fits of the Gaussian curves representing $\mathrm{PbNO}_{3}$ (as presented in chapter 2). A) The four Gaussian amplitudes as a function of electrolyte concentration, residuals of the fits are shown in subfigure D. Likewise the position, and width of the four Gaussian curves are plotted in subfigures $\mathrm{B}$ and $\mathrm{C}$ and the residuals of the fits are shown in subfigures $\mathrm{E}$ and $\mathrm{F}$, respectively.

influence of the electrolyte. It is expressed as

$$
\rho=0.04 \sum_{i=1}^{8}\left(a(i) e^{-\left(\frac{\lambda-\mu(i)}{\sqrt{2} \sigma(i)}\right)^{2}},\right.
$$

where $a, \mu, \sigma$ denote amplitude, position, and width of the Gaussian curves, respectively. The visibility term due to power imbalance $\left(V_{\mathrm{p}}\right)$ is used to include the absorbance losses of light due to water and the electrolyte in the arms of a MZI:

$$
V_{P}=2 \frac{\sqrt{0.5 I_{(i n)} 10^{A_{w}} 0.5 I_{(i n)} 10^{A_{w}(1+\rho)}}}{0.5 I_{(i n)} 10^{A_{w}}+0.5 I_{(i n)} 10^{A_{w}(1+\rho)}} .
$$

By implementing equation B.12 in equation B.10 the expected visibility and null depth 
degradation due to ionic content can be determined as shown in figure B.5.
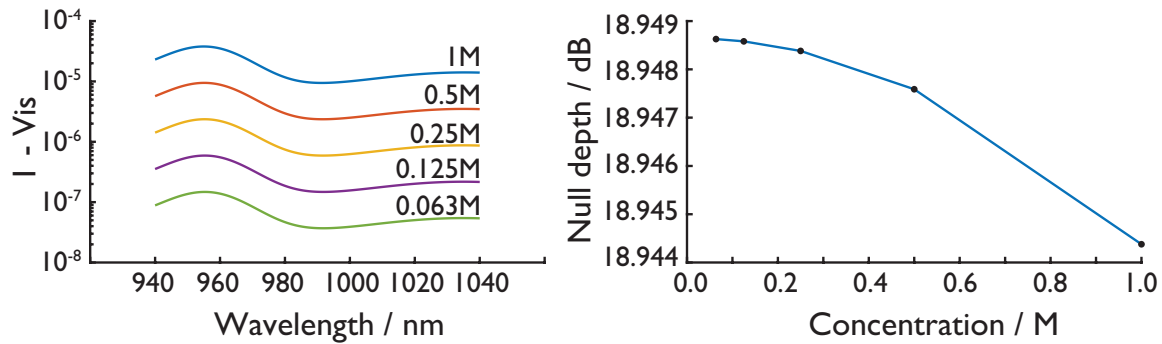

Figure B.5: Calculated spectral visibility and null depth against concentration of $\mathrm{Na}_{2} \mathrm{SO}_{4}$.
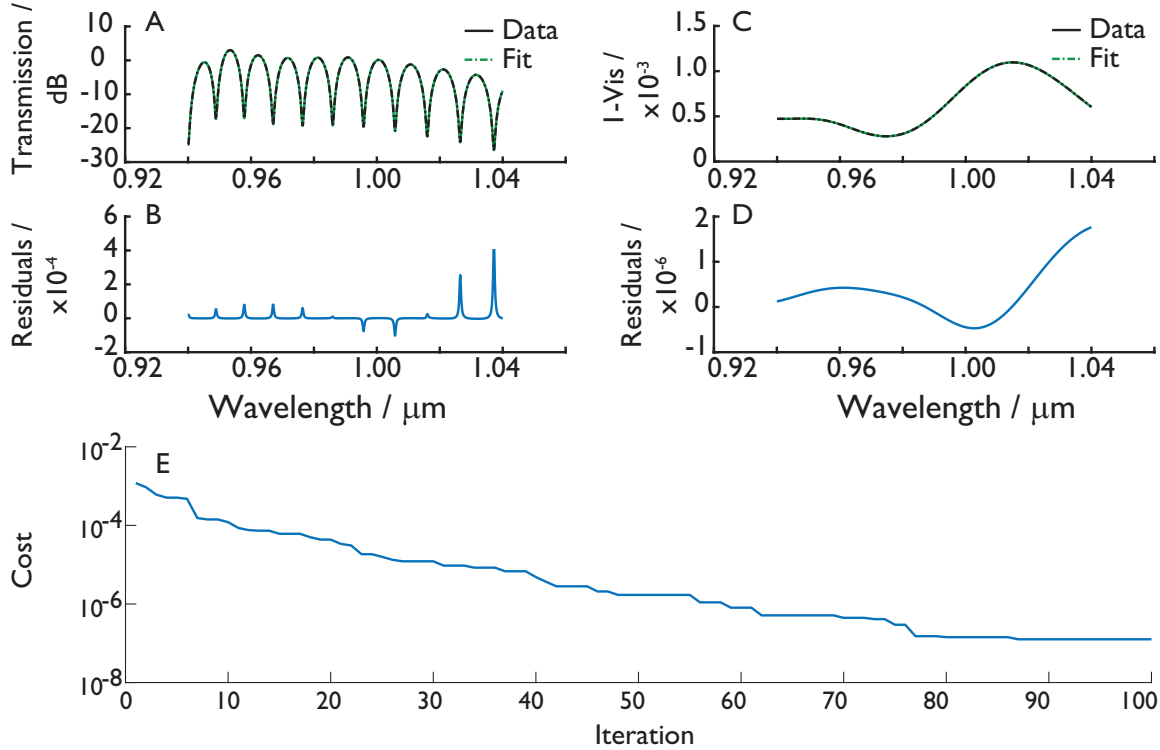

Figure B.6: The performance of fitting a mixture of four electrolytes. A) The determined spectral transmission and its fit, B) residual of the transmission data, C) expected visibility data and its fit, D) residuals of the visibility fit, and E) the performance of the fit.

Interferometric data can be fitted to extract the intensity difference between light propagating in the two arms of an MZI. We used Matlab to create an algorithm based on particle swarm optimization (PSO) algorithm which initiates, similar as a genetic algorithm, a population of random solutions in a search space. The first differs from the latter by an assigned random velocity given to each potential solution (particles) whereby the particles are then 'flown' through the hyperspace. It was inspired by the social behavioral of flock of birds and school of fishs. ${ }^{2}$ The constriction coefficients proposed

${ }^{2}$ Eberhart, R., \& Kennedy, J. (1995, October). A new optimizer using particle swarm theory. In Micro 
by Clerc and Kennedy are included in the script. ${ }^{3}$ First some data is simulated to test the fitting algorithm. The differential absorbance of a mixture of 4 electrolytes, $0.03 \mathrm{M}$ $\mathrm{Na}_{2} \mathrm{SO}_{4}, 0.01 \mathrm{M} \mathrm{NaF}, 0.03 \mathrm{M} \mathrm{NaNO}_{2}$, and $0.02 \mathrm{M} \mathrm{NaNO}_{3}$, is included in the visibility term by assuming the signal of the mixture to be a summation of the individual electrolyte spectral signatures. For the fitting a population size of $1 \times 10^{5}$ and a $1 \times 10^{2}$ iterations were chosen. The script searches for a global minimum for the four concentration in a range of $0 \mathrm{M}$ to $1 \mathrm{M}$ of the electrolytes. Figure B.6 shows a successful fit of the data. The concentration of the electrolytes are found with a $>98.5 \%$ accuracy. A higher population size is required to include more electrolyte in the mixture .

Machine and Human Science, 1995. MHS'95., Proceedings of the Sixth International Symposium on (pp. 39-43). IEEE.

${ }^{3}$ Clerc, M., \& Kennedy, J. (2002). The particle swarm-explosion, stability, and convergence in a multidimensional complex space. IEEE transactions on Evolutionary Computation, 6(1), 58-73. 


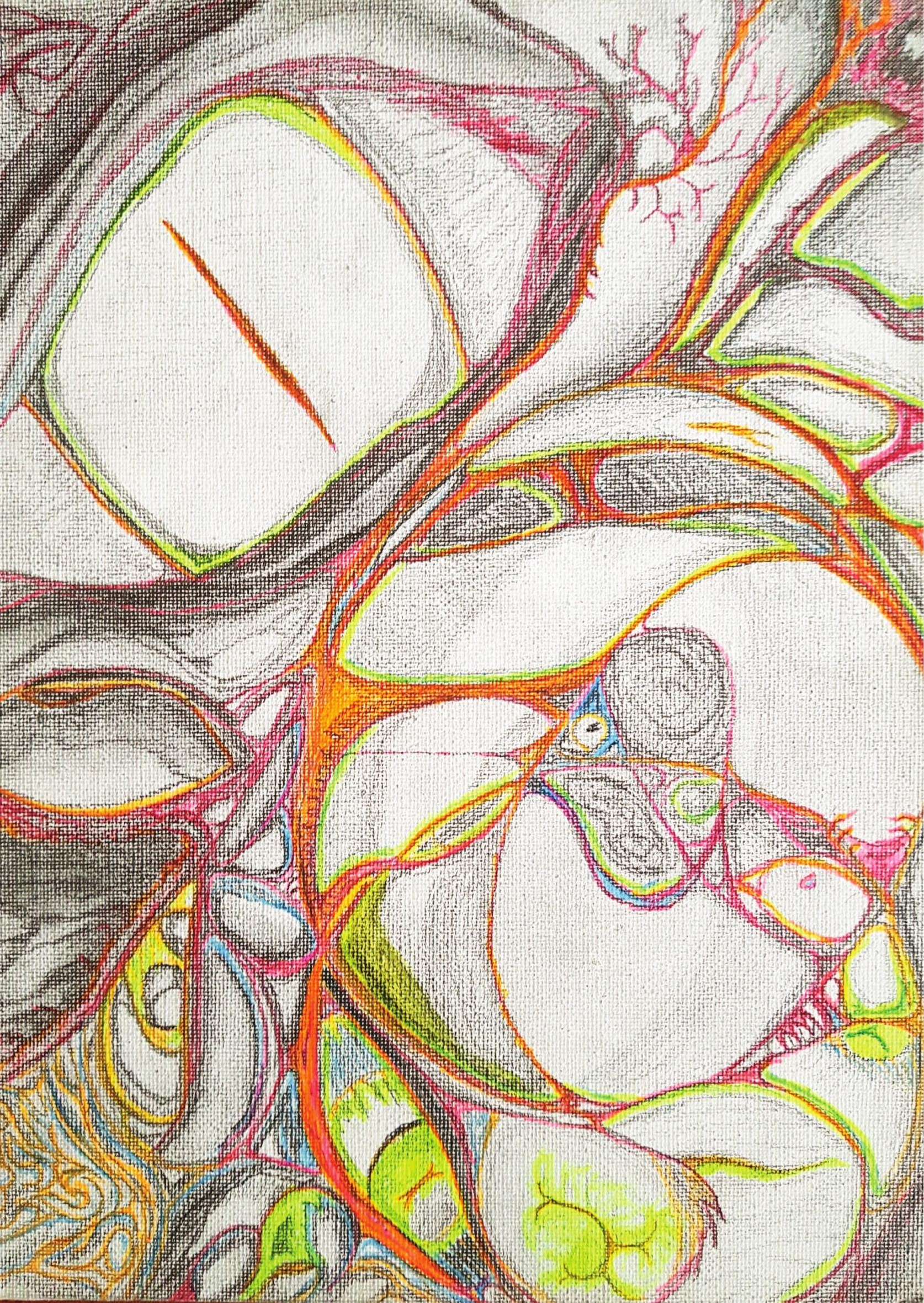




\section{APPENDIX}

\section{Microscopic images - first batch optofluidic chips}

This appendix contains the microscopic images taken of the first batch of chips to give an overview of these chips with and without any visual defects. Furthermore images of the light scattering in the chips are presented. Figure C.1 shows the labeling of the optofluidic chips and is used throughout appendix $\mathrm{C}$. The fabrication of optofluidic chips is a complicated, multistep complex process. For the first run an average chip yield of $70 \%$ was expected. The orange-brown crosses (overlayed on some microscopic images) indicate an issue with the top cladding at the coupling interfaces. These interfaces are marked by orange-brown ellipses. The blue crosses on the images indicate a problem with in the sensing window(s), such as under-etch, broken top cladding or glue deposition.
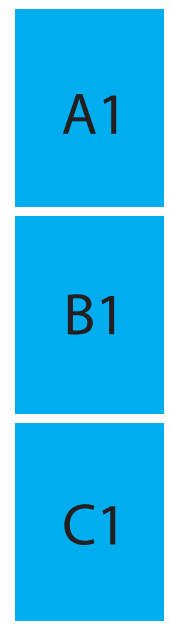
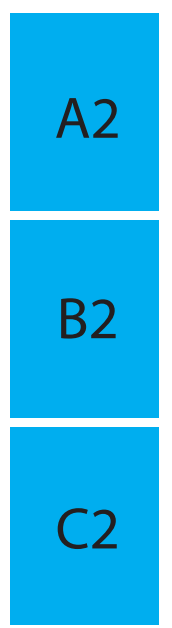
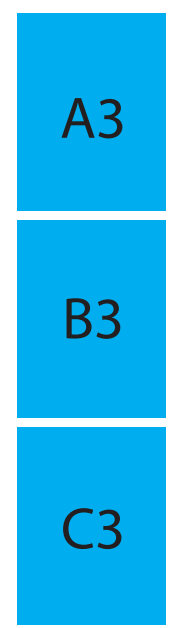
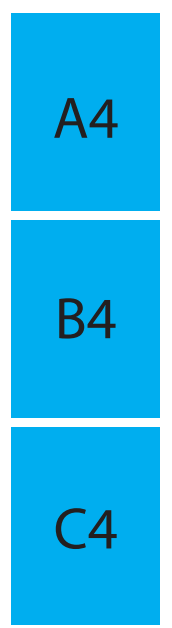
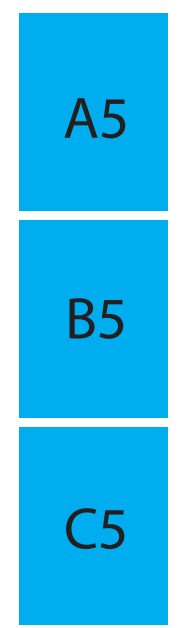
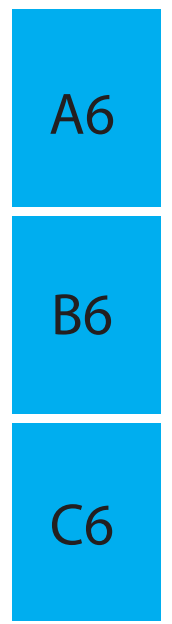

Figure C.1: Logic naming scheme that is used for the diced chips. 

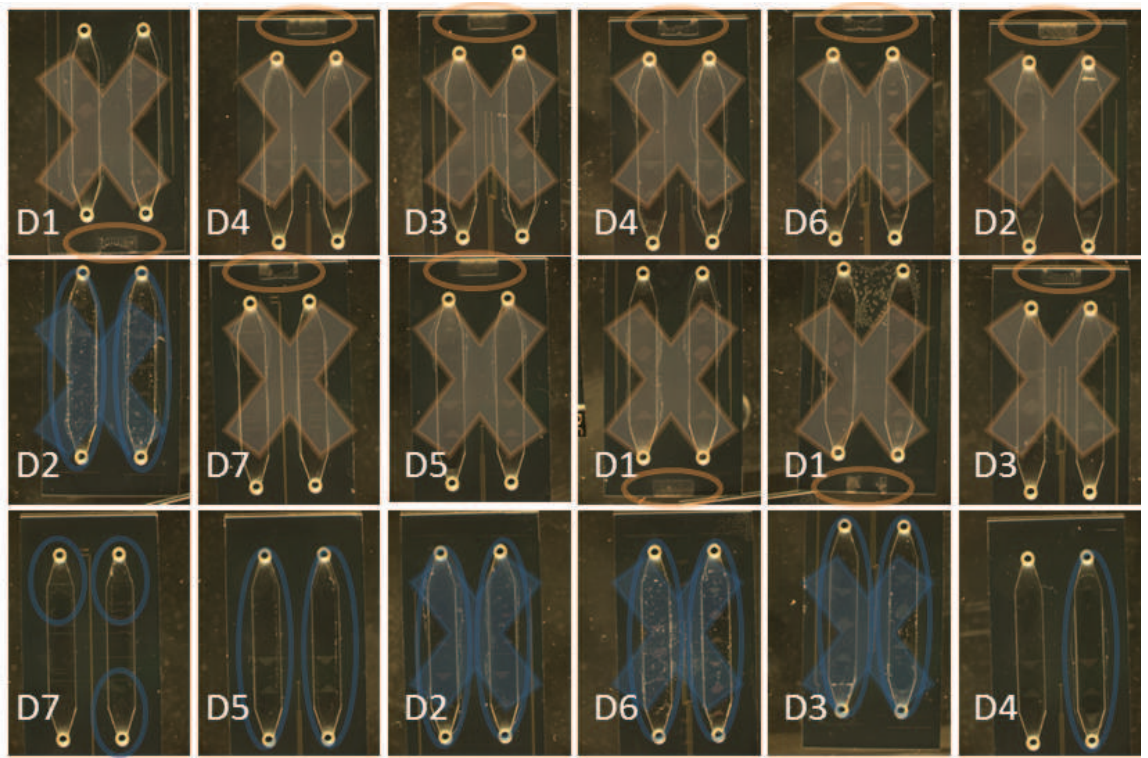

Figure C.2: Images of optofluidic chips diced from wafer 3058. The wafer had a low chip yield due to the topcladding quality of the chips. The optofluidic chips $\mathrm{C} 1, \mathrm{C} 2$ and $\mathrm{C} 6$ have the least amount of observed defects.

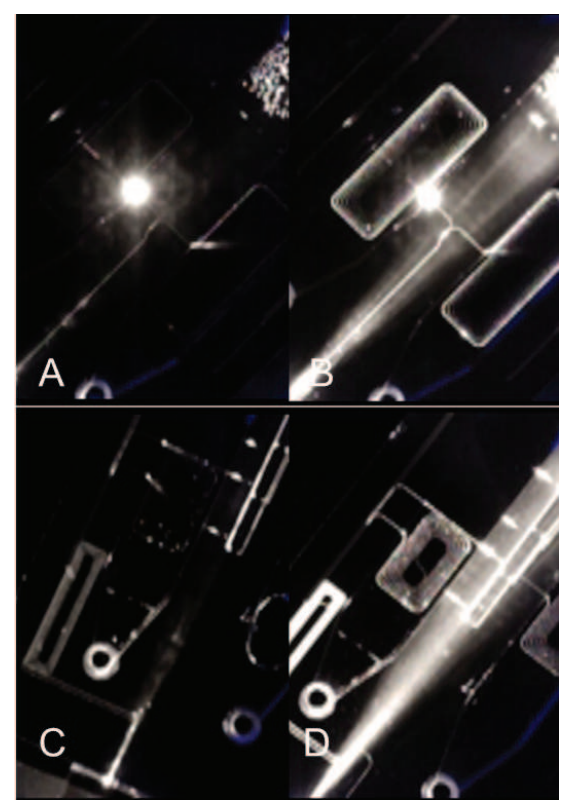

Figure C.3: Images taken after light is coupled to the chips of wafer 3058. A) TM propagation through chip B5 (design 1). B) TE propagation through chip B5. C) TM propagation through chip C5 (design 3). C) TE propagation through chip C5. 


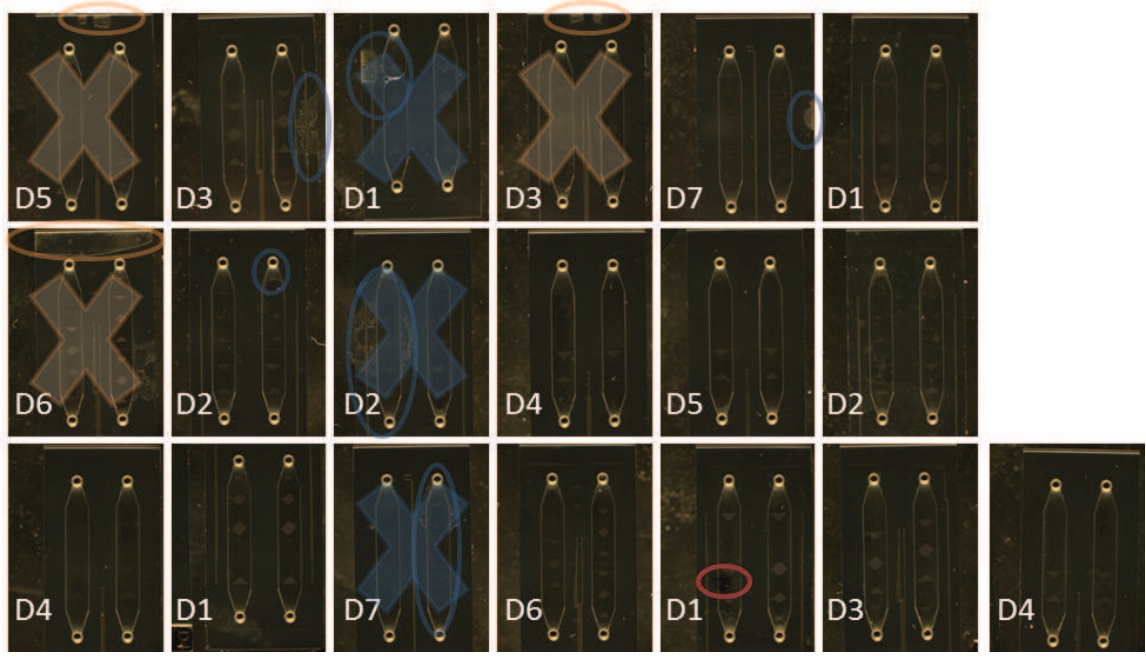

Figure C.4: Images of optofluidic chips diced from wafer 3089. The wafer appears to have a decent yield of $\sim 66 \%$.

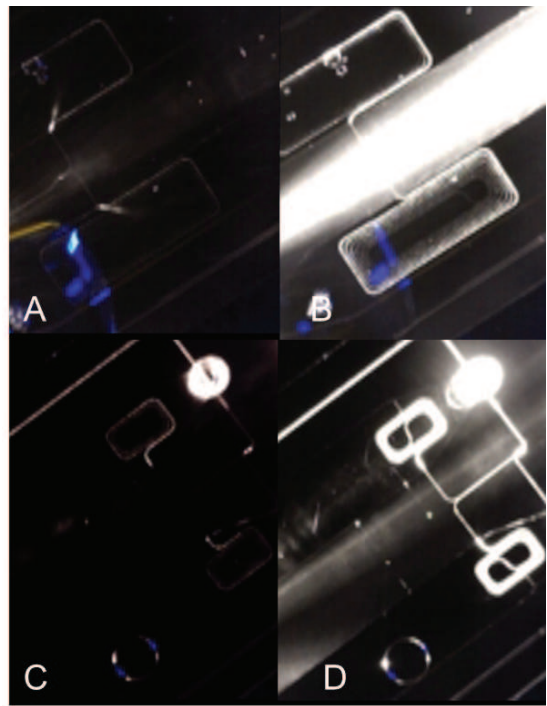

Figure C.5: Image of the light scattering, recorded from two chips of wafer 3089. A) TE SLD propagation on chip A3. B) TM SLD propagation on chip A3. C) TE SLD propagation on chip C2. D) TM SLD propagation on chip C2. 

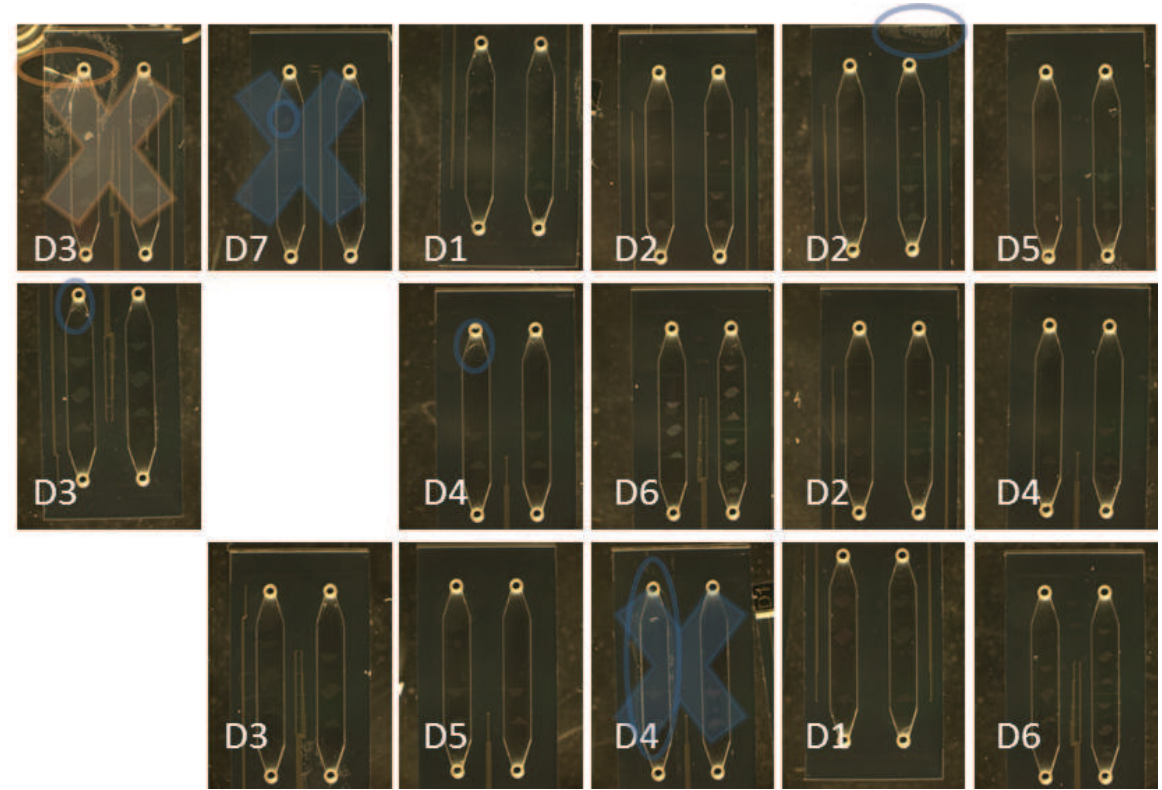

Figure C.6: Microscopic images of optofluidic chips diced from wafer 3091. The wafer appear to have a decent yield of about $66 \%$.

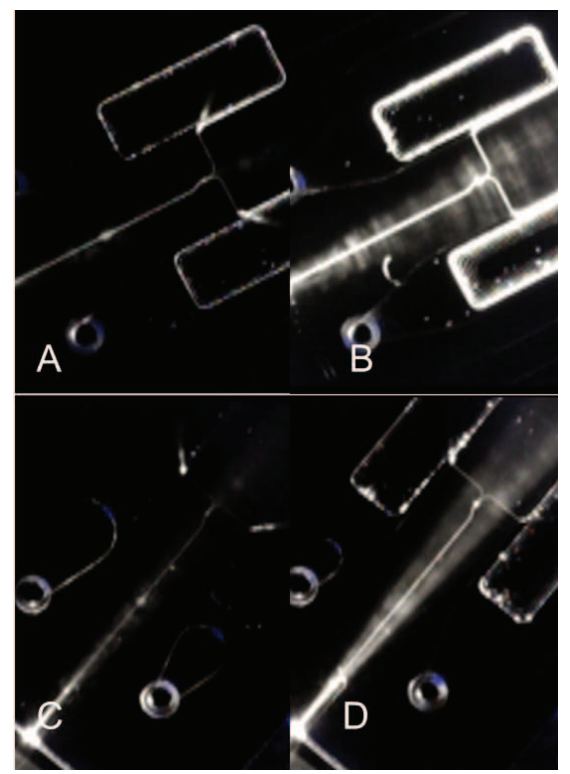

Figure C.7: Image of the light scattering, recorded from two chips of wafer 3091. A) TE SLD propagation on chip C5 (design 1). B) B) TM SLD propagation on chip A3 (design 1). C) TE SLD propagation on chip C2. D) TM SLD propagation on chip C2. 

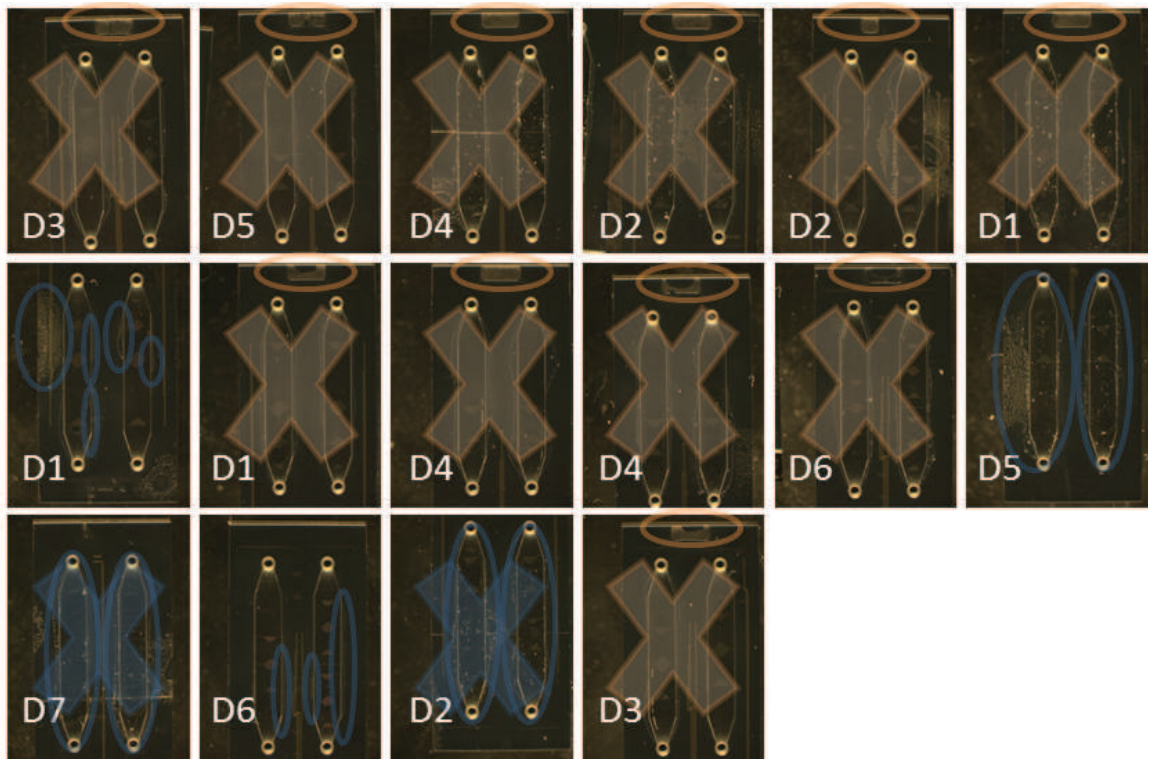

Figure C.8: Images of optofluidic chips diced from wafer 3112 . The quality of the topcladding was low in wafer.

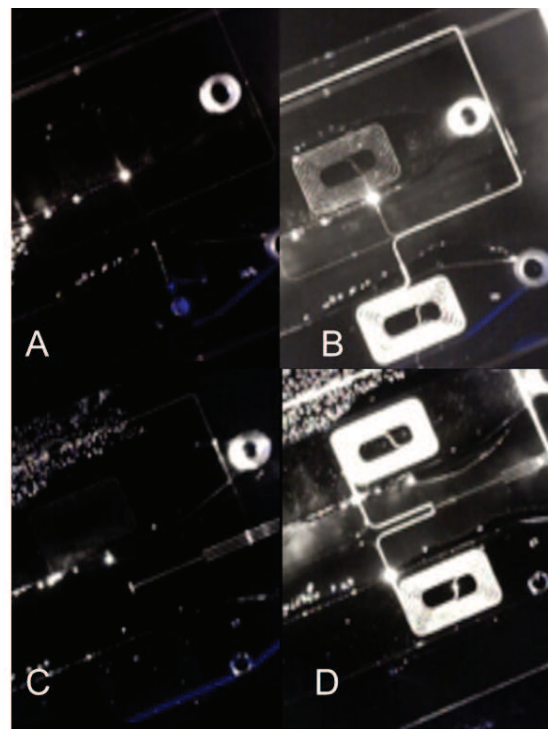

Figure C.9: Image of the light scattering, recorded from two chips of wafer 3112. A) TE SLD propagation on chip C3 (design 2)). B) TM SLD propagation on chip C3 (design 2). C) TE SLD propagation on chip B6 (design 5). D) TM SLD propagation on chip B6 (design 5). 

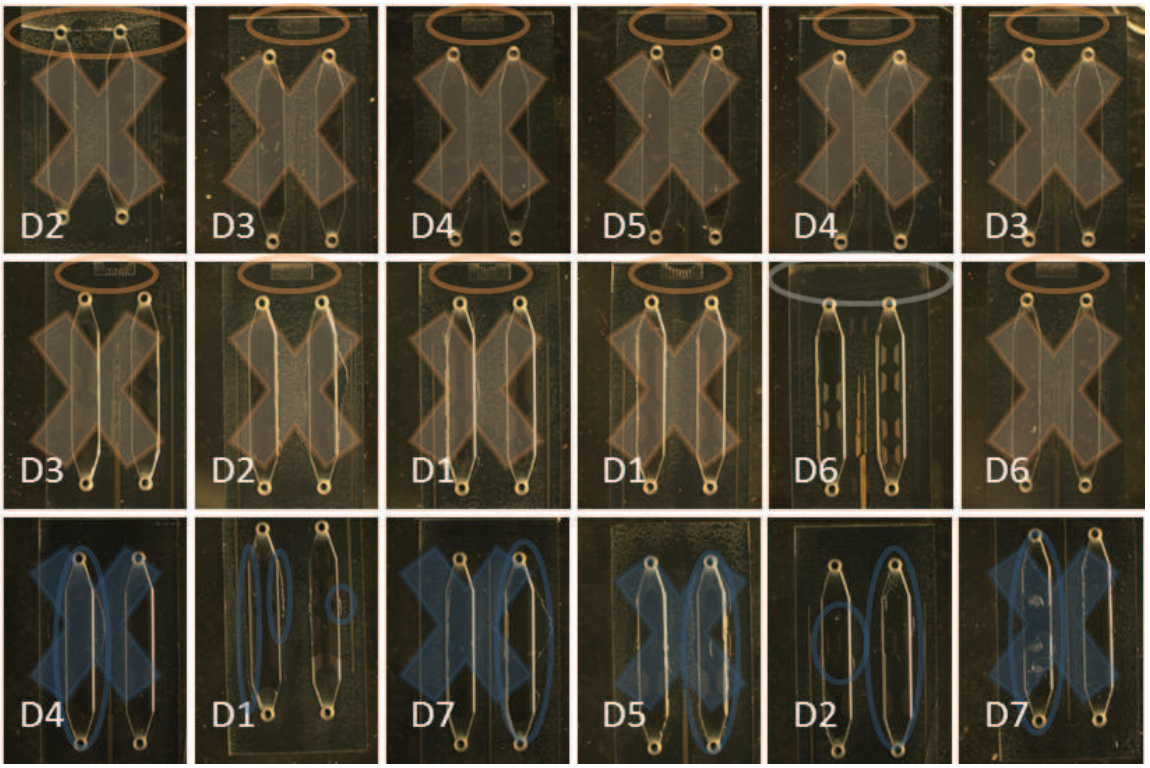

Figure C.10: Images of optofluidic chips diced from wafer 3133 . The quality of the topcladding in this wafer caused a low chip yield.

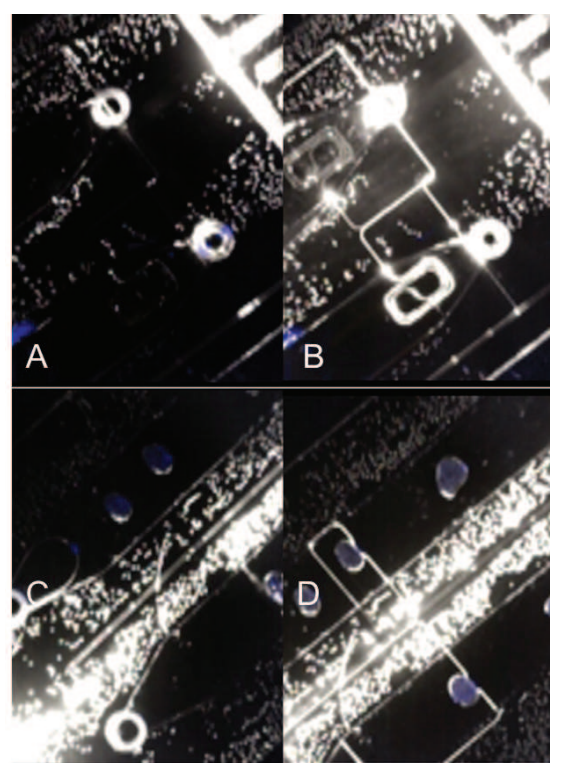

Figure C.11: Image of the light scattering, recorded from two chips of wafer 3112. A) TE SLD propagation on chip C2 (design 1). B) TM SLD propagation on chip C2 (design 1). C) TE SLD propagation on chip C7 (design 7). D) TM SLD propagation on chip B7 (design 7). 




\section{APPENDIX \\ Mask design - second batch optofluidic chips}

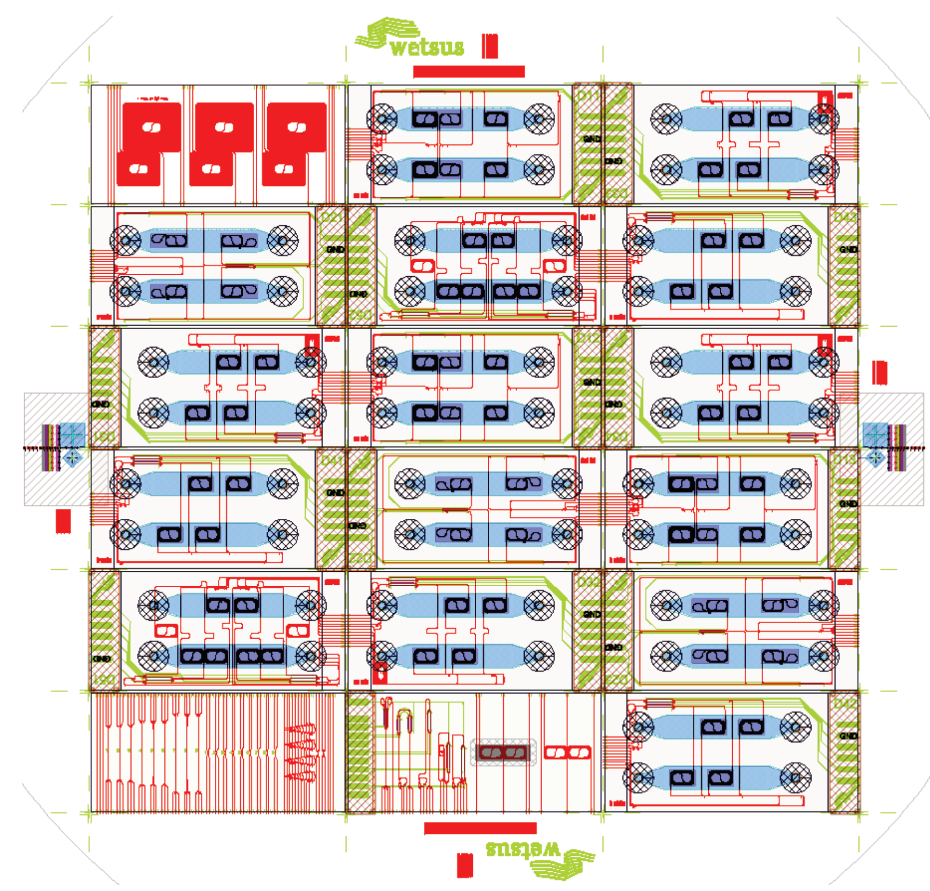

Figure D.1: Maskdesign used for fabrication of the second batch of optofluidic chips. Green indicates positions for gold deposition. Blue marks the fluidic windows, and darker blue marks etching areas to enable sensing. The waveguides are indicated with red color. 


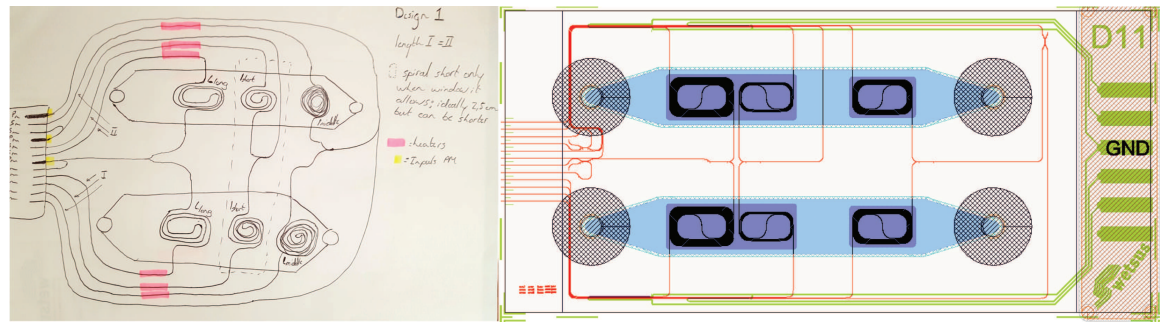

Figure D.2: The optical layout to record the absorbance in two fluidic channels. Three design are put on maskdesign $D_{11-13}$ to allow for electronic subtraction with three sensing lengths. The position of the maskdesigns on the wafer is the only difference between maskdesign $\mathrm{D}_{11}, \mathrm{D}_{12}$, and $\mathrm{D}_{13}$. Light coupled to input 1,4 , and 7 can be partly monitored at output 12,3 , and, 8, respectively. The bisected light propagates through the $5 \mathrm{~cm}, 2.5 \mathrm{~cm}$, and $7.5 \mathrm{~cm}$ spirals and can be recorded at output 2 and 11,5 and 10,6 and 9 , respectively.

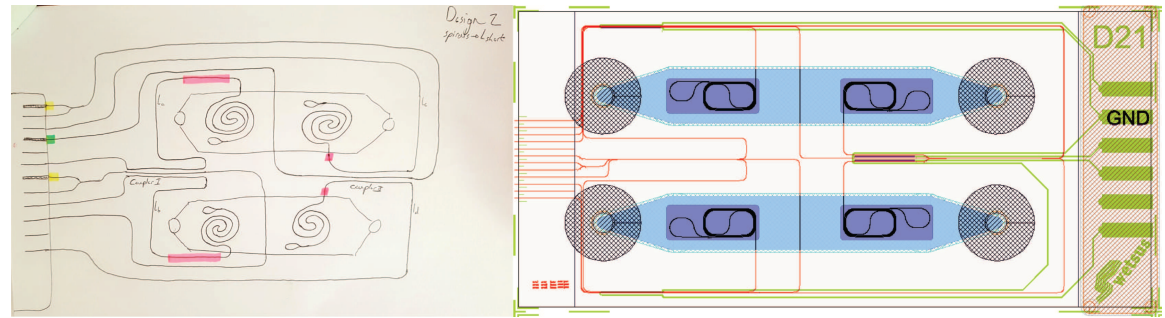

Figure D.3: The optical layout of two Michelson intefermeters are realized on maskdesign $\mathrm{D}_{21-23}$. Geometric path length differences of $59.5 \mu \mathrm{m}$ and $150 \mu \mathrm{m}$ between the two arms were designed to realize 10 and 26 fringes in the bandwidth. $\Xi$ parameter $1 \& 2,3 \& 4$, and $5 \& 6$ were used in the Micheson inteferometers in Maskdesign $\mathrm{D}_{21}, \mathrm{D}_{22}$, and $\mathrm{D}_{23}$, respectively. Part of the light coupled to input 1 is split by the $\Xi$ coupler. Light can be monitored for reference purposes at port 4 . The Michelson interference output can be recorded at port 2. The other Michelson interferometer can be access by coupling light to input 7 . The $\Xi$ coupler divides the light to the two sensing paths. Light can be monitored for reference purposes at port 10. The Michelson interference output can be recorded at port 8 . The performance of the $\Xi$ coupler can be determined by coupling light to input 4 and 10 and recording the intensity at port $6,7,8$ and 9 , and 1,2,3 and 12 , respectively. 


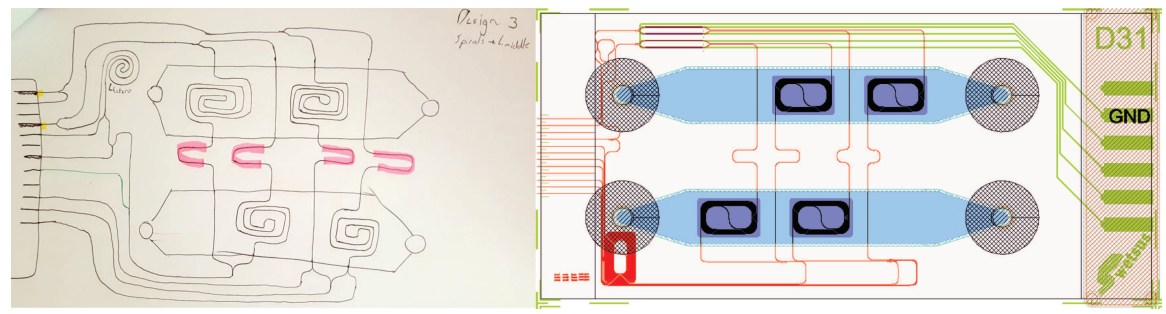

Figure D.4: Maskdesign $\mathrm{D}_{31-34}$ contains two optical layouts. The first is a MZI and the second holds a MZI and a homodyne interferometer. Geometric path length differences of $0.3 \mu \mathrm{m}, 59.5 \mu \mathrm{m}$, $150 \mu \mathrm{m}$ and $300 \mu \mathrm{m}$ between the two arms of the first optical layout were designed to realize 0 , 10, 26 and 52 fringes in the bandwidth in the output of the MZI on D31, D32, D33, and D34, respectively. The MZI of the second optical layout had designed geometric path length differences of $0.3 \mu \mathrm{m}$ and $59.5 \mu \mathrm{m}$ on maskdesign $\mathrm{D}_{31-33}$, and maskdesign $\mathrm{D}_{34}$, respectively. The geometric path length of the local oscillator is $900 \mu \mathrm{m}, 1050 \mu \mathrm{m}, 1150 \mu \mathrm{m}$ and $1050 \mu \mathrm{m}$ smaller than the sensing paths of the MZI which with is it recombinated, resulting in an additional modulation of 35,61 and 68 fringes in the spectra output.
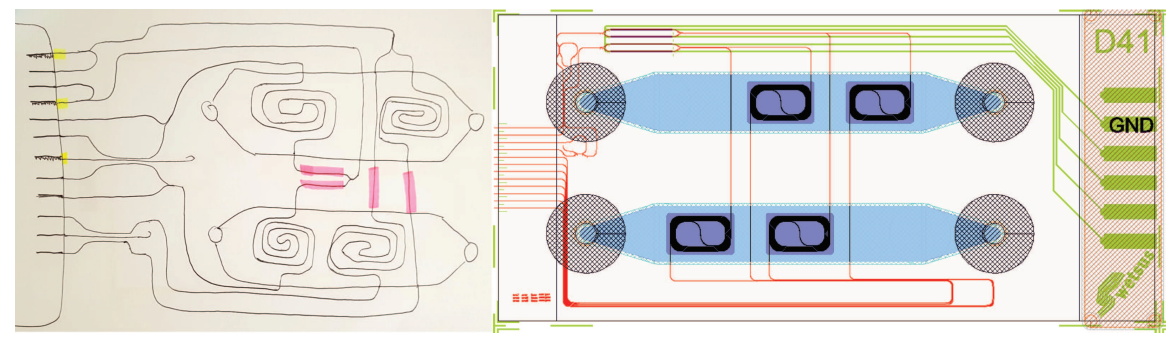

Figure D.5: The optical layout of two MZI are realized on maskdesign $\mathrm{D}_{41-43}$. Geometric path length difference of $0.3 \mu \mathrm{m}$ are designed between the two arms of the interferometers. $\Xi$ parameters $7 \& 2,8 \& 4$, and $9 \& 6$ were used in the MZI in maskdesign $\mathrm{D}_{41}, \mathrm{D}_{42}$, and $\mathrm{D}_{43}$, respectively. Part of the light coupled to input 1 is split by a y-junction to monitor the power fluctuation at port 2 . Another Y-junction is used to bisect the light and after the $5 \mathrm{~cm}$ sensing paths are recombined using a $\Xi$ coupler. The outputs can be recorded at port 10,11 and 12. The other MZI can be access by coupling light to input 4 . A y-junction splits part of the light to monitor the power fluctuation at port 3. Another y-junction is used to bisect the light. After propagating through the sensing paths the light is bisected to monitor the power after the sensing paths at port 5 and 9 . The light is recombined with a $\Xi$ coupler and its output can be recorded at port 6,7 and 8 . 

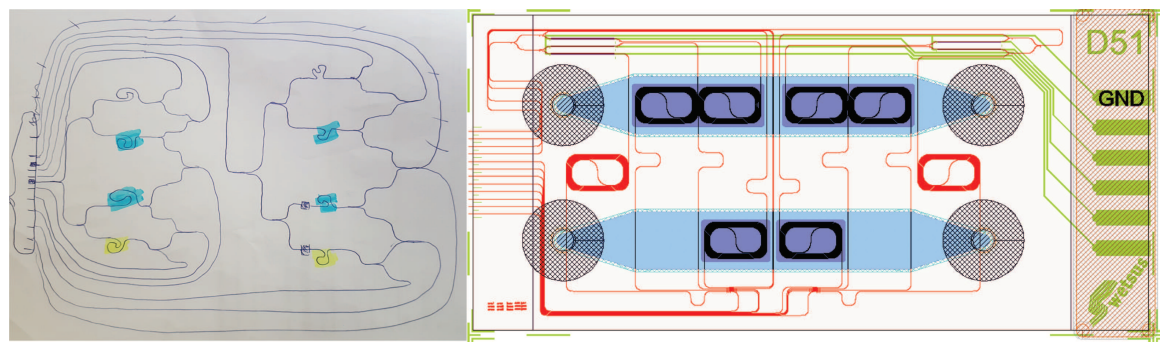

Figure D.6: The optical layout of the online sensor with internal calibrator is realized twice on maskdesign $\mathrm{D}_{51-52}$. The input ports are 1 and 4 . Light propagating through the buried spiral, and sample spiral can be recorded at 5 and 12, and 2 and 8, respectively. The inference of recombination of light propagating through the two sample spirals, and the sample spiral and reference spiral can be recorded at 7 and 10,3 and 9 . The inference of light propagating through the sample spiral and the buried spiral can be recorded at 6 and 11 .

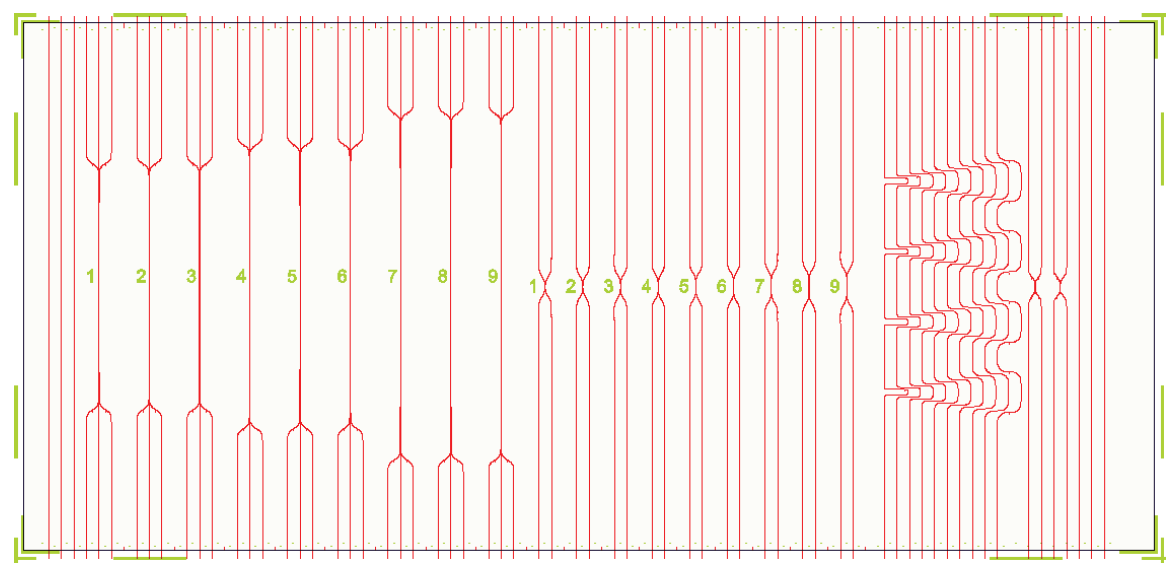

Figure D.7: The optical layout of the teststructures are realized on maskdesign T1. The teststructures contain the $9 \Xi$ coupler with the 9 parameters, 9 bidirectional coupler, 10 different bending radii, another 2 bidirectional coupler and some straight waveguides. 


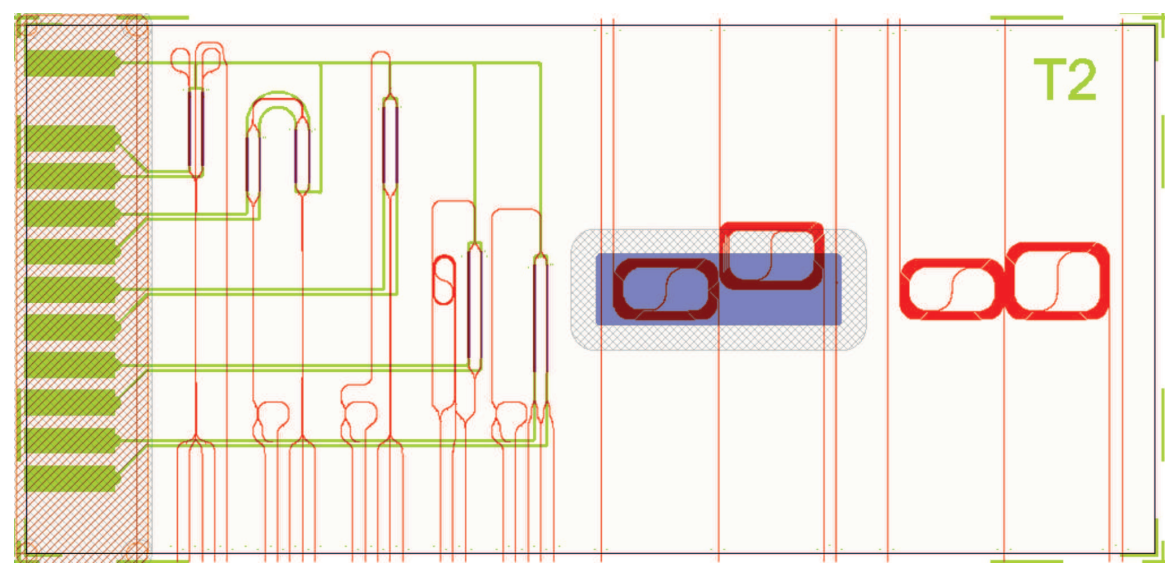

Figure D.8: The optical layout of additional teststructures are realized on maskdesign T2: a Michelson interferometer, two MZI (with $\Xi$ coupler for recombination of the bisected light), a homodyne interferometer, another MZI with an inverted Y-junction for recombinaton, two sensing spirals to determine the interface losses, and finally, two buried spirals to determine propagation losses.

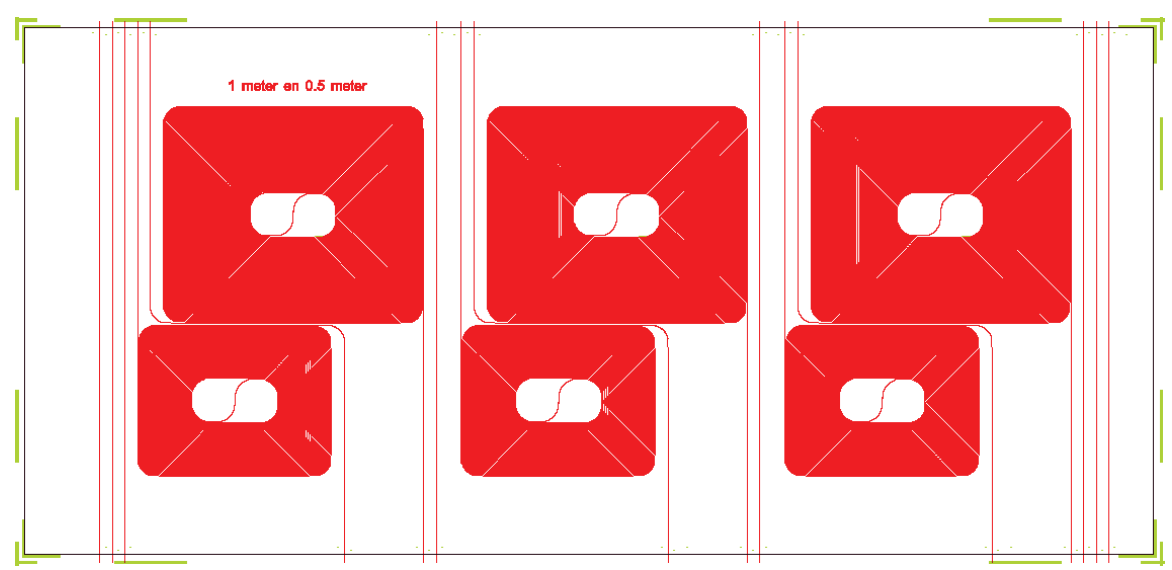

Figure D.9: The optical layout of additional teststructures for determination of propagation losses are realized on maskdesign T3: two long spirals $0.5 \mathrm{~m}$ and $1 \mathrm{~m}$ are positioned on the mask design. 



\section{Algemene Nederlandse samenvatting}

Het doel van dit werk was het ontwikkelen van een optofluidisch $^{1}$ absorptieplatform $^{2}$ om anorganische ionen te kunnen detecteren ${ }^{3}$ in water. In Hoofdstuk 2 wordt de meetmethode geïntroduceerd. Met een spectrometer (apparaat om de absorptie per golflengte vast te leggen) zijn de absorptie verschillen van 16 verschillende elektrolyten ten opzichte van gedemineraliseerd water (water zonder ionen) vastgelegd. Deze bleken uniek te zijn voor de verschillende elektrolyten en het signaal is lineair (2x de hoeveelheid ionen betekent $2 \mathrm{x}$ zo veel absorptie) afhankelijk van de concentratie (aantal ionen in een bepaalde hoeveelheid water). Dit maakt de methode geschikt voor een sensor.

Hoofdstuk 3 borduurt verder met temperatuur als belangrijke parameter. Er is gekeken of de absorptie van een elektrolyt verandert als in het water ook een ander zout wordt opgelost. Daarbij is gebleken dat de zouten invloed hebben op elkaar, maar dat bij hogere temperatuur de aanwezigheid van het ene opgeloste zout minder invloed heeft op het signaal dat veroorzaakt wordt door het andere opgeloste zout.

Geïntegreerde optofluidische sensoren zijn ontworpen uitgaande van een aantal randvoorwaarden en eisen aan de sensor. De eerste set gefabriceerde sensoren weken sterk af van de verwachte resultaten. Daarop zijn de chips onderzocht met verschillende microscopen. Dit is samengevat in hoofdstuk 4. Dit onderzoek bracht fabricage-fouten aan het licht en leidde tot verbeteringen in het ontwerp.

In hoofdstuk 5 wordt dieper in gegaan op de verbeterde ontwerpafwegingen en zijn de eerste resultaten weergegeven van de nieuwe sensoren. Hoewel verder onderzoek noodzakelijk is, kan gezegd worden dat een eerste stap gezet is om ionen in water te kunnen detecteren met integreerde optofluidische sensoren gebaseerd op absorptiespectroscopie. In hoofdstuk 6 worden de algemene conclusie en het toekomstperspectief gepresenteerd.

\footnotetext{
${ }^{1}$ Een optofluidische sensor is een sensor die optische en fluidische componenten combineert om iets te meten en daarbij functionaliteit heeft, die afzonderlijk niet mogelijk is. De sensor die in dit werk is ontwikkeld heeft optische en fluidische componenten op microschaal $(1 \mu \mathrm{m}=0.0001 \mathrm{~cm})$. De belangrijkste redenen om een optofluidische sensor te ontwikkelen, is de wens om 'realtime' op afstand informatie te krijgen over de ionenconcentratie in water (voor zowel de industrie als drinkwaterbedrijven), en de mogelijkheid deze te gebruiken op lastig bereikbare plaatsen, zoals in buizen.

${ }^{2}$ De afname van licht door de lengte, het type, en de hoeveelheid materiaal wordt absorptie genoemd. Zo heeft sterke koffie meer lichtabsorptie dan slappe koffie.

${ }^{3}$ Keukenzout (dat uit natrium- en chloor ionen bestaat) kan worden opgelost in water, zonder dat er sprake lijkt te zijn van een absorptie-verschil van licht ten opzichte van water zonder ionen. Echter, een meterslange buis gevuld met water lijkt blauw, wanneer men erdoorheen kijkt. Water heeft namelijk een hogere absorptie voor rode kleuren dan voor blauwe kleuren. Kleuren (golflengtes) die roder (langer) zijn dan het rood dat waar te nemen is met menselijke ogen, wordt nabij infrarood genoemd. Water absorbeert golflengtes verschillend, dat wil zeggen dat bij sommige golflengtes meer lichtafname plaatsvindt dan bij andere. Een absorptiespectrum laat zien hoeveel licht wordt geabsorbeerd per golflengte. Het oplossen van zout beïnvloedt het absorptiespectrum van water.
} 


\section{Acknowledgements}

66 Little by little, one travels far.

J.R.R. Tolkien,

This journey has ended. The first step towards the adventure started at the University Twente by recording the absorption spectra (i.e., the amount of intensity reduction of different colors) of many different aqueous samples (i.e., water with dissolved salt). An interesting article in astrophysics sparked the beginning of a new direction. The authors used a method called 'nulling' to observe starlight reflected by a neighboring planet. The reflection is orders of magnitudes smaller than the light directly from the star. The absorbance difference due to low $(\mathrm{mM})$ concentrations of ions is also order of magnitudes smaller than the absorbance of light by the water molecules. Thus, we set out to apply the method to our sensing challenge. The road changed from a simple and straight path to a complicated entanglement of potential paths. An interferometric setup was build to test implementation of a similar method. The available lab space and equipment led to a quick preparation of the setup, though the alignment of the optical components took considerably more time. Thank you Dirk Jan for sharing the optical table with me so that I could start playing with the optical components. I moved my setup within the optical sciences (OS) group to establish together with Lourens the MaF (Microscopy and Fluidics) lab only a few months later. Thank you man for the awesome playlist and the fun. Many others from Twente I would like to give thanks. Jeroen for your help to realize the ovens for the photospectrometer and the controls you made for the heaters on the chip. Frans for guiding me to place my first order and later with the SEM recordings. Jorick and Erik for introducing me to the hot sauce, I still regularly use it. Jennifer, Annemarie, Sonia, Qing, Divia, Jorick, Sandra, Carlijn, Raimond, Michiel, Ward, Wooje, Simen, Jinfeng, Pablo, Teus, Bente and many others from the OS family for the cozy atmosphere. I had an awesome time in Twente, playing with alignment sometimes till deep in the night. Simon and Thomas for blasting music. Tom for helping me to find a suitable translation stage. I fear I still have forgotten many names, I will buy you a drink.

The journey took me after $\sim 18$ months to Leeuwarden. The optical lab at Wetsus was recently built by Adam with the assistance of Joeri. I am grateful for the time we spent together and the friendships that were formed. Waiting for me was a 4 by 4 meter facility without yet any optical components suitable for the Near Infra spectral region and an empty 1 by 1.5 meter table. Enthusiastically, I started to set up the third interferometric setup, which was much faster built than the previous two. Due to the 
continuous success of Wetsus, we had to move to a larger building. The optical lab area increased significantly in size and also my optical table: a 3 by 1 meter area was soon occupied by two working setups: one to investigate optical fluidic chips and the second by an automated free space Mach Zehnder interferometer. This work would not have been possible without the support of numerous colleagues and friends in the North. I will have a hard time mentioning everybody who made my Ph.D. time the learning experience it has been, but I will give it a try. First of all, thank you Adam, Elmar and Herman for the numerous conversations and guidance. Herman you had more confidence than me in the process that I went through during the $\mathrm{PhD}$ period. Adam, words elude me to tell you have how much I appreciate your coaching. Elmar, beside helping with the scientific art in the work (making graph is an art), thank you for introducing me to some wicked music. I am looking forward to paint something new together. Cees and Johannes for making Wetsus a reality and supporting the idea that we have much innovate potential in the water technology with optics. Riet and Gerben for making the best soup, Hester for founding such a nice location for the defense. So many people at Wetsus to thank. I would like to thank all the Wetsusians (I am horrible in remembering names), to name a few Wiebe, Willy, Jannie, Tineke, Jaap, Jorrit, Prashant, Pedro, Rik, Jan de G., Henk M., Heleen, Hilde, Albert, Lucy, Claudia, Martijn W., Hector, Fabian, Natascha, Hakan, Casper, Maja, Miriam K., Sonja, Karen, Rotondo (Raimonda), Ricardo. Anke for the many room reservations. Gerrit for handling all the urgent orders. Rienk for always having the right cable within no time. Jan Jurjen I only realized after my the PhD that I could have asked you much more. Maybe my setup would have been less chaotic, nevertheless thank you for the many hours you made stuffs for me and my students. Wim for your trust whenever I have something to purchase. Ingrid for the personal-development courses. Janneke T. for taken safety seriously. Ernst for the discussion we had on chapter 3. Luewton for the proofreading. My students Jurre, Harry, Martin, Juan Sebastián, thank you for the hours you spent on recording interesting data. Chapter 3 would not have been possible without the many hours Phong spend on the project. His sudden death came as a shock.

I am immensely grateful to my paranymphs, Gonçalo and Brahzil for the effort and support. My friends in Vroomshoop, Zeist and Lierop: Sjon \& Kirsten, Bernd, Daan \& Anne, Johan \& April with little Esther for the leisure time. Tim, Slawek, Aga, Joeri for the fun climbing evenings we had. I believe I am slightly more flexible. Hanneke, Maria, Gerrit, Mirella for the interesting philosophical evenings in Groningen. Thank you wonderful people from the 1.18C kingdom; Lena, Paula, Phillip, Gonçalo, future ex-wife Tiki, Victor, Andrew, Wiecher, Thomas, Shuyana, Swarupa. It was fun being in the office and the unforgettable special office BBQs. Patrick you are a very special person, I will not forget the strange conversations. Floris, Marcel, and Lennard for introducing me to Optodesigner. I learned my tricks from the best. Rene and Henk for the given trust. The OFL (Our Future Leaders) course improved both my professional and personal life. For that a special thanks to Geert, Nard, Thomas, Cecile, Sally, Queena, Anouk, Ernst Jan, Ron and Romée. Janneke D. and alle leute von der Fähnlein for the LARP events. The DnD nights with Pau, Olivier, Adam \& Andrew were unforgettable. How can I ever forget a certain person rubbed poop on his face as a camouflage ... 
My family in Heerenveen; Mira, Salih, Alex and Dida, the Raza family in the Philippines, Grandpa Wim, who inspired me as a kid with cool scientific experiments, $\mathrm{Pa}$ Henk, for listening and giving advices, Ma Gertruud, for inspiring me to aim high, to my siblings who are always there for me. Jeannine \& Maarten and kiddos Joah and Yara, Hetty \& Wouter, Hanneke \& Wouter and my smooshy bears, Brahzil, for making me fat, for the deep and never ending conversations, for your love and endless support. Brianne, for your laughter, hugs and kisses, for being my co-gamer, and the mutual crazyness that drives your mom crazy.

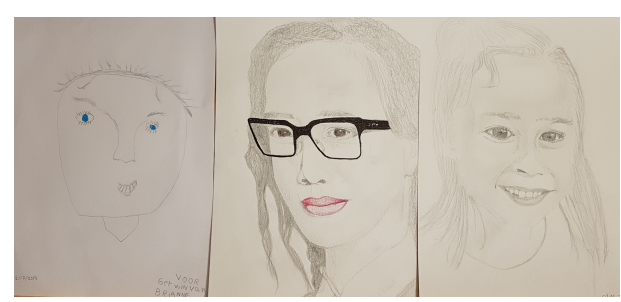

Gihigugma kaajo ko ikaw.

...One journey has ended, another is about to begin ...

- Gerwin Steen 16-01-2019, Leeuwarden, The Netherlands 
